\title{
Global Constraints as Graph Properties on Structured Network of Elementary Constraints of the Same Type
}

\author{
Nicolas Beldiceanu \\ SICS \\ Lägerhyddvägen 18 \\ SE-75237 Uppsala, Sweden \\ Email: nicolas@sics.se \\ January 282000 \\ SICS Technical Report T2000/01 \\ ISSN 1100-3154 \\ ISRN: SICS-T--2000/01-SE
}

\begin{abstract}
This report introduces a classification scheme for the global constraints. This classification is based on four basic ingredients from which one can generate almost all existing global constraints and come up with new interesting constraints. Global constraints are defined in a very concise way, in term of graph properties that have to hold, where the graph is a structured network of same elementary constraints. Since this classification is based on the internal structure of the global constraints it is also a strong hint for the pruning algorithms of the global constraints.
\end{abstract}

Keywords Constraint, finite domain, global constraint, classification, resource constraint scheduling, graph partitioning, timetabling. 


\section{Table of contents}

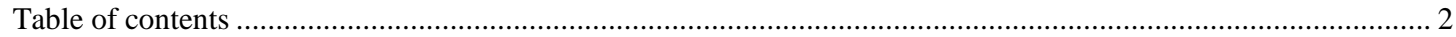

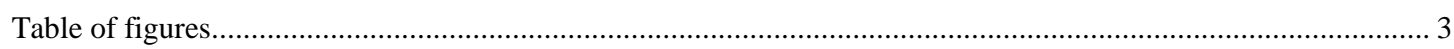

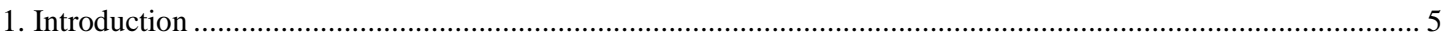

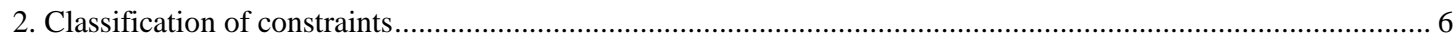

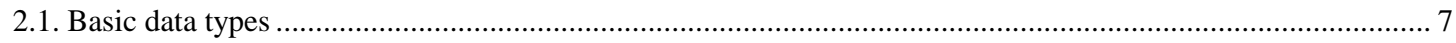

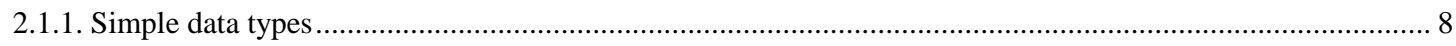

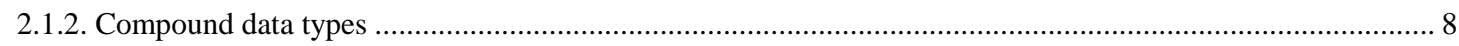

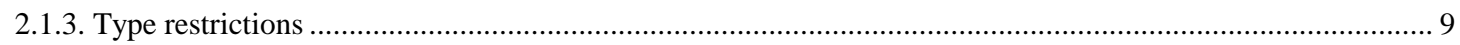

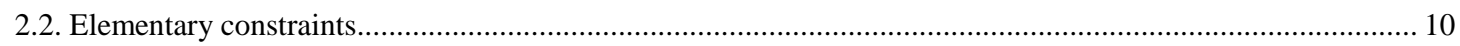

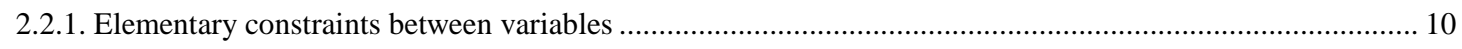

2.2.2. Elementary constraints between multi-dimensional rectangles.............................................................. 11

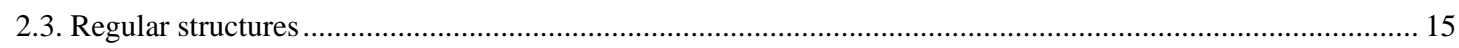

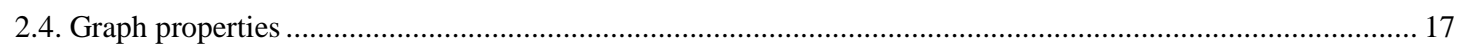

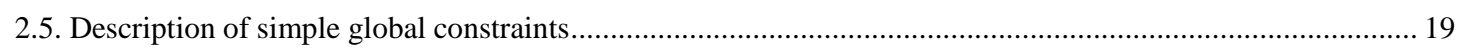

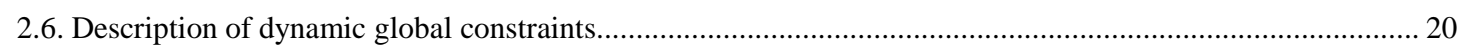

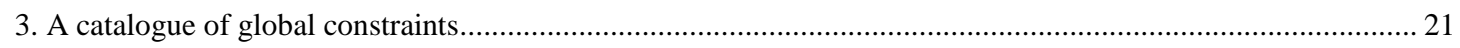

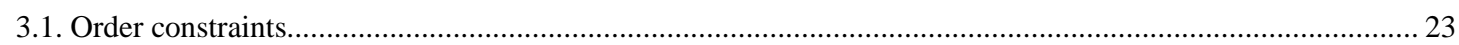

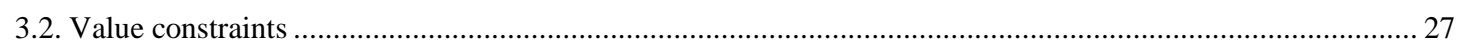

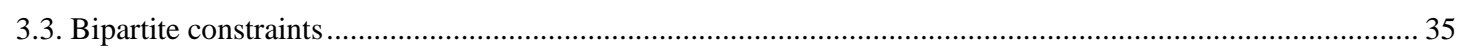

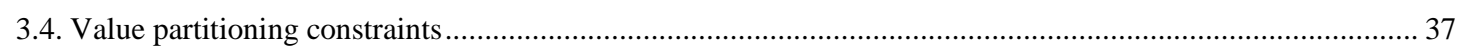

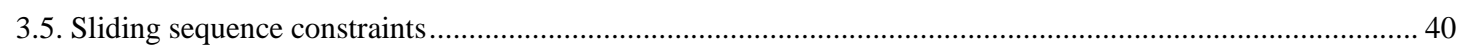

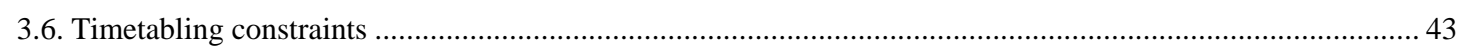

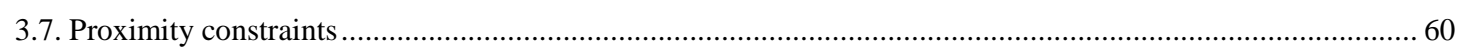

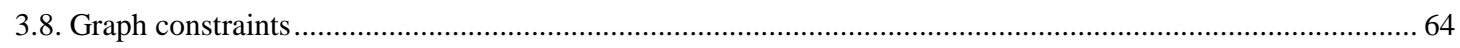

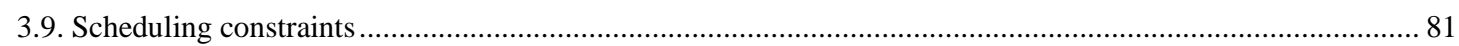

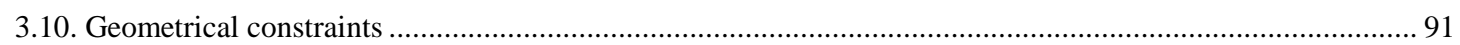

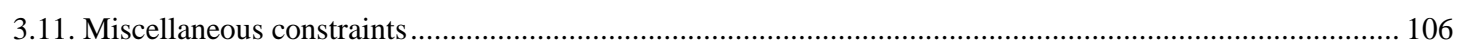

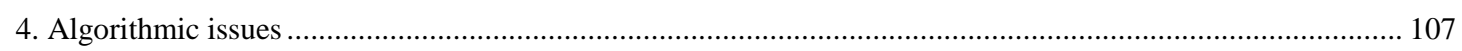

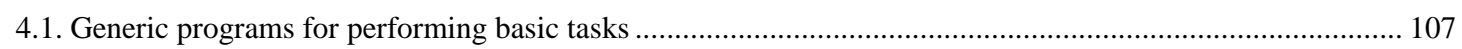

4.2. Searching for general relations that links different graph characteristics.................................................... 107

4.3. Creation of propagation algorithms specific to a given family of global constraints .................................... 108

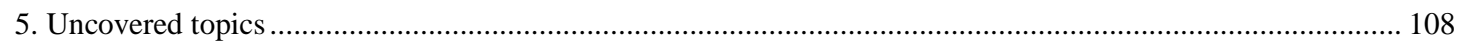

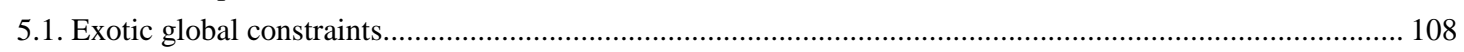

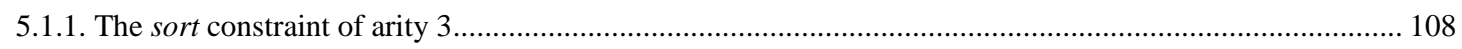

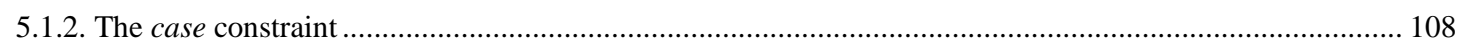

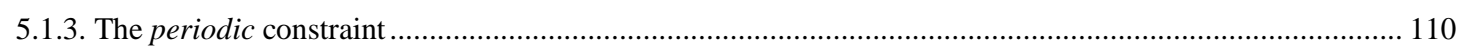

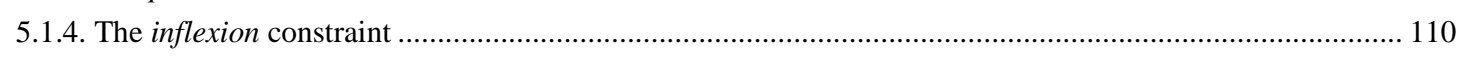

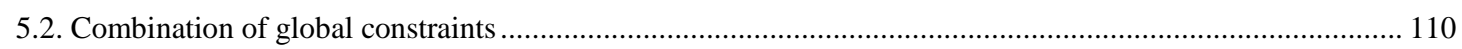

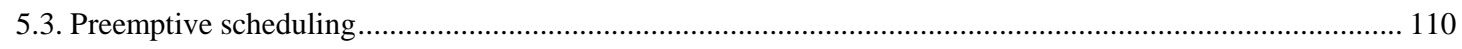

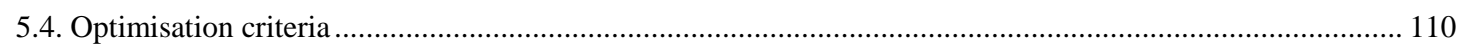

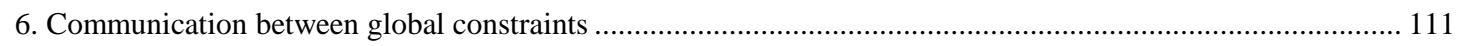

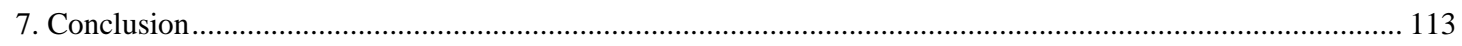

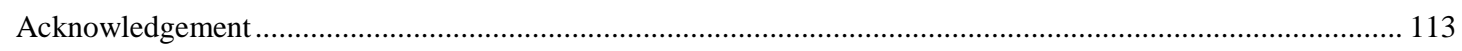

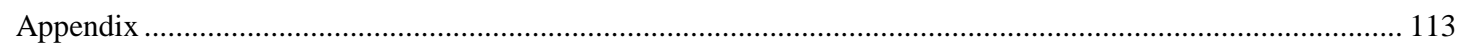

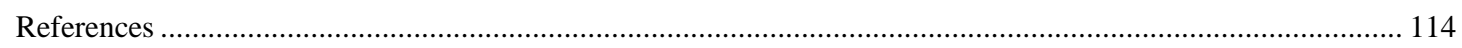

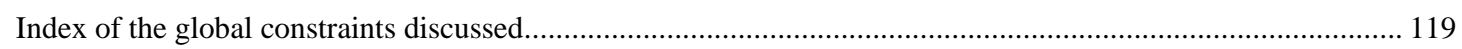




\section{Table of figures}

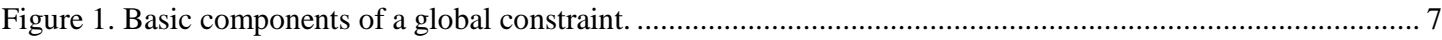

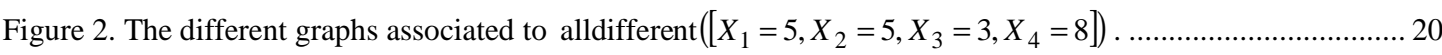

Figure 3. Initial and primal graphs associated to the cumulative instance ....................................................... 21

Figure 4. The different dimensions of the global constraint "space". .............................................................. 21

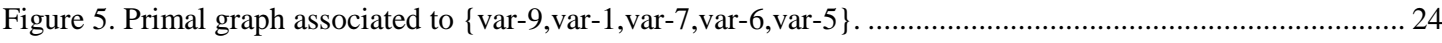

Figure 6. Primal graph associated to the $\min _{-} n$ constraint and corresponding ranks......................................... 25

Figure 7. Primal graph associated to the $m a x \_n$ constraint and corresponding ranks......................................... 26

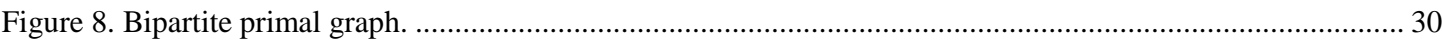

Figure 9. The 4 connected components of the bipartite primal graph.............................................................. 31

Figure 10. The 3 connected components of the bipartite primal graph............................................................. 32

Figure 11. Assignment solution and primal graph with its 2 maximum cliques. .................................................. 33

Figure 12. An assignment with at most 2 distinct colors in parallel and the related primal graph with its maximum cliques.

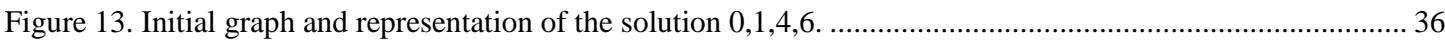

Figure 14. Final primal graph with one strongly connected component for each class of values. .......................... 39

Figure 15. Directed primal hypergraph associated to the example. ................................................................ 42

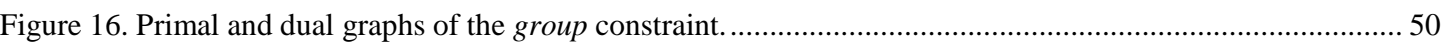

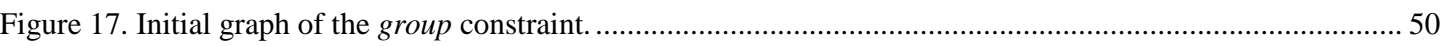

Figure 18. Primal and dual graphs associated to group_skip_isolated_item...................................................... 52

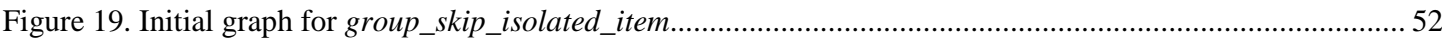

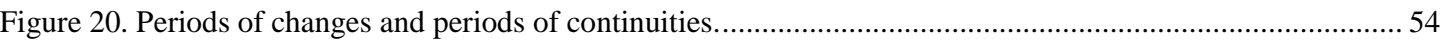

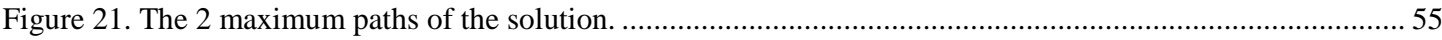

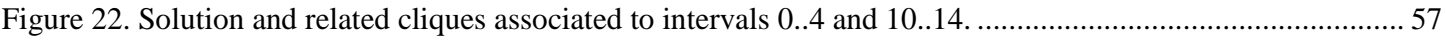

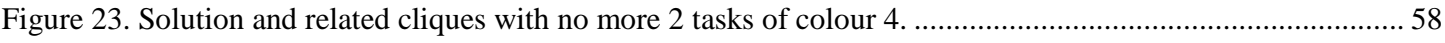

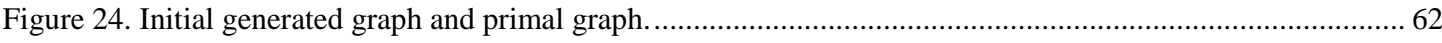

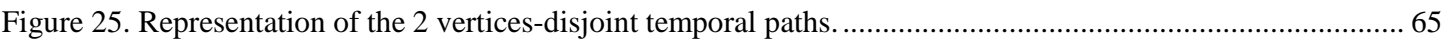

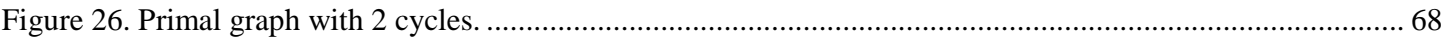

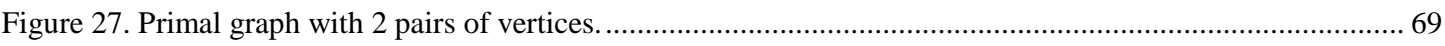

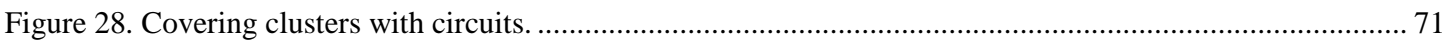

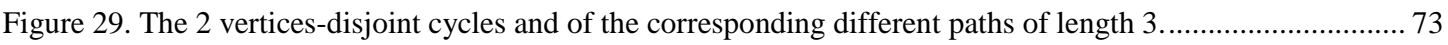

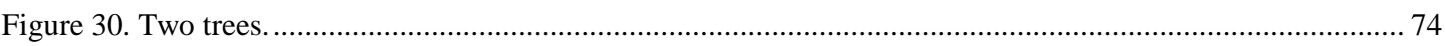

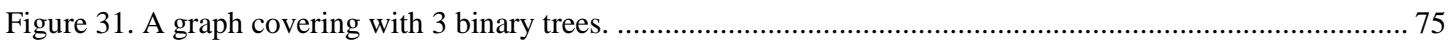

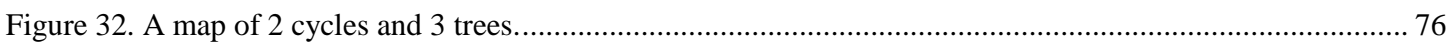

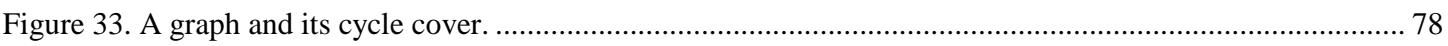

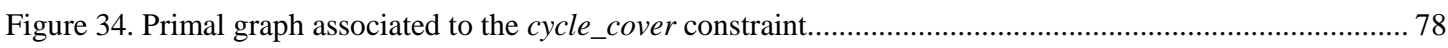

Figure 35. Primal graph associated to the facilities location problem................................................................ 79

Figure 36. Cumulative solution and primal graph with its 2 maximum cliques................................................... 81

Figure 37. A coloured cumulative solution with at most 2 distinct colours in parallel and the related primal graph

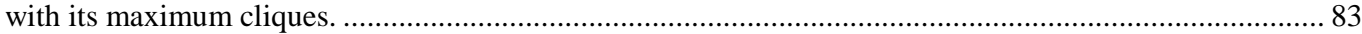

Figure 38. Cyclic cumulative solution and primal graph with its 2 maximum cliques. ....................................... 86

Figure 39. A cumulative with machine-assignment solution and the related primal graph with its 3 maximum cliques.

Figure 40. A coloured cumulative with machine-assignment solution and the related primal graph with its 3

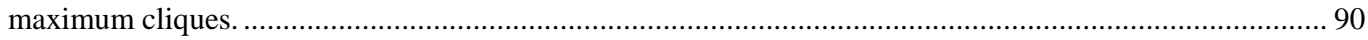

Figure 41. Bin-packing solution and related primal graph with its 2 maximum cliques....................................... 91

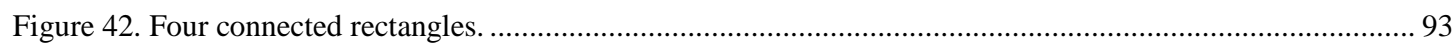

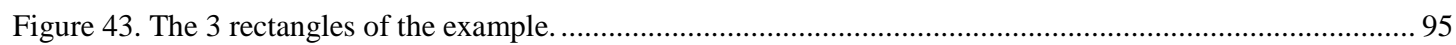

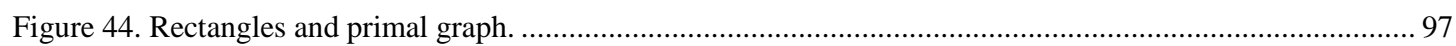

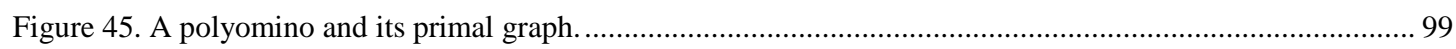

Figure 46. A graph covering with 2 line-segments intersections. ................................................................. 100

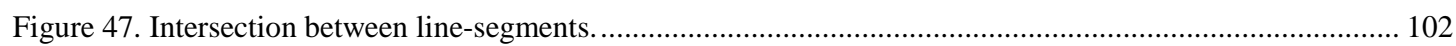




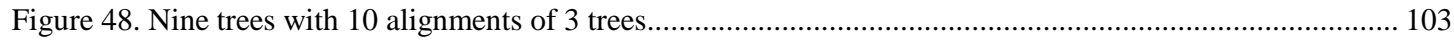

Figure 49. Two representations of a 2-dimensional cumulated profile........................................................... 105

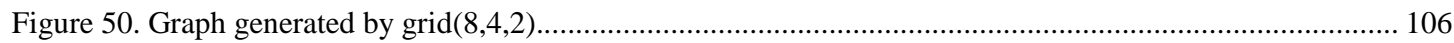

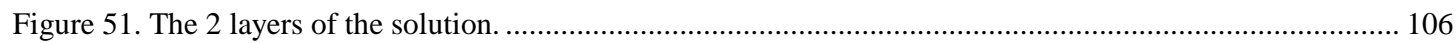

Figure 52. 2-dimensional element constraint and calendar constraint .......................................................... 109 


\section{Introduction}

The purpose of this report is to give new ideas in the finite domain constraint field. Nowadays, one can find constraints in an increasingly large number of areas such as constraint logic programming [VAN HENTENRYCK 89], concurrent constraint programming [SARASWAT 87], object [ROY, LIRET \& PACHET 99] and data base technologies [GAEDE, BRODSKY, GÜNTHER, SRIVASTAVA, VIANU \& WALLACE 97], meta programming [PITRAT 93, pages 163-187], concurrent programming language design [HARIDI, VAN ROY, BRAND \& SCHULTE 98], design of algorithms that combine different paradigms [CASEAU \& LABURTHE 96a], operation research [GENDREAU 99], linear programming [VAN HENTENRYCK 99] and AI [DECHTER \& PEARL 87]. As a matter of fact this is quite a good achievement for the constraint paradigm that has become popular so rapidly. However, this has also led to unexpected bad consequences:

- The first one is that there is no real common agreement on how constraints should be made available. This is perhaps the case because each framework carries its own advantages and drawbacks. Too much energy was spent on discussions about what is the best framework to use.

- A second bad consequence is that, because constraints are used is so many different fields, it is not easy for people with different skills to communicate and to understand each other. Too much time was devoted to implement the same constraint solver again and again in different frameworks and to rediscover the wheel [LAURIÈRE 96, page 2]. People from different origins addressed similar problems. This was for example the case for the complete pruning of the alldifferent constraint (i.e. the characterisation of the edges of all maximum matching of a bipartite graph) that was solved independently by Costa [COSTA 94] and Régin [RÉGIN 94].

- A third bad consequence is that, because so many people from different areas claim that using constraints within their preferred framework will be relevant for solving real problems, this undermines the credibility of constraint for people from discrete mathematics and algorithmic. This is perhaps because people from discrete mathematics and from algorithmic are used to carefully design algorithms that take benefit from the mathematical structure of the problem they are considering.

One of the main reasons why constraints have become so popular is that, since a few years, constraints were used in the industry in order to solve real problems [SIMONIS 96]. This process was initiated by the series of problems presented in [LAURIÈRE 76], [LAURIÈRE 78, pages 76-79]. In specific areas like scheduling, the key reason of success was because specialized abstractions [BELDICEANU \& CONTEJEAN 94], [RÉGIN 96], which use domain dependent algorithms were provided. However, one common reproach from a constraint perspective was that these abstractions were ad-hoc and that the trend of constraint programming was going more in direction of operations research than in the direction of the initial declarativeness of constraint.

One other problem that was pointed out in [BESSIÈRE 99, pages 25-26] concerns the fact that, from the pressure of real life problems, there were an increasing number of diverse global constraints. Peoples who tackle specific problems requested this, in order to avoid programming their own constraints on top of very basic mechanisms like demons, which ends up with poor propagation. However this again leads to an increasing number of ad-hoc global constraints, which make it very difficult to integrate in a homogeneous way global constraints with other tasks such as:

- Design generic visualisation interface for global constraints [SIMONIS, AGGOUN, BELDICEANU \& BOURREAU 00],

- Generate linear relaxations that are associated to the global constraints,

- Express heuristics that depend on some internal characteristic ${ }^{1}$ of the global constraints,

- Create a visual constraint language that integrates global constraints [SIMONIS 98].

Coming up with a generic description of global constraints is a necessary prerequisite if one wants to perform the previous tasks in a systematic way. This will be the main contribution of this report.

For all mentioned reasons, our main research goal will focus more on the constraints themselves and on the understanding of their internal constituents. For this purpose, we introduce a classification scheme of the global constraints and show how this classification can constitute a basis in order to come up with algorithms that are based on the structure of the constraints we consider.

After these preliminaries, we describe the content of the different sections of this report:

- The second section proposes a classification scheme for the "constraint jungle" we currently face. We do not consider any more global constraints as predefined abstractions; global constraints are now defined, in a very concise way, in term of graph properties that have to hold, where the graph corresponds to a structured network of same elementary constraints.

- The third section shows how this new classification scheme allows redefining most of all currently existing predefined global constraints. We describe a broad range of global constraints in various domains such as scheduling, placement, geometry, regulation and graph covering with different kind of patterns.

\footnotetext{
${ }^{1}$ As it was pointed out in [LAURIÈRE 78 page 62], most of the heuristics of ALICE where related to the internal graph associated to the constraints: "nearly all the contests are in association with the graphical representation".
} 
- The fourth section discusses shortly the algorithmic issues that one can derive from the previous classification. One key point is that, since our entire networks have a very regular structure and since the same elementary constraint is used over the whole network, the constraint algorithms can take advantage of this regularity. Algorithms on graphs have been used extensively in the operational research and the constraint community on a case-by-case basis. We show that using graph algorithms and properties of graphs turn out to be a direct natural consequence of our classification scheme.

- The fifth section discusses some uncovered issues that were not addressed by the previous framework. These issues are related to exotic global constraints, to combination of different global constraints and to optimisation criteria.

- Finally, the sixth section proposes a new mechanism that permits a tight cooperation between constraints and which leads to more global constraint propagation. This allows avoiding creating artificial global constraints that were just introduced because of the lack of communication between constraints. We revisit different classical constraint propagation algorithms and illustrate how they can integrate this new communication mechanism.

Since they are referenced in this report, the global constraints cumulative [AGGOUN \& BELDICEANU 93], among, diffn and cycle [BELDICEANU \& CONTEJEAN 94] are briefly described in the appendix.

\section{Classification of constraints}

Over the past few years a variety of useful constraints have gradually been introduced in the finite domain constraint field in order to tackle scheduling, timetabling and vehicle routing problems. Quite often it appears that similar constraints were introduced with different naming schemes ${ }^{2}$ and different ways of presenting their arguments, this within different frameworks such as logic programming or object oriented programming. Each new constraint was presented either as a generalisation of a previous constraint [RÉGIN 94], [RÉGIN 96], or as a completely new abstraction that was not related to any previously existing constraints [BELDICEANU \& CONTEJEAN 94]. An attempt to be independent from any framework was to come out with an easy to use modelling language [VAN HENTENRYCK 99] that allows stating the constraints and the search procedure of a problem. However even within this approach all the constraints were considered as arbitrary predefined built-in that are strongly dependent of the set of constraints that is provided by the underlying solver.

If one looks to the operation research community, classifications have already been established in different areas. For example, if we consider scheduling, an early classification was settled by [GRAHAM, LAWLER, LENSTRA \& RINNOOY KAN 79] and was updated by [HERROELEN, DEMEULEMEESTER \& DE REYCK 98]. One common feature of all these classifications is that they are problem oriented. It means that they try to capture the description a complete family of problems from a specific area with a general unambiguous classification scheme. For this purpose they use high-level domain dependent abstractions like non-renewable resource that are not derived from some kind of "first principle". This was motivated by the fact that persons wanted to characterize the complexity [GAREY \& JOHNSON 79] of the corresponding problems. However, from a constraint perspective one is more interested in classifying all the constraints that occur in different problems, rather than to classify the problems themselves. This is perhaps due to the fact that one hopes that the mathematical insight gained for one category of constraints will be relevant for all problems that mention this category of constraints. One other reason for trying to classify the constraints rather than the problems themselves is that the number of constraints should be in some sense "lower" than the number of problems.

One of our goals is to come up with a classification that is more related to the internal structure of the constraints rather than to specific problems areas. In the long term, one should be able to link the characteristics of a constraint to the algorithms that have to be used in order to perform test and pruning for that constraint.

In this section we describe a classification of the finite domain constraints. This classification is based on the following basic ingredients from which one can reconstruct almost all existing finite domain constraints and come up with new interesting global constraints.

- Basic data structures that are used in order to pass parameters to the constraints,

- Elementary constraints (mainly unary and binary constraints),

- Graphs with a regular structure (i.e. clique, grid, cycle, ...),

- Properties on graphs structures (i.e. strongly connected component, source, ...).

The next figure summarizes the different basic ingredients we use in order to describe global constraints.

\footnotetext{
${ }^{2}$ It appears that, even within the same solver, a constraint has different names according to the amount of pruning that is provided!
} 


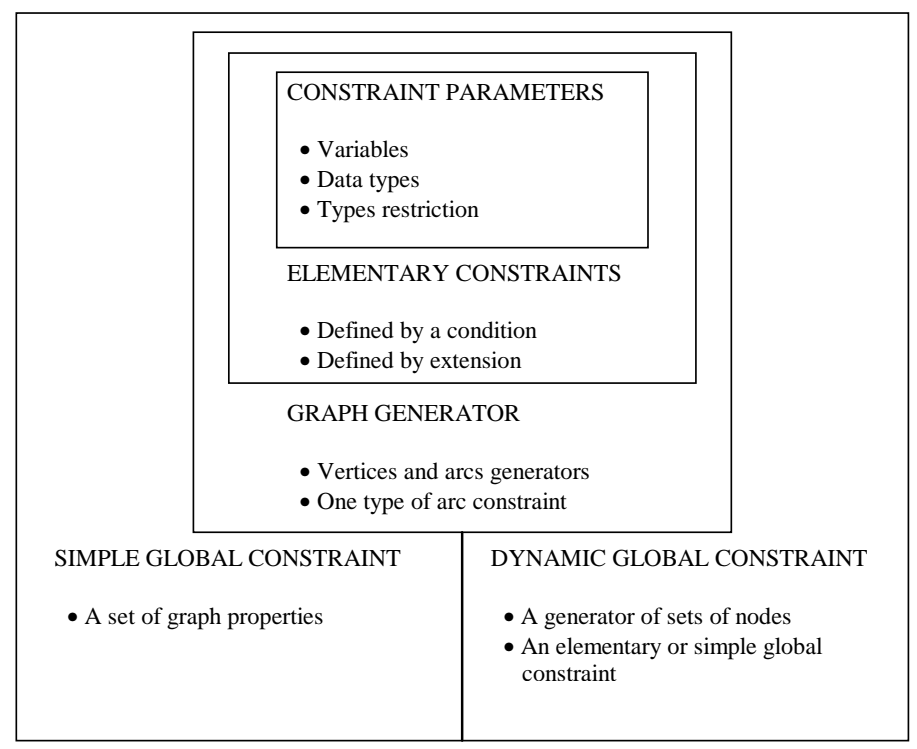

Figure 1. Basic components of a global constraint.

In the previous figure, we have introduce the following 2 types of global constraints:

- Simple global constraints are constraints for which one initially knows all variables that will be linked by a given condition.

- Dynamic global constraints are constraints for which one has to enforce a condition on different sets of variables that are not initially explicitly known.

We first start to explain each ingredient separately and then show how one can describe a global constraint in term of these basic ingredients.

\subsection{Basic data types}

Since constraints have to receive their arguments in some form, we first start to describe the abstract data types that we use in order to pass arguments to the constraints. When designing these basic data types we had these 4 main issues in mind.

- The data types are not related to any specific programming language like $\mathrm{C}, \mathrm{C}++$, Java or Prolog. If one wants to consider a specific language, then one has to map these abstract data types to the data types that are available within the considered programming language. This allows describing constraints in a way that is not related to any specific language. The goal is to come up with constraints that can be used on different platforms and frameworks.

- Most of the solvers that have evolved over a long period of time have to face the issue of migration from one language to an other language. This was the case for the following systems:

- CHIP [DINCBAS, VAN HENTENRYCK, SIMONIS, AGGOUN, GRAF \& BERTHIER 88] was moving from Prolog to $\mathrm{C}$ and from $\mathrm{C}$ to $\mathrm{C}++$,

- CLAIRE [CASEAU \& LABURTHE 96a] initially written in C++ is being currently rewritten in JAVA,

- Ilog Solver [ILOG 99] was switching from LeLisp to C++.

A similar migration problem was also pointed out in the final evaluation report of the ALCOM-IT [ALCOM 97] project for the LEDA [MEHLHORN \& NÄHER 99] library. In this case there was the question to move from $\mathrm{C}++$ to JAVA. Providing explicitly [PITRAT 93] the maximum amount of information about the constraints in a declarative form facilitates the migration from one language to one other language.

- Usually, the description of the parameters of a constraint is done in 2 phases. The first phase describes the syntax, while the second phase gives in some informal way additional conditions that have to hold on the parameters of the constraint. This way of proceeding has the main drawback that there is some information that is only accessible to humans and not to programs. This lead to the bad consequence that one can not design generic programs that take the description of a constraint in order to perform the following tasks:

- Create a parser for the constraint,

- Generate automatically tests cases to capture inconsistent pruning,

- Generate automatically test to capture missing propagations,

- Generate documentation.

One other reason to define explicitly the additional restrictions is that these conditions will sometime allow finding out properties of the constraint (see footnote of cycle constraint, page 67). For all these reasons, we will provide explicitly together with the data types, all additional conditions that have to hold. 
- Most of the finite domain constraints systems propose a set of constraints that express relations between variables. However some researcher have criticized this point [ROY, LIRET \& PACHET 99] and argue that in many applications one would prefer to state directly constraints between higher-level constructions such as objects. For example, if we consider a scheduling problem, we would naturally want to express constraints between tasks. Each task would have a set of attributes like its start, duration and end. For this reason we will introduce a data structure that corresponds to a collection of items. We use it extensively in order to pass parameters to the constraints.

\subsubsection{Simple data types}

We provide the following 3 basic data types "int", "atom" and "dvar" that respectively corresponds to an integer, to an atom and to a domain variable. Examples of predefined atoms are MININT and MAXINT that respectively correspond to the smallest and to the largest integer. All domain variables that will be used within the constraints are expression of the form $C \cdot X+D^{3}$ where $X$ is a variable that ranges over a finite set of integers and $C$ and $D$ are integers such that $C$ is different from zero. This is required if one wants to avoid to create extra variables or specific constraints that integrate $C$ and $D$ as ad-hoc parameters. We justify this choice by 2 examples where this is quite useful.

\section{EXAMPLE 1.}

The first example is related to the $n$-queen problem (i.e. place $n$ queens on a $n$ by $n$ chess board in such a way that no 2 queens are on the same row, the same column or the same diagonal). The most compact way to encode the $n$-queen problem is to use 3 alldifferent ${ }^{4}$ constraints of the following form:

- alldifferent $\left(\left[X_{1}, X_{2}, \ldots, X_{n}\right]\right)$,

- alldifferent $\left(\left[X_{1}, X_{2}+1, \ldots, X_{n}+n-1\right]\right)$,

- alldifferent $\left(\left[X_{1}+n-1, X_{2}+n-2, \ldots, X_{n}\right]\right)$.

From this example, one can observe that the fact to allow variables of the form $C \cdot X+D$ avoid the creation of extra variables.

\section{EXAMPLE 2}

The second example is related to a resource-scheduling problem described in [CHEMLA, DIAZ,

KERLIRSIN \& MANCHON 94]. We have a set of $n$ tasks that are characterised by the following attributes:

- The origin $O_{i}$ of task $i$,

- The shift $S_{i}$ of task $i$ that corresponds to a fixed period of time after which, the task will effectively start to use the resource; the resource will then be used until the end of task $i$,

- The duration $D_{i}$ of task $i$,

- The amount of resource $R_{i}$ that is used by task $i$.

The origins of the tasks are domain variables, while the shift, the duration and the amount of resource are non-negative integers. For expressing the capacity constraint over the set of tasks we would like to use a cumulative 5 [AGGOUN \& BELDICEANU 93] constraint of the form: cumulative $\left(\left[O_{1}+S_{1}, \ldots, O_{n}+S_{n}\right],\left[D_{1}, \ldots, D_{n}\right],\left[R_{1}, \ldots, R_{n}\right]\right.$, Limit $)$, where Limit matches the highest peak of resource utilisation. While this is not possible in current versions of the cumulative constraint, one can notice that it would be useful for the same reason as the one mentioned before. One other reason is that one would prefer to save the cost of propagating holes between 2 variables of the equality constraint that one would have to create.

\subsubsection{Compound data types}

We provide the following compound data types:

$$
\begin{aligned}
& \text { - } \operatorname{list}(T): \\
& \text { - } \operatorname{term}\left(t, T_{1}, \ldots, T_{n}\right): \\
& \text { - collection }\left(c, A_{1}, \ldots, A_{n}\right) \text { : }
\end{aligned}
$$
a list of elements of type $T$. a term of arity $n$ with a functor $t$ and $n$ arguments of given types $T_{1}, \ldots, T_{n}$. a collection with name $c$ of ordered items, each item having a set of attributes $A_{1}, \ldots, A_{n}$; each attribute is described by one of the following expression $a-T$ or $a-T-d$ where $a$ is the name of the attribute, $T$ the type of the attribute and $d$ a default value for the attribute.

\footnotetext{
${ }^{3}$ From an implementation point of view, we should perhaps introduce these variables in the constraint engine as an extra layer on top of the traditional domain variables.

${ }^{4}$ The constraint alldifferent $\left(\left[X_{1}, X_{2}, \ldots, X_{n}\right]\right)$ holds if all variables $X_{1}, X_{2}, \ldots, X_{n}$ are pairwise different.

${ }^{5}$ The complete definition of the cumulative constraint is given in appendix 1.
} 

exclusive: one should be able to decide for any instantiated data structure its type.

We use the following notation to describe instantiated data structures of the previous types:

- A list of elements $e_{1}, \ldots, e_{n}$ is denoted $\left[e_{1}, \ldots, e_{n}\right]$,

- A term with functor $t$ and arguments $a_{1}, \ldots, a_{n}$ is denoted $t\left(a_{1}, \ldots, a_{n}\right)$,

- A collection of $n$ items, each item having $m$ attributes, is denoted $\left\{a_{1}-v_{11} \ldots a_{m}-v_{1 m}, \ldots, a_{1}-v_{n 1} \ldots a_{m}-v_{n m}\right\}$; each item is separated from the previous item by a comma.

Let us illustrates with 2 examples, the types one can create. The first example concerns the creation of the RECTANGLES type while the second concern the PARTITIONS type.

EXAMPLE 1: definition of the RECTANGLES type

In this example we define the type RECTANGLES that corresponds to a collection of rectangles, each rectangle being defined by its origin, size and end in the first and second dimensions. For this purpose we use the following declaration:

$$
\begin{array}{r}
\text { RECTANGLES: collection(oril-dvar, sizl-dvar-1, end1-dvar, } \\
\text { ori2-dvar, siz2-dvar-1, end2-dvar) }
\end{array}
$$

By the previous declaration we define a collection of items, each item having the following six attributes ori1, siz1, end1, ori2, siz2 and end2 which are domain variables. The attributes siz1 and siz2 have the default value 1 .

EXAMPLE 2: definition of the PARTITIONS type

In this example we define the type PARTITIONS that corresponds to a collection of set of values, each value occurring only once. For this purpose we use the following declaration:

$$
\begin{aligned}
& \text { PARTITIONS: collection (p-VALUES) } \\
& \text { VALUES : collection (val-int) }
\end{aligned}
$$

By the previous declaration we define a collection of items, each item having one single attribute $p$ that corresponds to a collection of items, each item having one single attribute val that is an integer value.

\subsubsection{Type restrictions}

In the previous examples we would like to express additional conditions that refine the definition of the types. For instance for the type RECTANGLES we would like to state that one is not obliged to provide all 3 attributes ori1, sizl and end1 but that 2 attributes are enough. For the PARTITIONS type we would like to express the fact that all values val are distinct. For this purpose, we provide declarations that allow specifying explicitly additional conditions that have to hold. Each restriction has one the following form.

RESTRICTION

RELOP

$O P$

EXPRESSION

TERMINAL

DESIGNATOR

PATH $\rightarrow$ name RELOP EXPRESSION

name in $\{A T O M S\}$

distinct $(D E S I G N A T O R)$

ATTR_RESTRICTION

$\rightarrow \quad=|\neq| \leq|>| \geq \mid<$

$\rightarrow+|-|$.

$\rightarrow$ EXPRESSION RELOP EXPRESSION

EXPRESSION OP EXPRESSION

- EXPRESSION

$\min ($ EXPRESSION, EXPRESSION $)$

$\max ($ EXPRESSION, EXPRESSION)

TERMINAL

$\rightarrow$ int

|DESIGNATOR |

$\rightarrow$ name

name $P A T H$

$\rightarrow \quad /$ LINK

/ LINK PATH 


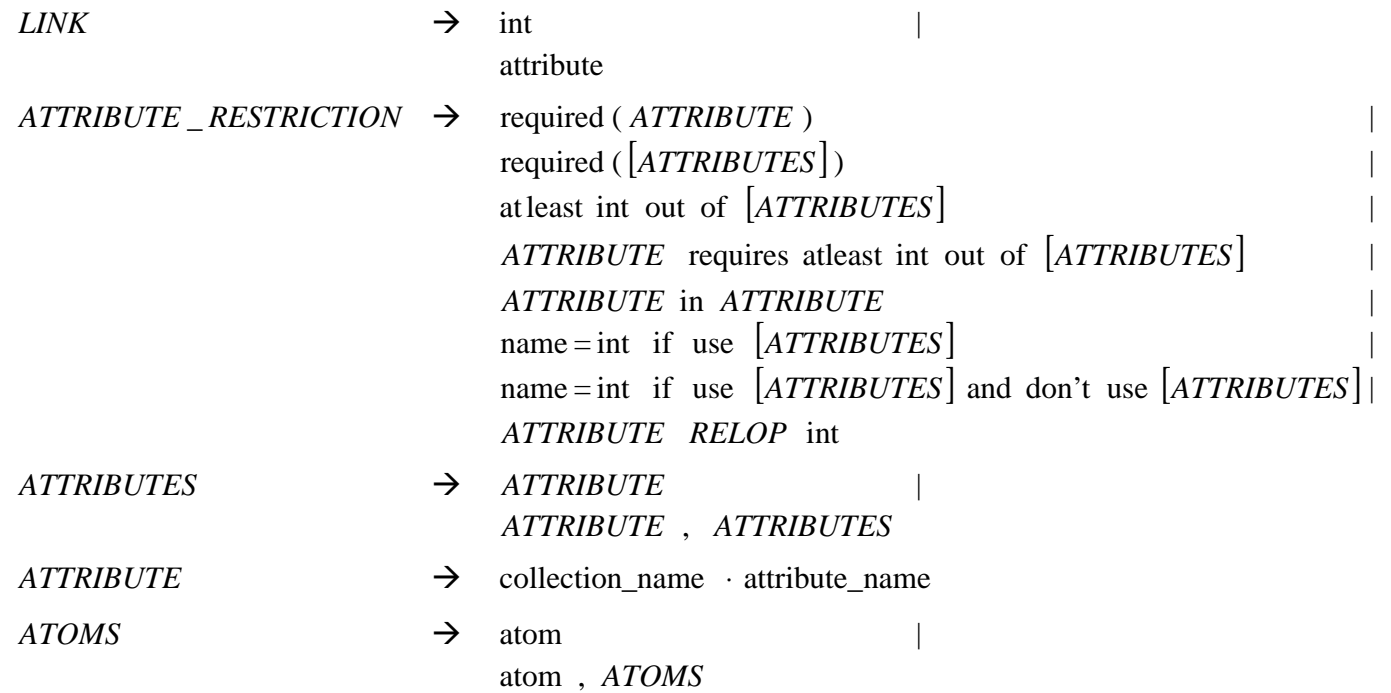

ATTRIBUTES

ATTRIBUTE

ATOMS

We consider again the 2 previous examples where we define the RECTANGLES and PARTITIONS types and we provide the additional restrictions that we want to hold.

EXAMPLE 1: definition of the RECTANGLES type

We complete the definition of the RECTANGLES type by providing several restrictions.

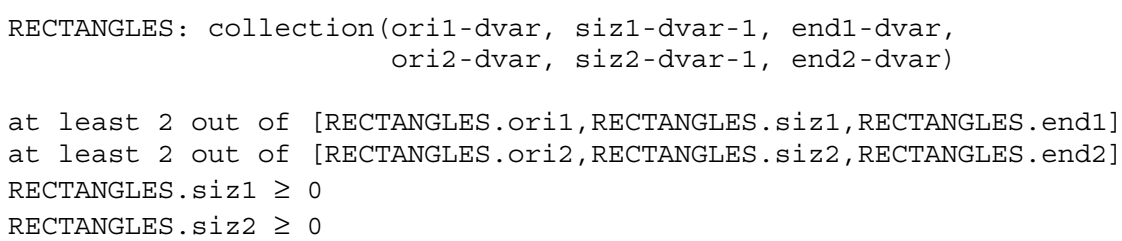

The first restriction states that we require at least 2 attributes out of the attributes ori1,sizl,end1 of the first dimension, while the second restriction imposes the same condition for the attributes of the second dimension. Finally, the 2 last conditions state that the size of the rectangle in the first and second dimension should both be non-negative. Note that the fact, that for each dimension the end is equal to the origin plus the size, is not considered as a restriction. It will be defined latter as a constraint.

EXAMPLE 2: definition of the PARTITIONS type

We complete the definition of the PARTITIONS type by providing 2 restrictions.

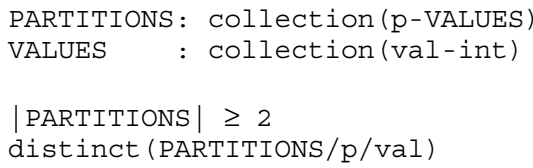

The first restriction express the fact that we should have at least 2 sets of values, while the second restriction imposes all values to be pairwise distinct.

\subsection{Elementary constraints}

This paragraph describes almost all elementary constraint that will be uses later. We first present the elementary constraints that link variables. In the second part, we introduce elementary constraints between $n$-dimensional rectangles.

\subsubsection{Elementary constraints between variables}

Each elementary constraint is described in the next table by using the following entries:

- Name: provides a symbolic name for the constraint,

- Arity: indicates the arity of the constraint; we use the letter "n" to show that a constraint can be used on any arity,

- Arguments: describes the type of each argument and the necessary restrictions,

- Parameters: describes the type of each parameter (i.e. fixed values) and the necessary restrictions,

- Purpose: explain what is the purpose of the constraint. 


\begin{tabular}{|c|c|c|c|c|}
\hline NAME & ARITY & ARGUMENTS & PARAMETERS & PURPOSE \\
\hline $\mathrm{Eq}$ & 1 & $\mathrm{X}:$ dvar & VAL: int & $\mathrm{X}=\mathrm{VAL}$ \\
\hline In & 1 & $\mathrm{X}:$ dvar & $\begin{array}{l}\text { VALUES : collection (val-int) } \\
\text { distinct (VALUES/val) }\end{array}$ & $\mathrm{X}$ in VALUES \\
\hline Equal & 2 & $\mathrm{X}, \mathrm{Y}: \mathrm{dvar}$ & & $X=Y$ \\
\hline Different & 2 & $\mathrm{X}, \mathrm{Y}: \mathrm{dvar}$ & & $X \neq Y$ \\
\hline Less & 2 & $\mathrm{X}, \mathrm{Y}: \mathrm{dvar}$ & & $X<Y$ \\
\hline Greater & 2 & $\mathrm{X}, \mathrm{Y}:$ dvar & & $\mathrm{X}>\mathrm{Y}$ \\
\hline GreaterEqual & 2 & $\mathrm{X}, \mathrm{Y}: \operatorname{dvar}$ & & $X \geq Y$ \\
\hline LessEqual & 2 & $\mathrm{X}, \mathrm{Y}:$ dvar & & $\mathrm{X} \leq \mathrm{Y}$ \\
\hline EqualInValues & 2 & $\mathrm{X}, \mathrm{Y}: \mathrm{dvar}$ & $\begin{array}{l}\text { VALUES : collection (val-int) } \\
\text { distinct (VALUES/val) }\end{array}$ & $(\mathrm{X}$ in VALUES $) \wedge(\mathrm{X}=\mathrm{Y})$ \\
\hline EqualoutJoker & 2 & $\mathrm{X}, \mathrm{Y}:$ dvar & JOKER : int & $\mathrm{X} \neq \mathrm{JOKER} \wedge \mathrm{X}=\mathrm{Y}$ \\
\hline LessMod & 2 & $\mathrm{X}, \mathrm{Y}: \mathrm{dvar}$ & $\begin{array}{l}M: \text { int } \\
M>0\end{array}$ & $(\mathrm{X} \bmod \mathrm{M})<(\mathrm{Y} \bmod \mathrm{M})$ \\
\hline DiffIncMod & 2 & $\mathrm{X}, \mathrm{Y}: \mathrm{dvar}$ & $\begin{array}{l}M: \text { int } \\
M>0\end{array}$ & $(\mathrm{X}+1) \bmod \mathrm{M}) \neq \mathrm{Y}$ \\
\hline DiffIncModM & 2 & $\mathrm{X}, \mathrm{Y}: \operatorname{dvar}$ & $\begin{array}{l}\mathrm{M}: \text { int } \\
\mathrm{M}>0\end{array}$ & $\begin{array}{l}((\mathrm{X}+1) \bmod \mathrm{M}) \neq \mathrm{Y} \wedge \\
\mathrm{X}<\mathrm{M} \wedge \mathrm{Y}<\mathrm{M}\end{array}$ \\
\hline EqualMod & 2 & $\mathrm{X}, \mathrm{Y}:$ dvar & $\begin{array}{l}M: \text { int } \\
M>0\end{array}$ & $\mathrm{X} \equiv \mathrm{Y} \quad(\bmod \mathrm{M})$ \\
\hline AbsGreater & 2 & $\mathrm{X}, \mathrm{Y}:$ dvar & $\begin{array}{l}\mathrm{D}: \text { int } \\
\mathrm{D} \geq 0\end{array}$ & $|X-Y|>D$ \\
\hline InSamePart & 2 & $\mathrm{X}, \mathrm{Y}:$ dvar & $\begin{array}{l}\text { PARTITIONS: collection (p-VALUES) } \\
\text { VALUES : collection (val-int) } \\
\text { |PARTITIONS } \mid \geq 2 \\
\text { distinct (PARTITIONS/p/val) }\end{array}$ & 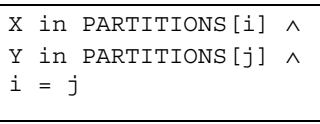 \\
\hline InDistPart & 2 & $\mathrm{X}, \mathrm{Y}:$ dvar & $\begin{array}{l}\text { PARTITIONS: collection (p-VALUES) } \\
\text { VALUES : collection (val-int) } \\
\text { |PARTITIONS | } \geq 2 \\
\text { distinct (PARTITIONS/p/val) }\end{array}$ & $\begin{array}{l}X \text { in PARTITIONS[i] } \wedge \\
Y \text { in PARTITIONS }[j] \wedge \\
i \neq j\end{array}$ \\
\hline SameGroup & 2 & $\mathrm{X}, \mathrm{Y}: \operatorname{dvar}$ & $\begin{array}{l}\text { VALUES : collection (val-int) } \\
\text { distinct (VALUES/val) }\end{array}$ & $\begin{array}{l}(\mathrm{X} \text { in VALUES }) \\
(\mathrm{Y} \text { in VALUES })\end{array}$ \\
\hline Sum & $\mathrm{n}$ & $\mathrm{Xi}$ :dvar & $\begin{array}{l}\text { INF }: \text { int } \\
\text { SUP }: \text { int } \\
\text { SUP } \geq \text { INF }\end{array}$ & $\mathrm{INF} \leq \Sigma \mathrm{Xi} \leq \mathrm{SUP}$ \\
\hline Card & $\mathrm{n}$ & $\mathrm{Xi}$ :dvar & $\begin{array}{l}\text { VALUES : collection (val-int) } \\
\text { INF : int } \\
\text { SUP }: \text { int } \\
\text { distinct (VALUES/val) } \\
\text { SUP } \geq \text { INF }\end{array}$ & 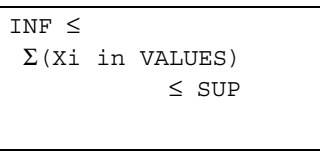 \\
\hline Range & $\mathrm{n}$ & Xi:dvar & $\begin{array}{l}\text { INF }: \text { int } \\
\text { SUP }: \text { int } \\
\text { SUP } \geq \text { INF }\end{array}$ & $\begin{array}{l}\text { INF } \leq \\
\quad \max (\mathrm{X} 1, \ldots, \mathrm{Xn})- \\
\quad \min (\mathrm{X} 1, \ldots, \mathrm{Xn}) \leq \mathrm{SUP}\end{array}$ \\
\hline SameDifference & 2 & \multicolumn{2}{|c|}{$\mathrm{X}, \mathrm{Y}$ :collection (p-dvar, $\mathrm{q}$-dvar) } & $X \cdot p-X \cdot q=Y \cdot p-Y \cdot q$ \\
\hline SucC & 2 & \multicolumn{2}{|c|}{$\begin{array}{l}\mathrm{X}, \mathrm{Y}: \text { item of NODES } \\
\text { NODES : collection (index-int, } \\
\text { Nucc-dvar) } \\
\text { NODES. index } \geq 1 \\
\text { NODES. index } \leq \mid \text { NODES } \mid \\
\text { distinct (NODES/index) } \\
\text { NODES. sucC } \geq 1 \\
\text { NODES. sucC } \leq \mid \text { NODES } \mid\end{array}$} & $\mathrm{X}$. succ $=\mathrm{Y}$. index \\
\hline Inverse & 2 & \multicolumn{2}{|c|}{ 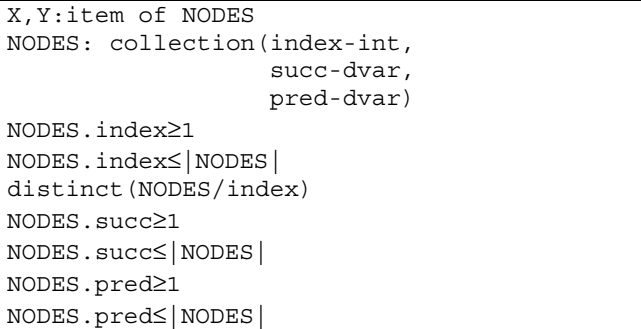 } & $\begin{aligned}(\mathrm{X} \cdot \text { succ } & =\mathrm{Y} \cdot \text { index } \wedge \\
\mathrm{Y} \cdot \text { pred } & =\mathrm{X} \cdot \text { index })\end{aligned}$ \\
\hline
\end{tabular}

Table 1. Elementary constraints between variables.

\subsubsection{Elementary constraints between multi-dimensional rectangles}

The purpose of this paragraph is to introduce a binary constraint between multi-dimensional rectangles that will be used later as a predefined binary constraint in structured constraint networks. Let Quad $_{i}$ be a rectangle in ndim dimensions defined by:

- An ordered set of ndim domain variables $O_{i, 1}, \ldots, O_{i, \text { ndim }}$, which corresponds to the origins of rectangle $\operatorname{Quad}_{i}$ in the different dimensions,

- An ordered set of ndim domain variables $S_{i, 1}, \ldots, S_{i, \text { ndim }}$, which corresponds to the sizes of rectangle $\operatorname{Quad}_{i}$ in the different dimensions, 
- An ordered set of ndim domain variables $E_{i, 1}, \ldots, E_{i, \text { ndim }}{ }^{6}$, which corresponds to the ends of rectangle $Q_{u a d}$ in the different dimensions,

such that the following conditions apply: $\forall d \in 1$..ndim : $E_{i d}-O_{i d}=S_{i d}$.

Let $D$ be a non-empty subset of 1. ndim and $C$ a vector of ndim strictly positive integers $C_{1}, . ., C_{\text {ndim }}$. For 2 rectangles $\operatorname{Quad}_{i}$ and $\mathrm{Quad}_{j}$, and a given set of dimensions $D$ we define the following functions:

- ori_siz_end $\left(\operatorname{Quad}_{i}, \operatorname{Quad}_{j}, D\right)=\sum_{d \in D}\left(\left(O_{i d}+S_{i d}=E_{i d}\right)+\left(O_{j d}+S_{j d}=E_{j d}\right)\right)$,

- noverlap $\left(\operatorname{Quad}_{i}, \operatorname{Quad}_{j}, D\right)=\sum_{d \in D} \min \left(1, \max \left(0, \min \left(E_{i d}, E_{j d}\right)-\max \left(O_{i d}, O_{j d}\right)\right)\right)$,

- cyclic_noverlap $\left(\operatorname{Quad}_{i}, \operatorname{Quad}_{j}, D, C\right)=\sum_{d \in D} \min \left(1, \max \left(0, \operatorname{coverlap}\left(O_{i d}, S_{i d}, E_{i d}, O_{j d}, S_{j d}, E_{j d}, C_{d}\right)\right)\right)$ where coverlap $\left(O_{1}, S_{1}, E_{1}, O_{2}, S_{2}, E_{2}, c\right)=\max \left(\operatorname{coverlap} 1\left(O_{1}, E_{1}, O_{2}, E_{2}, c\right), \operatorname{coverlap} 2\left(O_{1}, S_{1}, E_{1}, O_{2}, S_{2}, E_{2}, c\right)\right)$

coverlap1 $\left(O_{1}, E_{1}, O_{2}, E_{2}, c\right)=\left\{\begin{array}{l}\left(O_{1}<E_{1}\right) \cdot\left(O_{2}<E_{2}\right) \cdot\left(\min \left(E_{1}, E_{2}\right)-\max \left(O_{1}, O_{2}\right)\right)+ \\ \left(O_{1}<E_{1}\right) \cdot\left(O_{2} \geq E_{2}\right) \cdot\left(\max \left(0, E_{2}-O_{1}\right)+\max \left(0, E_{1}-O_{2}\right)\right)+ \\ \left(O_{1} \geq E_{1}\right) \cdot\left(O_{2}<E_{2}\right) \cdot\left(\max \left(0, E_{1}-O_{2}\right)+\max \left(0, E_{2}-O_{1}\right)\right)+ \\ \left(O_{1} \geq E_{1}\right) \cdot\left(O_{2} \geq E_{2}\right) \cdot\left(\min \left(E_{1}, E_{2}\right)-\max \left(O_{1}, O_{2}\right)+c\right),\end{array}\right.$

coverlap2 $\left(O_{1}, S_{1}, E_{1}, O_{2}, S_{2}, E_{2}, c\right)=\left\{\begin{array}{l}\min \left(S_{1}+S_{2}\right)- \\ \max \left(\left(E_{1}=0\right) \cdot c,\left(E_{1} \neq 0\right) \cdot E_{1},\left(E_{2}=0\right) \cdot c,\left(E_{2} \neq 0\right) \cdot E_{2}\right)+ \\ \min \left(O_{1}, O_{2}\right),\end{array}\right.$

- distance $\left(\operatorname{Quad}_{i}, \operatorname{Quad}_{j}, D\right)=\sum_{d \in D} \max \left(0, \min \left(E_{i d}, E_{j d}\right)-\max \left(O_{i d}, O_{j d}\right)\right)$,

• intersection $\left(\operatorname{Quad}_{i}, \operatorname{Quad}_{j}, D\right)=\left\{\begin{array}{l}\text { if } \exists d \in D / \min \left(E_{i d}, E_{j d}\right)>\max \left(O_{i d}, O_{j d}\right) \\ \text { then } \underset{d \in D / \min \left(E_{i d}, E_{j d}\right)>\max \left(O_{i d}, O_{j d}\right)}{\left.\prod_{j d}\right)-\max \left(O_{i d}, O_{j d}\right)} \\ \text { else } 0,\end{array}\right.$

•cyclic_intersection $\left(\right.$ Quad $_{i}$, Quad $\left._{j}, D, C\right)=\left\{\begin{array}{c}\text { if } \exists d \in D / \operatorname{coverlap}\left(O_{i d}, S_{i d}, E_{i d}, O_{j d}, S_{j d}, E_{j d}, C_{d}\right)>0 \\ \operatorname{then} \underset{d \in D / \operatorname{coverlap}\left(O_{i d}, S_{i d}, E_{i d}, O_{j d}, S_{j d}, E_{j d}, C_{d}\right)>0}{\left.\prod_{i d}, O_{j d}, S_{j d}, E_{j d}, C_{d}\right)} \\ \text { else } 0,\end{array}\right.$

- equal $\left(\operatorname{Quad}_{i}, \operatorname{Quad}_{j}, D\right)=\bigwedge_{d \in D}\left(\left(O_{i d}=O_{j d}\right) \wedge\left(E_{i d}=E_{j d}\right)\right)$,

- $\operatorname{ninclude}\left(\right.$ Quad $\left._{i}, \operatorname{Quad}_{j}, D\right)=\max \left(\sum_{d \in D}\left(O_{i d} \leq O_{j d} \leq E_{j d} \leq E_{i d}\right), \sum_{d \in D}\left(O_{j d} \leq O_{i d} \leq E_{i d} \leq E_{j d}\right)\right)$,

- before $\left(\operatorname{Quad}_{i}, \operatorname{Quad}_{j}, D\right)=\bigwedge_{d \in D}\left(E_{i d} \leq O_{j d}\right)$.

The previous quantities can be interpreted in the following way:

- ori_siz_end $\left(\operatorname{Quad}_{i}, \operatorname{Quad}_{j}, D\right)$ is the number of satisfied constraints that link the origin, the size and the end of both rectangles Quad $_{i}$ and $Q_{u a d}$ in the set of dimensions $D$.

- noverlap $\left(\operatorname{Quad}_{i}, \operatorname{Quad}_{j}, D\right)$ is the number of dimensions of the set $D$ where $\operatorname{Quad}_{i}$ and $\operatorname{Quad}_{j}$ overlap.

- cyclic_noverlap $\left(\operatorname{Quad}_{i}, \operatorname{Quad}_{j}, D, C\right)$ is the number of dimensions of the set D where $\operatorname{Quad}_{i}$ and $\operatorname{Quad}_{j}$ overlap according to the fact that we consider a cyclic placement space; the vector $C$ defines the size of the cyclic placement space in each dimension.

\footnotetext{
${ }^{6}$ If the size is not fixed and the end is more constrained than the origin then the end variable contains some information that you don't get when you consider only the origin and the size variables. Suppose that the origin, the size and the end vary between 1 and 5. If we don't give explicitly the end variable to the constraint, then the constraint would overestimate the latest end of the task to 10 (i.e. maximum of origin + maximum of size).
} 
- distance $\left(\operatorname{Quad}_{i}, \operatorname{Quad}_{j}, D\right)$ is the Manhattan distance between $\operatorname{Quad}_{i}$ and $\operatorname{Quad}_{j}$ according to the set of dimensions $D$.

- intersection $\left(\operatorname{Quad}_{i}, \operatorname{Quad}_{j}, D\right)$ is the product of the intersections of $\operatorname{Quad}_{i}$ and $\operatorname{Quad}_{j}$ in the dimensions of the set $D$ for which a non-empty intersection exists.

- cyclic_intersection $\left(\operatorname{Quad}_{i}, \mathrm{Quad}_{j}, D, C\right)$ is the product of the intersections, according to a cyclic placement space characterized by the vector $C$, of $Q_{u a d}$ and $Q_{u a d}$ in the dimensions of the set $D$ for which a nonempty intersection exists.

- equal $\left(\operatorname{Quad}_{i}, \operatorname{Quad}_{j}, D\right)$ holds if $\operatorname{Quad}_{i}$ and $\operatorname{Quad}_{j}$ coincide exactly in all dimensions of the set D.

- ninclude $\left(\operatorname{Quad}_{i}, \operatorname{Quad}_{j}, D\right)$ is the maximum value between the 2 following quantities: the number of dimensions of $D$ where $\operatorname{Quad}_{i}$ is included in $\operatorname{Quad}_{j}$, or the number of dimensions of $D$ where $\operatorname{Quad}_{j}$ is included in $\mathrm{Quad}_{i}$.

- before $\left(\operatorname{Quad}_{i}, \operatorname{Quad}_{j}, D\right)$ holds if for all dimensions of D, $\operatorname{Quad}_{i}$ ends not after $\operatorname{Quad}_{j}$ starts.

A simple binary constraint between multi-dimensional rectangles is one of the following expressions:

- $\wedge$ FUNCTION $(Q 1, Q 2, D)$ RELOP VALUES,

- V FUNCTION $(Q 1, Q 2, D)$ RELOP VALUES ,

where $Q_{1}$ and $Q_{2}$ refer respectively to $\operatorname{Quad}_{i}$ or $\operatorname{Quad}_{j}, D$ to a subset of 1..ndim, RELOP to the comparisons $=, \neq,<, \geq,>, \leq$, VALUES to a set of integers, and FUNCTION to the previous predefined functions.

A conditional binary constraint between multi-dimensional rectangles is the following expression:

- if $Q 1=Q 2$ then SIMPLE else SIMPLE,

where SIMPLE refers to a simple binary constraint.

The next table gives different examples of possible elementary constraints that will be used in this report. We first explicit the different types that are used within the table:

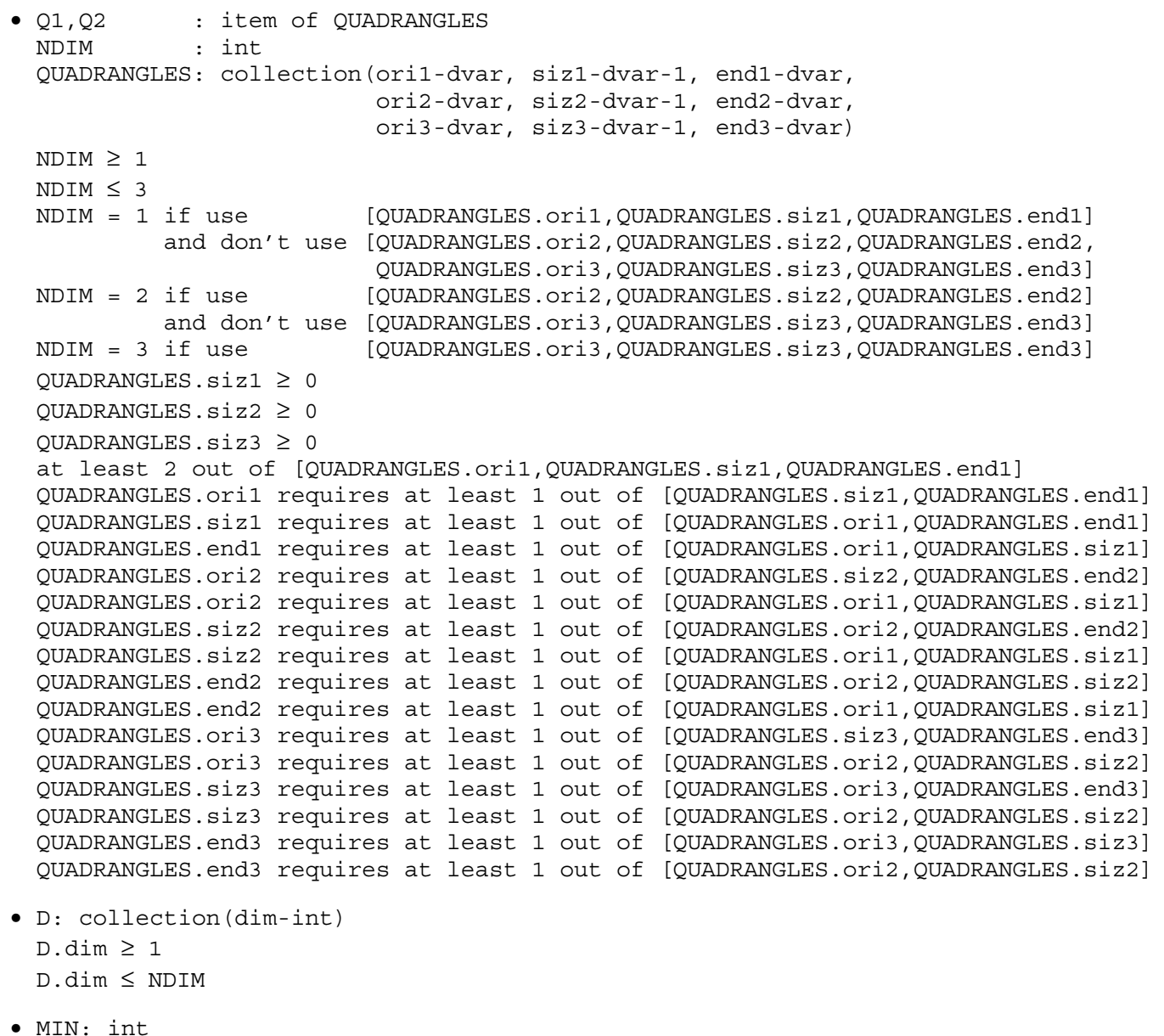


- MAX : int

- $d$ : int

$d \geq 1$

$d \leq$ NDIM

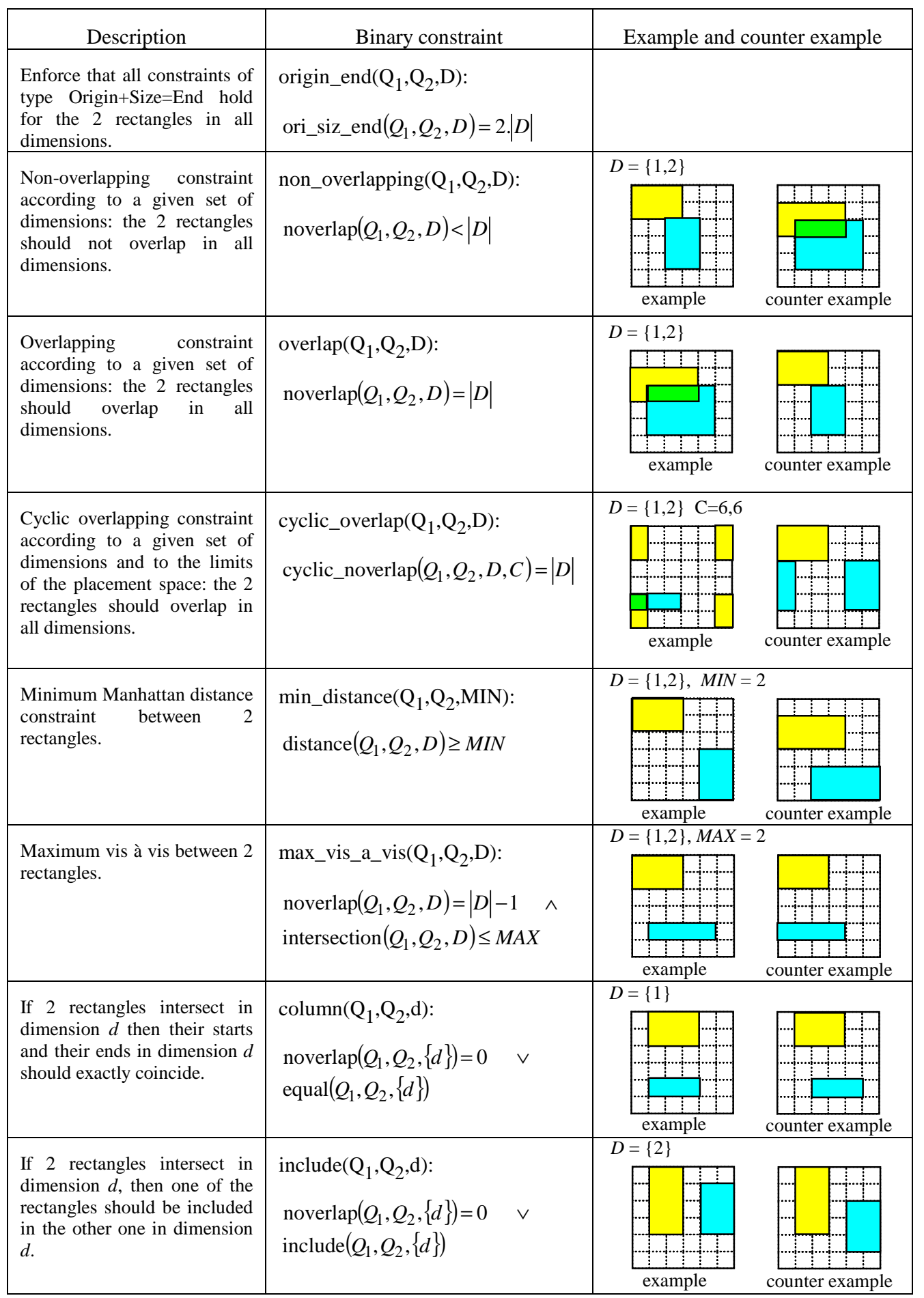




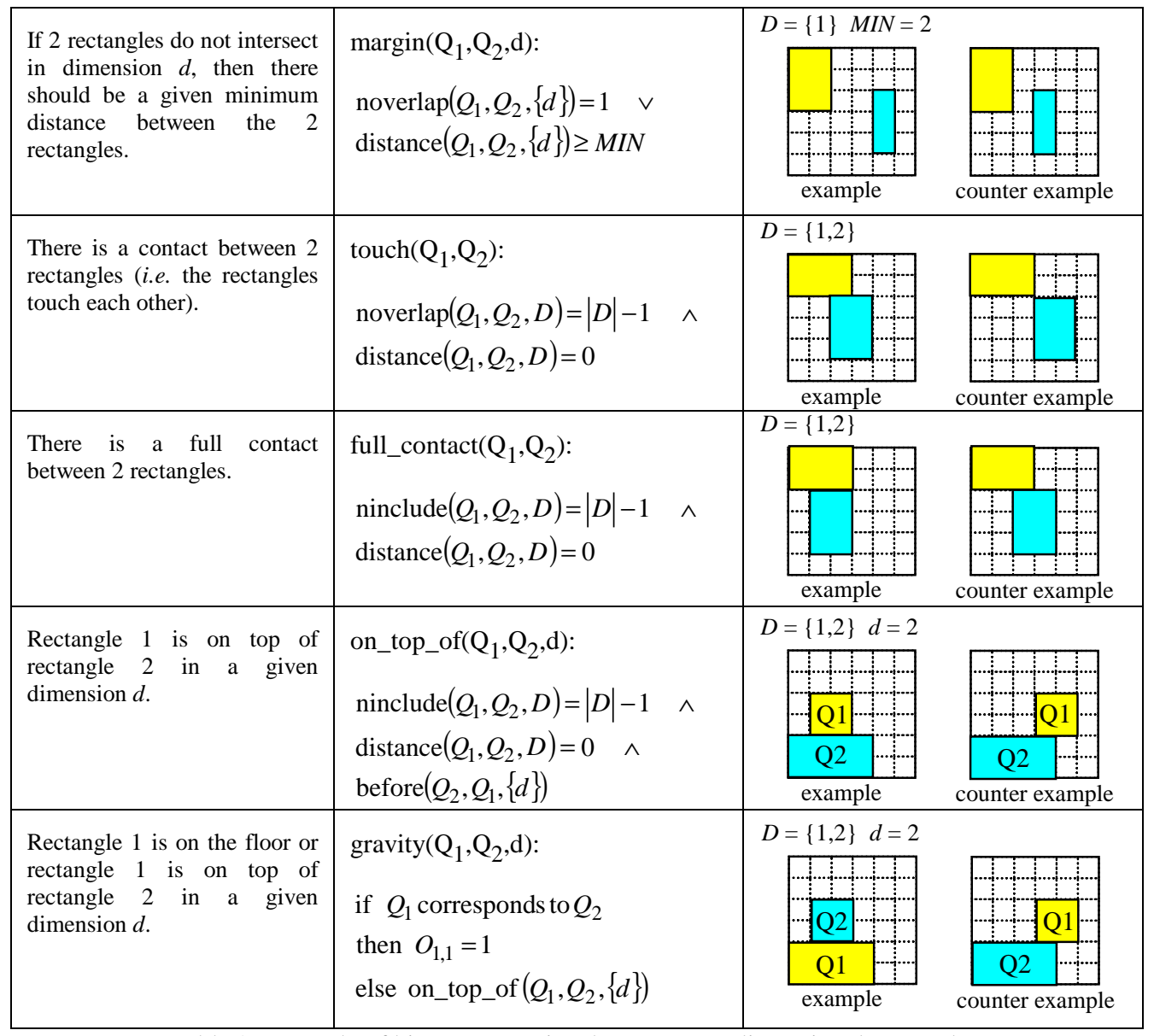

Table 2. Example of binary constraints between $n$-dimensional rectangles.

\subsection{Regular structures}

The purpose of this paragraph is to describe the third basic ingredient that we use in order to define global constraints. This ingredient corresponds to directed hypergraphs [BERGE 87], which have a very regular structure. They are defined in the following way. We consider $c$ collections of items. Each collection denoted $C_{i}(1 \leq i \leq c)$ has the same type of item and each attribute of an item is an integer or a domain variable.

- The vertices of the directed hypergraph are generated by a vertex generator, which takes as input the different collections of items $C_{i}(1 \leq i \leq c)$ and produces one or several collections of items, such that each item corresponds to one vertex of the directed hypergraph. The different available vertex generators will be described below.

- To all arcs of the directed hypergraph correspond the same $n$-ary constraint that involves $n$ vertices in a given order $^{7}$. These constraints, which are mainly unary and binary constraints, were described in the previous section. Since we are mostly ${ }^{8}$ interested in regular structures, we describe all the arcs with a set of predefined arc generators that correspond to classical regular structures one can find in the graph literature [SKIENA 90, pages 140-153]. An arc generator takes the different collections of items produced by the vertex generator, a given arity $n$, and returns the corresponding directed hypergraph.

The current available vertex generators are:

- The IDENTITY vertex generator takes one or several collections of items that have the same type and returns the same collections of items.

\footnotetext{
${ }^{7}$ Usually the edges of an hypergraph are not oriented [BERGE 87, pages 1-2]. However for our purpose we need to define an order on the vertices of an edge since the corresponding $n$ - ary constraint takes their arguments in a given order.

${ }^{8}$ However, for specific cases like graph colouring we leave open the possibility to define explicitly the graph. One can note that even in this case, the same family of constraints applies for all links of the graph: the disequality constraint which enforces to assign distinct colours to adjacent vertices.
} 
- The PAIR vertex generator takes one collection of items $C$ where each item has $n$ attributes, and returns a collection $C$ ' where each item has $2 \cdot n$ attributes. The items of collection $C^{\prime}$ are computed in the following way:

Let $|C|$ be the number of items of collection $C$.

$\forall i \in 1 .|C|, \forall j \in 1 . .|C|:$

- let $\left\{a_{1}-v_{i, 1} \ldots a_{n}-v_{i, n}\right\}$ be the $i$ th item of collection $C$,

- let $\left\{a_{1}-v_{j, 1} \ldots a_{n}-v_{j, n}\right\}$ be the $j$ th item of collection $C$.

Then $\left\{a_{1}-v_{i, 1} \ldots a_{n}-v_{i, n}, a_{1}-v_{j, 1} \ldots a_{n}-v_{j, n}\right\}$ is the $(i-1) \cdot|C|+j$ th item of collection $C^{\prime}$.

It is also possible to use a variant denoted PAIR (COMPARE) where COMPARE is one of the following comparison test: $=, \neq, \leq,>, \geq,<$. In this case, we only consider the pairs $i, j$ such that the condition $i$ COMPARE $j$ holds.

We now describe the arc generators. The next table gives examples of regular structures that we provide, where we apply each arc generator to 2 collections of items that respectively correspond to items $\{1,2\}$ and to items $\{3,4\}$. We use the following pictogram for the graphical representation of a constraint network. We employ a line for arity 1 , an arrow for arity 2, and an ellipse in the other cases. In these last cases, since the vertices of an arc are ordered, a circle at one of the extremity of the ellipse indicates the "direction" of the ellipse. For example, the ellipse that contains vertices 1, 2 and 3 means that a 3 -ary constraint is applied on items 1,2, and 3 in this specific order. For the grid generator we indicate the size of the grid in each dimension. For example, in the case of 2 dimensions, we indicate the number of vertices in each line and each column.

\begin{tabular}{|c|c|c|}
\hline ARC GENERATOR & ARC ARITY & EXAMPLE \\
\hline SELF & 1 & $(3)$ \\
\hline LOOP & 2 & $\frac{7}{(1)} \sqrt{\frac{1}{3}} \frac{\sqrt{4}}{4}$ \\
\hline PATH & 2 & (1) $\rightarrow$ (2) $\rightarrow$ (3) $\rightarrow$ (4) \\
\hline PATH & 3 & (1) (2) (3) (4). \\
\hline CIRCUIT & 2 & (1) $\rightarrow$ (2) $\rightarrow$ (3) $\rightarrow$ (4) \\
\hline CHAIN & 2 & (1) $\leftrightarrow$ (2) $\leftrightarrow(3) \leftrightarrow(4)$ \\
\hline CYCLE & 2 & (1) $\leftrightarrow$ (2) $\leftrightarrow$ (3) $\leftrightarrow(4)$ \\
\hline $\operatorname{GRID}([2,2])$ & 2 & \\
\hline CLIQUE & 2 & \\
\hline CLIQUE $(\neq)$ & 2 & (2) \\
\hline CLIQUE (<) & 2 & \\
\hline
\end{tabular}




\begin{tabular}{|l|l|l|}
\hline PRODUCT & 2 & \\
\hline PRODUCT (=) & 2 & \\
\hline LOOP PATH & 2 & $(2)$ \\
\hline
\end{tabular}

Table 3. Examples of regular structures produced by the arc generator.

Finally we mention that one can use more than one arc generator in order to generate the arcs of the directed hypergraph. This is actually the case for the last row of the previous table. The only restriction is that all arcs should have the same arity. We now give 2 concrete examples of directed hypergraphs that one would have to create in order to describe some specific constraint.

- The first constraint is about a placement problem where one wants to impose that different rectangles do not overlap each other ${ }^{9}$. The corresponding constraint takes as argument a collection of items that is defined in the following way:

RECTANGLES: collection(ori1-dvar, siz1-dvar, end1-dvar, ori2-dvar, siz2-dvar, end2-dvar) .

We use the arc generator CLIQUE to generate a binary constraint OVERLAP between each pairs of rectangles. Note that CLIQUE will also generate an arc between a vertex and itself.

- The second constraint is about a counting problem where one wants to count how many variables of a given set of variables take their value in a second set of variables ${ }^{10}$. In this case we have 2 collections of items that are both defined in the following way:

VARIABLES: collection (var-dvar).

We use the arc generator PRODUCT to generate a binary constraint Equal between each pair $\left(V A R_{1}, V A R_{2}\right)$ of variables where the first variable $V A R_{1}$ belongs to the first collection and the second variable $V A R_{2}$ to the second collection.

\subsection{Graph properties}

The purpose of this paragraph is to describe the last basic ingredient that we use in order to define global constraints. This ingredient corresponds to graph characteristics [BERGE 70], [GONDRAN \& MINOUX 79] which we will define now. Lets us first introduce some basic vocabulary on graph.

An oriented graph $G$ is a finite set $V(G)$ of vertices together with a set $E(G)$ of ordered pairs of vertices called directed edges or arcs.

We say that $e_{2}$ is a successor of $e_{1}$ if there exist an arc that starts from $e_{1}$ and ends at $e_{2}$. In the same way, we say that $e_{2}$ is a predecessor of $e_{1}$ if there exist an arc that starts from $e_{2}$ and ends at $e_{1}$.

A vertex of $G$ that does not have any predecessor is called a source. A vertex of $G$ that does not have any successor is called a sink.

A sequence $\left(e_{1}, e_{2}, \ldots, e_{k}\right)$ of edges of $G$ such that each edge has a common vertex with the previous edge, and the other vertex common to the next edge is called a chain of length $k$. Each equivalence class of the relation " $e_{i}$ is equal to $e_{j}$ or there exist a chain between $e_{i}$ and $e_{j}$ " is a connected component of the graph $G$.

A sequence $\left(e_{1}, e_{2}, \ldots, e_{k}\right)$ of arcs of $G$ such that for each $\operatorname{arc} e_{i}(1 \leq i<k)$ the end of $e_{i}$ is equal to the start of the arc $e_{i+1}$ is called a path of length $k$. Each equivalence class of the relation " $e_{i}$ is equal to $e_{j}$ or there exist a path between $e_{i}$ and $e_{j}$ " is a strongly connected component of the graph $G$.

A chain $\left(e_{1}, e_{2}, \ldots, e_{k}\right)$ of $G$ is called a cycle if the same edge does not occur more than once in the chain and if the 2 extremities of the chain coincide. A cycle $\left(e_{1}, e_{2}, \ldots, e_{k}\right)$ of $G$ is called a circuit if for each edge $e_{i}(1 \leq i<k)$, the end of $e_{i}$ is equal to the start of the edge $e_{i+1}$.

\footnotetext{
${ }^{9}$ See the diffn constraint that is defined in the list of global constraints.

${ }^{10}$ See the common constraint that is defined in the list of global constraints.
} 
The path, circuit and strongly connected component of a graph $G$ correspond to oriented concepts, while the edge, cycle and connected component are non-oriented concepts. However as it is reported in [BERGE 70 page 6] a nonoriented graph can be seen as an oriented graph where to each edge we associate the corresponding 2 arcs.

The rank function associated to the vertices $V(G)$ of a graph $G$ that does not contain any circuit is defined in the following way:

- the rank of the vertices that do not have any predecessor (i.e. the sources) is equal to 0 ,

- the rank $r$ of a vertex $v$ that is not a source is the length of longest path $\left(e_{1}, e_{2}, \ldots, e_{r}\right)$ such that the start of the $\operatorname{arc} e_{1}$ is a source and the end of $\operatorname{arc} e_{r}$ is the vertex $v$.

We list the different characteristics we consider for defining global constraints:

- NVERTEX:

- NARC:

- NARC_NO_LOOP:

- NSOURCE:

- NSINK:

- NCC:

- $\operatorname{NCC}(C O M P, v a l)$ :

- MIN_NCC:

- MAX_NCC:

- RANGE_NCC:

- NSCC:

- NSCC (COMP, val) :

- MIN_NSCC:

- MAX_NSCC:

- RANGE_NSCC:

- MAX_IN_DEGREE:

- NTREE:

- NTREe ( ATTR, COMP, val ):

- ORDER $(r, d, A T T R)$ :

- NSOURCE_EQ_NSINK:

- NSOURCE_GREATEREQ_NSINK: 1 if for each connected component the number of sources is greater or equal to

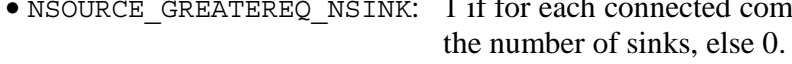

cardinality of the set $V(G)$.

cardinality of the set $E(G)$.

cardinality of the set $E(G)$ without considering the arcs that link the same vertices.

number of vertices that do not have any predecessor.

number of vertices that do not have any successor.

number of connected components of graph $G$.

number of connected components of graph $G$ such that the number of vertices $n v$ of a connected component verifies the comparison test $n v$ COMP val; val is a non-negative integer and COMP is one of the following test $=,>, \leq$, $<, \geq$.

number of vertices of the smallest connected component of graph $G$.

number of vertices of the largest connected component of graph $G$.

difference between the number of vertices of the largest connected component of graph $G$ and the number of vertices of the smallest connected component of graph $G$.

number of strongly connected components of graph $G$.

number of strongly connected components of graph $G$ such that the number of vertices $n v$ of a strongly connected component verifies the comparison test $n v$ COMP val; val is a non-negative integer and COMP is one of the following test $=,>, \leq,<, \geq$.

number of vertices of the smallest strongly connected component of graph $G$. number of vertices of the largest strongly connected component of graph $G$.

difference between the number of vertices of the largest strongly connected component of graph $G$ and the number of vertices of the smallest strongly connected component of graph $G$.

number of predecessors of the vertex of $G$ that has the maximum number of predecessors without counting an arc from a vertex to itself.

number of vertices of graph $G$ that do not belong to any circuit and for which at least one successor belongs to a circuit.

number of vertices of graph $G$ that do not belong to any circuit, and for which at least one successor belongs to a circuit, and for which value $v$ of attribute ATTR verifies the comparison test $v$ COMP val; COMP is one of the following test $=, \neq$.

$r$ and $d$ are integers and ATTR designates a specific attribute of the items associated to the vertices of graph $G$. Value of attribute ATTR associated to the vertices of rank $r$ (if several vertices have rank $r$ then we will get all corresponding values); $d$ if no such vertex exists.

1 if for each connected component the number of sources is equal to the number of sinks, else 0 . 
A last characteristic is computed on 2 graphs $G_{1}$ and $G_{2}$ that have the same set $V$ of vertices and the sets $E\left(G_{1}\right)$ and $E\left(G_{2}\right)$ of arcs. This characteristic corresponds to:

- DISTANCE:

cardinality of the set $\left(E\left(G_{1}\right)-E\left(G_{2}\right)\right) \cup\left(E\left(G_{2}\right)-E\left(G_{1}\right)\right)$. This corresponds to the number of arcs that belong to $E\left(G_{1}\right)$ but not to $E\left(G_{2}\right)$, plus the number of arcs that are in $E\left(G_{2}\right)$ but not in $E\left(G_{1}\right)$.

For directed hypergraphs, we will only consider the NARC characteristic.

\subsection{Description of simple global constraints}

A simple global constraint is defined by:

- A term $t\left(A_{1}, \ldots, A_{m}\right)$ where $t$ corresponds to the name of the constraint and $A_{1}, \ldots, A_{m}$ to its arguments, where each argument has a defined type, and where at least one of the arguments is a collection.

- A vertex generator, which takes an argument that is a collection and generates the vertices of the graph.

- An arc generator, which takes 2 arguments: the first one $n$ is the number of vertices of the arcs (usually $n=2$ ) and the second argument is one of the $n$ - ary constraints that were defined in section 2.2. .

- The graph properties that one wants to be verified on the final primal and dual graphs defined below are associated to an instantiated solution of the constraint.

We now introduce the notion of primal and dual graph according to a given instantiation of all variables that occur in the vertices of an initial graph that is associated to a global constraint. When these variables get instantiated then each $n$-ary constraint that is associated to an arc becomes either true or false. This creates a primal and a dual graph, which are respectively defined in the following way:

- The primal graph corresponds to the $n$-ary constraints that hold. A vertex of the initial graph belongs to the primal graph if it is involved in at least one $n$-ary constraint that holds. An arc belongs to the primal graph if the related constraint holds.

- The dual graph corresponds to the $n$-ary constraints that fail. A vertex of the initial graph belongs to the dual graph if this vertex is involved in at least one $n$-ary constraint that fails. An arc belongs to the dual graph if the related constraint fails.

Since some constraint request it, we introduce a second type of dual graph that is defined in the following way:

- As in the previous case, the dual graph of type 2 corresponds to the $n$-ary constraints that fail. A vertex of the initial graph belongs to the dual graph of type 2 if that vertex is only involved in $n$-ary constraints that fail. An arc belongs to the dual graph of type 2 if its extremities are vertices of the dual graph of type 2 and if the constraint related to the arc fails.

We use the following notations in order to ask for a graph propertie on the primal or on the dual graph:

- A graph propertie $\mathrm{P}$ on the primal graph will be denoted P(PRIMAL) or simply $\mathrm{P}$,

- A graph propertie $\mathrm{P}$ on the dual graph of type 1 will be denoted P(DUAL1) or simply P(DUAL),

- A graph propertie $\mathrm{P}$ on the dual graph of type 2 will be denoted P(DUAL2).

Note the important difference between this way of defining a constraint and the classical approach, where one specifies a network of constraints [DECHTER \& PEARL 87], [DECHTER, MEIRI \& PEARL 91] which all have to hold. Let us now give a small example of how one can define the alldifferent $\left(\left[X_{1}, \ldots, X_{n}\right]\right)$ constraint that holds if all variables $X_{1}, \ldots, X_{n}$ are pairwise different:

- The vertices of the graph correspond to the variables $X_{1}, \ldots, X_{n}$,

- The arity of the constraint that is associated to each arc is 2 ,

- The constraint that is associated to each arc is the equality constraint,

- The arcs of the initial directed graph are construct with the CLIQUE arcs generator: each vertex is linked to each other vertex,

- The graph properties that one wants to be verified on the final primal graph that is associated to an instantiated solution corresponds to the fact that we want to have exactly $n$ strongly connected components.

The next figure shows the initial graph, the primal and dual graphs of first type that are associated to the instantiation $X_{1}=5, X_{2}=5, X_{3}=3$ and $X_{4}=8$. For the initial graph we indicate in each vertex the indices of the corresponding variable, while we give the value of the variables in the case of the primal and dual graphs. Since the primal graph has only 3 strongly connected components, the constraint alldifferent $([5,5,3,8])$ is not satisfied. 


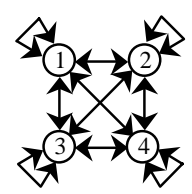

Initial graph

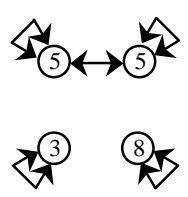

Primal graph

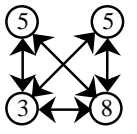

Dual graph

Figure 2. The different graphs associated to alldifferent $\left(\left[X_{1}=5, X_{2}=5, X_{3}=3, X_{4}=8\right]\right)$.

\subsection{Description of dynamic global constraints}

The purpose of a dynamic global constraint is to enforce a condition on different subsets of variables, which are not known in advance. These constraints occur very often in practice and are hard to express since one cannot use a classical constraint for which it is required to provide all variables. One good example of dynamic global constraint is the cumulative ${ }^{11}$ constraint where one wants to impose the sum of some variables to be less or equal than a given limit. In this case, each set of variables is defined by the height of the different tasks that overlap a given instant $i$. Since the origins of the tasks are not initially fixed, we don't know in advance which task will overlap a given instant and so, we cannot state any sum constraint initially. A dynamic global constraint is defined in exactly the same way that a simple global constraint, except that we can eventually omit the graph properties, and that we have to provide the 2 following additional informations:

- A generator of sets of vertices, which takes as argument the final primal graph and produces different sets of vertices.

- An elementary or a simple global constraint that one has to apply on each set created by the previous generator.

We now describe the different generators of sets of vertices that we provide:

- The PATH $(L)$ generates all paths of $L$ distinct vertices of the primal graph,

- The PATH (MAX) generates all maximum paths of distinct vertices of the primal graph,

- The CC (MIN) generates all connected components of no less than MIN vertices of the primal graph,

- The ALL_CC (MIN) generates one set that corresponds to all connected components of no less than MIN vertices of the primal graph,

- The SCC (MIN) generates all strongly connected components of no less than MIN vertices of the primal graph,

- The ALL_SCC (MIN) generates one set that corresponds to all strongly connected components of no less than MIN vertices of the primal graph,

- The CLIQUE (MAX) generates all maximum cliques of the primal graph.

The PATH generator can only be used when the definition of the global constraint implies one of the following conditions:

- Discarding the loops, all the vertices of the primal graph have exactly one predecessor.

- Discarding the loops, all the vertices of the primal graph have exactly one successor.

The CLIQUE generator can only be applied when the primal graph is a subclass of intersection graphs [McKEE \& McMORRIS 99] for which one can compute the maximum cliques in a polynomial time according to the number of vertices.

Let us now give a small example of how one can define the cumulative(Tasks, Limit) constraint, where Tasks is a collection of the form collection(Index-int, Origin-dvar,Duration-dvar,Height-dvar) and where Limit is a non-negative integer. The constraint holds if the following condition is true:

$$
\forall i \in \mathbb{I N} \sum_{j \mid \text { Origin }_{j} \leq i<\text { Origin }_{j}+\text { Duration }_{j}} \text { Height }_{j} \leq \text { Limit } .
$$

The cumulative dynamic global constraint is defined by:

- The vertices of the graph correspond to the different tasks of the collection Tasks,

- The arity of the constraint that is associated to each arc is 2 ,

- The constraint that is associated to each arc is the overlapping constraint that holds if the corresponding 2 tasks intersect in time,

- The arcs of the initial directed graph are constructed with the CLIQUE $(\neq)$ arcs generator: each vertex is linked to each other vertex except itself,

- The sets generator is CLIQUE (MAX). Since the arc constraint is the overlapping constraint, the primal graph will be an interval graph on which one can compute the different maximum cliques in a polynomial time according to the number of tasks.

\footnotetext{
${ }^{11}$ The cumulative constraint is defined in appendix 1.
} 
- The dynamic constraint is that the sum of the heights of the tasks that are in a maximum clique should not exceed Limit .

The next figure shows the initial graph (A), the primal and the maximum cliques (B) that are associated to the following instance:

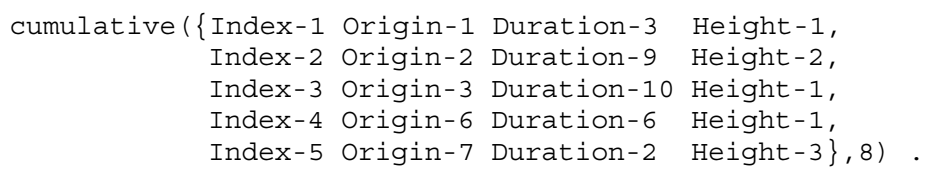

We indicate in each vertex the index of the corresponding task. On the primal graph an ellipse encloses each maximum clique. The constraint is satisfied, since for the 2 maximum cliques, the sum of the heights of the involved tasks does not exceed the maximum limit 8 .

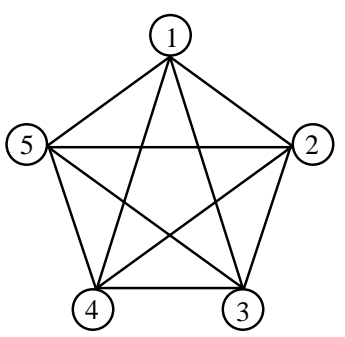

(A)

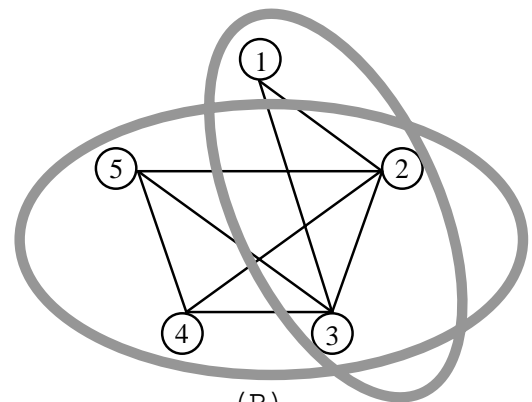

(B)

Figure 3. Initial and primal graphs associated to the cumulative instance

In the next paragraph, we show how one can redefine nearly all the currently existing global constraints that are predefined in current constraint systems CHIP [DINCBAS, VAN HENTENRYCK, SIMONIS, AGGOUN, GRAF \& BERTHIER 88], CLAIRE [CASEAU \& LABURTHE 96a], Ilog Solver [ILOG 99], Oz [SMOLKA 96] and SICStus implementation of clp(FD) [CARLSSON, OTTOSSON \& CARLSON 97]. Moreover we come out with a lot of new powerful global constraints that can be naturally defined within this new framework.

\section{A catalogue of global constraints}

The purpose of this section is to show how one can express with the previous description language most of the existing global constraints and how one can come up with a variety of new interesting global constraints. This corresponds in fact to explore the global constraint space that is depicted by the next figure.

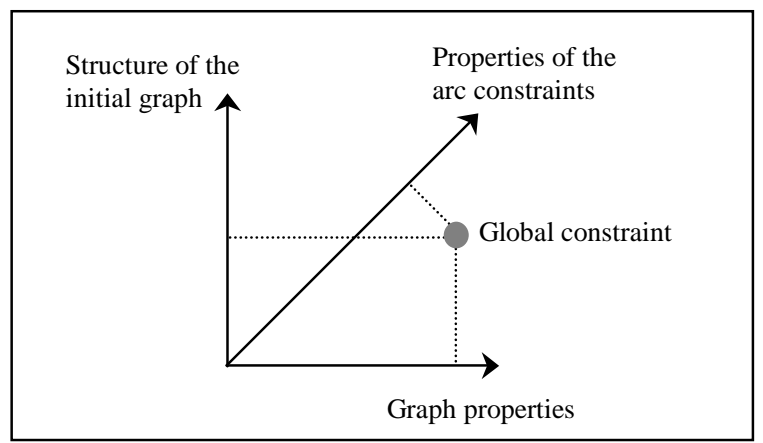

Figure 4. The different dimensions of the global constraint "space".

A simple global constraint can be seen as an interaction between the structure of the initial primal graph, the properties of the edge constraint and the graph properties that have to hold on the final graph. For the dynamic global constraints we have 2 additional dimensions, which respectively are the kind of set generators and the property of the constraint that has to hold on each set. The following slots describe each simple global constraint:

- Origin:

describes the origin of the constraint that corresponds to the (to our knowledge) first known constraint system or paper that introduces it. When this constraint does not already exist we mention it with the keyword NEW, even when a similar problem or model was presented using something else outside the constraint community.

- Constraint: gives a typical name and the different arguments of the constraint.

- Arguments: describes for each argument its type.

- Restriction(s): $\quad$ provides necessary conditions on the arguments of the constraint. 
- Vertex input:

indicates which of the arguments of the constraint are used for the vertex generator. These arguments are collections that are passed to the vertex generator in order to generate the vertices of the graph that is associated to the global constraint.

- Vertex generator: gives the vertex generator that is used in order to generate the vertices of the graph.

- Arc generator: describes the arc generator that is used in order to generate the arcs of the graph.

- Arc arity: gives the number of vertices of the arcs of the graph; this corresponds to the arity of the elementary constraints that are associated to each arc.

- Arc constraint: gives the elementary constraint that is associated to each arc of the graph.

- Graph property(ies): provides all graph properties that one want to have on the primal and/or dual graphs that is associated to an instantiated solution.

- Purpose: indicates what is the purpose of the constraint.

- Example(s): $\quad$ provides one or several examples where we give a ground solution for which the constraint holds.

- Comment: $\quad$ explains the description (i.e. vertex generator, arc generator, graph constraint) of the global constraint.

- Usage: $\quad$ gives possible potential utilisation of the constraint.

- Remark: $\quad$ gives a specific remark on the constraint.

For each dynamic global constraint we provide the additional 2 slots:

- Sets: describe the way sets of vertices are generated.

- Dynamic constraint: give the elementary constraint or the simple global constraint that has to hold for every set of vertices generated by the previous slot.

We have regrouped the different global constraints under the following 11 categories:

- Order constraints:

- Value constraints:

- Bipartite constraints:

- Value partitioning constraints:

- Sliding sequence constraints:

- Timetabling constraints:

- Neighbourhood constraints:

- Graph constraints:

- Scheduling constraints:

- Geometrical constraints:

- Miscellaneous constraints: minimum or maximum value according to some defined ordering relation, how variables can take values, constraint involving a bipartite graph,

partition values according to an equivalence relation, elementary constraint on sliding sequences of variables that partially overlap, constraints expressing rules how people can work, proximity between 2 series of variables according to a given elementary constraint, graph-partitioning constraints, resource-scheduling constraints, placement, alignment and crossing constraints, constraints that do not fit in one of the previous categories. 


\subsection{Order constraints}

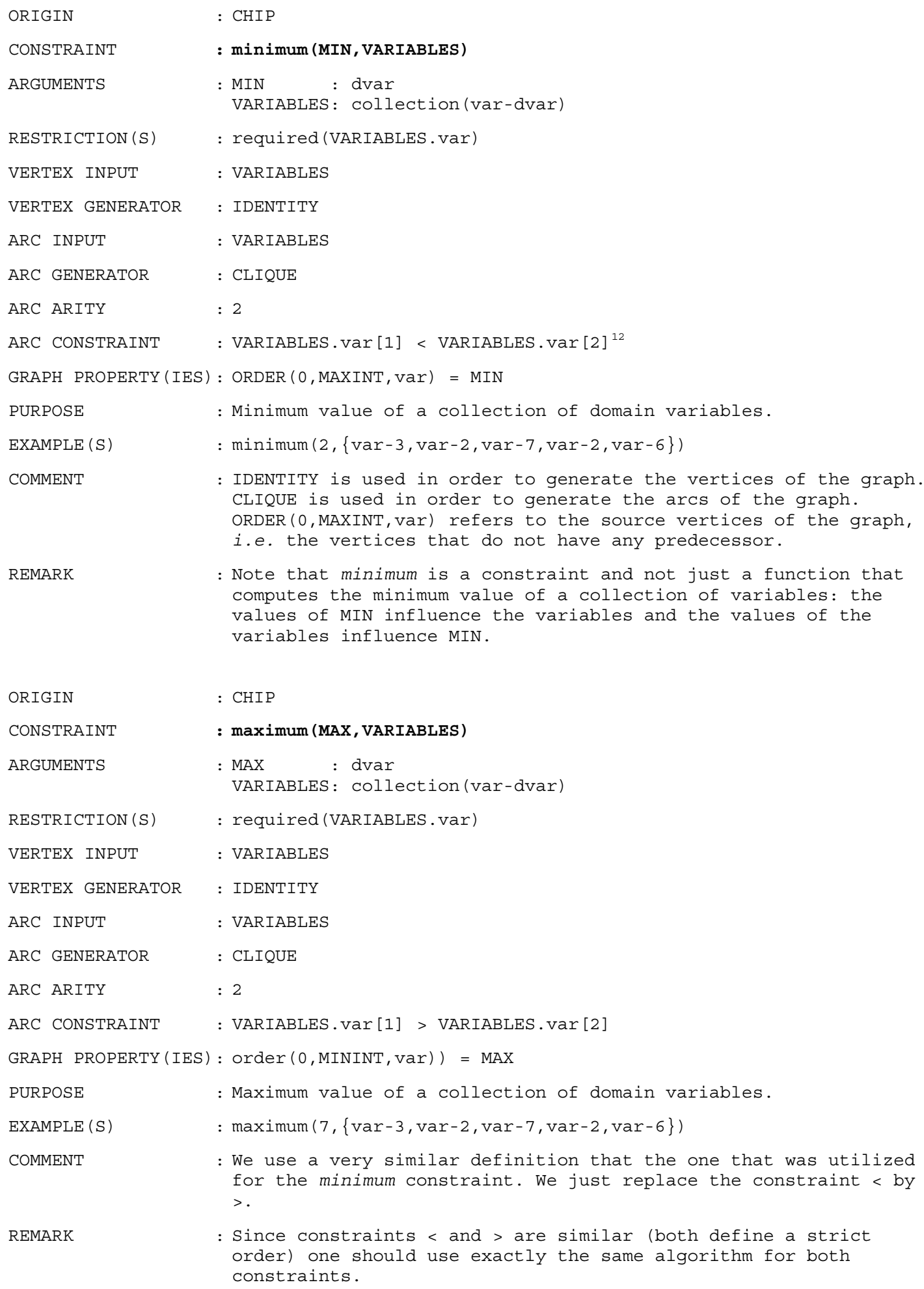

REMARK

: Note that minimum is a constraint and not just a function that computes the minimum value of a collection of variables: the values of MIN influence the variables and the values of the variables influence MIN. ${ }^{12}[1]$ refers to the initial node of an arc constraint and [2] to the destination node. This notation will be used for all
the arc constraints. 


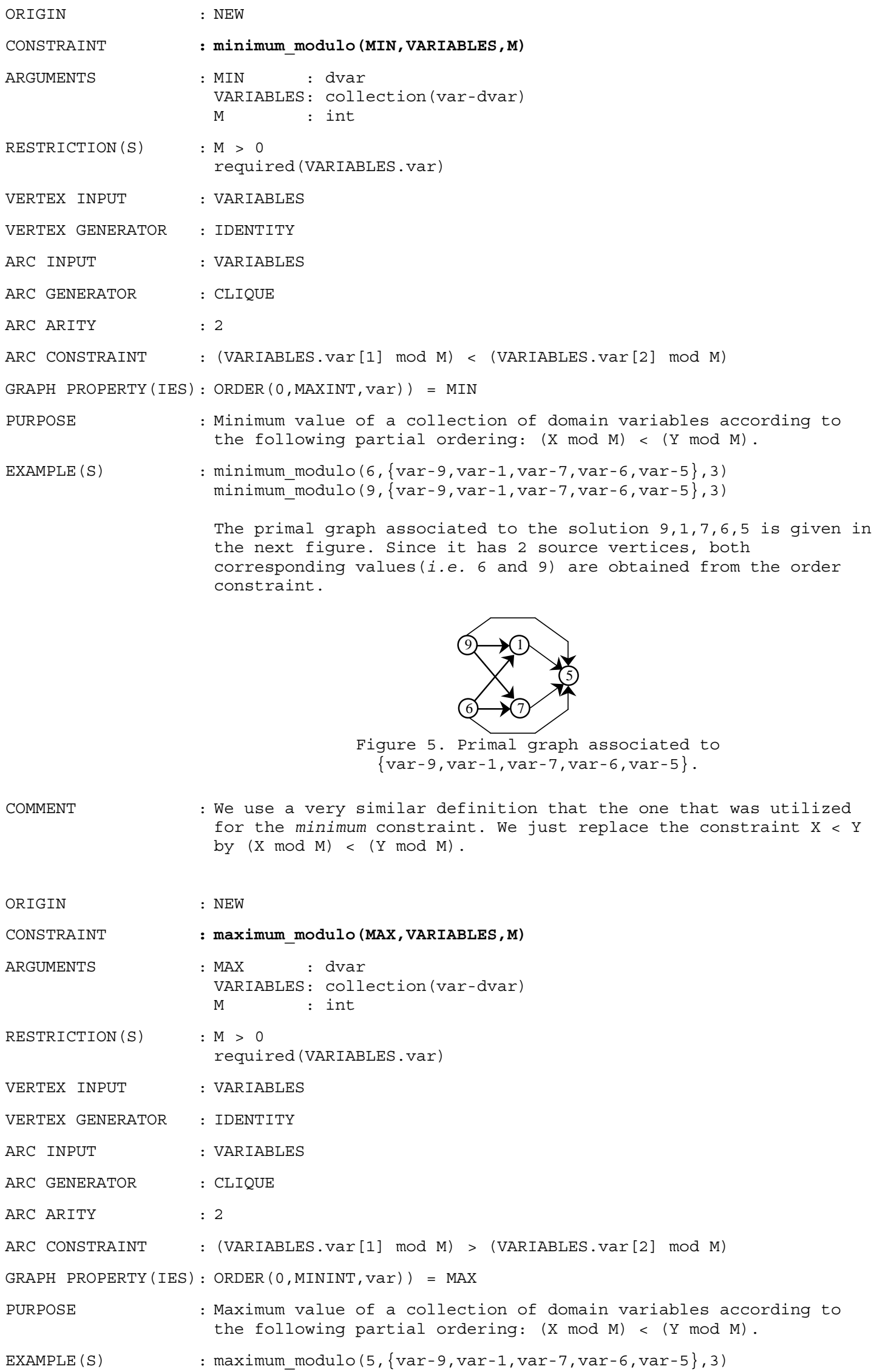

Figure 5. Primal graph associated to $\{\operatorname{var}-9, \operatorname{var}-1, \operatorname{var}-7, \operatorname{var}-6, \operatorname{var}-5\}$.

COMMENT

: We use a very similar definition that the one that was utilized for the minimum constraint. We just replace the constraint $X<Y$ by $(\mathrm{X} \bmod \mathrm{M})<(\mathrm{Y} \bmod \mathrm{M})$. 

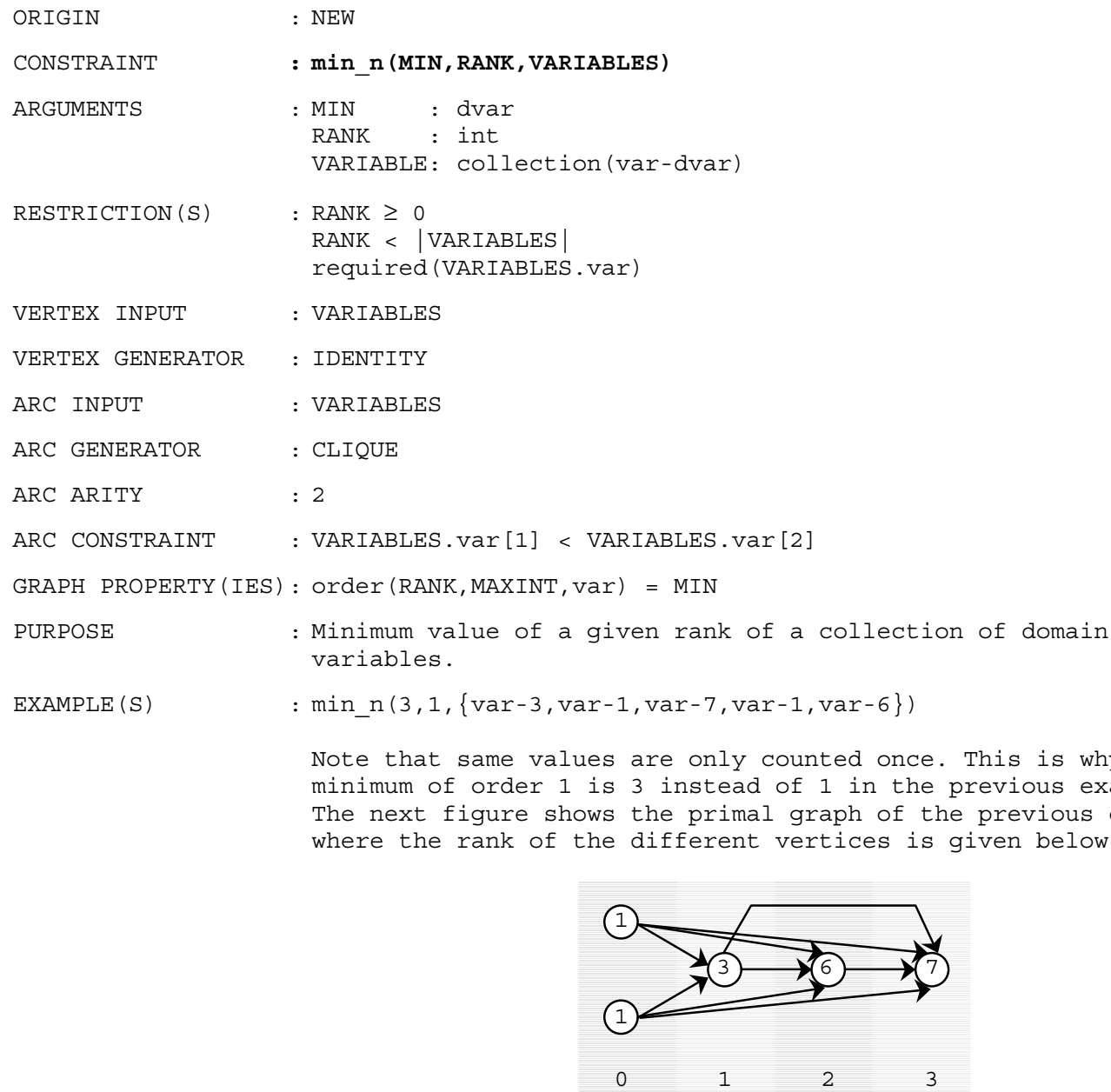

Figure 6. Primal graph associated to the min $n$ constraint and corresponding ranks. 


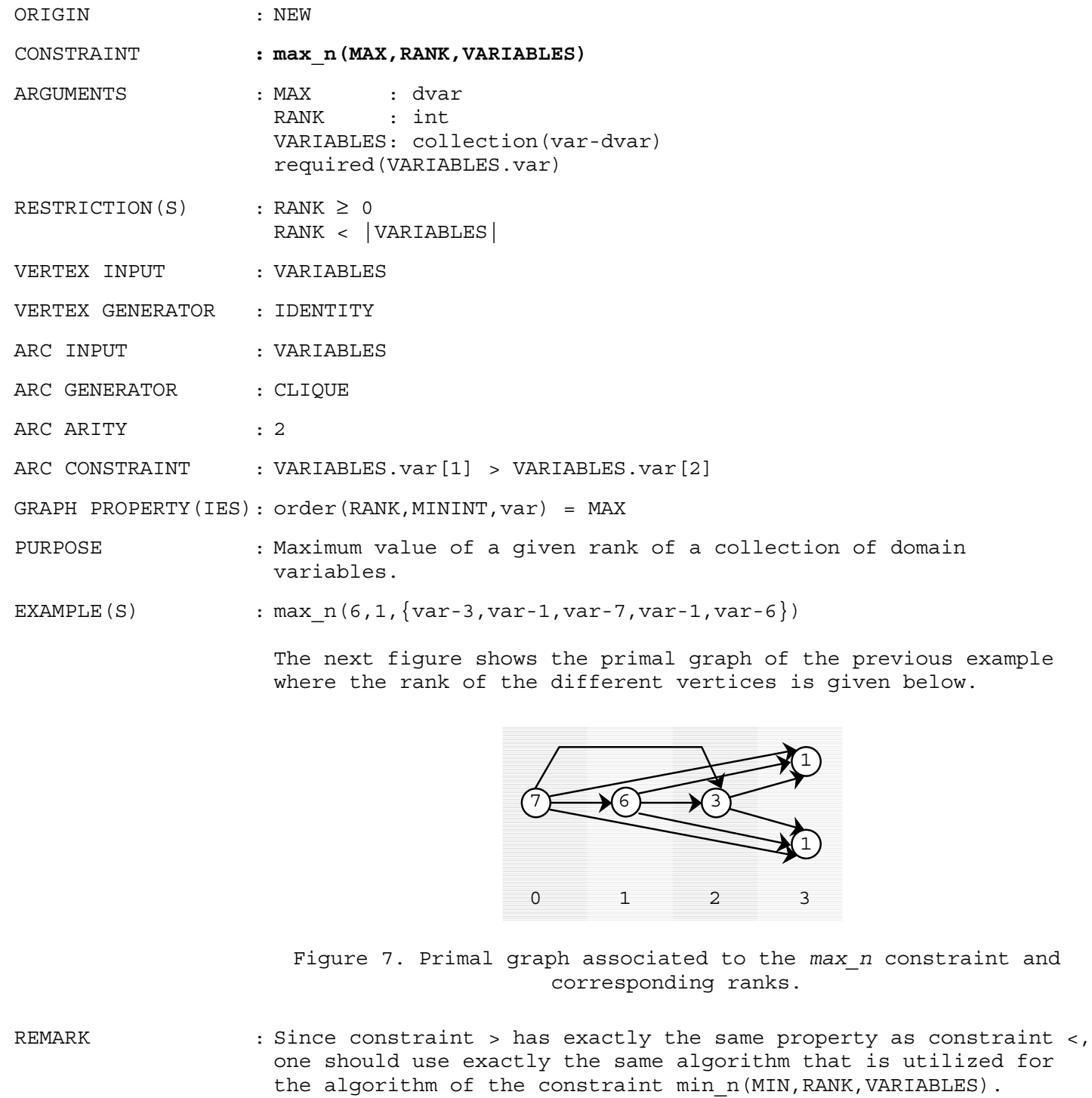

$\begin{array}{llll}0 & 1 & 2 & 3\end{array}$

Figure 7. Primal graph associated to the max_n constraint and corresponding ranks.

REMARK

: Since constraint > has exactly the same property as constraint <, one should use exactly the same algorithm that is utilized for the algorithm of the constraint min $n$ (MIN, RANK, VARIABLES). 


\subsection{Value constraints}

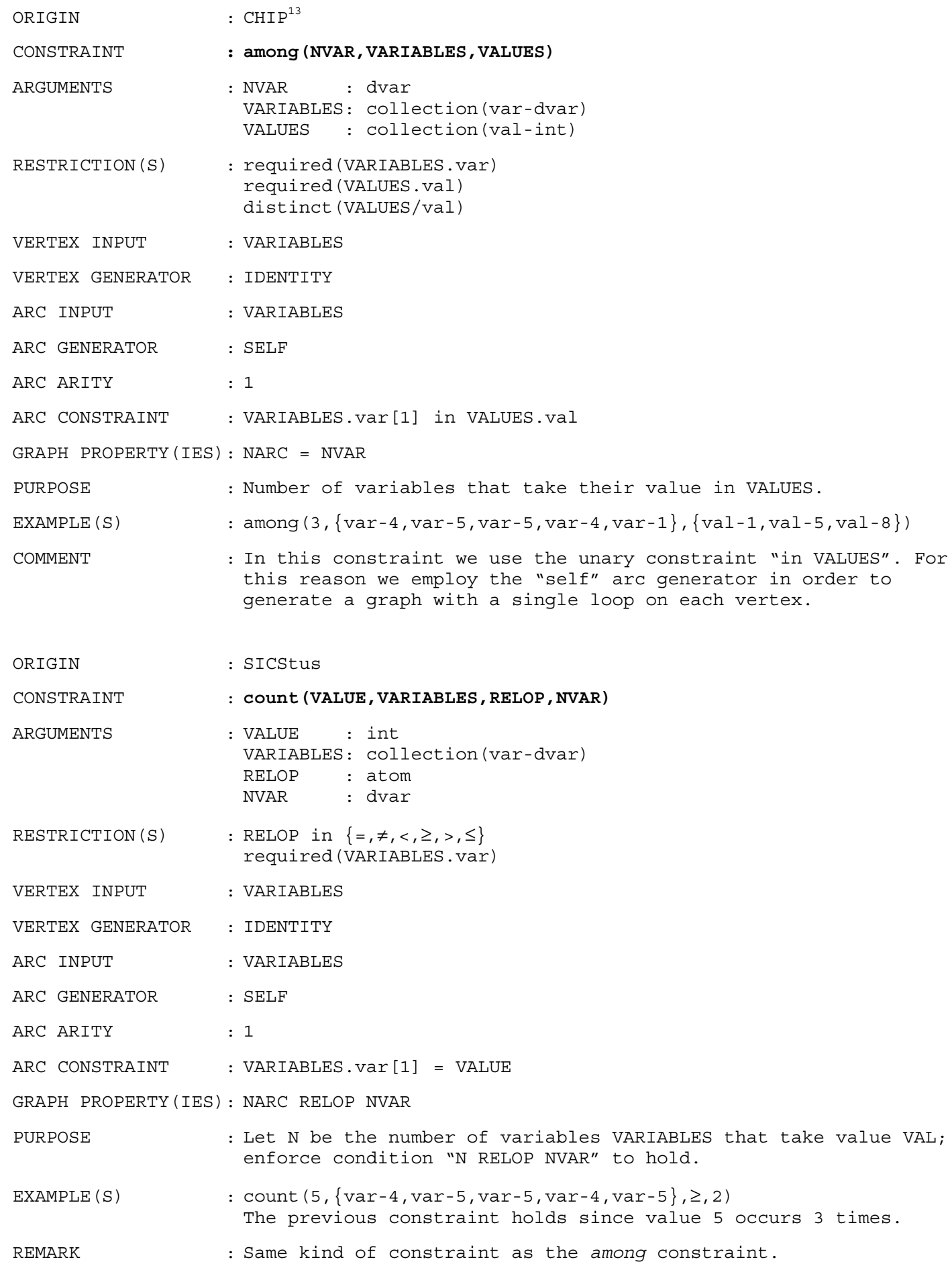

${ }^{13}$ A similar constraint called "between" was introduced in 1990 in CHIP. 


\begin{tabular}{|c|c|}
\hline ORIGIN & : NEW \\
\hline CONSTRAINT & : balance (BALANCE, VARIABLES) \\
\hline ARGUMENTS & $\begin{array}{l}\text { BALANCE : dvar } \\
\text { VARIABLES: collection(var-dvar) }\end{array}$ \\
\hline RESTRICTION (S) & $\begin{array}{l}\text { BALANCE } \geq 0 \\
\text { BALANCE } \leq \mid \text { VARIABLES } \\
\text { required (VARIABLES.var) }\end{array}$ \\
\hline VERTEX INPUT & : VARIABLES \\
\hline VERTEX GENERATOR & : IDENTITY \\
\hline ARC INPUT & : VARIABLES \\
\hline ARC GENERATOR & : CLIQUE \\
\hline ARC ARITY & $: 2$ \\
\hline ARC CONSTRAINT & $:$ VARIABLES.var[1] = VARIABLES.var [2] \\
\hline GRAPH PROPERTY (IES) & $:$ RANGE_NSCC $=$ BALANCE \\
\hline PURPOSE & $\begin{array}{l}\text { Difference between the number of occurrence of the value that } \\
\text { occurs the most and the value that occurs the least. }\end{array}$ \\
\hline EXAMPLE ( $\mathrm{S}$ ) & : balance $(2,\{\operatorname{var}-3, \operatorname{var}-1, \operatorname{var}-7, \operatorname{var}-1, \operatorname{var}-1\})$ \\
\hline & $\begin{array}{l}\text { In this example, values } 1,3 \text { and } 7 \text { are respectively used } 3,1 \text { and } \\
1 \text { times. BALANCE is assigned to the difference between the } \\
\text { maximum and minimum number of the previous occurrences (i.e. } 3 \text { - } \\
\text { 1). }\end{array}$ \\
\hline USAGE & $\begin{array}{l}\text { One application of this constraint is to enforce a balanced } \\
\text { assignment of values, no matter how many distinct values will be } \\
\text { used. In this case one will "push" on the maximum value of the } \\
\text { first argument of the balance constraint. }\end{array}$ \\
\hline
\end{tabular}




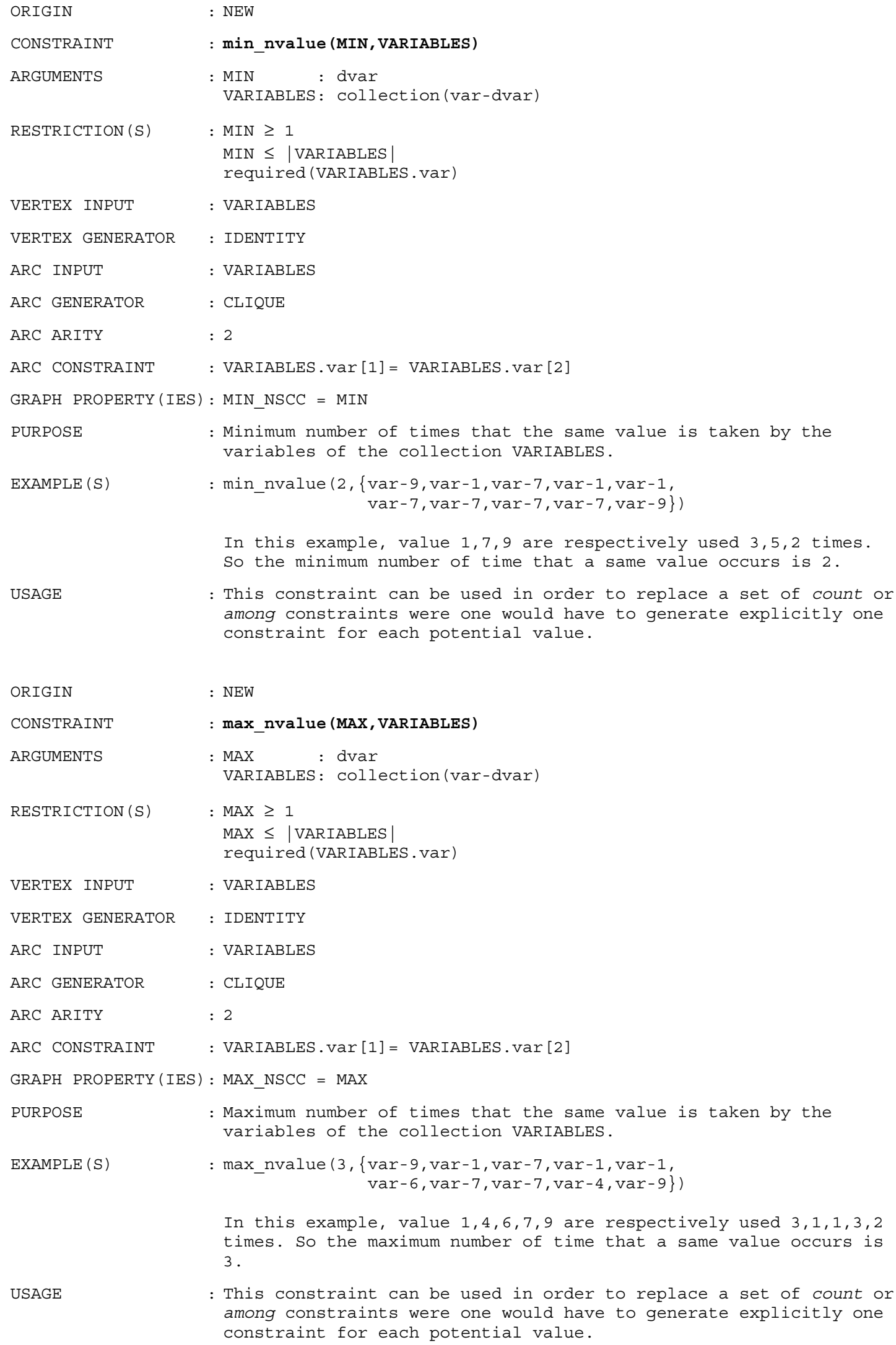

USAGE

: This constraint can be used in order to replace a set of count or among constraints were one would have to generate explicitly one constraint for each potential value.

USAGE

: This constraint can be used in order to replace a set of count or among constraints were one would have to generate explicitly one constraint for each potential value. 


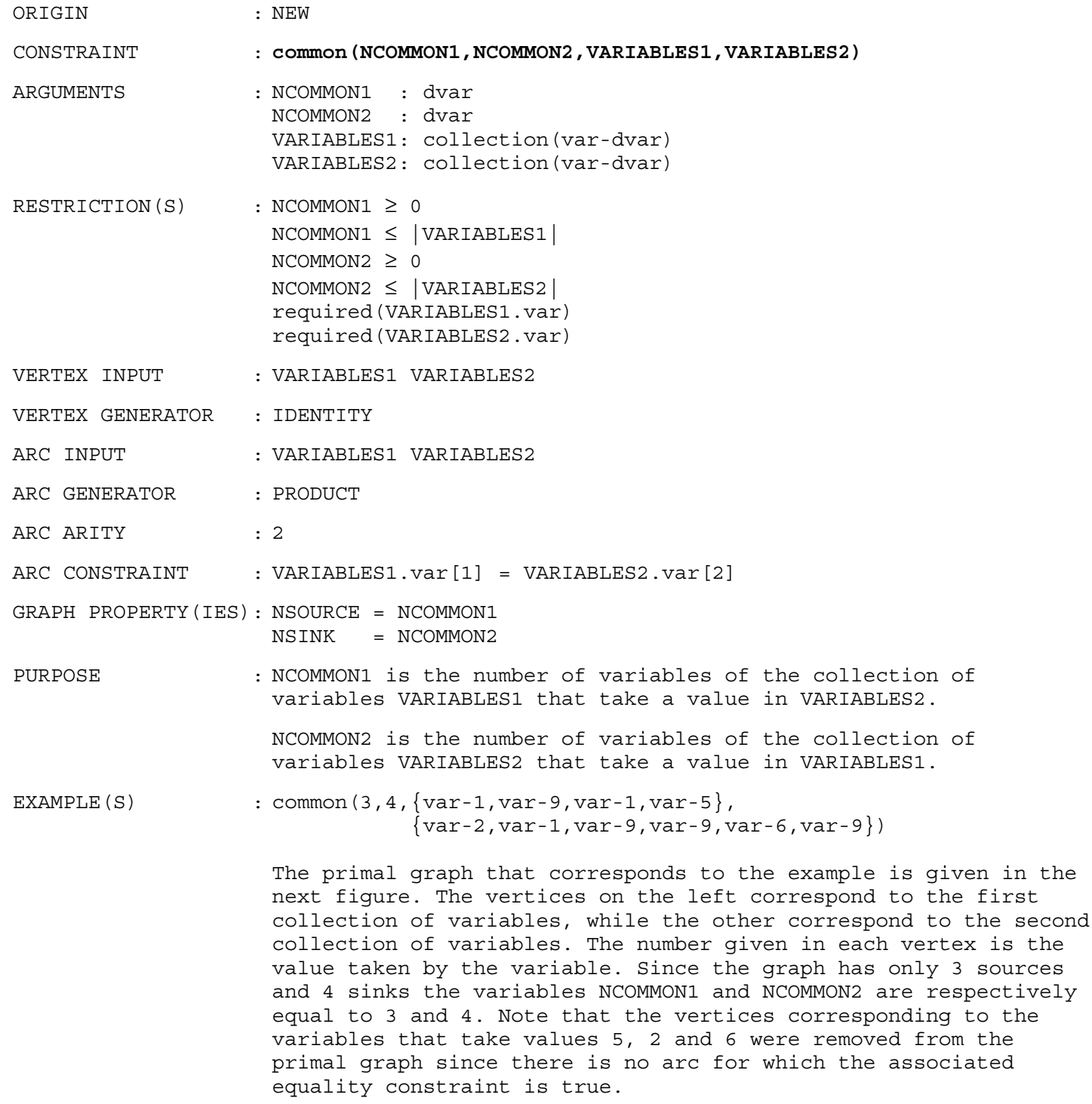

EXAMPLE (S)

: $\operatorname{common}(3,4,\{\operatorname{var}-1, \operatorname{var}-9, \operatorname{var}-1, \operatorname{var}-5\}$,

$\{\operatorname{var}-2, \operatorname{var}-1, \operatorname{var}-9, \operatorname{var}-9, \operatorname{var}-6, \operatorname{var}-9\})$

The primal graph that corresponds to the example is given in the next figure. The vertices on the left correspond to the first collection of variables, while the other correspond to the second collection of variables. The number given in each vertex is the value taken by the variable. Since the graph has only 3 sources and 4 sinks the variables NCOMMON1 and NCOMMON2 are respectively equal to 3 and 4 . Note that the vertices corresponding to the variables that take values 5,2 and 6 were removed from the primal graph since there is no arc for which the associated equality constraint is true.

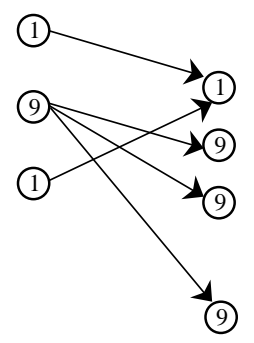

Figure 8. Bipartite primal graph. 


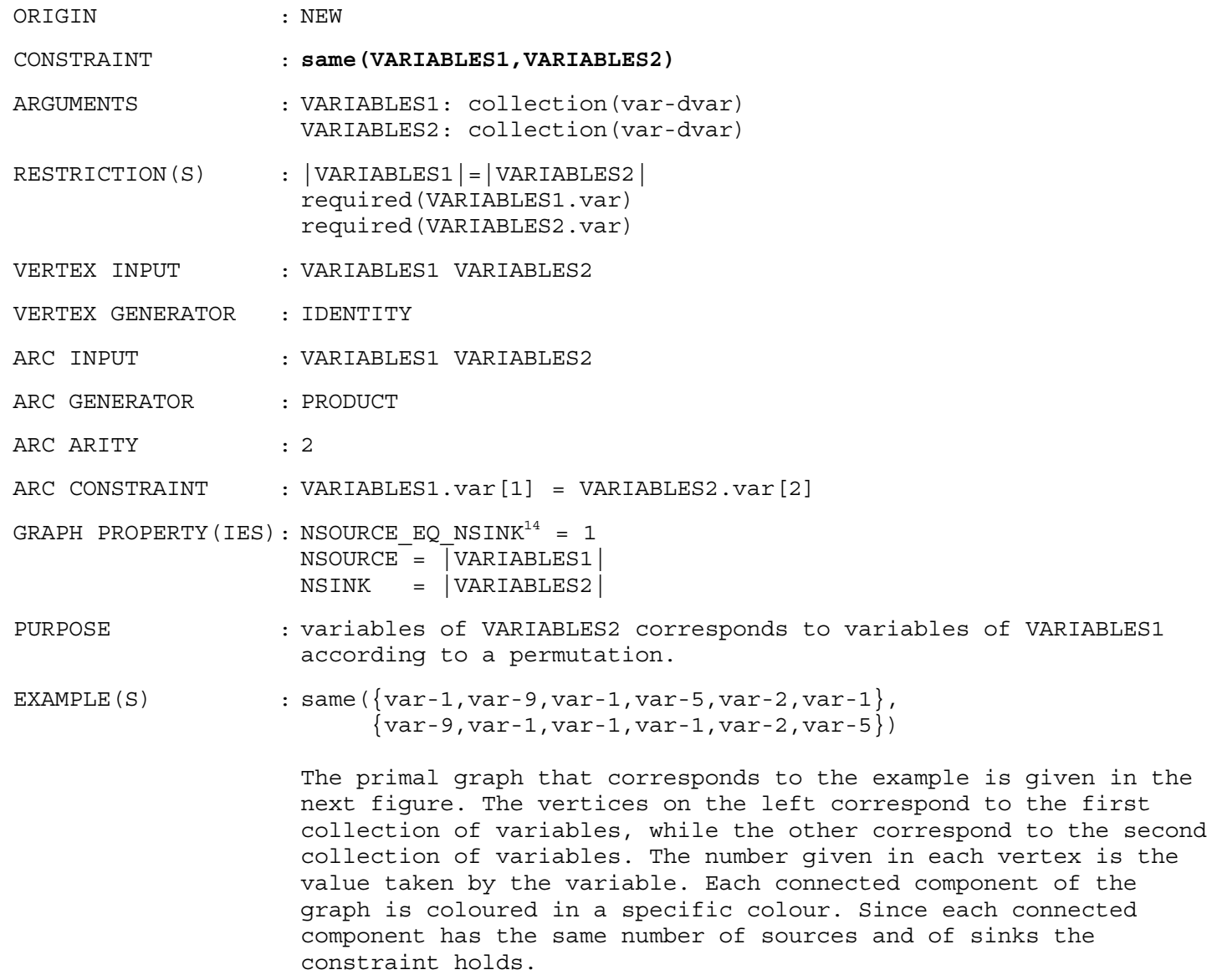

PURPOSE

$\operatorname{EXAMPLE}(\mathrm{S})$

: variables of VARIABLES2 corresponds to variables of VARIABLES1 according to a permutation.

: same ( $\{\operatorname{var}-1, \operatorname{var}-9, \operatorname{var}-1, \operatorname{var}-5, \operatorname{var}-2, \operatorname{var}-1\}$,

$$
\{\operatorname{var}-9, \operatorname{var}-1, \operatorname{var}-1, \operatorname{var}-1, \operatorname{var}-2, \operatorname{var}-5\})
$$

The primal graph that corresponds to the example is given in the next figure. The vertices on the left correspond to the first collection of variables, while the other correspond to the second collection of variables. The number given in each vertex is the value taken by the variable. Each connected component of the graph is coloured in a specific colour. Since each connected component has the same number of sources and of sinks the constraint holds.

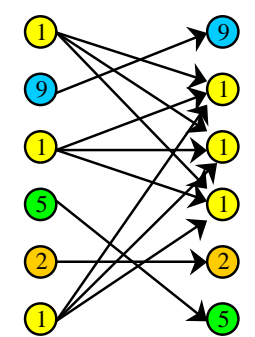

Figure 9. The 4 connected components of the bipartite primal graph.

REMARK

\begin{abstract}
: The same constraint is a relaxed version of the sort constraint introduced in [ZHOU 97] and after in [GUERNALEC \& COLMERAUER 97]. We don't give the permutation that relate the 2 collections of variables and don't enforce the second collection of variables to be sorted in increasing order.
\end{abstract}

\footnotetext{
${ }^{14} 1$ if for each connected component the number of sources is equal to the number of sinks, else 0 .
} 


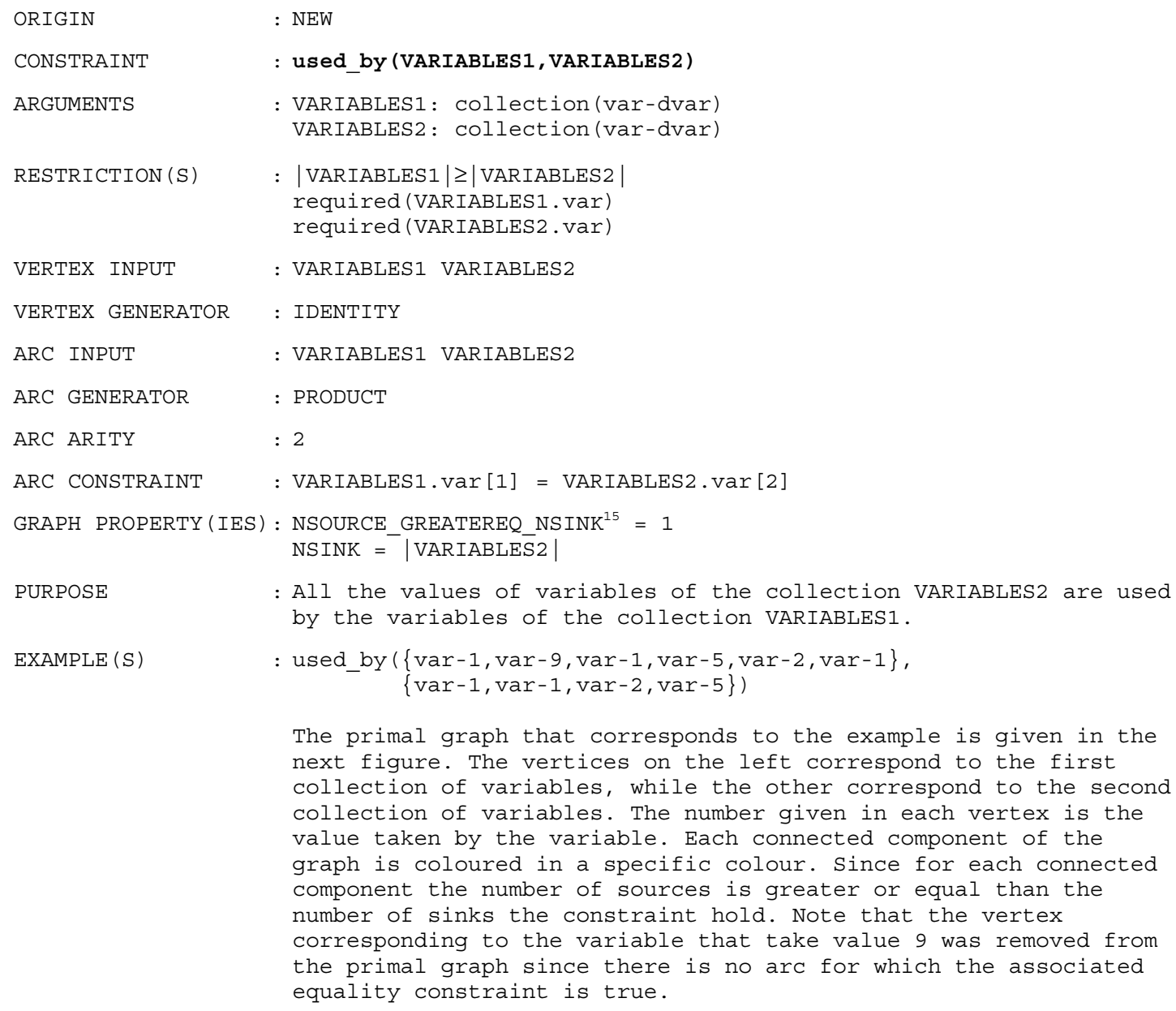

$\operatorname{EXAMPLE}(\mathrm{S})$

: used_by ( $\{\operatorname{var}-1, \operatorname{var}-9, \operatorname{var}-1, \operatorname{var}-5, \operatorname{var}-2, \operatorname{var}-1\}$,

$\{\operatorname{var}-1, \operatorname{var}-1, \operatorname{var}-2, \operatorname{var}-5\})$

The primal graph that corresponds to the example is given in the next figure. The vertices on the left correspond to the first collection of variables, while the other correspond to the second collection of variables. The number given in each vertex is the value taken by the variable. Each connected component of the graph is coloured in a specific colour. Since for each connected component the number of sources is greater or equal than the number of sinks the constraint hold. Note that the vertex corresponding to the variable that take value 9 was removed from the primal graph since there is no arc for which the associated equality constraint is true.

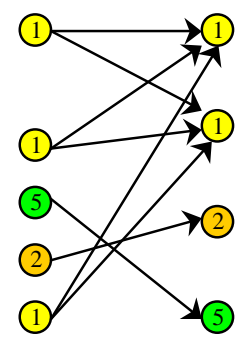

Figure 10. The 3 connected components of the bipartite primal graph.

\footnotetext{
151 if for each connected component the number of sources is greater or equal to the number of sinks, else 0 .
} 


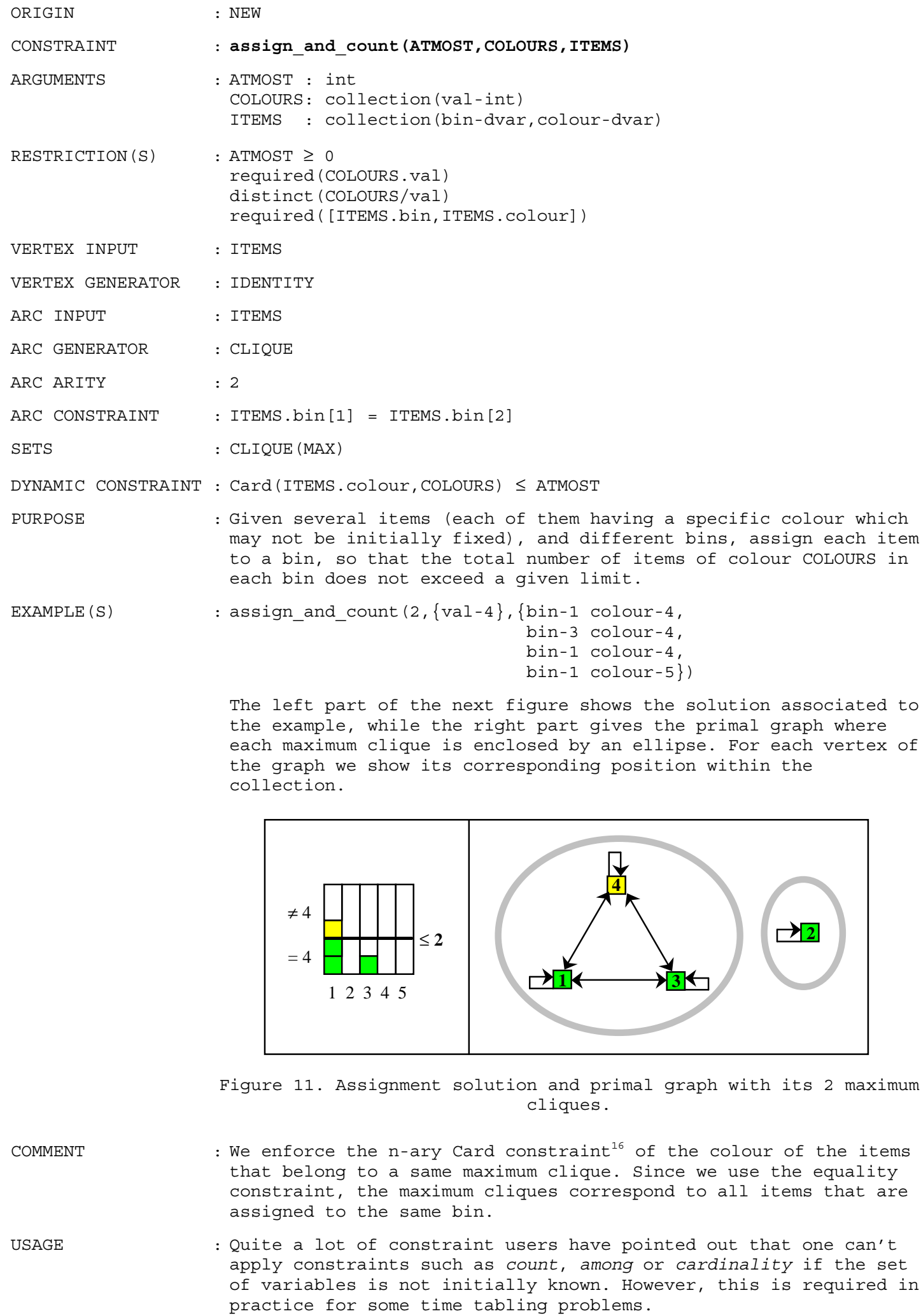

EXAMPLE (S)

: assign_and_count $(2,\{$ val-4 $\},\{$ bin-1 colour-4, bin-3 colour-4, bin-1 colour-4,

The left part of the next figure shows the solution associated to the example, while the right part gives the primal graph where each maximum clique is enclosed by an ellipse. For each vertex of the graph we show its corresponding position within the collection.

Figure 11. Assignment solution and primal graph with its 2 maximum cliques.

COMMENT

: We enforce the n-ary Card constraint ${ }^{16}$ of the colour of the items that belong to a same maximum clique. Since we use the equality constraint, the maximum cliques correspond to all items that are assigned to the same bin.

USAGE

: Quite a lot of constraint users have pointed out that one can't apply constraints such as count, among or cardinality if the set of variables is not initially known. However, this is required in practice for some time tabling problems.

\footnotetext{
${ }^{16}$ Refers to the Card constraint that was introduced in the paragraph 2.2.1. . It enforces the number of variables that take some specific values to be between 2 given limits. In our example we use only the upper limit.
} 


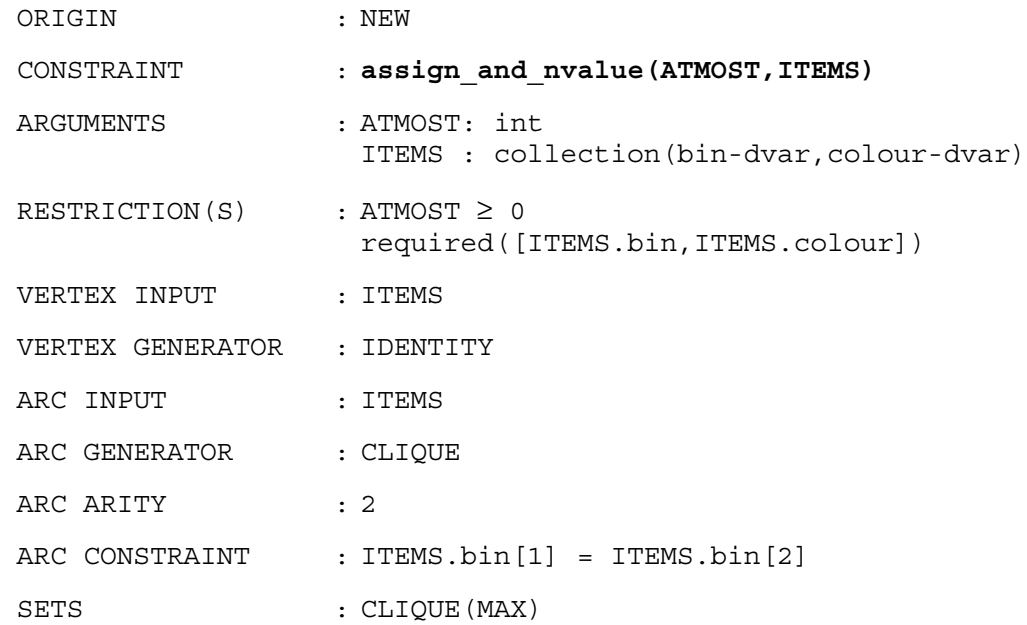

The left part of the next figure shows the solution associated to the example, while the right part gives the primal graph where the 2 maximum cliques are indicated by an ellipse. For each vertex of the graph we show its corresponding position within the collection.

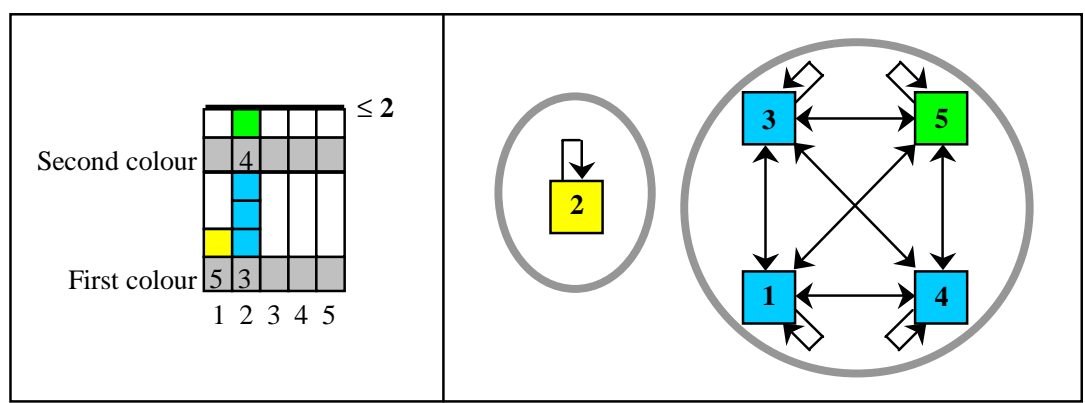

Figure 12. An assignment with at most 2 distinct colors in parallel and the related primal graph with its maximum cliques.

COMMENT

: We enforce the non-primitive constraint nvalue ${ }^{17}$ on the items that belong to a same maximum clique. Since we use the equality constraint, the maximum cliques correspond to all items that are assigned to the same bin.

USAGE

: Let us give 2 examples where this constraint is useful:

- Quite often, in bin-packing problems, each item has a specific type, and one wants to assign items of similar type to each bin.

- In a vehicle routing problem, one wants to restrict the number of towns visited by each vehicle. Note that several customers may be located at the same town. In this example, each bin would correspond to a vehicle, each item would correspond to a visit to a customer, and the colour of an item would be the location of the corresponding customer.

\footnotetext{
${ }^{17}$ Refers to the nvalue constraint that was defined as a non-primitive constraint. It constrains the number of distinct values to be in a specified range.
} 


\subsection{Bipartite constraints}

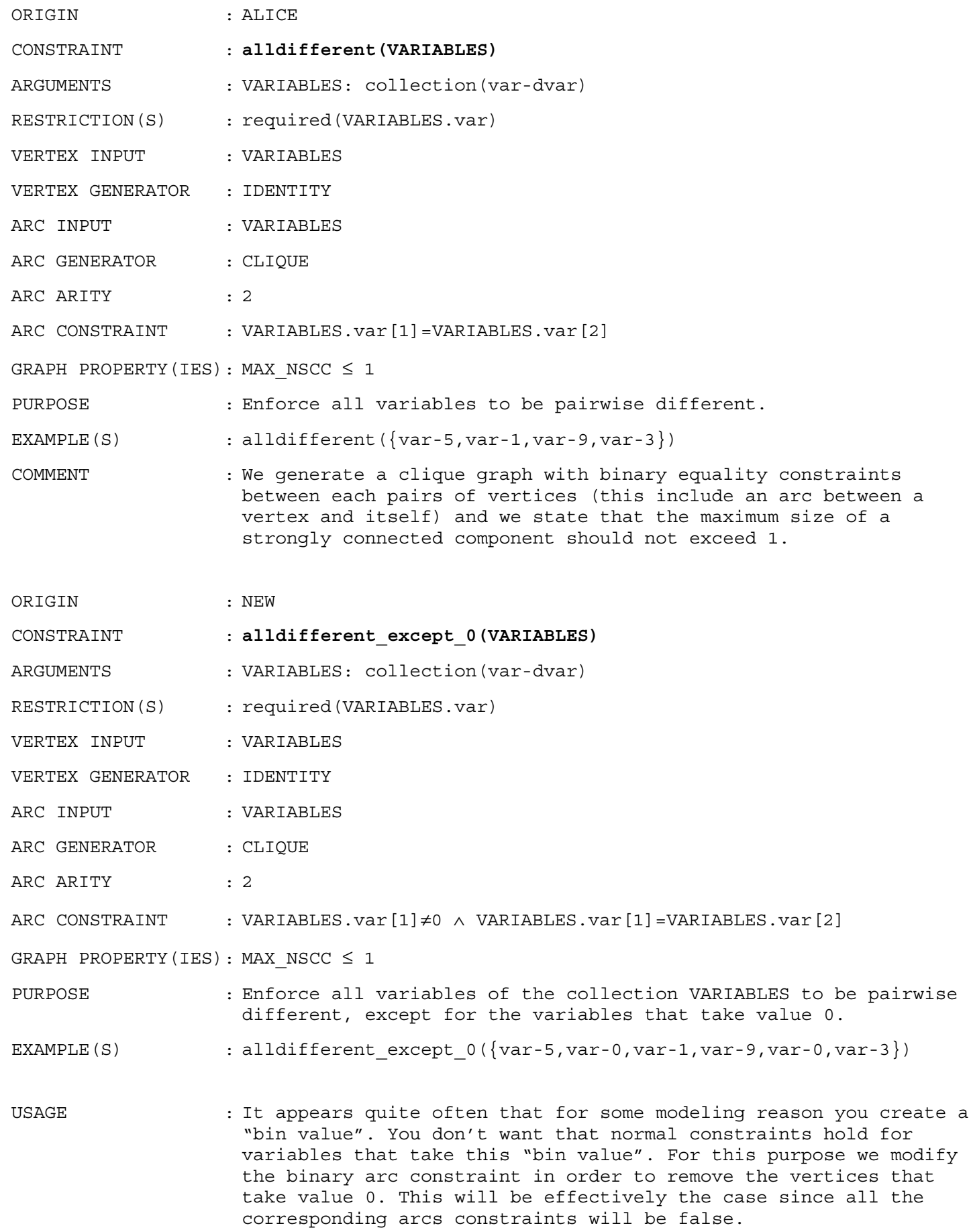




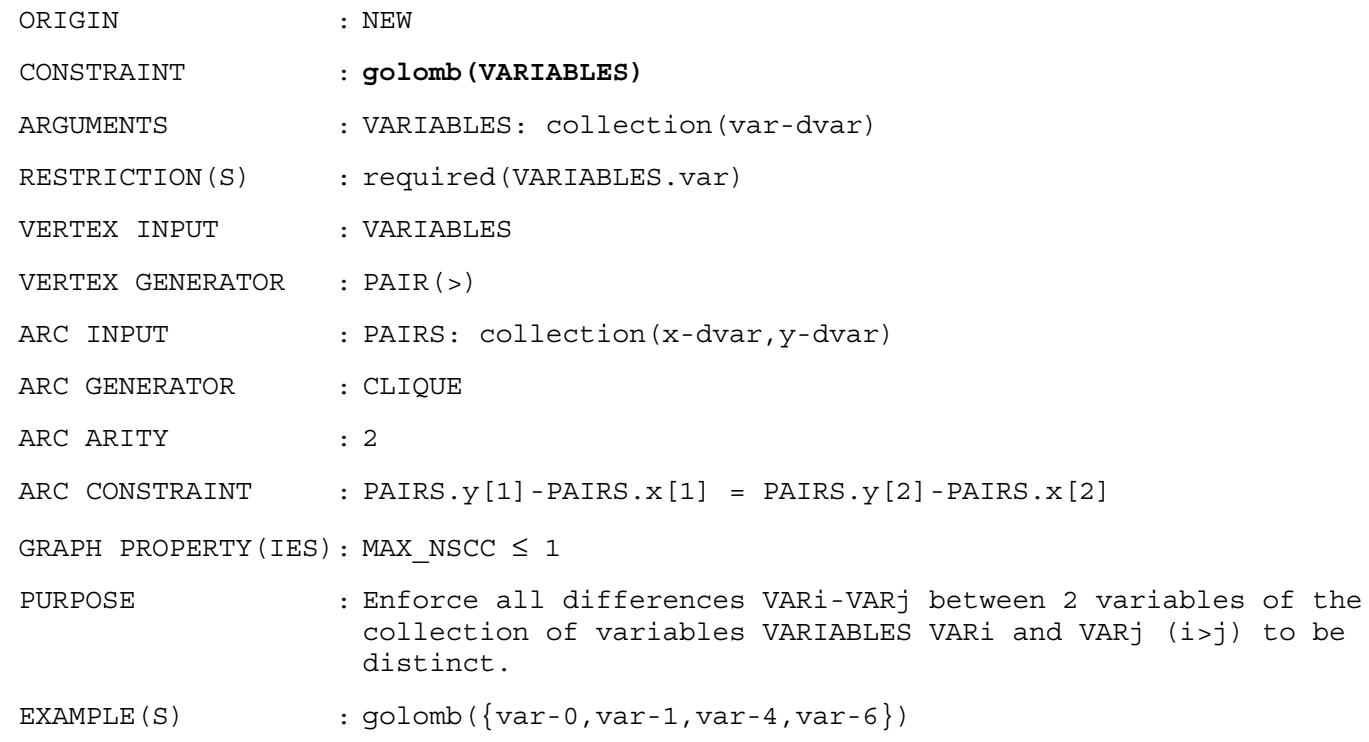

EXAMPLE $(\mathrm{S})$

: golomb ( $\{\operatorname{var}-0, \operatorname{var}-1, \operatorname{var}-4, \operatorname{var}-6\})$

In the next figure, we give the initial graph (A) and show a graphical interpretation (B) of the solution given in the example in term of a graph. Each vertex is a variable, while each arc is a difference between 2 variables. One can see that these differences are all distinct.

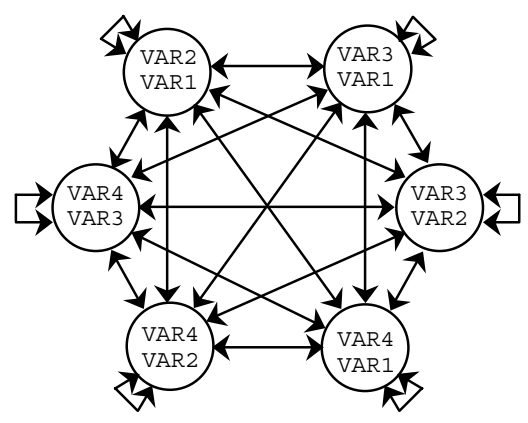

(A)

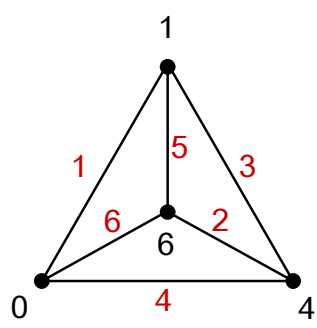

(B)

Figure 13. Initial graph and representation of the solution $0,1,4,6$.

COMMENT

USAGE

: When applied on the collection VAR1, VAR2, VAR3, VAR4, the vertices generator PAIR (>) generates the following vertices in this order: (VAR2, VAR1)

(VAR3, VAR1) (VAR3, VAR2),

(VAR4, VAR1) (VAR4, VAR2), (VAR4, VAR3).

Note that we use a binary arc constraint between 2 vertices and that this binary constraint involves 4 variables.

This constraint refers to the Golomb ruler problem. We take the definition from [SHEARER 96]:

"A Golomb ruler is a set of integers (marks) a(1)< $<<a(k)$ such that all the differences $a(i)-a(j)(i>j)$ are distinct".

REMARK

: At a first glance, one could think that, because it looks so similar to the alldifferent constraint, we would have a perfect polynomial test procedure and also a perfect polynomial pruning procedure. However this is not true since you retrieve the same variable in different vertices of the graph. This will lead to the fact that you have incompatible arcs in the bipartite graph (the 2 classes of vertices correspond to the pair of variables and to the fact that the difference between 2 pairs of variables take a specific value). However you can still use the same algorithms as for the alldifferent constraint, but this will not lead to perfect pruning.

Different constraints models for the Golomb ruler problem were presented in [SMITH, STERGIOU \& WALSH 99]. 


\subsection{Value partitioning constraints}

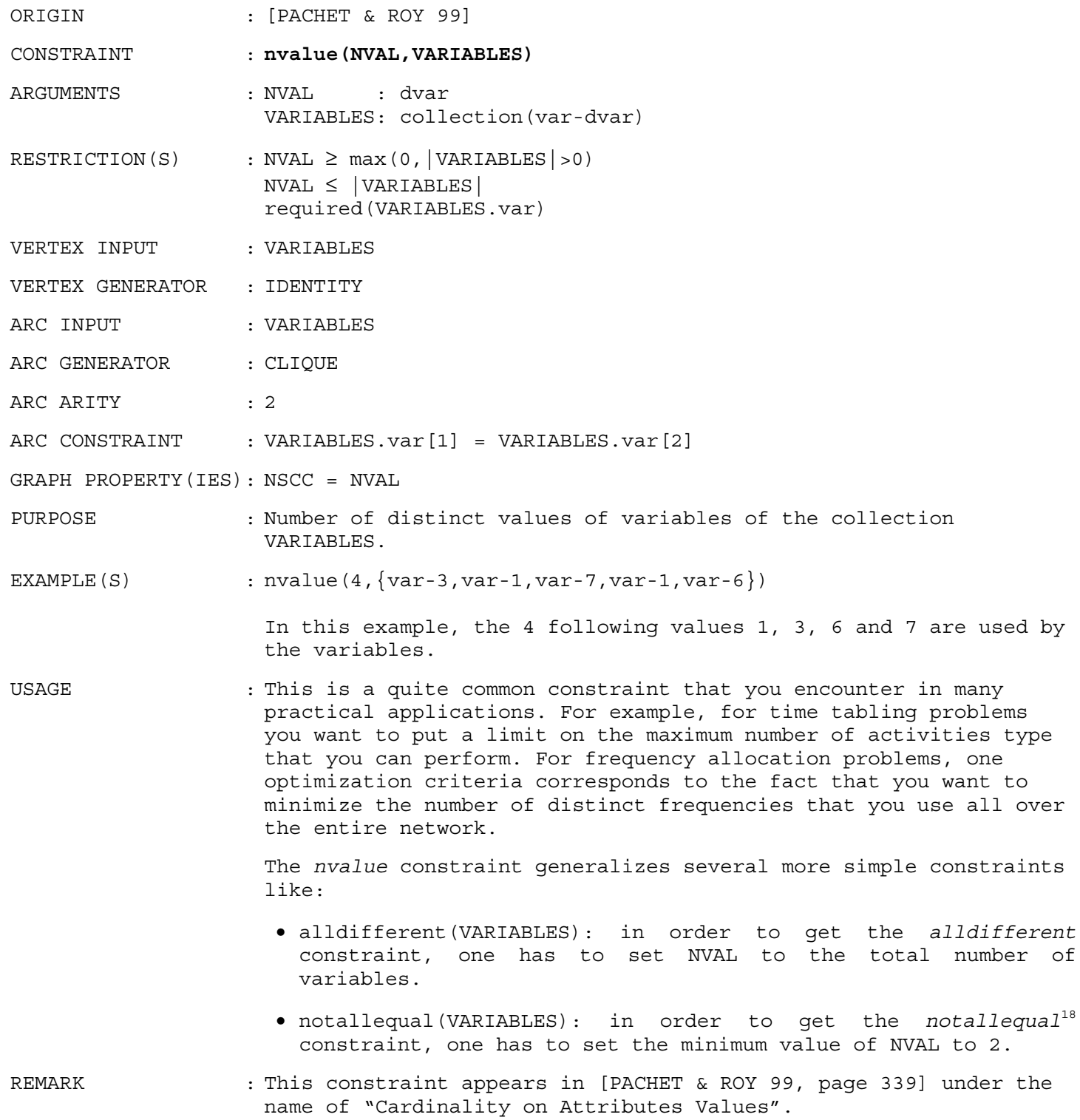

USAGE

: This is a quite common constraint that you encounter in many practical applications. For example, for time tabling problems you want to put a limit on the maximum number of activities type that you can perform. For frequency allocation problems, one optimization criteria corresponds to the fact that you want to minimize the number of distinct frequencies that you use all over the entire network.

The nvalue constraint generalizes several more simple constraints like:

- alldifferent(VARIABLES): in order to get the alldifferent constraint, one has to set NVAL to the total number of variables.

- notallequal (VARIABLES): in order to get the notallequal ${ }^{18}$ constraint, one has to set the minimum value of NVAL to 2.

REMARK

: This constraint appears in [PACHET \& ROY 99, page 339] under the name of "Cardinality on Attributes Values".

${ }^{18}$ The notallequal(VARIABLES) constraint holds if all variables are not all equal. 


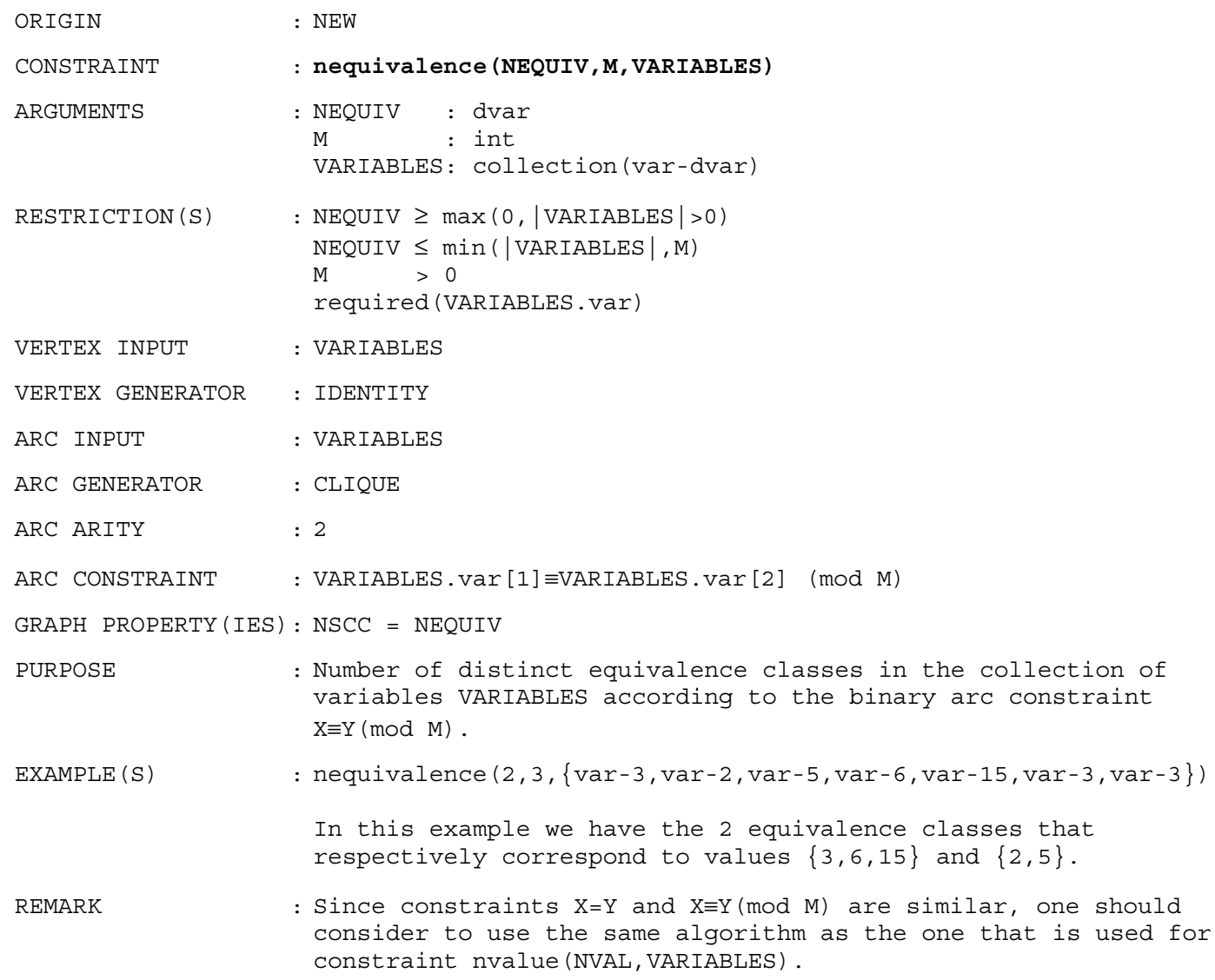

REMARK

: Since constraints $\mathrm{X}=\mathrm{Y}$ and $\mathrm{X} \equiv \mathrm{Y}(\bmod \mathrm{M})$ are similar, one should consider to use the same algorithm as the one that is used for constraint nvalue (NVAL, VARIABLES). 


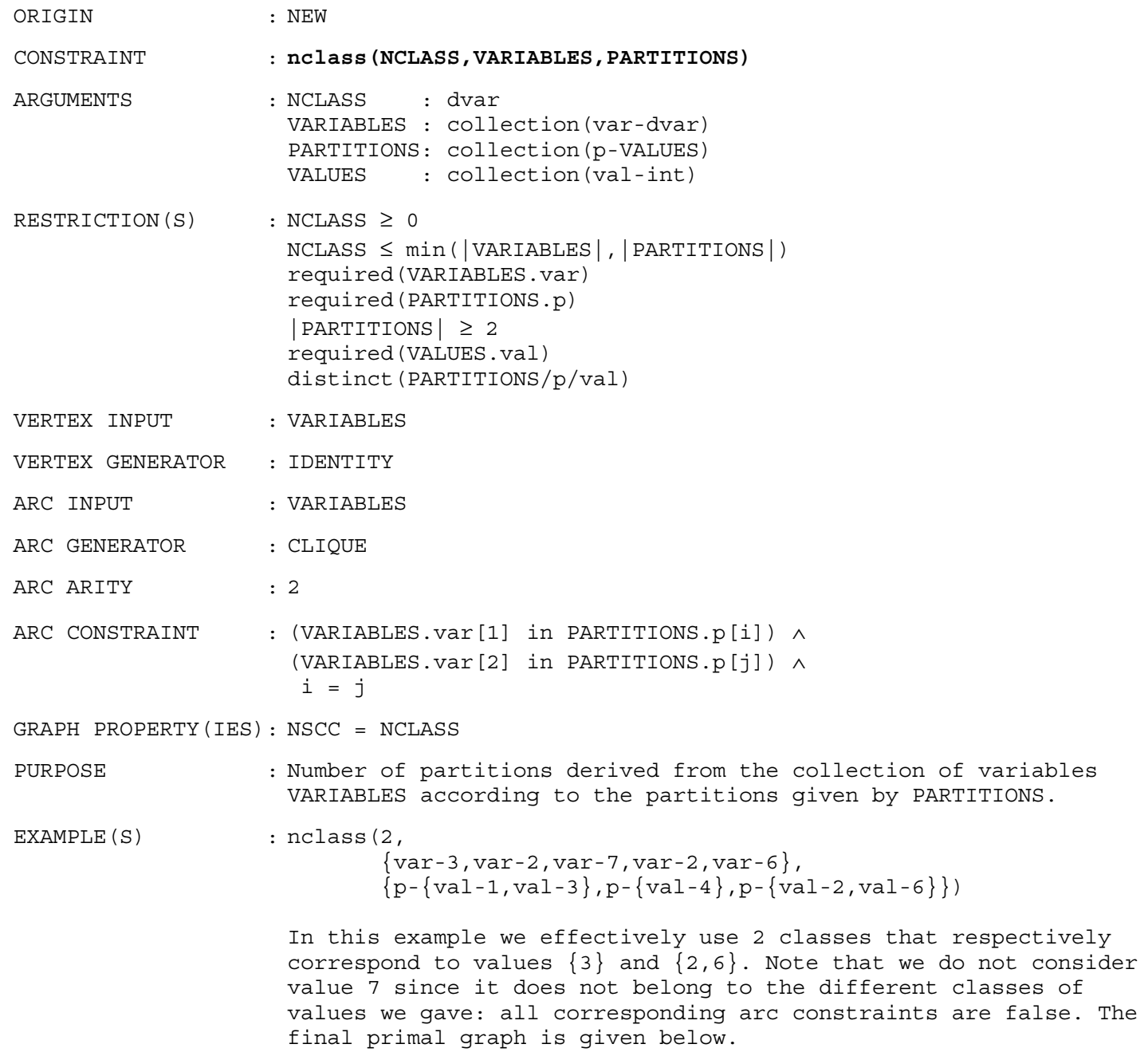

PURPOSE

: Number of partitions derived from the collection of variables VARIABLES according to the partitions given by PARTITIONS.

$\operatorname{EXAMPLE}(\mathrm{S})$

: nclass $(2$,

$$
\begin{aligned}
& \{\operatorname{var}-3, \operatorname{var}-2, \operatorname{var}-7, \operatorname{var}-2, \operatorname{var}-6\}, \\
& \{p-\{\operatorname{val}-1, \operatorname{val}-3\}, p-\{\operatorname{val}-4\}, p-\{\operatorname{val}-2, \operatorname{val}-6\}\})
\end{aligned}
$$

In this example we effectively use 2 classes that respectively correspond to values $\{3\}$ and $\{2,6\}$. Note that we do not consider value 7 since it does not belong to the different classes of values we gave: all corresponding arc constraints are false. The final primal graph is given below.

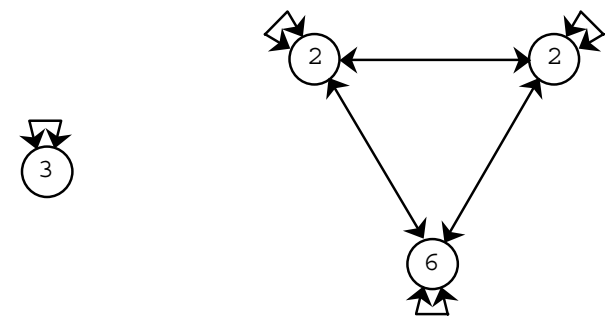

Figure 14. Final primal graph with one strongly connected component for each class of values. 


\subsection{Sliding sequence constraints}

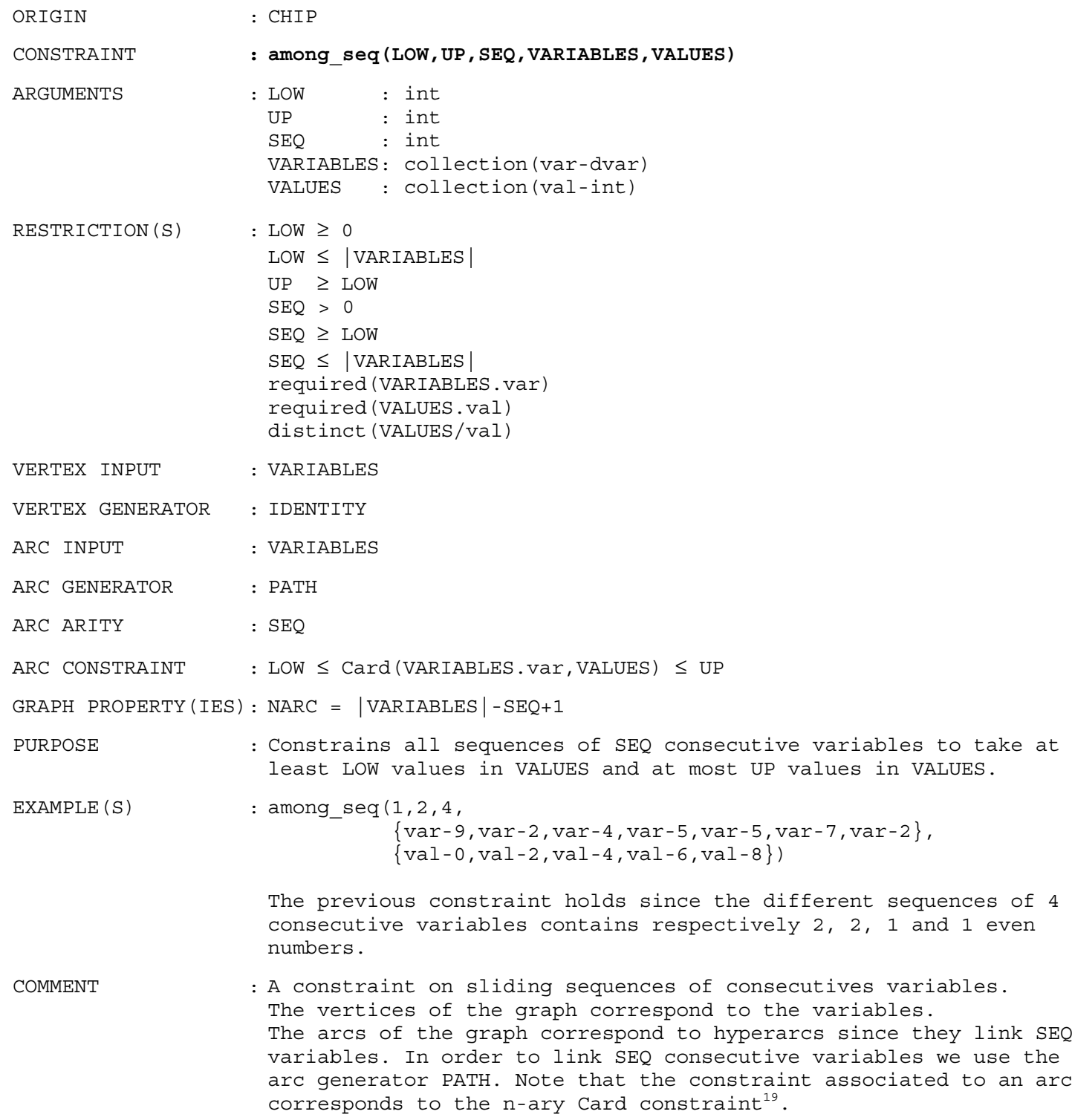

COMMENT

: A constraint on sliding sequences of consecutives variables.

The vertices of the graph correspond to the variables.

The arcs of the graph correspond to hyperarcs since they link SEQ variables. In order to link SEQ consecutive variables we use the arc generator PATH. Note that the constraint associated to an arc corresponds to the n-ary Card constraint ${ }^{19}$.

\footnotetext{
${ }^{19}$ Refers to the Card constraint that was introduced in the paragraph 2.2.1. . It enforces the number of variables that take some specific values to be between 2 given limits.
} 


\begin{tabular}{|c|c|}
\hline ORIGIN & : CHIP \\
\hline CONSTRAINT & : sliding_sum (LOW, UP, SEQ, VARIABLES) \\
\hline ARGUMENTS & $\begin{array}{ll}\text { : LOW } & \text { : int } \\
\text { UP } & \text { : int } \\
\text { SEQ } & \text { : int } \\
\text { VARIABLES }: & \text { collection (var-dvar) }\end{array}$ \\
\hline RESTRICTION (S) & $\begin{array}{l}\mathrm{UP} \geq \mathrm{LOW} \\
\mathrm{SEQ}>0 \\
\mathrm{SEQ} \leq \mid \text { VARIABLES } \\
\text { required (VARIABLES.Var) }\end{array}$ \\
\hline VERTEX INPUT & : VARIABLES \\
\hline VERTEX GENERATOR & : IDENTITY \\
\hline ARC INPUT & : VARIABLES \\
\hline ARC GENERATOR & : PATH \\
\hline ARC ARITY & : SEQ \\
\hline ARC CONSTRAINT & $:$ LOW $\leq$ sum(VARIABLES.var) $\leq \mathrm{UP}$ \\
\hline GRAPH PROPERTIE (S) & $: \mathrm{NARC}=\mid$ VARIABLES $\mid-\mathrm{SEQ}+1$ \\
\hline PURPOSE & $\begin{array}{l}\text { Constrains all sequences of SEQ consecutive variables so that the } \\
\text { sum of the variables is between LOW and UP. }\end{array}$ \\
\hline EXAMPLE (S) & : sliding_sum $(3,7,4,\{\operatorname{var}-1, \operatorname{var}-4, \operatorname{var}-2, \operatorname{var}-0, \operatorname{var}-0, \operatorname{var}-3, \operatorname{var}-4\})$ \\
\hline & $\begin{array}{l}\text { The previous example considers all sliding sequences of } 4 \\
\text { consecutive variables and constraints the sum to be between } 3 \text { and } \\
\text { 7. The constraint holds since the sum associated to the different } \\
\text { sequences are respectively } 7,6,5 \text { and } 7 \text {. }\end{array}$ \\
\hline COMMENT & $\begin{array}{l}\text { : Uses the predefined constraint "LOW } \leq \text { sum(l) } \leq \text { UP" that is } \\
\text { available for any arity. }\end{array}$ \\
\hline
\end{tabular}




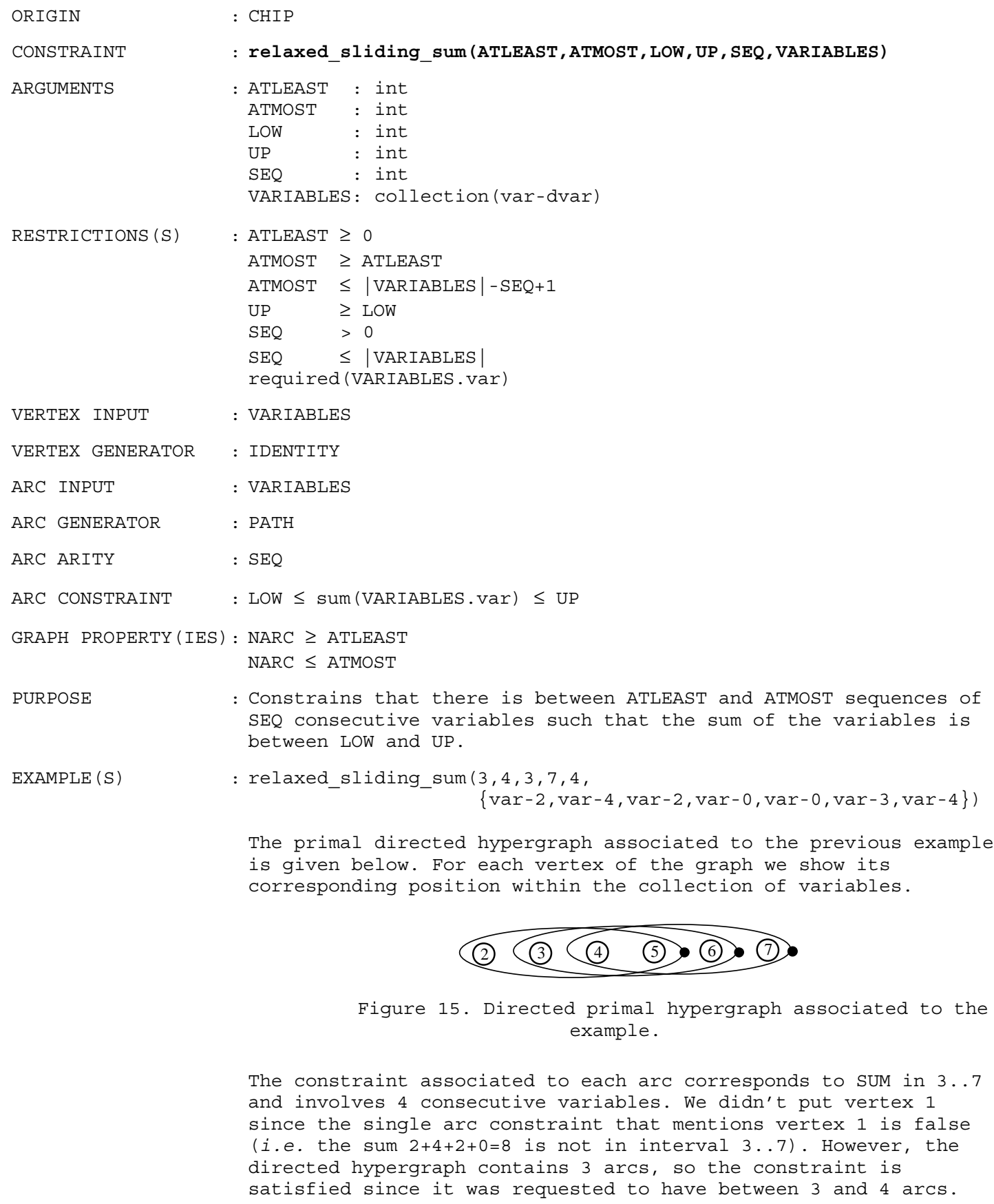

PURPOSE

: Constrains that there is between ATLEAST and ATMOST sequences of SEQ Consecutive variables such that the sum of the variables is between LOW and UP.

$\operatorname{EXAMPLE}(\mathrm{S})$

: relaxed_sliding_sum $(3,4,3,7,4$,

$\{\operatorname{var}-2, \operatorname{var}-4, \operatorname{var}-2, \operatorname{var}-0, \operatorname{var}-0, \operatorname{var}-3, \operatorname{var}-4\})$

The primal directed hypergraph associated to the previous example is given below. For each vertex of the graph we show its corresponding position within the collection of variables.

Figure 15. Directed primal hypergraph associated to the example.

The constraint associated to each arc corresponds to SUM in 3.7 and involves 4 consecutive variables. We didn't put vertex 1 since the single arc constraint that mentions vertex 1 is false (i.e. the sum $2+4+2+0=8$ is not in interval 3..7). However, the directed hypergraph contains 3 arcs, so the constraint is satisfied since it was requested to have between 3 and 4 arcs. 


\subsection{Timetabling constraints}

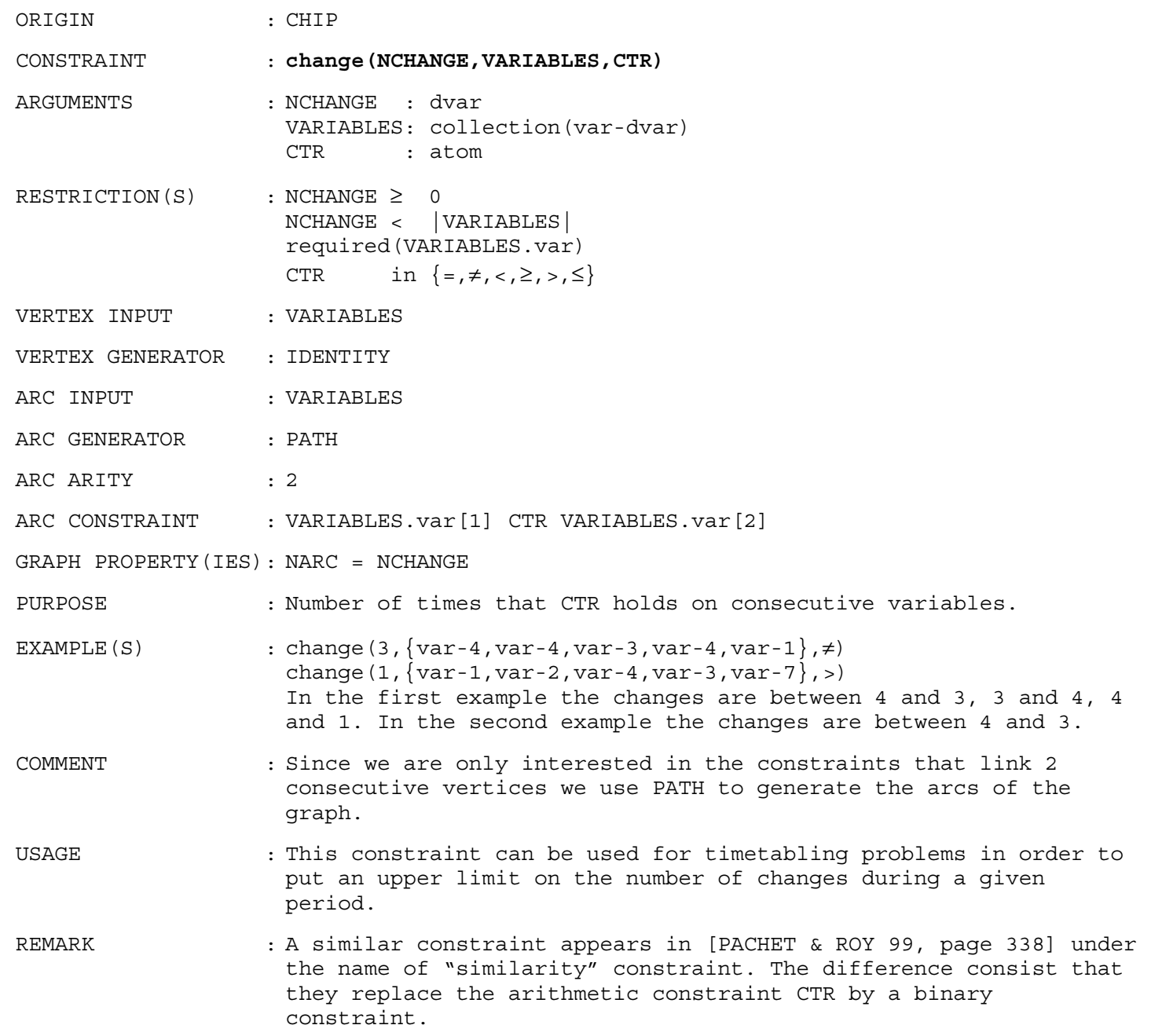




\begin{tabular}{|c|c|}
\hline ORIGIN & : NEW \\
\hline CONSTRAINT & : cyclic_change (NCHANGE, CYCLE_LENGTH, VARIABLES) \\
\hline ARGUMENTS & $\begin{array}{l}\text { NCHANGE } \quad \text { dvar } \\
\text { CYCLE_LENGTH : int } \\
\text { VARIABLES : collection (var-dvar) }\end{array}$ \\
\hline RESTRICTION (S) & $\begin{array}{ll}\text { NCHANGE } & \geq 0 \\
\text { NCHANGE } & <\mid \text { VARIABLES } \mid \\
\text { CYCLE_LENGTH }>0 \\
\text { required (VARIABLES.var) }\end{array}$ \\
\hline VERTEX INPUT & : VARIABLES \\
\hline VERTEX GENERATOR & : IDENTITY \\
\hline ARC INPUT & : VARIABLES \\
\hline ARC GENERATOR & : PATH \\
\hline ARC ARITY & $: 2$ \\
\hline ARC CONSTRAINT & $:(($ VARIABLES.var $[1]+1)$ mod CYCLE_LENGTH $) \neq$ VARIABLES.var [2] \\
\hline GRAPH PROPERTY (IES) : & $:$ NARC $=$ NCHANGE \\
\hline PURPOSE & $\begin{array}{l}\text { Number of times that constraint }((X+1) \text { mod CYCLE_LENGTH }) \neq Y \text { holds. } \\
\text { ( } X \text { and } Y \text { correspond to consecutive variables of VARIABLES) }\end{array}$ \\
\hline EXAMPLE (S) & : cyclic_change $(2,4,\{\operatorname{var}-3, \operatorname{var}-0, \operatorname{var}-2, \operatorname{var}-3, \operatorname{var}-1\})$ \\
\hline & $\begin{array}{l}\text { In this example we have the } 2 \text { following "cyclic" changes: } \\
\text { - a first change between } 0 \text { and } 2 \text {, } \\
\text { - a second change between } 3 \text { and } 1 \text {. } \\
\text { However, the sequence " } 0 \text { " is not a change since (3+1) mod } 4 \text { is } \\
\text { equal to } 0 .\end{array}$ \\
\hline USAGE & $\begin{array}{l}\text { This constraint may be used for personnel cyclic timetabling } \\
\text { problems where each person has to work according to cycles; } \\
\text { however, because in reality you have some perturbation it is not } \\
\text { reasonable to ask for perfect cyclic solutions. One alternative } \\
\text { would be to use the cycle_change constraint and to ask for } \\
\text { solutions where one tries to minimize the number of cycle breaks } \\
\text { (i.e. the variable NCHANGE). }\end{array}$ \\
\hline
\end{tabular}




\begin{tabular}{|c|c|}
\hline ORIGIN & : NEW \\
\hline CONSTRAINT & : cYclic_change_joker(NCHANGE, CYCLE_LENGTH,VARIABLES) \\
\hline ARGUMENTS & $\begin{array}{l}\text { : NCHANGE : dvar } \\
\text { CYCLE_LENGTH : int } \\
\text { VARIABLES : collection (var-dvar) }\end{array}$ \\
\hline RESTRICTION (S) & $\begin{array}{l}\text { NCHANGE } \quad \geq 0 \\
\text { NCHANGE } \quad<\mid \text { VARIABLES } \\
\text { required (VARIABLES.var) } \\
\text { CYCLE_LENGTH }>0\end{array}$ \\
\hline VERTEX INPUT & : VARIABLES \\
\hline VERTEX GENERATOR & : IDENTITY \\
\hline ARC INPUT & : VARIABLES \\
\hline ARC GENERATOR & : PATH \\
\hline ARC ARITY & $: 2$ \\
\hline ARC CONSTRAINT & $\begin{array}{l}:((\text { VARIABLES.var }[1]+1) \bmod \text { CYCLE_LENGTH }) \neq \text { VARIABLES.var [2] } \\
\text { VARIABLES.var }[1]<\text { CYCLE_LENGTH } \\
\text { VARIABLES.var }[2]<\text { CYCLE_LENGTH }\end{array}$ \\
\hline \multicolumn{2}{|c|}{ GRAPH PROPERTY (IES) $:$ NARC = NCHANGE } \\
\hline PURPOSE & $\begin{array}{l}\text { Number of times that the following constraint holds: } \\
((X+1) \text { mod CYCLE_LENGTH }) \neq Y \wedge X<C Y C L E \text { LENGTH } \wedge \text { Y C CYCLE_LENGTH. } \\
(X \text { and } Y \text { correspond to consecutive variables of VARIABLES })\end{array}$ \\
\hline \multirow[t]{2}{*}{ EXAMPLE (S) } & $\begin{aligned}: \text { cyclic_change_joker }(2,4, & \{\operatorname{var}-3, \operatorname{var}-0, \operatorname{var}-2, \\
& \operatorname{var}-4, \operatorname{var}-4, \operatorname{var}-4 \\
& \operatorname{var}-3, \operatorname{var}-1, \operatorname{var}-4\})\end{aligned}$ \\
\hline & $\begin{array}{l}\text { In this example we have the } 2 \text { following cyclic changes: } \\
\text { - a first change between } 0 \text { and } 2 \text {, } \\
\text { - a second change between } 3 \text { and } 1 \text {. } \\
\text { But when the "joker" value } 4 \text { is involved, there is no change; } \\
\text { this is why no change is counted between } 2 \text { and } 4 \text {, between } 4 \text { and } \\
4 \text {, between } 1 \text { and } 4 .\end{array}$ \\
\hline COMMENT & $\begin{array}{l}\text { : The "joker values" are the values that are greater or equal than } \\
\text { CYCLE_LENGTH. We do not count any change for the binary arc } \\
\text { constraints involving at least one variable that takes a joker } \\
\text { value. }\end{array}$ \\
\hline USAGE & $\begin{array}{l}\text { This constraint may be used in the same context as the } \\
\text { cycle change constraint with the additional interpretation: in } \\
\text { our example codes o to } 3 \text { correspond to different type of } \\
\text { activities (i.e. working the morning, the afternoon and the } \\
\text { night) and code } 4 \text { represents the fact that you don't work at all } \\
\text { during a given day. We want to specify that there is no change } \\
\text { when you go on holiday, when you are in holiday and when you come } \\
\text { back from holiday. In all other situations you should still work } \\
\text { in a cyclic way. }\end{array}$ \\
\hline
\end{tabular}




\begin{tabular}{|c|c|}
\hline ORIGIN & : NEW \\
\hline CONSTRAINT & : smooth (NCHANGE, TOLERANCE, VARIABLES) \\
\hline ARGUMENTS & $\begin{array}{l}\text { NCHANGE : dvar } \\
\text { TOLERANCE: int } \\
\text { VARIABLES: collection (var-dvar) }\end{array}$ \\
\hline RESTRICTION (S) & $\begin{array}{l}: \text { NCHANGE } \geq 0 \\
\text { NCHANGE }<\mid \text { VARIABLES } \\
\text { TOLERANCE } \geq 0 \\
\text { required (VARIABLES.var) }\end{array}$ \\
\hline VERTEX INPUT & : VARIABLES \\
\hline VERTEX GENERATOR & : IDENTITY \\
\hline ARC INPUT & : VARIABLES \\
\hline ARC GENERATOR & : PATH \\
\hline ARC ARITY & $: 2$ \\
\hline $\begin{array}{l}\text { ARC CONSTRAINT } \\
\text { GRAPH PROPERTY (IE }\end{array}$ & $\begin{array}{l}: \mid \text { VARIABLES.var }[1]-\text { VARIABLES.var }[2] \mid>\text { TOLERANCE } \\
: \text { NARC = NCHANGE }\end{array}$ \\
\hline PURPOSE & $\begin{array}{l}\text { : Number of times that }|\mathrm{X}-\mathrm{Y}|>\text { TOLERANCE holds. } \\
(\mathrm{X} \text { and } \mathrm{Y} \text { correspond to consecutive variables of VARIABLES) }\end{array}$ \\
\hline EXAMPLE (S) & $\begin{array}{l}\text { : } \operatorname{smooth}(1,2,\{\operatorname{var}-1, \operatorname{var}-3, \operatorname{var}-4, \operatorname{var}-5, \operatorname{var}-2\}) \\
\text { In the previous example we have one change between values } 5 \text { and } 2 \\
\text { since the difference in absolute value is greater than the } \\
\text { tolerance }(i . e .|5-2|>2) \text {. }\end{array}$ \\
\hline USAGE & $\begin{array}{l}\text { - This constraint can be useful for the following problems: } \\
\text { - Assume that VARIABLES corresponds to the number of people that } \\
\text { work on consecutive weeks. One may not normally increase or } \\
\text { decrease too drastically the number of people from one week to } \\
\text { the next week. With the smooth constraint you could put a limit } \\
\text { on the number of drastic changes. } \\
\text { - Assume you have to produce a set of orders, each order having a } \\
\text { specific attribute. You want to generate the orders in such a } \\
\text { way that there is not a too big difference between the values } \\
\text { of the attributes of } 2 \text { consecutives orders. If you can't } \\
\text { achieve this on } 2 \text { given specific orders, this would imply a } \\
\text { set-up or a cost. You would like to minimize this kind of } \\
\text { break. }\end{array}$ \\
\hline
\end{tabular}




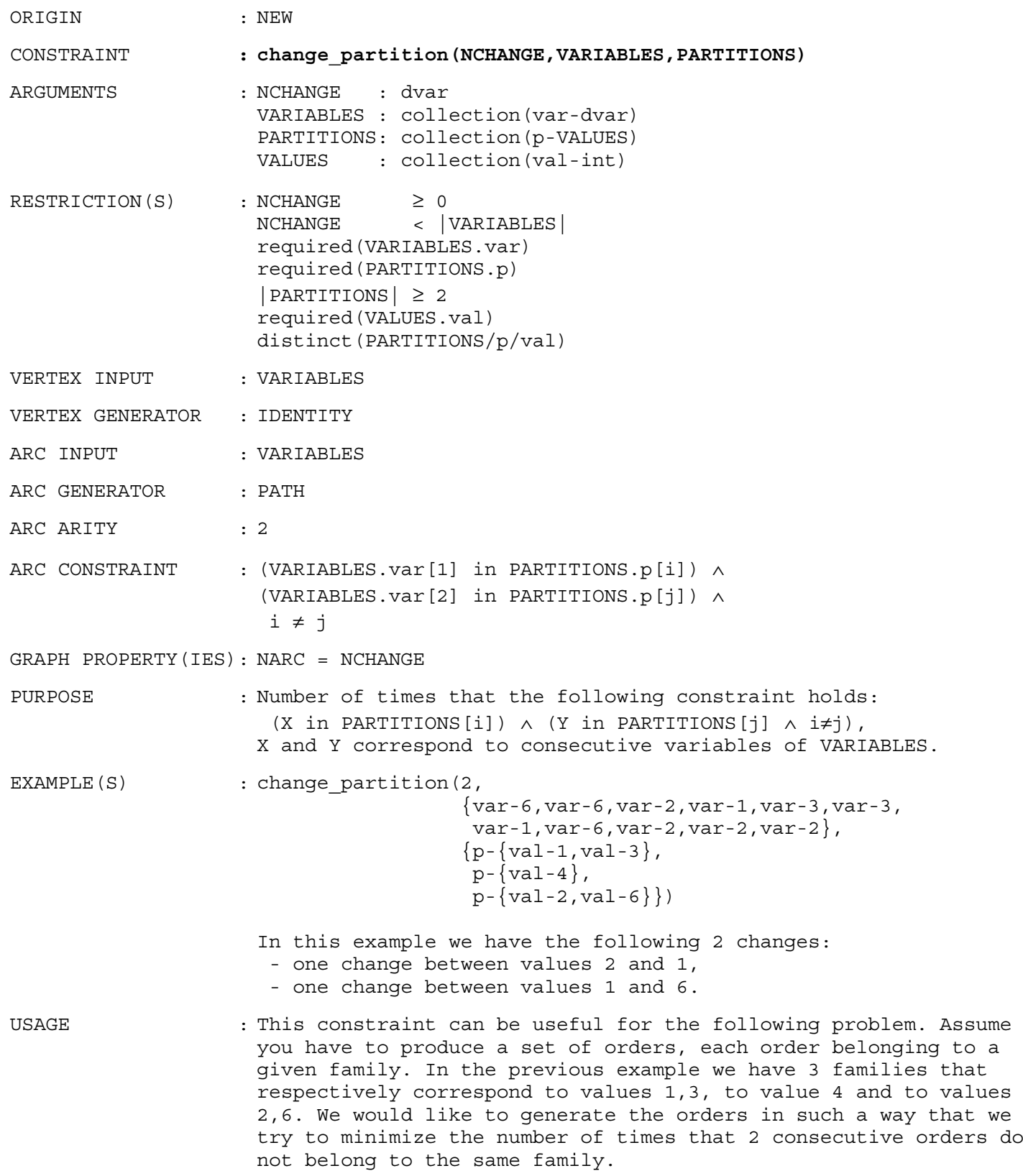

In this example we have the following 2 changes:

- one change between values 2 and 1 ,

- one change between values 1 and 6 .

USAGE

: This constraint can be useful for the following problem. Assume you have to produce a set of orders, each order belonging to a given family. In the previous example we have 3 families that respectively correspond to values 1,3 , to value 4 and to values 2,6 . We would like to generate the orders in such a way that we try to minimize the number of times that 2 consecutive orders do not belong to the same family. 


\begin{tabular}{|c|c|}
\hline ORIGIN & : NEW \\
\hline CONSTRAINT & : longest_changes (SIZE, VARIABLES, CTR) \\
\hline ARGUMENTS & $\begin{array}{ll}\text { : SIZE } & \text { dvar } \\
\text { VARIABLES: } & \text { collection (var-dvar) } \\
\text { CTR } \quad \text { : atom }\end{array}$ \\
\hline RESTRICTION (S) & $\begin{array}{l}\text { SIZE } \geq 0 \\
\text { required (VARIABLES. var) } \\
\text { CTR in }\{=, \neq,<, \geq,>, \leq\}\end{array}$ \\
\hline VERTEX INPUT & : VARIABLES \\
\hline VERTEX GENERATOR & : IDENTITY \\
\hline ARC INPUT & : VARIABLES \\
\hline ARC GENERATOR & : PATH \\
\hline ARC ARITY & $: 2$ \\
\hline ARC CONSTRAINT & : VARIABLES.var[1] CTR VARIABLES.var[2] \\
\hline \multicolumn{2}{|c|}{ GRAPH PROPERTY (IES) : MAX_NCC $=$ SIZE } \\
\hline PURPOSE & $\begin{array}{l}\text { Maximum number of consecutive variables for which constraint CTR } \\
\text { holds in an uninterrupted way; we have a change when CTR holds on } \\
2 \text { consecutive variables. }\end{array}$ \\
\hline \multirow[t]{2}{*}{ EXAMPLE (S) } & $\begin{array}{r}\text { : longest_changes }(4,\{\operatorname{var}-8, \operatorname{var}-8, \operatorname{var}-3, \operatorname{var}-4, \operatorname{var}-1, \\
\operatorname{var}-1, \operatorname{var}-5, \operatorname{var}-5, \operatorname{var}-2\}, \neq)\end{array}$ \\
\hline & $\begin{array}{l}\text { On this example the longest period of uninterrupted changes } \\
\text { corresponds to the sequence } 8,3,4,1 \text {, which involves } 4 \\
\text { consecutives variables. }\end{array}$ \\
\hline COMMENT & $\begin{array}{l}\text { : In order to specify the constraint, we use MAX_NCC, which is the } \\
\text { number of vertices of the largest connected component. In our } \\
\text { case, since the initial graph corresponds to a path, this will be } \\
\text { the length of the longest path in the final primal graph. }\end{array}$ \\
\hline
\end{tabular}




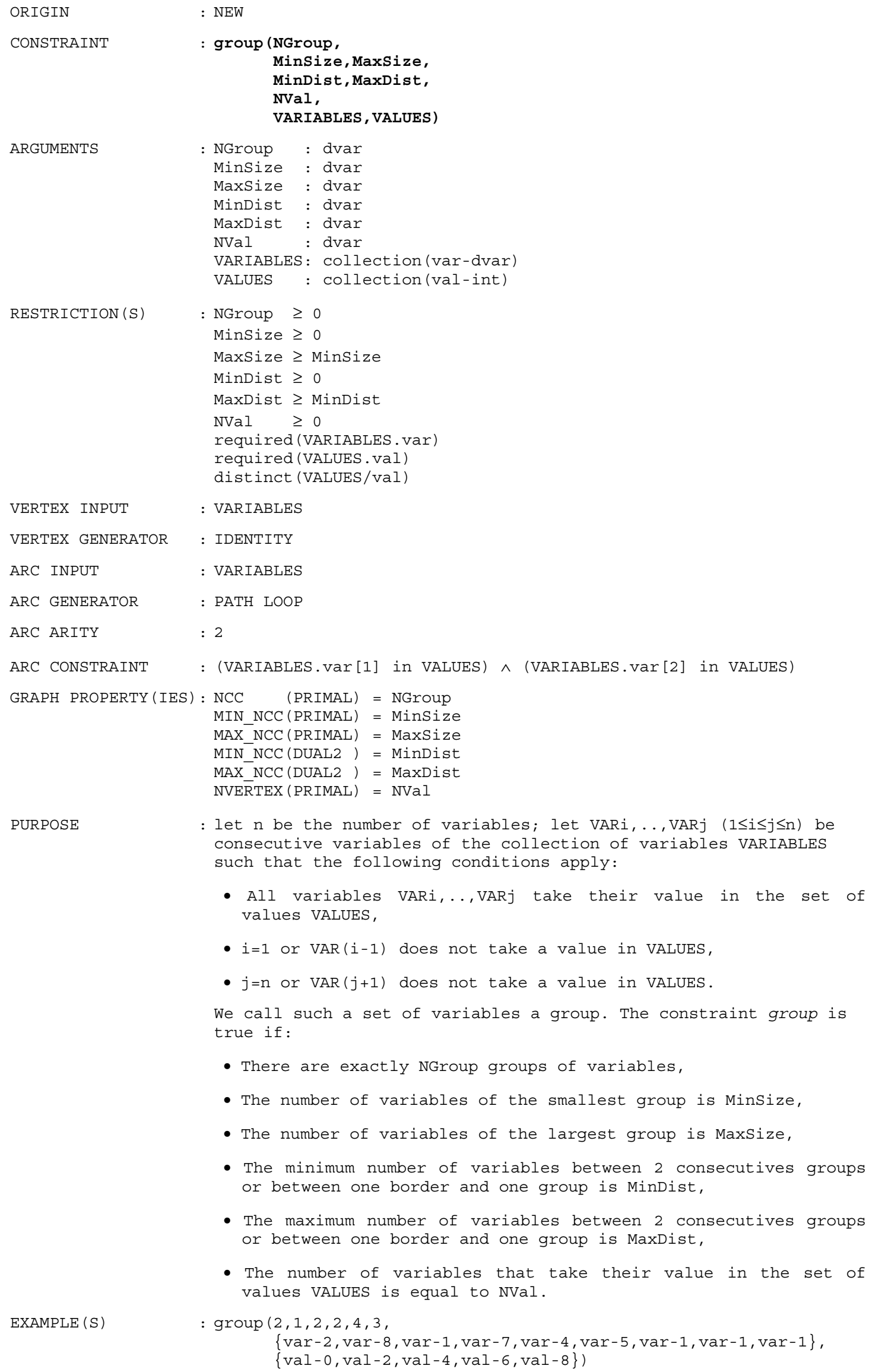
consecutive variables of the collection of variables VARIABLES such that the following conditions apply:

- All variables VARi,..,VARj take their value in the set of values VALUES,

- $i=1$ or $\operatorname{VAR}(i-1)$ does not take a value in VALUES,

- $j=n$ or $\operatorname{VAR}(j+1)$ does not take a value in VALUES.

We call such a set of variables a group. The constraint group is true if:

- There are exactly NGroup groups of variables,

- The number of variables of the smallest group is Minsize,

- The number of variables of the largest group is MaxSize,

- The minimum number of variables between 2 consecutives groups or between one border and one group is MinDist,

- The maximum number of variables between 2 consecutives groups or between one border and one group is MaxDist,

- The number of variables that take their value in the set of values VALUES is equal to NVal.

EXAMPLE (S)

: group $(2,1,2,2,4,3$,

$\{\operatorname{var}-2, \operatorname{var}-8, \operatorname{var}-1, \operatorname{var}-7, \operatorname{var}-4, \operatorname{var}-5, \operatorname{var}-1, \operatorname{var}-1, \operatorname{var}-1\}$, $\{\mathrm{val}-0, \mathrm{val}-2, \mathrm{val}-4, \mathrm{val}-6, \mathrm{val}-8\}$ ) 
The next figure gives the graph that corresponds to the solution of the example. The primal graph is given in dark, while the dual graph of type 2 is shown in gray. For each vertex of the graph we show its corresponding position within the collection. Below each vertex we indicate the value of the corresponding variable.

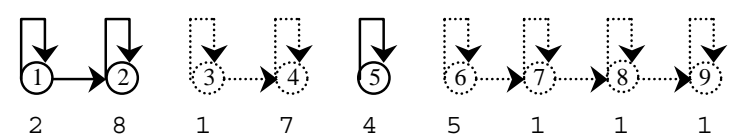

Figure 16. Primal and dual graphs of the group constraint.

Since the primal graph has 2 connected components the number of groups NGroup is 2.

Since the number of vertices in the smallest connected component of the primal graph is 1, Minsize is equal to 1 .

Since the number of vertices in the largest connected component of the primal graph is 2, MaxSize is equal to 2 .

Since the size of the smallest connected component of the dual graph is 2, MinDist is equal to 2 .

Since the size of the largest connected component of the dual graph is 4, MaxDist is equal to 4 .

Since the number of vertices of the primal graph is 3, NVal is equal to 3 .

: In order to generate the initial graph we use:

- The arc generators PATH and LOOP,

- The binary constraint "X in VALUES $\wedge \mathrm{Y}$ in VALUES".

This gives an initial graph of the following form:

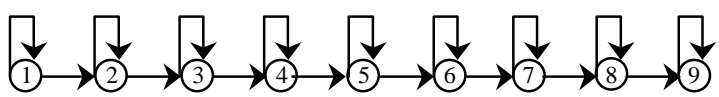

Figure 17. Initial graph of the group constraint.

We use PATH LOOP and the binary constraint ( $\mathrm{X}$ in VALUES) $\wedge$ ( $\mathrm{Y}$ in VALUES) in order to catch the 2 following situations:

- A binary constraint has to be used in order to get the notion of group: consecutive variables that take their value in VALUES.

- If we only use PATH then we lose the groups that are composed from one single variable since the predecessor and the successor arc would be destroyed; this is why we use also the LOOP generator.

: For this constraint we use the possibility to express directly more than one constraint on the characteristics of the graph. For more propagation, it is crucial to keep this in one single constraint, since strong mathematical relations relate all the characteristics of the graph.

This constraint is very similar to the group constraint introduced in CHIP, except that here, the MinDist and MaxDist constraints apply also for the 2 borders: we can not start or end with a group of $n$ consecutive variables that take their values outside VALUES and such that $n$ is less than MinDist or $n$ is greater than MaxDist. 


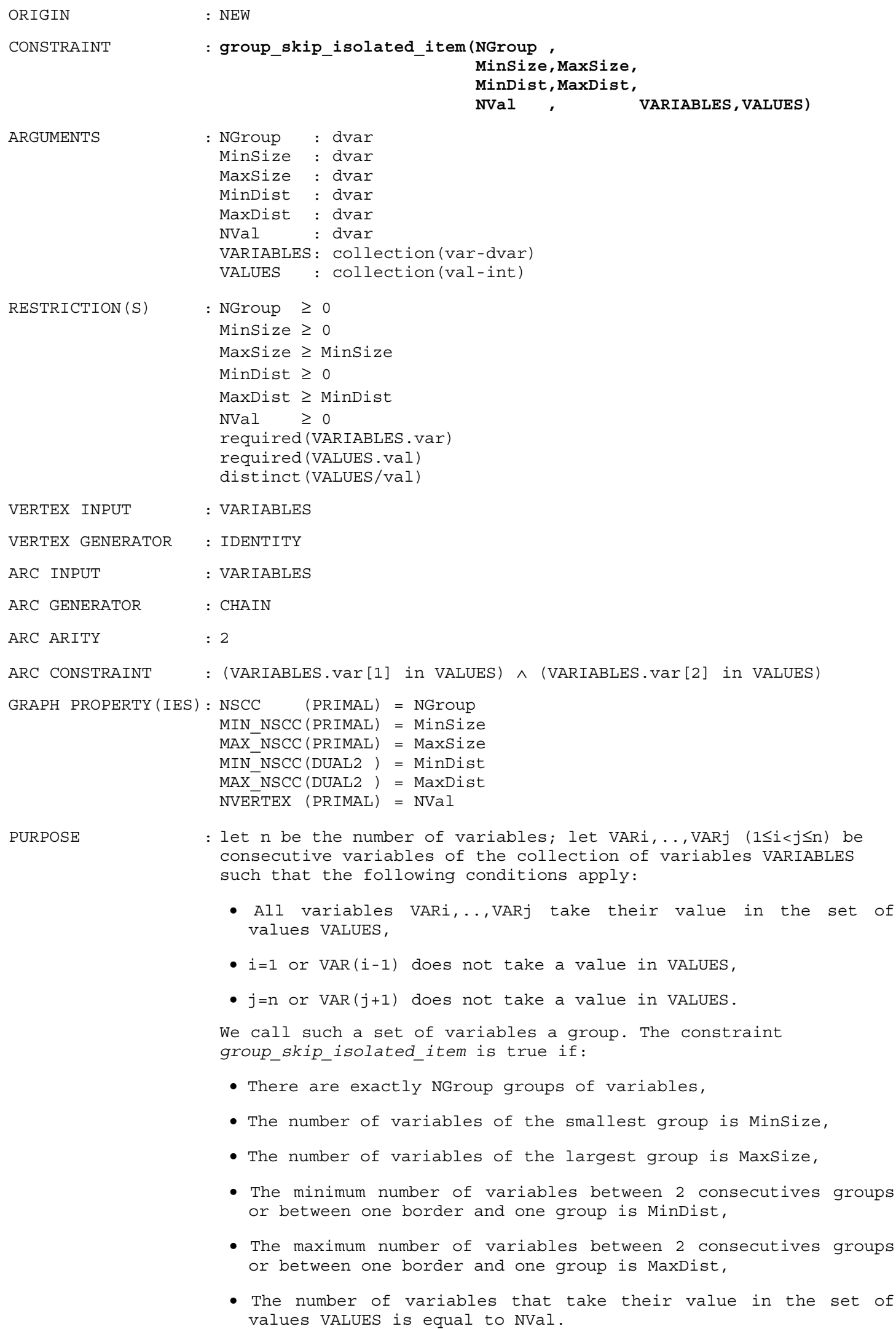

PURPOSE

: let $n$ be the number of variables; let VARi, . VARj $(1 \leq i<j \leq n)$ be consecutive variables of the collection of variables VARIABLES such that the following conditions apply:

- All variables VARi,..,VARj take their value in the set of values VALUES,

- $i=1$ or $\operatorname{VAR}(i-1)$ does not take a value in VALUES,

- $j=n$ or $\operatorname{VAR}(j+1)$ does not take a value in VALUES.

We call such a set of variables a group. The constraint group_skip_isolated_item is true if:

- There are exactly NGroup groups of variables,

- The number of variables of the smallest group is Minsize,

- The number of variables of the largest group is MaxSize,

- The minimum number of variables between 2 consecutives groups or between one border and one group is MinDist,

- The maximum number of variables between 2 consecutives groups or between one border and one group is MaxDist,

- The number of variables that take their value in the set of values VALUES is equal to NVal. 
: group skip isolated item $(1,2,2,7,7,2$,

$\{\operatorname{var}-2, \operatorname{var}-8, \operatorname{var}-1, \operatorname{var}-7, \operatorname{var}-4$,

$\operatorname{var}-5, \operatorname{var}-1, \operatorname{var}-1, \operatorname{var}-1\}$,

$\{\mathrm{val}-0, \mathrm{val}-2, \mathrm{val}-4, \mathrm{val}-6, \mathrm{val}-8\}$

The next figure gives the graph that corresponds to the solution of the example. The primal graph is given in dark, while the dual graph of type 2 is shown in gray. For each vertex of the graph we show its corresponding position within the collection. Below each vertex we indicate the value of the corresponding variable.

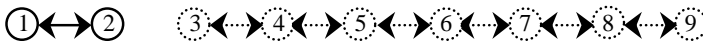

$\begin{array}{lllllllll}2 & 8 & 1 & 7 & 4 & 5 & 1 & 1 & 1\end{array}$

Figure 18. Primal and dual graphs associated to group_skip_isolated_item.

Since we have one strongly connected component in the primal graph the number of groups is 1 .

Since the strongly connected component of the primal graph contains 2 vertices, MinSize and MaxSize are both equal to 2 .

Since we have only one strongly connected component in the dual graph and since it contains 7 vertices, MinDist and MaxDist are both equal to 7 .

Since the number of vertices of the primal graph is 2, NVal is equal to 2 .

: We use chain to generate the initial graph. This generates a graph (with binary constraints) of the following form:

\section{(1) $\leftrightarrow(2) \leftrightarrow(3) \leftrightarrow(4) \leftrightarrow(5) \leftrightarrow(6) \leftrightarrow(2) \leftrightarrow(8) \leftrightarrow(9)$}

Figure 19. Initial graph for group_skip_isolated_item.

We use chain and the "in" elementary constraint

(i.e. $X$ in VALUES $\wedge \mathrm{Y}$ in VALUES) in order to skip the isolated variables that take a value in VALUES that we don't want to count as a group. On the example, this is why value 4 is not counted as a group.

: This constraint can be useful in order to specify rules about how rest days should be allocated to a person during a period of $n$ consecutive days. In this case VALUES are the codes for the rest days (perhaps one single value) and VARIABLES corresponds to the amount of work done during $n$ consecutive days. We can then express a rule like: in a month one should have at least 4 periods of at least 2 rest days; isolated rest days are not counted as rest periods. 


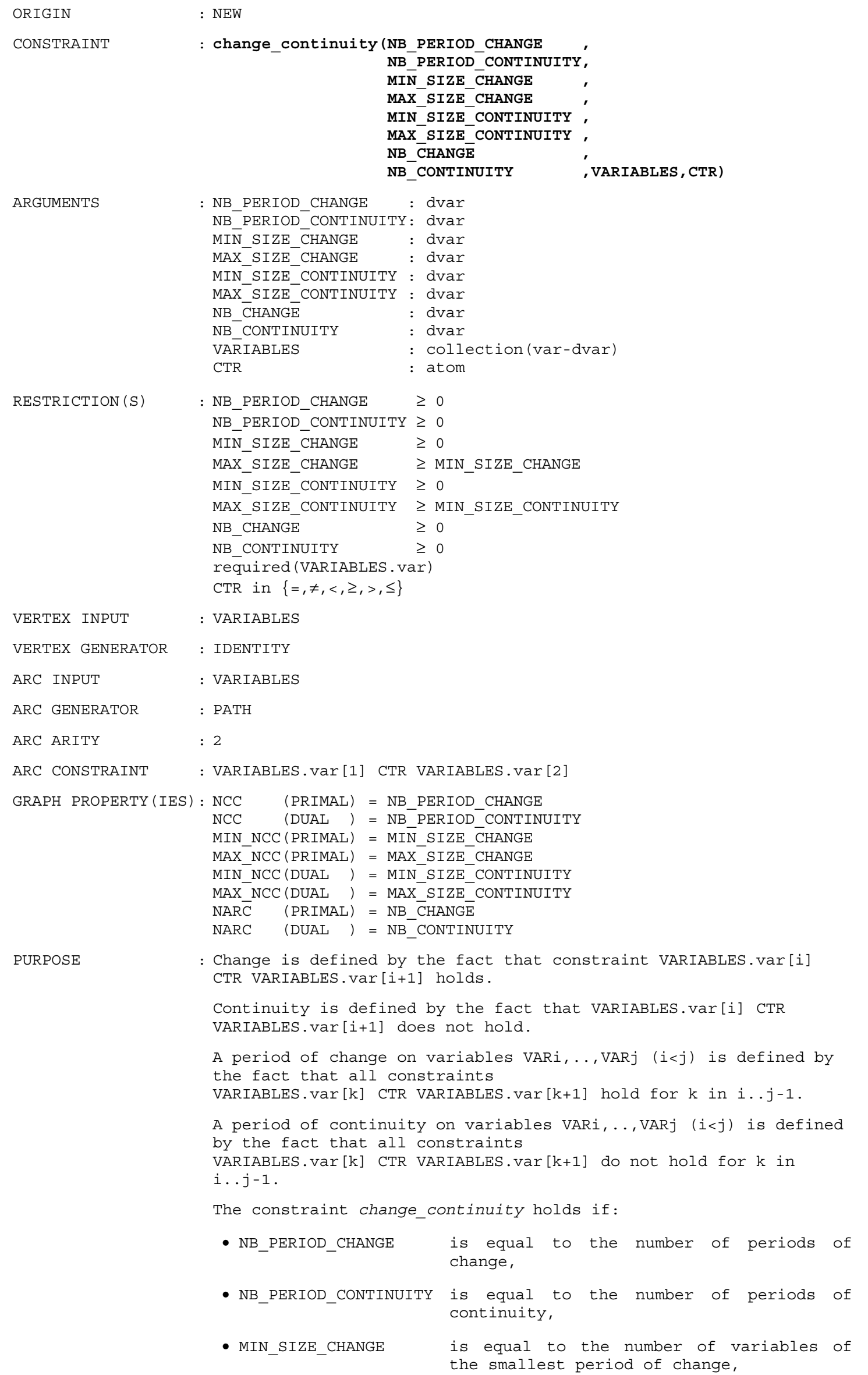


- MAX_SIZE_CHANGE ${ }^{20}$

is equal to the number of variables of the largest period of change,

- MIN_SIZE_CONTINUITY is equal to the number of variables of the smallest period of continuity,

- MAX_SIZE_CONTINUITY is equal to the number of variables of the largest period of continuity,
- NB_CHANGE

- NB_CONTINUITY

EXAMPLE $(S)$ is equal to the total number of changes,

is equal to the total number of continuities.

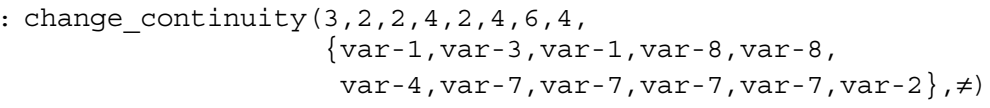

The following schema makes clear the different parameters that are associated to the given example. We place character for representing a change and a blank for a continuity. On top of the solution we represent the different periods of change, while below we show the different periods of continuity.

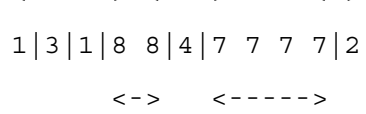

Figure 20. Periods of changes and periods of continuities.

\footnotetext{
${ }^{20}$ If the variables of the collection VARIABLES have to take distinct values between 1 and the total number of variables, we have what is called a permutation. In this case, if we choose the constraint $<$, then MAX_SIZE_CHANGE gives the size of the longest run of the permutation; a run is a maximal increasing contiguous subsequence in the permutation.
} 


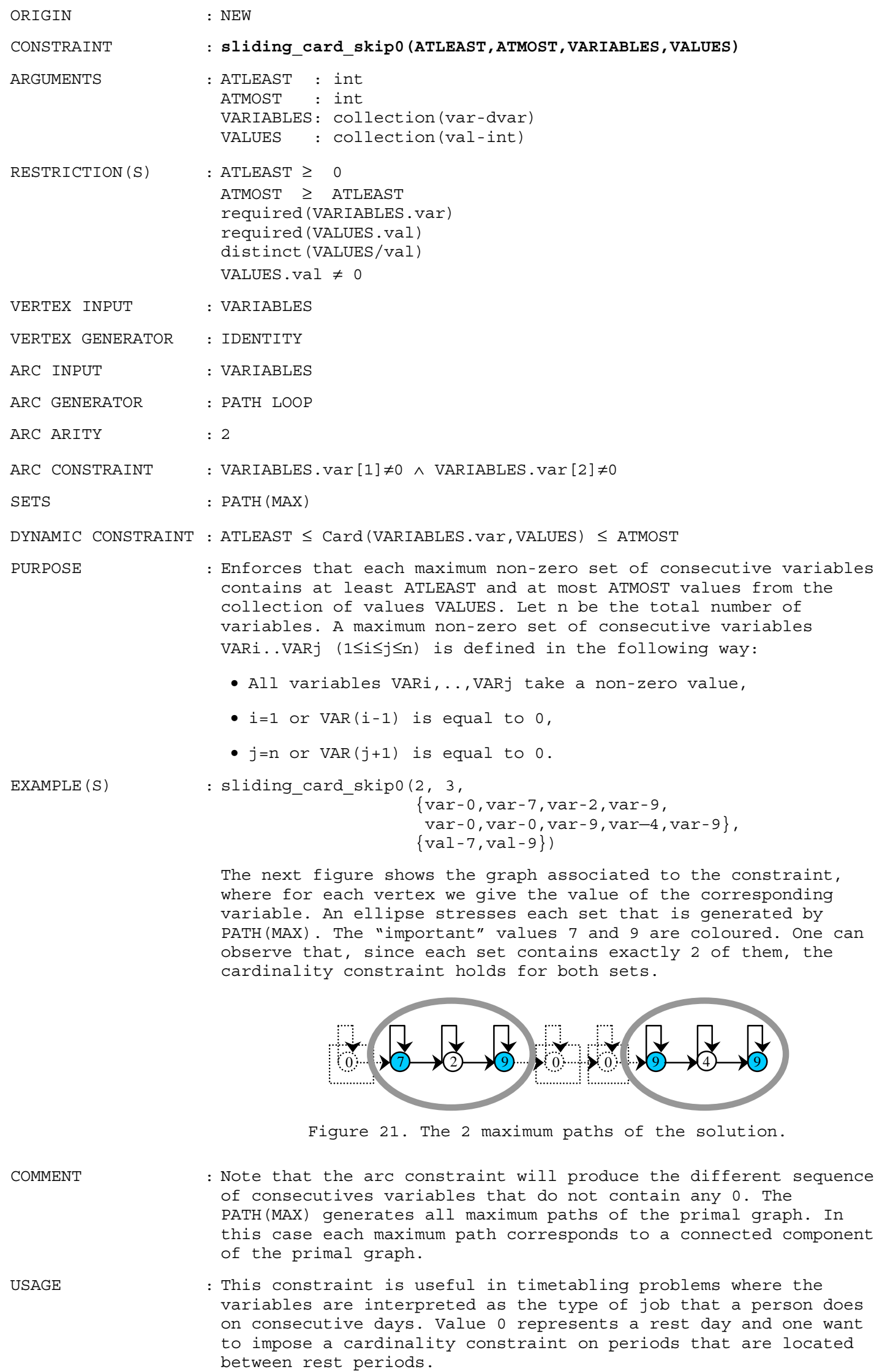
contains at least ATLEAST and at most ATMOST values from the collection of values VALUES. Let $\mathrm{n}$ be the total number of variables. A maximum non-zero set of consecutive variables VARi..VARj $(1 \leq i \leq j \leq n)$ is defined in the following way:

- All variables VARi,..,VARj take a non-zero value,

- $i=1$ or $\operatorname{VAR}(i-1)$ is equal to 0

- $j=n$ or $\operatorname{VAR}(j+1)$ is equal to 0 .

EXAMPLE ( $)$

: sliding_card_skipo $(2,3$,

The next figure shows the graph associated to the constraint where for each vertex we give the value of the corresponding variable. An ellipse stresses each set that is generated by PATH (MAX). The "important" values 7 and 9 are coloured. One can observe that, since each set contains exactly 2 of them, the cardinality constraint holds for both sets.

Figure 21. The 2 maximum paths of the solution.

COMMENT

: Note that the arc constraint will produce the different sequence of consecutives variables that do not contain any 0 . The PATH(MAX) generates all maximum paths of the primal graph. In this case each maximum path corresponds to a connected component of the primal graph.

: This constraint is useful in timetabling problems where the variables are interpreted as the type of job that a person does on consecutive days. Value 0 represents a rest day and one want to impose a cardinality constraint on periods that are located between rest periods. 
: One cannot initially state a cardinality constraint since the rest days are not yet allocated. One can also not use a sliding cardinality constraint since the cardinality constraint does not hold for the sequences of consecutive variables that contains at least one rest day. 


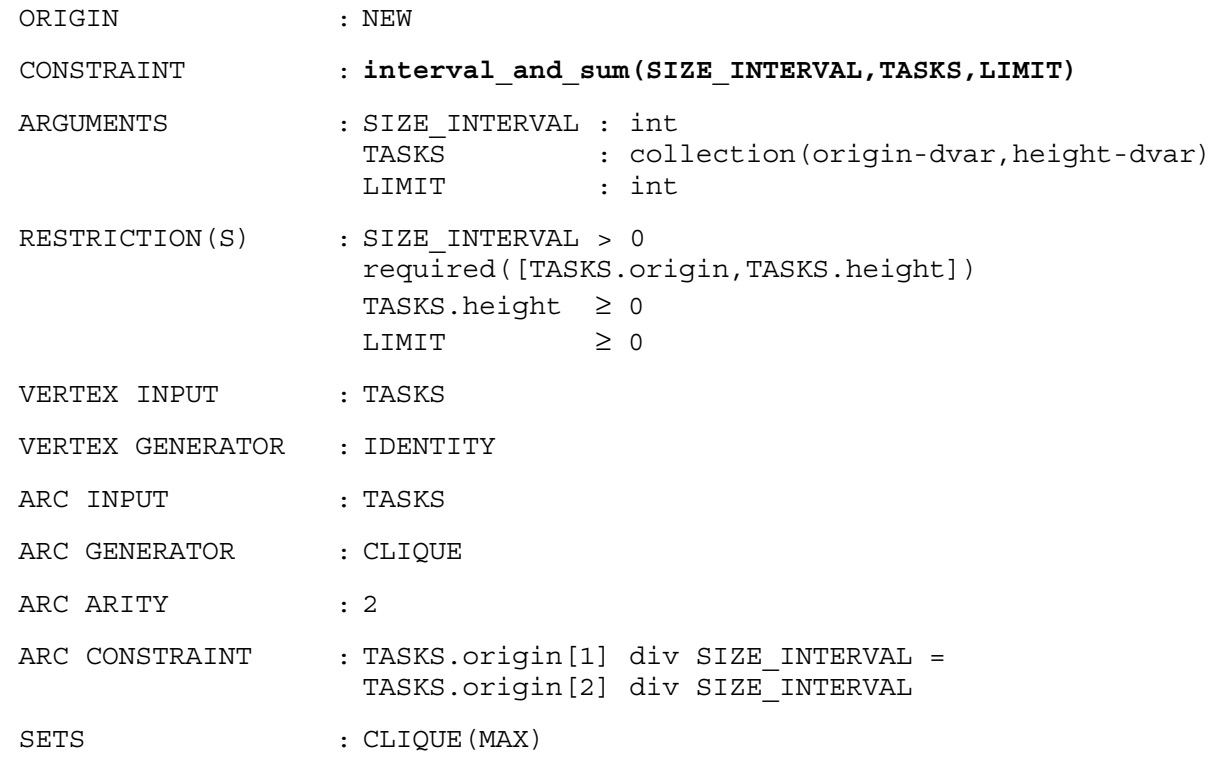

EXAMPLE (S)

: interval_and_sum(5, \{origin-1 height-2, origin-10 height-2, origin-10 height-3, origin-4 height-1\},5)

The left part of the next figure shows the solution associated to the example, while the right part gives the primal graph where the 2 maximum cliques are enclosed by a circle. For each task we indicate its position within the collection. The origin of each task is marked by a dark little square. The 2 cliques correspond respectively to intervals 0.4 and 10..14. The constraint holds since the sum of the heights of the tasks that are located in the same interval do not exceed the limit 5.

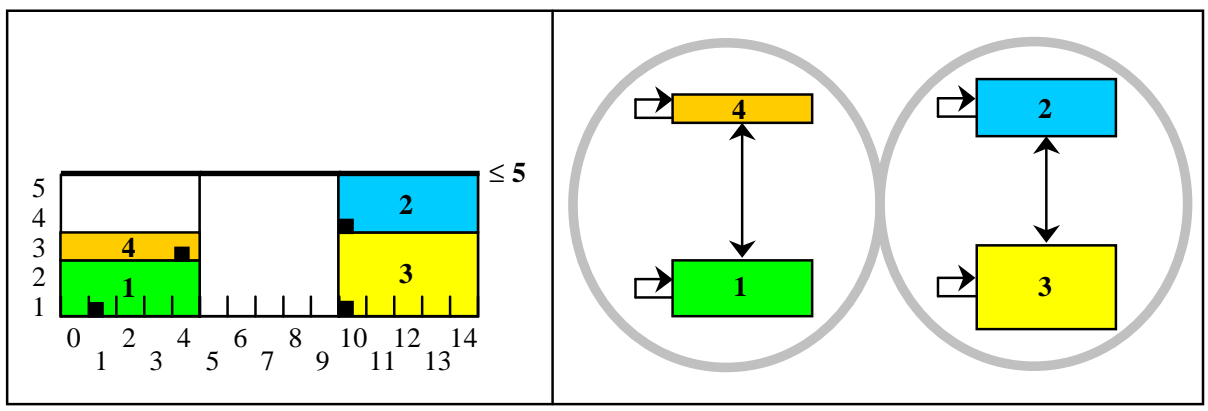

Figure 22. Solution and related cliques associated to intervals $0 . .4$ and 10.14 . case each interval can be interpreted as a morning or an afternoon of different consecutives days. We have a capacity constraint for all tasks that are assigned to the same morning or afternoon of a given day. 
ORIGIN

CONSTRAINT

ARGUMENTS

RESTRICTION (S)

VERTEX INPUT

VERTEX GENERATOR

ARC INPUT

ARC GENERATOR

ARC ARITY

ARC CONSTRAINT

SETS

DYNAMIC CONSTRAINT : Card(TASKS.COlour, COLOURS) $\leq$ ATMOST

PURPOSE

EXAMPLE (S)
: [COUSIN 93]

: interval_and_count (ATMOST,COLOURS,TASKS,SIZE_INTERVAL)

COLOURS : collection (val-int)

TASKS : collection (origin-dvar, colour-dvar)

SIZE_INTERVAL : int

- ATMOST $\geq 0$

required (COLOURS . val)

distinct (COLOURS/val)

required ( [TASKS.origin, TASKS. colour] )

SIZE_INTERVAL > 0

: TASKS

: IDENTITY

: TASKS

: CLIQUE

$: 2$

: TASKS.origin[1] div SIZE_INTERVAL = TASKS.origin[2] div SIZE_INTERVAL

: CLIQUE (MAX) 
: If we would have like to only consider intervals that correspond to the morning or to the afternoon we could have extended the constraint in the following way:

- We introduce 2 extra parameters REST and QUOTIENT that correspond to non-negative integers such that REST is less than QUOTIENT

- We add the following condition to the arc constraint:

(tasks.ORIGIN[1] div SIZE_INTERVAL) $\equiv$ REST mod (QUOTIENT)

Now, if we want to express a constraint on the morning intervals we set REST to 0 and QUOTIENT to 2 . 


\subsection{Proximity constraints}

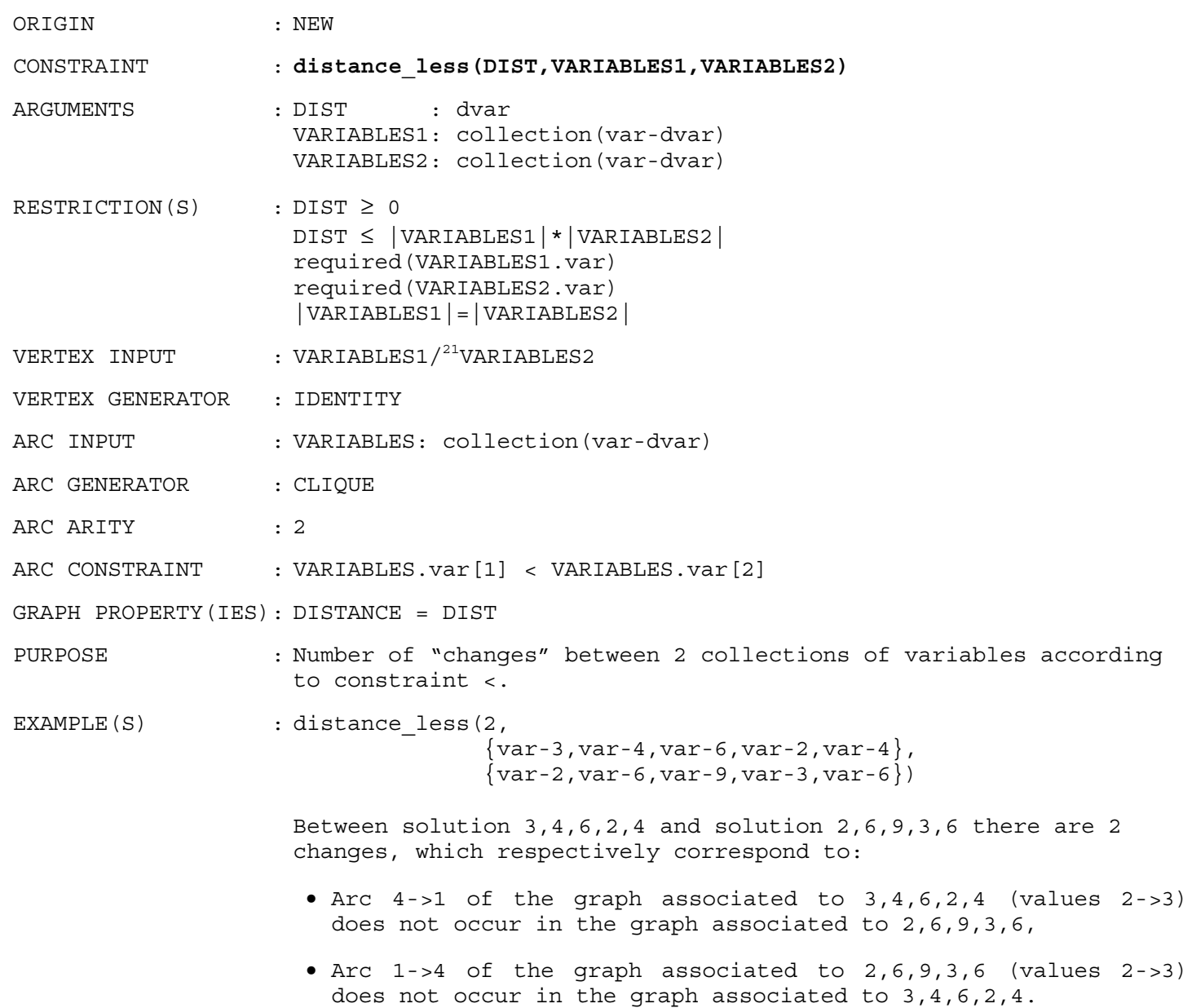

${ }^{21}$ Sign " "” indicates that we generate 2 distinct structured networks. 


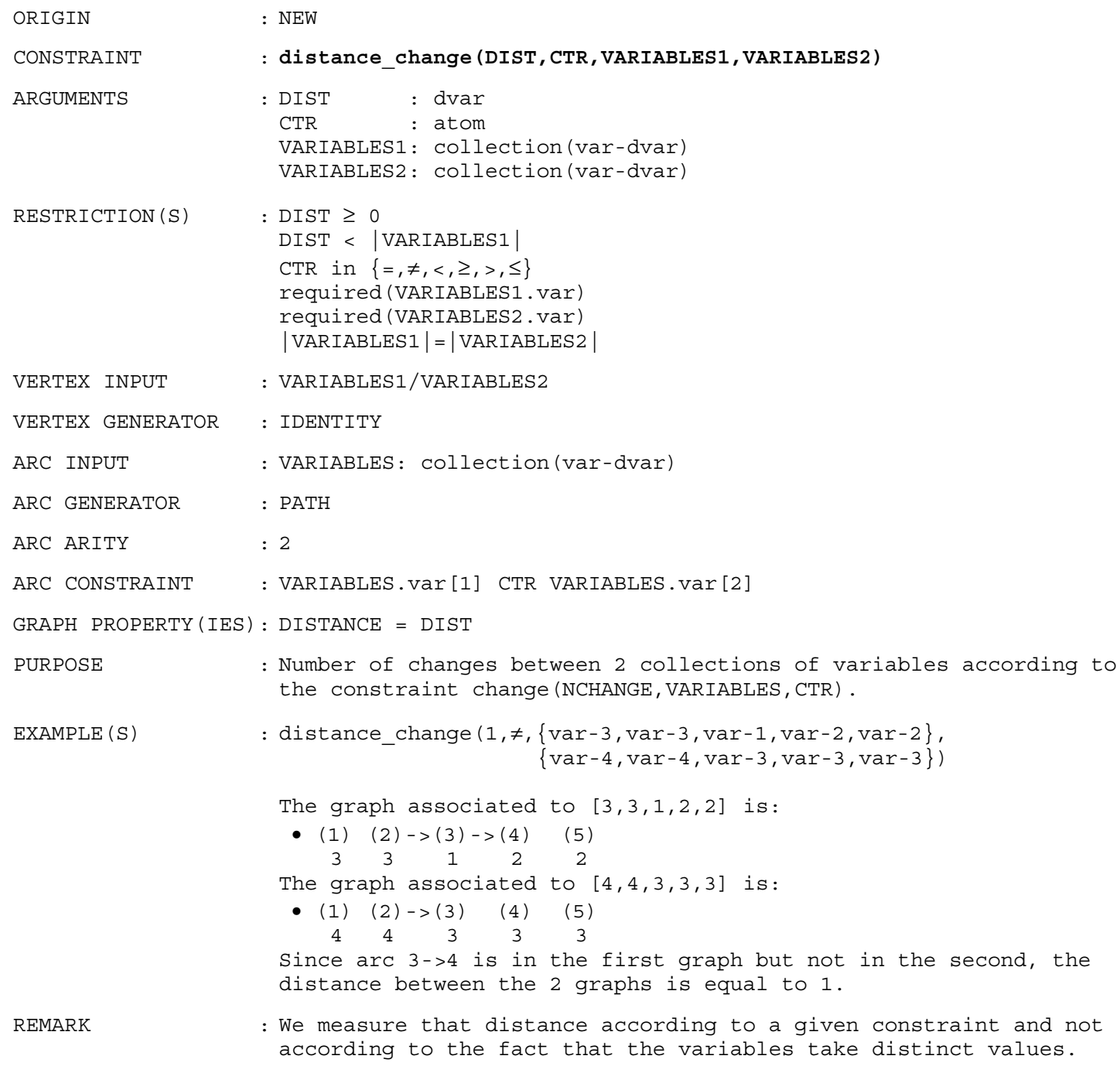

REMARK

: We measure that distance according to a given constraint and not according to the fact that the variables take distinct values. 


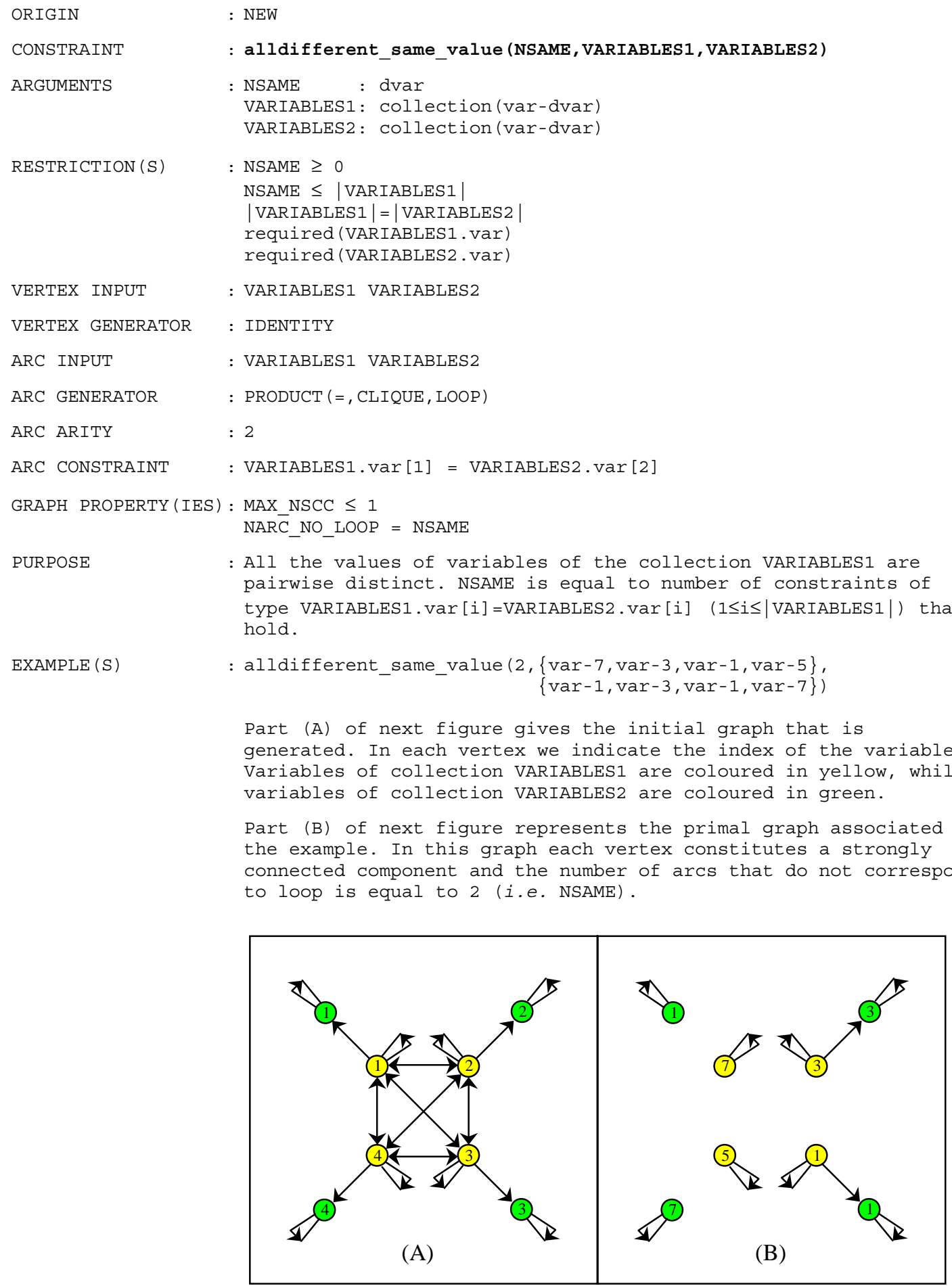

Figure 24. Initial generated graph and primal graph.

: The arc generator PRODUCT(=,CLIQUE, LOOP) is used in order to generate all the arcs in the following way:

- It first generates all links between the items of the first collection VARIABLES1,

- It then generates one loop for all items of the second collection VARIABLES2,

- Finally it generates the arc associated to the product. Since we use the restriction $=$, it only creates an arc between the items of same rank. 
: When all variables of the second collection are initially bound and pairwise different, then this constraint can be interpreted in the following way:

- We consider the variables of the second collection to be a previous solution of a problem where all variables have to be distinct.

- We consider the variables of the first collection to be a current solution to find, where all variables should be pairwise distinct.

The variable NSAME mesures the "distance" of the current solution from the previous solution. 


\subsection{Graph constraints}

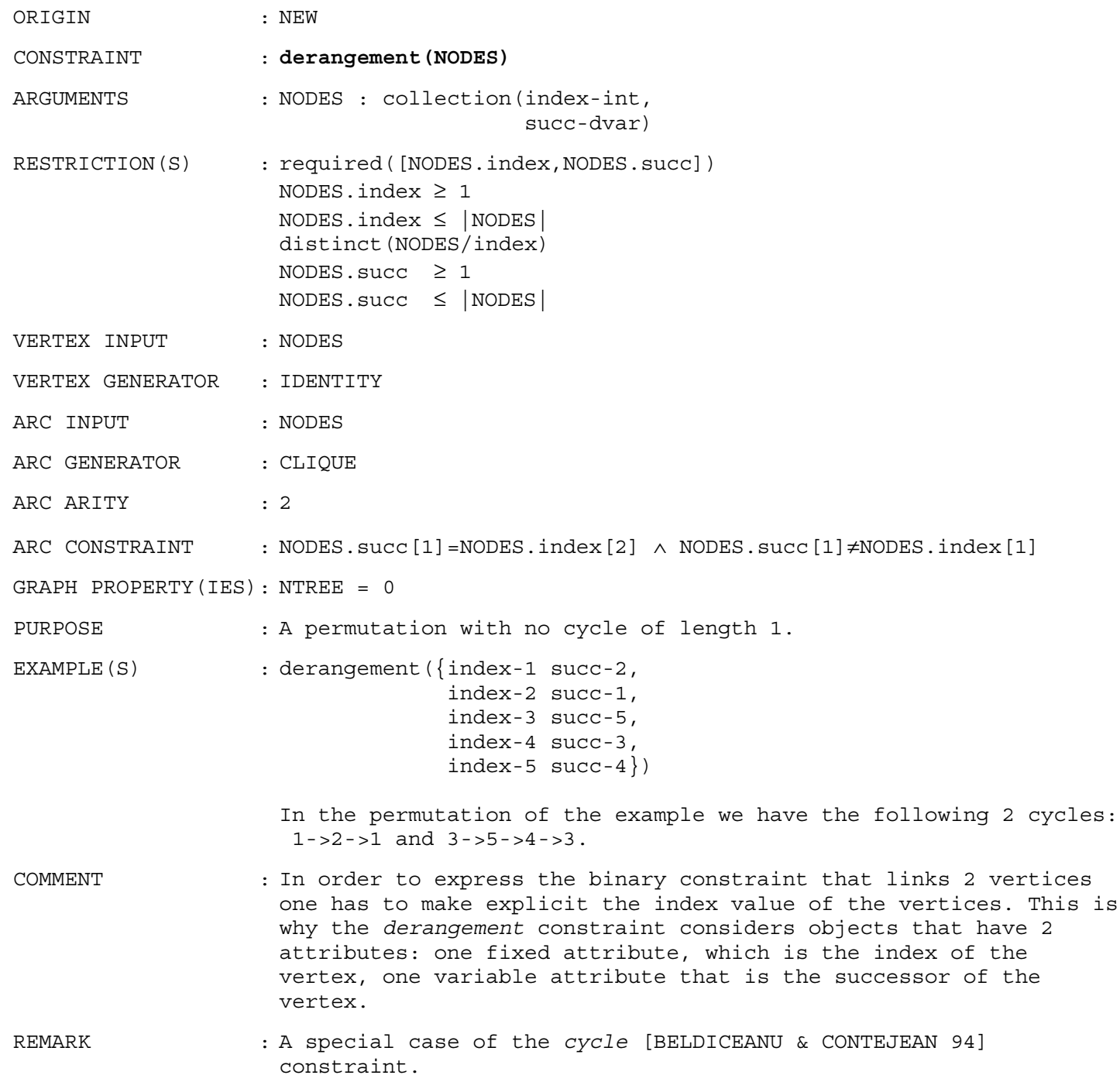

COMMENT

: In order to express the binary constraint that links 2 vertices one has to make explicit the index value of the vertices. This is why the derangement constraint considers objects that have 2 attributes: one fixed attribute, which is the index of the vertex, one variable attribute that is the successor of the vertex.

REMARK

: A special case of the cycle [BELDICEANU \& CONTEJEAN 94] constraint. 


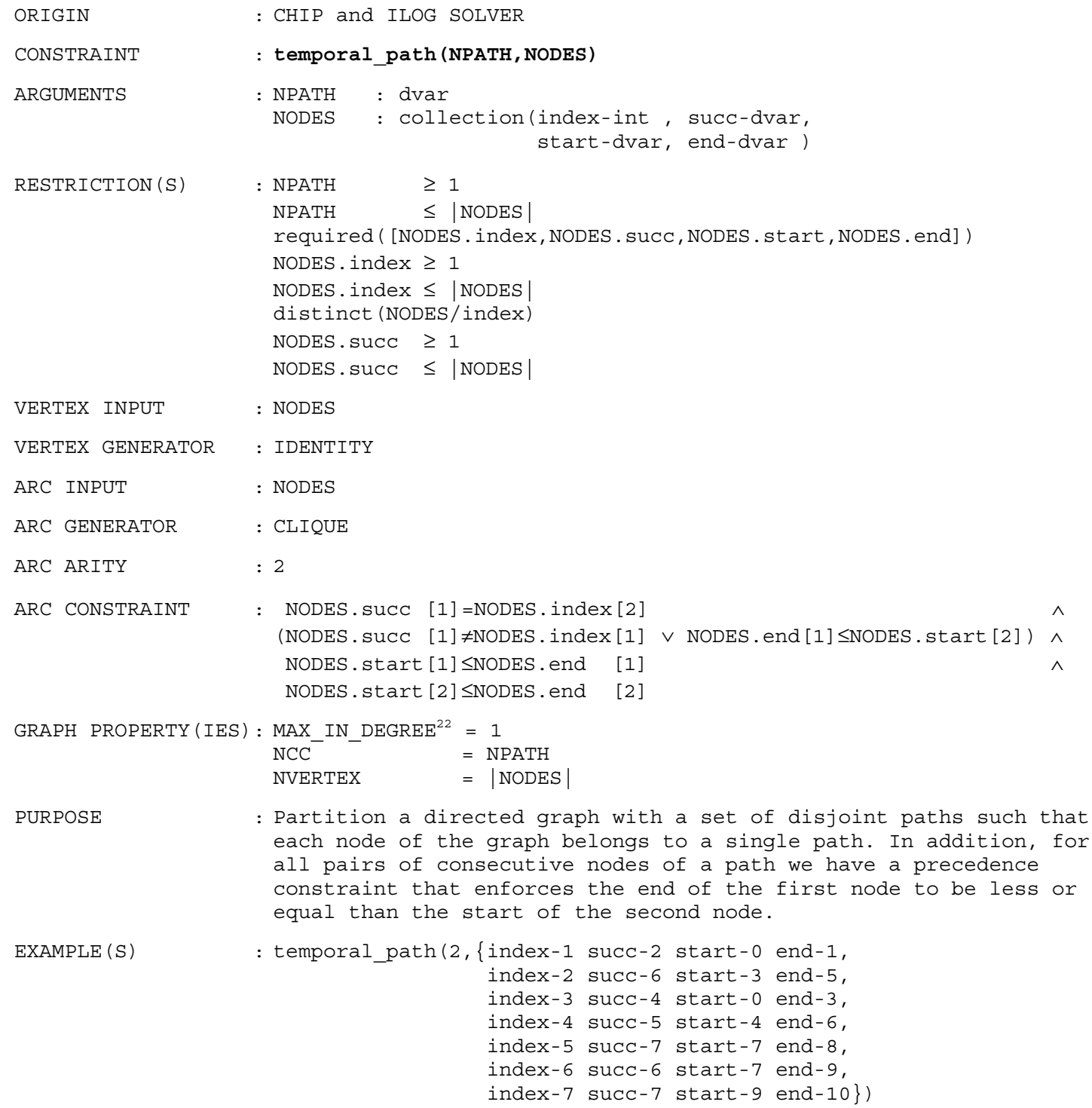

PURPOSE

: Partition a directed graph with a set of disjoint paths such that each node of the graph belongs to a single path. In addition, for all pairs of consecutive nodes of a path we have a precedence constraint that enforces the end of the first node to be less or equal than the start of the second node.

$\operatorname{EXAMPLE}(S)$

: temporal_path $(2,\{$ index-1 succ-2 start-0 end-1, index-2 succ-6 start-3 end-5

index-3 succ-4 start-0 end-3, index-4 succ-5 start-4 end- 6 , index-5 succ-7 start-7 end-8,

The next figure shows the 2 paths associated to the example.

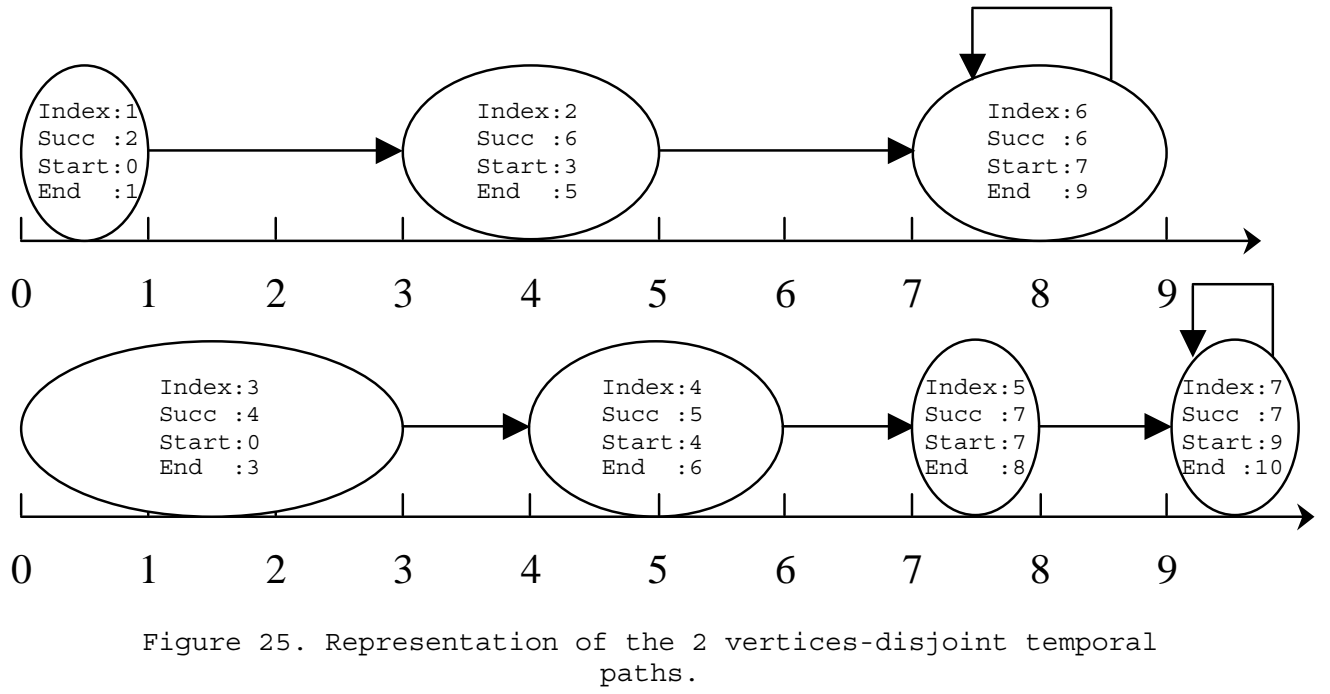

\footnotetext{
${ }^{22}$ MAX_IN_DEGREE was defined as the maximum number of predecessors without considering an arc from a vertex to itself.
} 
: The arc constraint is a conjunction of 4 conditions that respectively corresponds to:

- A constraint that links the successor variable to the index,

- A precedence constraint that applies on one node and its successor

- One precedence constraint between the start and the end of the node that corresponds to the departure of an arc,

- One precedence constraint between the start and the end of the node that corresponds to the arrival of an arc.

We use 3 graph properties in order to enforce the partitioning of the graph in distinct paths.

- The first property "MAX IN DEGREE=1" enforces that each node has only one single predecessor (except the last node of a path which has also itself as a predecessor),

- The second property "NCC=NPATH" ensures that we have the required number of paths,

- The third property "NVERTEX=|NODES|" makes sure that for each node, the start is not after the end.

REMARK

: This constraint is related to the path constraint of Ilog solver. It can also be directly expressed with the cycle [BELDICEANU \& CONTEJEAN 94] constraint of CHIP by using the diff nodes and the origin parameters.

A generic model that handles paths, trees and cycles is presented in [LABBÉ, LAPORTE \& RODRÍGUEZ-MARTÍN 98]. 


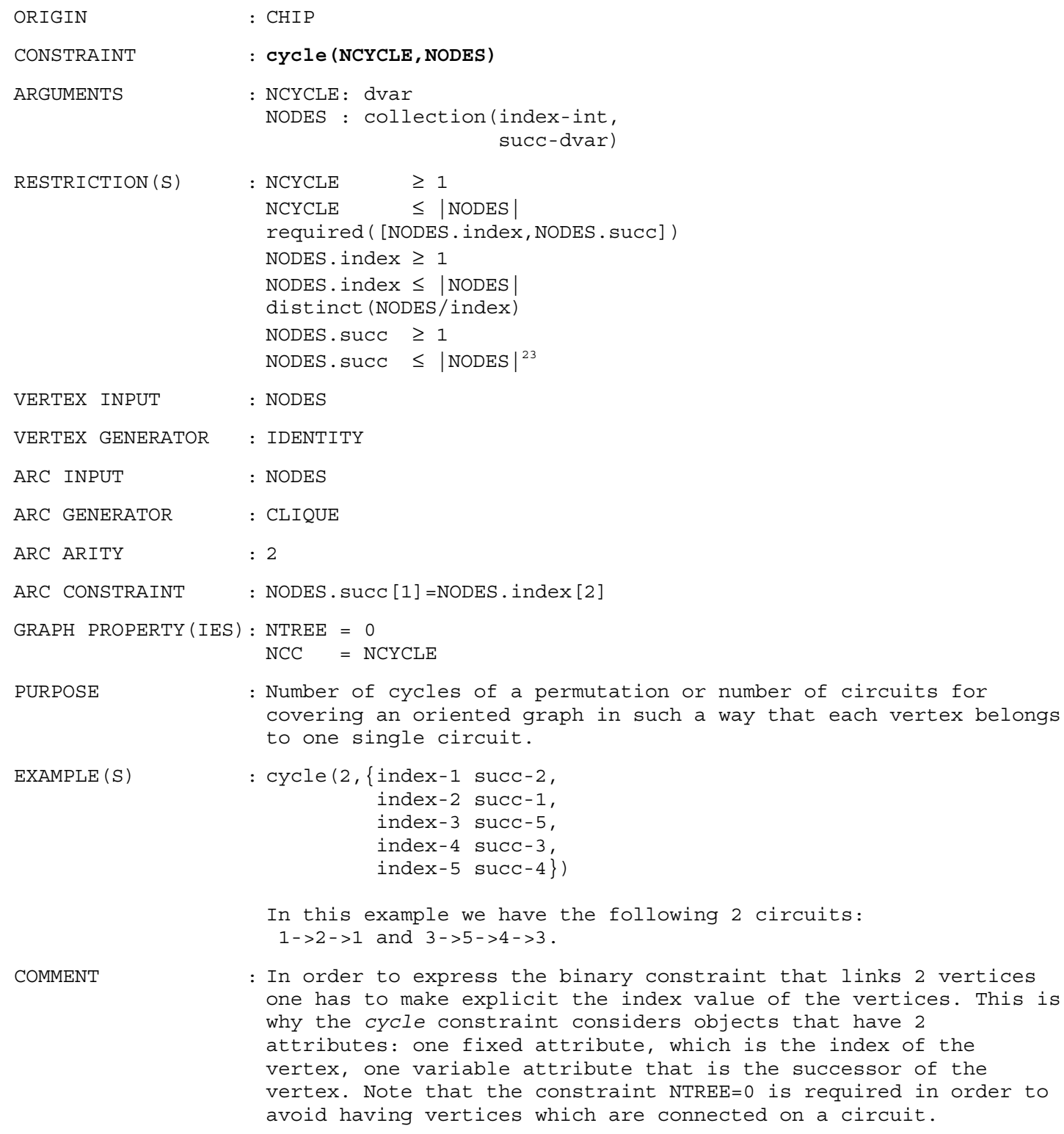

COMMENT : In order to express the binary constraint that links 2 vertices one has to make explicit the index value of the vertices. This is why the cycle constraint considers objects that have 2 attributes: one fixed attribute, which is the index of the vertex, one variable attribute that is the successor of the vertex. Note that the constraint $\mathrm{NTREE}=0$ is required in order to avoid having vertices which are connected on a circuit.

\footnotetext{
${ }^{23}$ From the previous restrictions and from the arc constraint one can deduce that there will be a bijection from the successor variables to the values 1 to the total number of vertices. If these restrictions were not explicitly provided then it would be impossible to derive this property.
} 


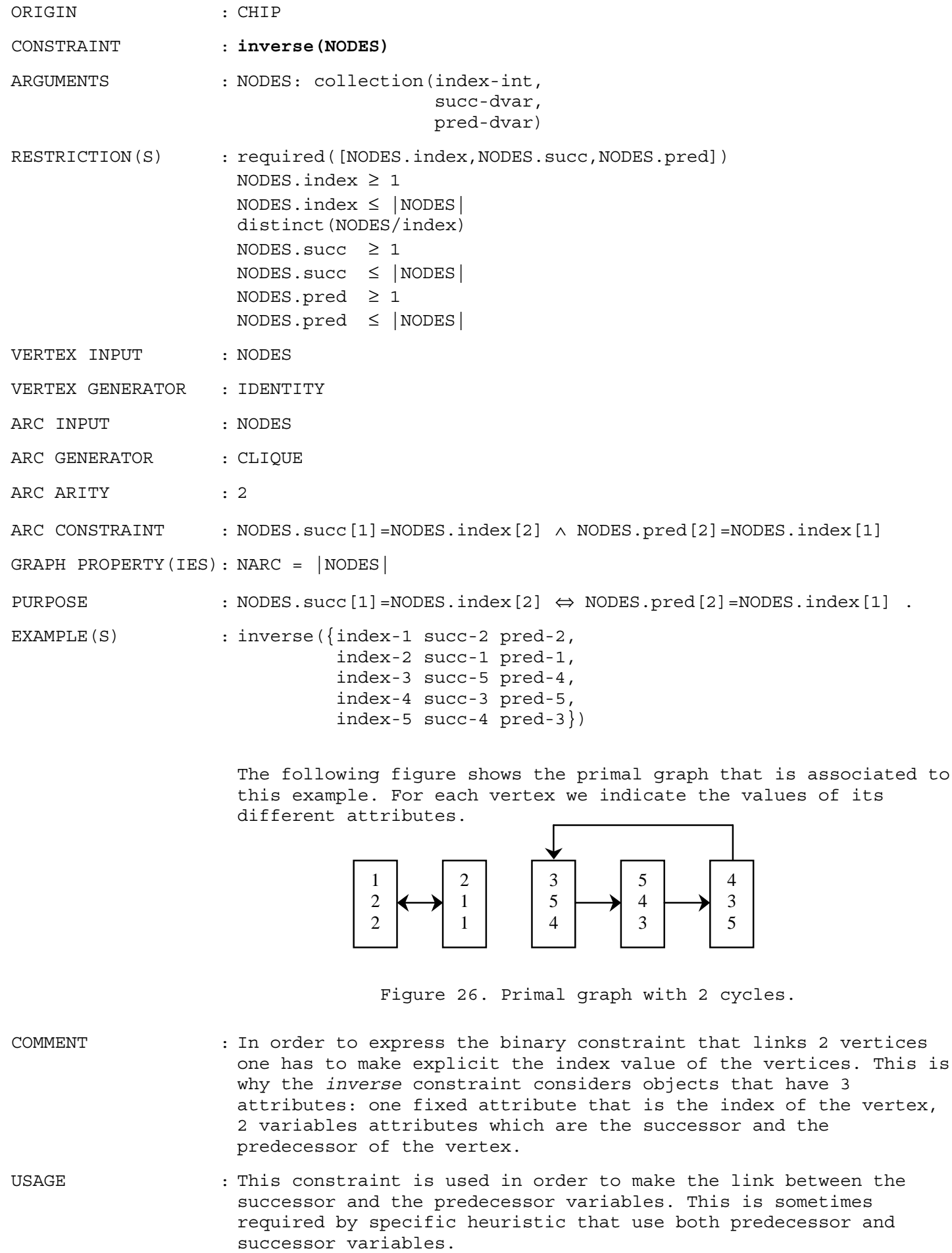

The following figure shows the primal graph that is associated to this example. For each vertex we indicate the values of its different attributes.

Figure 26. Primal graph with 2 cycles.

COMMENT

USAGE

: In order to express the binary constraint that links 2 vertices one has to make explicit the index value of the vertices. This is why the inverse constraint considers objects that have 3 attributes: one fixed attribute that is the index of the vertex, 2 variables attributes which are the successor and the predecessor of the vertex.

: This constraint is used in order to make the link between the successor and the predecessor variables. This is sometimes required by specific heuristic that use both predecessor and successor variables. 
ORIGIN

CONSTRAINT

ARGUMENTS

RESTRICTION (S)

EXAMPLE (S)

VERTEX INPUT

ARC INPUT

ARC GENERATOR

ARC ARITY

PURPOSE
: [RÉGIN 99]

\section{symmetric_alldiff (NODES)}

: NODES: collection(index-int, succ-dvar)

: required ([NODES.index, NODES.sucC])

NODES. index $\geq 1$

NODES. index $\leq \mid$ NODES $\mid$

distinct (NODES/index)

NODES.SucC $\geq 1$

NODES.sucC $\leq \mid$ NODES $\mid$

: NODES

VERTEX GENERATOR

: IDENTITY

: NODES

: CLIQUE $(\neq)$

: 2

ARC CONSTRAINT

: NODES. succ [1]=NODES. index[2] $\wedge$ NODES. succ [2] =NODES . index [1]
NODES

: An alldifferent constraint plus the fact that you have to group variables by pairs: if variable i takes value $j$ then variable $j$ takes value $i$. It can also be interpreted as a graph-covering problem where one has to cover a directed graph with circuits of length 2 .

: symmetric_alldiff( $\{$ index-1 $\operatorname{succ}-3$,

index-2 succ-4,

index-3 succ-1,

index-4 succ-2\})

The following figure shows the primal graph that is associated to this example. For each vertex we display its index and its corresponding value below.
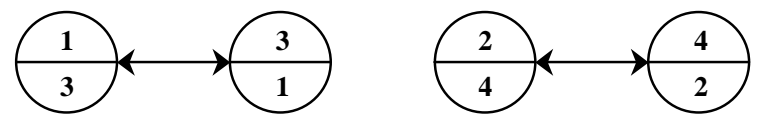

Figure 27. Primal graph with 2 pairs of vertices.

COMMENT

: In order to express the binary constraint that links 2 vertices one has to make explicit the index value of the vertices.

USAGE

: As it was reported in [RÉGIN 99, page 420], this constraint is useful to express matches between persons.

This constraint also appears implicitly in the cycle cover problem and corresponds to the 4 conditions given in section 1 "Modeling the Cycle Cover Problem" of [PESANT \& SORIANO 98].

REMARK

: From a modeling point of view this constraint can be express with the cycle constraint [BELDICEANU \& CONTEJEAN 94, page 115] where one imposes the additional condition that each cycle has only 2 nodes. 


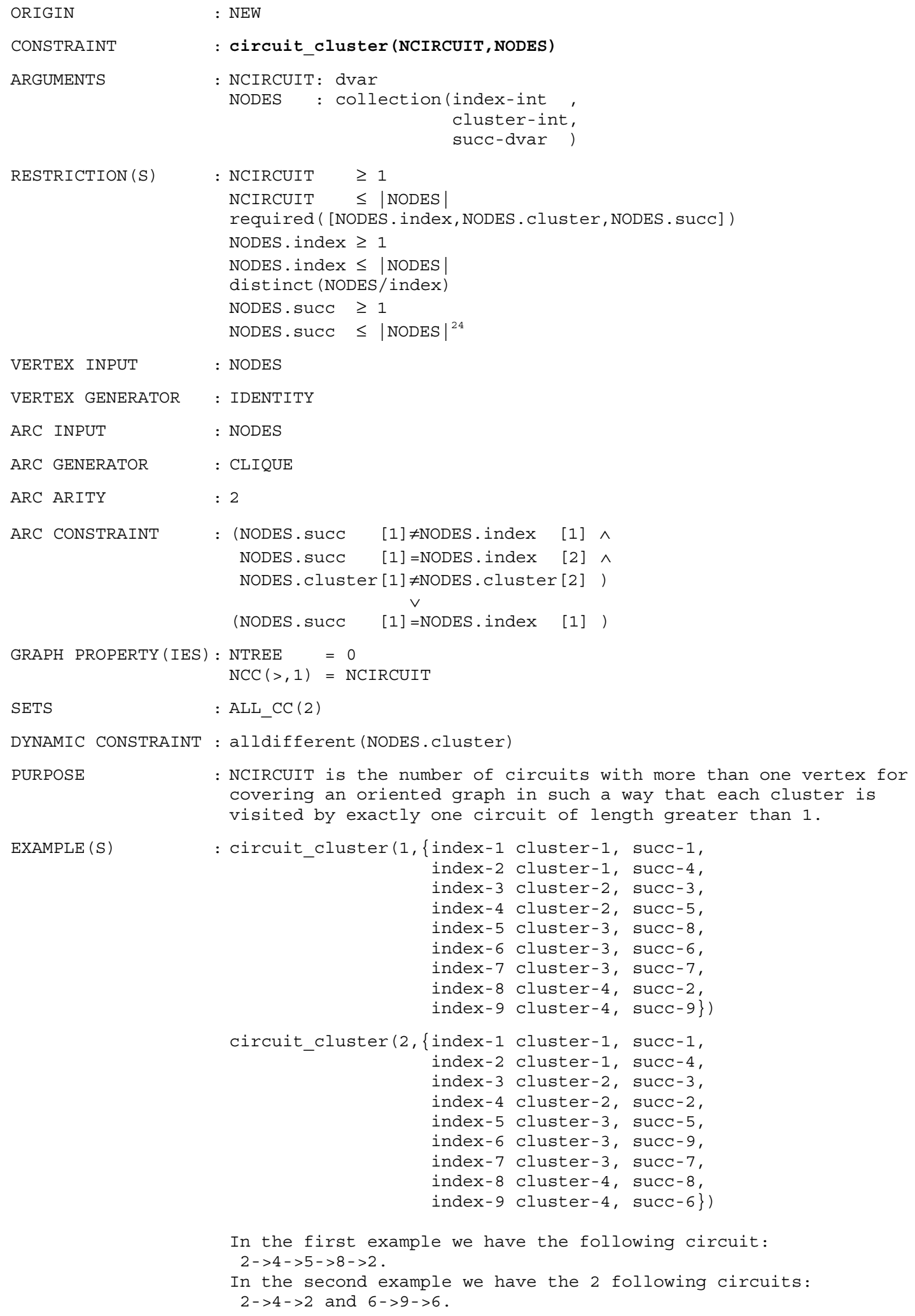

$\operatorname{EXAMPLE}(\mathrm{S})$

\footnotetext{
${ }^{24}$ From the previous restrictions and from the arc constraint one can deduce that there will be a bijection from the successor variables to the values 1 to the total number of vertices. If these restrictions were not explicitly provided then it would be impossible to derive this property.
} 
The parts (A) and (B) of next figure represent the nodes and the circuits associated to the 2 examples. For each node we give its different attributes (i.e. the index of the node, the cluster to which the node belongs and the successor of the node). Each cluster is surrounded by a rectangle. Each dynamic set is represented in grey.

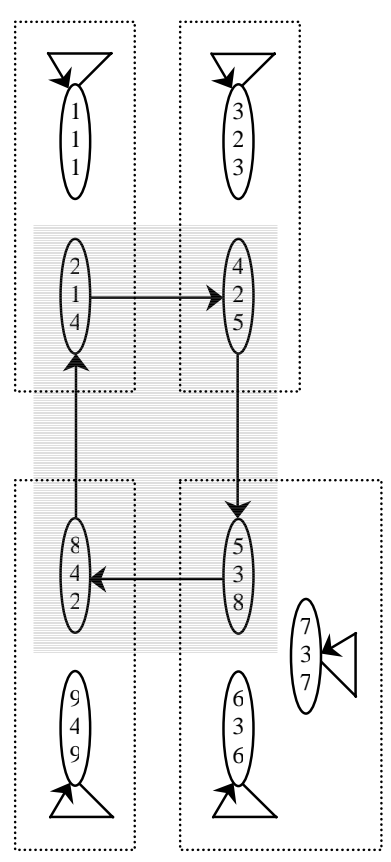

(A)

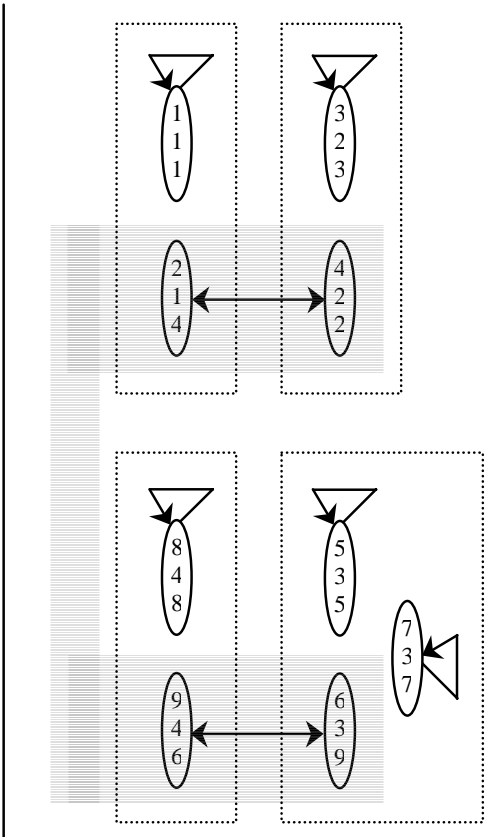

(B)

Figure 28. Covering clusters with circuits.

COMMENT

USAGE

: In order to express the binary constraint that links 2 vertices one has to make explicit the index value and the cluster of the vertices. This is why the cycle constraint considers objects that have 3 attributes: 2 fixed attributes, which are the index of the vertex and the cluster to which the vertex belongs, one variable attribute that is the successor of the vertex. The partitioning of the clusters by different circuits is expressed by:

- The graph property "NTREE=0" is required in order to avoid having vertices which are connected on a circuit.

- The graph property "NCC $(>, 1)=$ NCIRCUIT" express the fact that the number of connected components, such that the number of nodes is greater than 1 , is equal to the domain variable NCIRCUIT.

- The dynamic constraint "alldifferent (NODES.cluster)" on the set "ALL_CC(2)" states for all connected components of at least 2 nodes, that the cluster attributes should be pairwise distinct.

: A related abstraction in operation research was introduced in [LAPORTE, ASEF-VAZIRI \& SRISKANDARAJAH 96]. It was reported as the Generalized Travelling Salesman Problem (GTSP). The circuit cluster constraint differs from the GTSP because of the 2 following points:

- Each node of our graph belongs to one single cluster,

- We do not constrain the number of circuits to be equal to 1: the number of circuits should be equal to one of the values of the domain of the variable NCIRCUIT. 


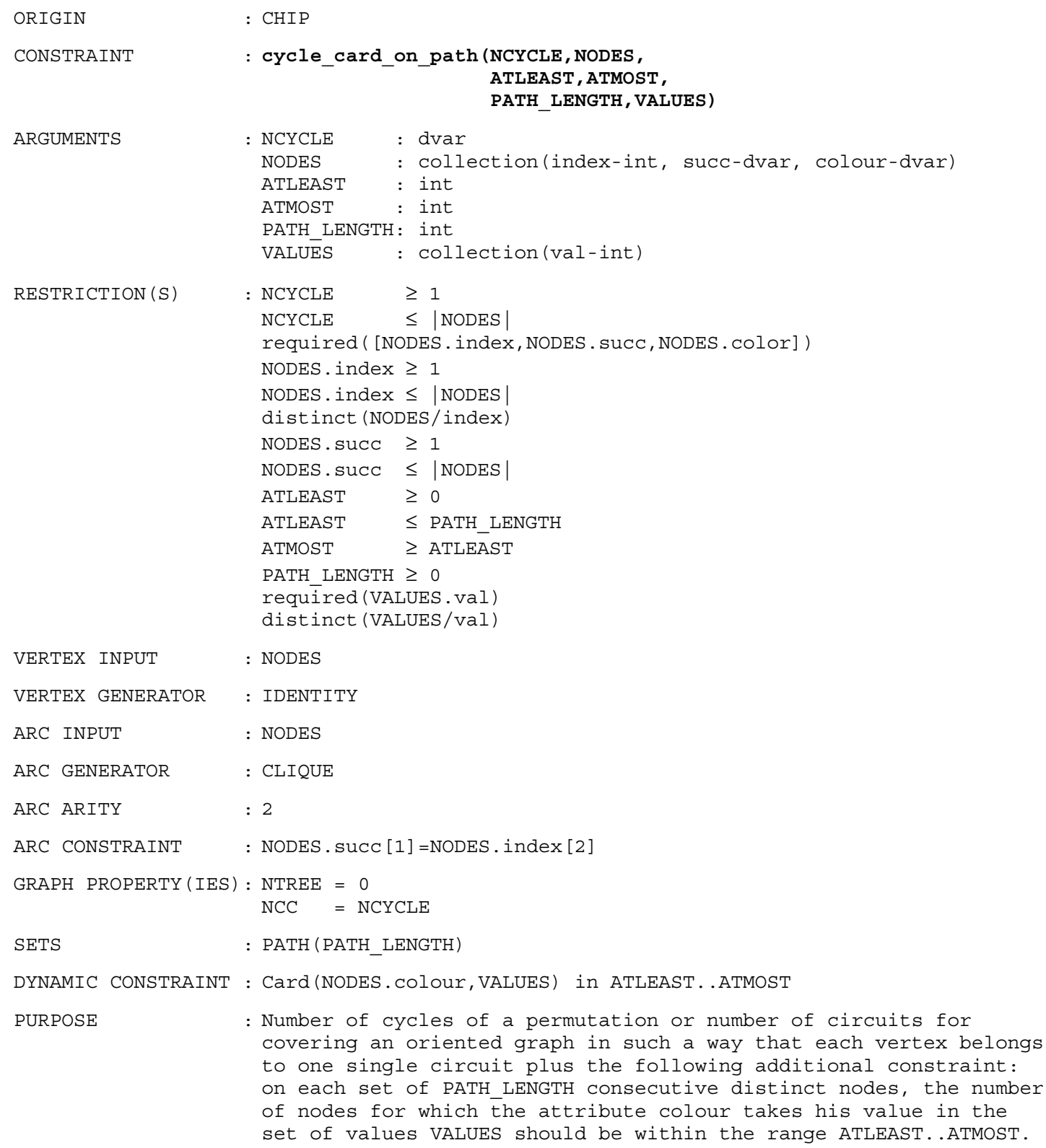


\{index-1 succ-7 colour-2,

index-2 succ-4 colour-3,

index-3 succ-8 colour-2,

index-4 succ-9 colour-1,

index-5 succ-1 colour-2,

index-6 succ-2 colour-1,

index-7 succ-5 colour-1,

index-8 succ-6 colour-1,

index-9 succ-3 colour-1\},

1 ,

2 ,

3,

$\{\operatorname{val}-1\}$

The next figure shows the 2 cycles associated to the previous example. An ellipse stresses each set of 3 consecutive nodes. In each set, the blue colour (i.e. the colour associated to value 1 ) occurs at least once and at most twice.
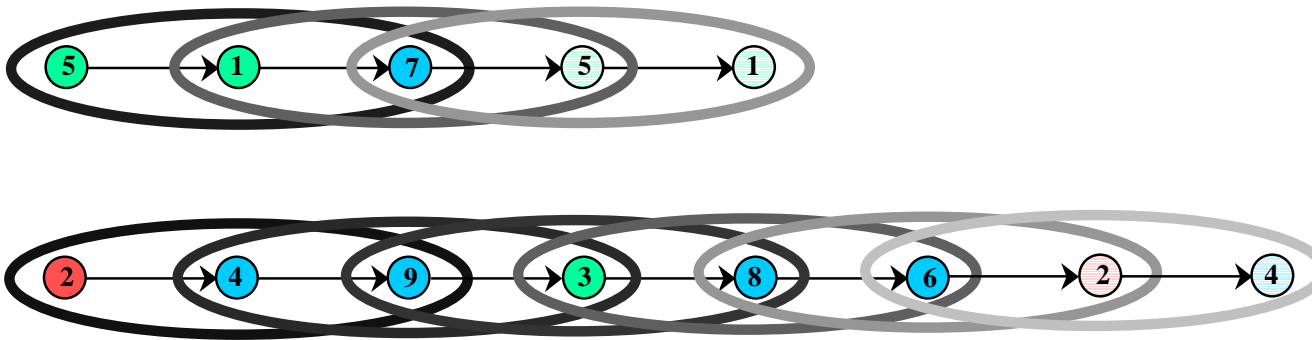

Figure 29. The 2 vertices-disjoint cycles and of the corresponding different paths of length 3 .

: This constraint is a special case of the sequence parameter of the cycle constraint [BOURREAU 99, pages 121-128] of CHIP. 


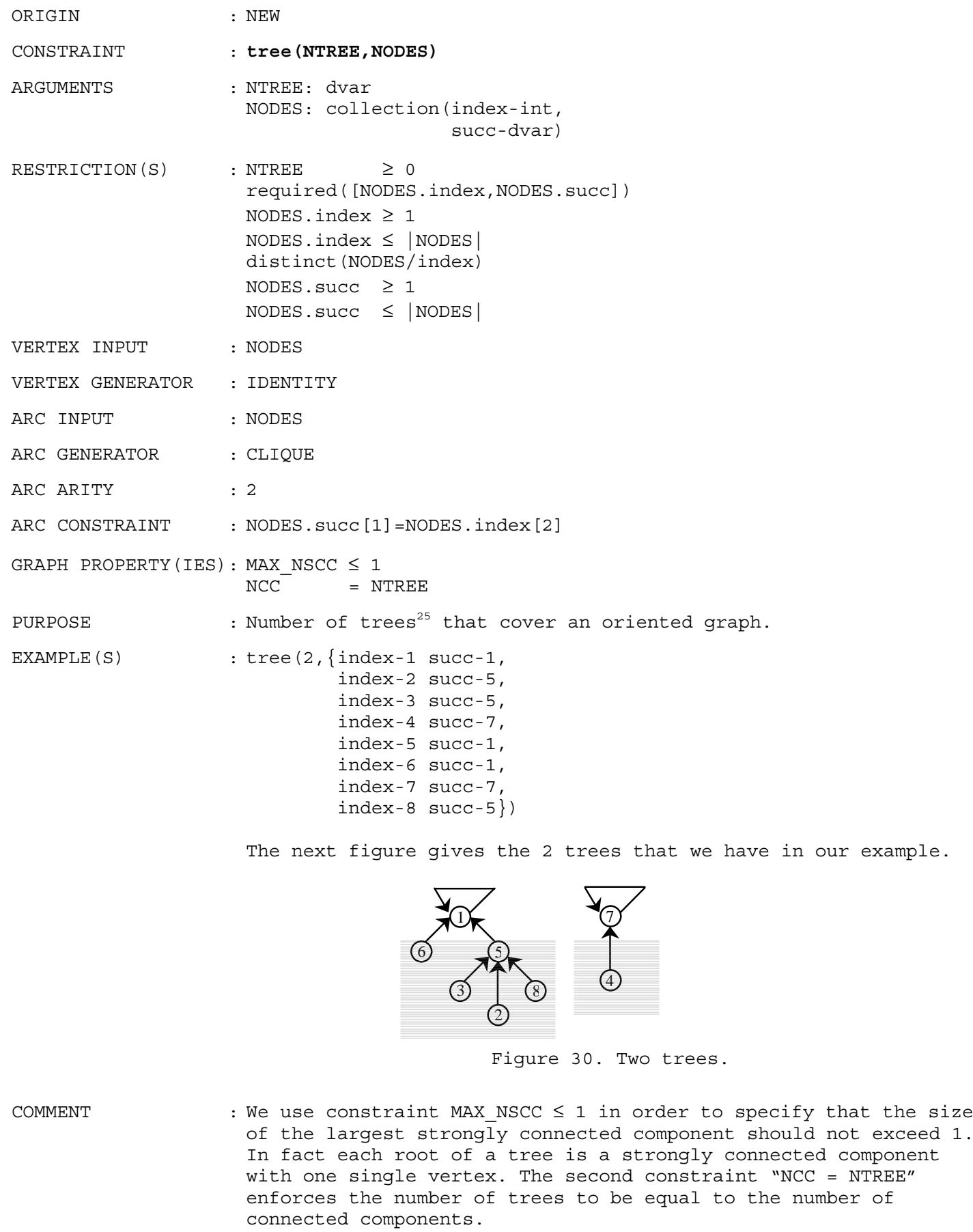

The next figure gives the 2 trees that we have in our example.

Figure 30. Two trees.

COMMENT
We use constraint MAX NSCC $\leq 1$ in order to specify that the size of the largest strongly connected component should not exceed 1 . In fact each root of a tree is a strongly connected component with one single vertex. The second constraint "NCC = NTREE" enforces the number of trees to be equal to the number of connected components.

\footnotetext{
${ }^{25}$ Strictly speaking we should say anti-arborescence instead of tree since the edges are directed from the leaves to the root.
} 


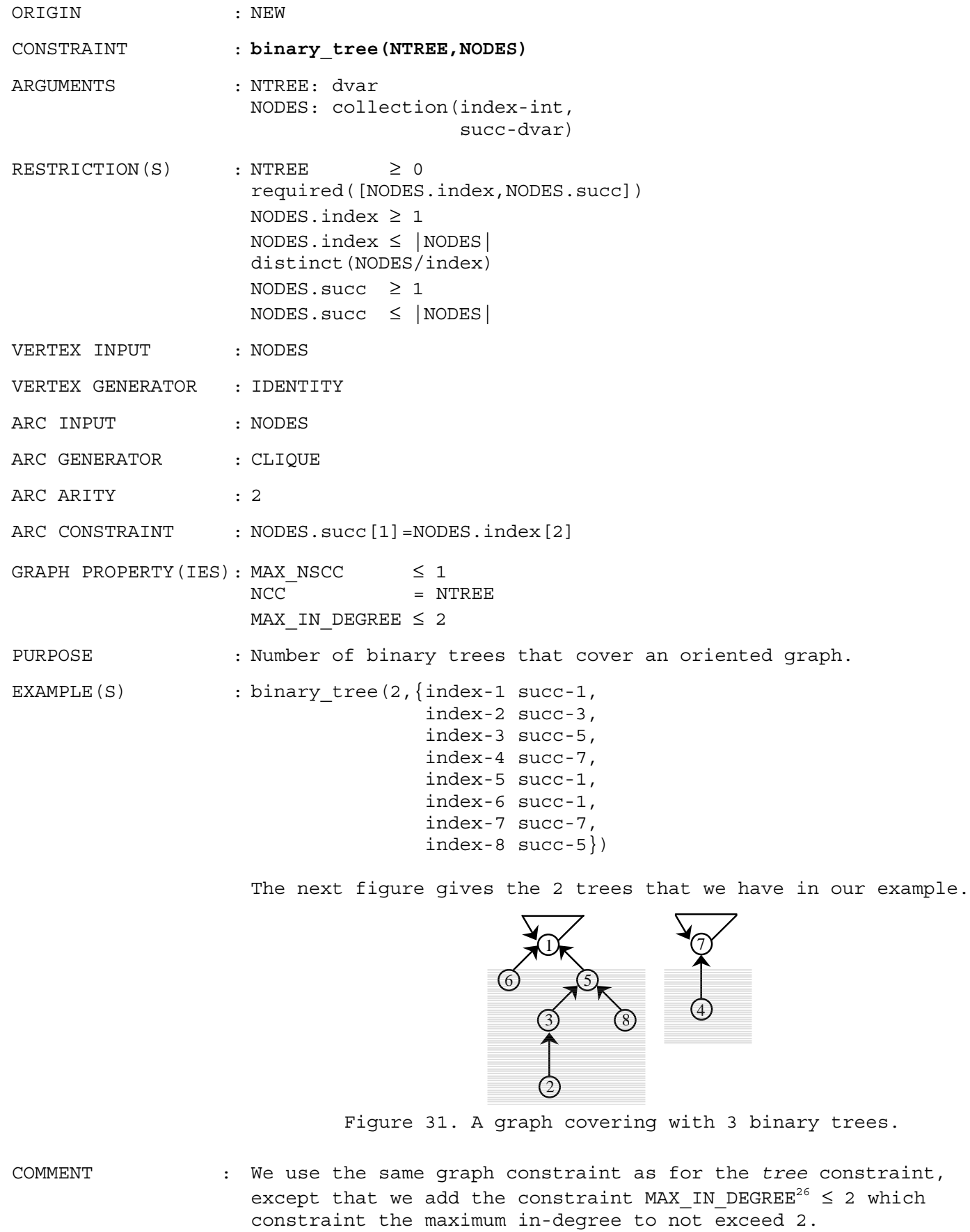

The next figure gives the 2 trees that we have in our example.

Figure 31. A graph covering with 3 binary trees.

COMMENT

We use the same graph constraint as for the tree constraint, except that we add the constraint MAX_IN_DEGREE ${ }^{26} \leq 2$ which constraint the maximum in-degree to not exceed 2.

${ }^{26}$ MAX_IN_DEGREE do not consider loops; this is why we do not have problem with the root of the trees. 


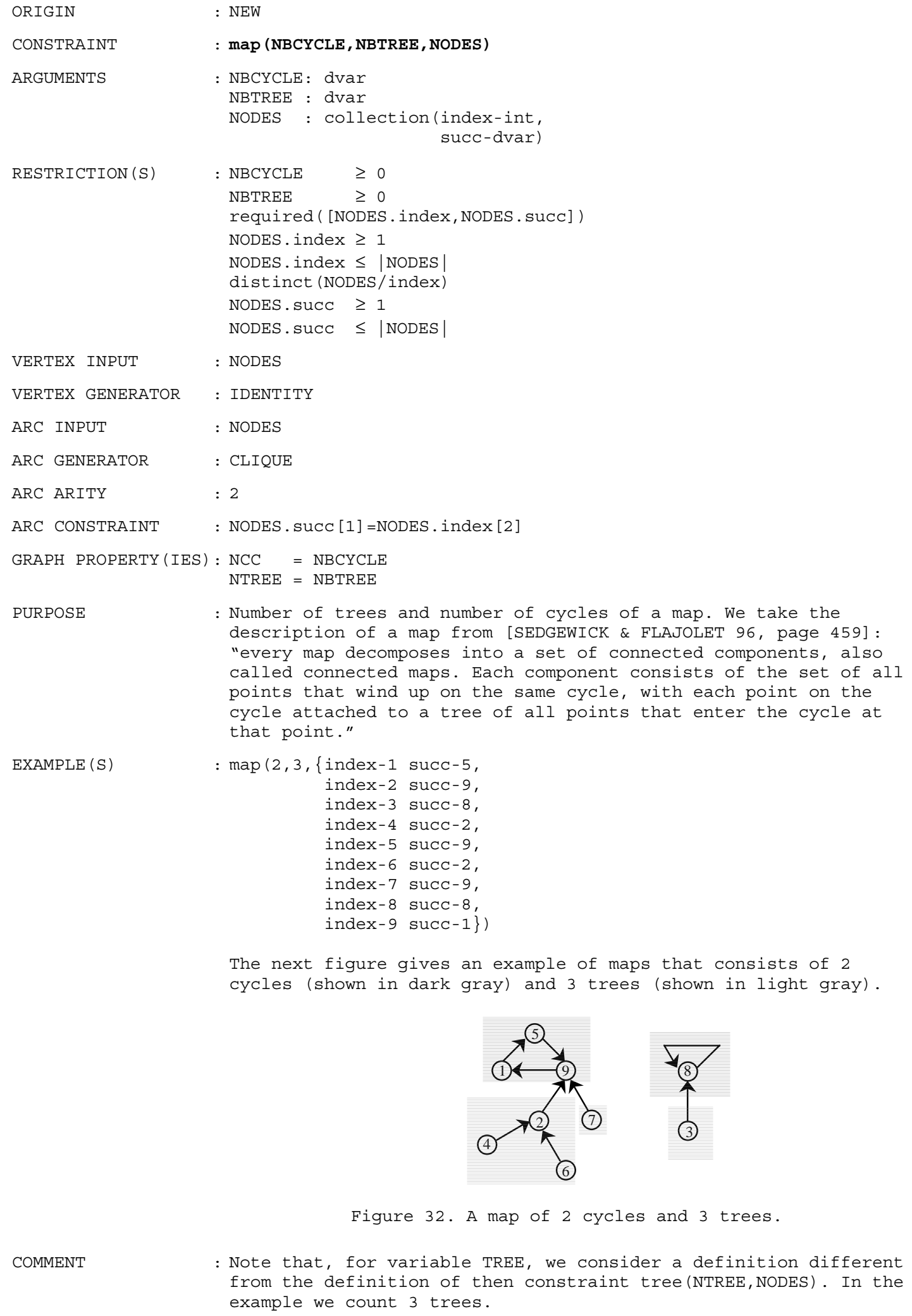

The next figure gives an example of maps that consists of 2 cycles (shown in dark gray) and 3 trees (shown in light gray).

Figure 32. A map of 2 cycles and 3 trees. from the definition of then constraint tree (NTREE, NODES). In the example we count 3 trees. 


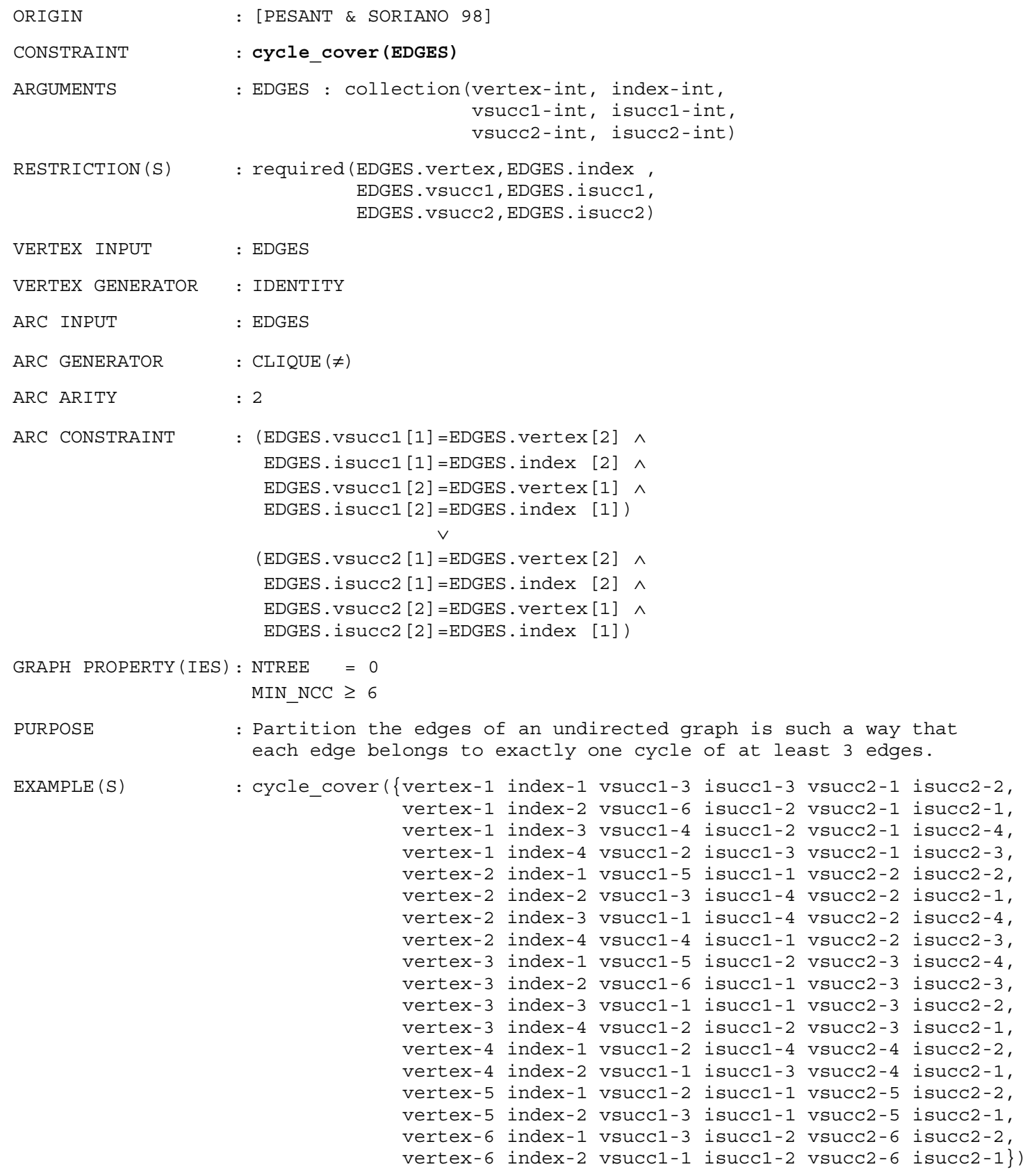


The part (A) of next figure gives the initial graph for which we want to find out a cycle covering. All the edges incident to a given vertex are clockwise labelled from 1 to the degree of that vertex. The part (B) shows a cycle cover of the graph of part $A$.

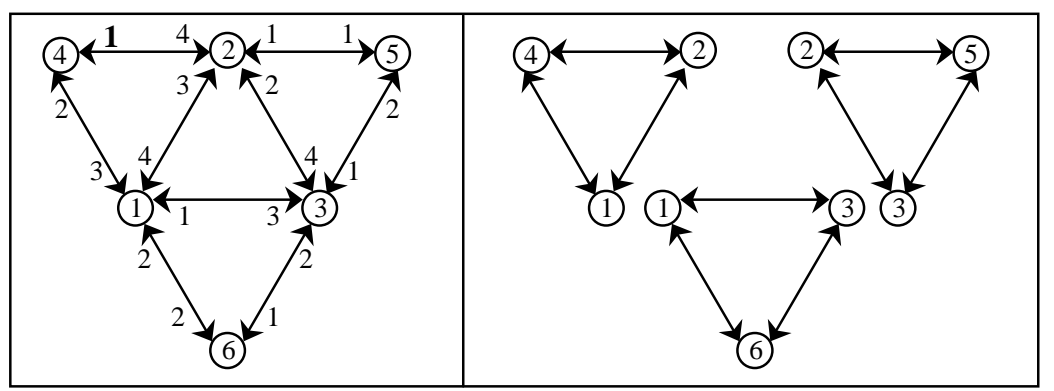

(A)

(B)

Figure 33. A graph and its cycle cover.

For each edge endpoints we create an object with the following attributes:

- VERTEX: the index of the vertex corresponding to the endpoint,

- INDEX : the label associated to the edge endpoints,

- VSUCC1: the index of the vertex corresponding to the opposite endpoint,

- ISUCC1: the label associated to the opposite edge endpoints,

- VSUCC2: the index of the vertex where the covering will go next,

- ISUCC2: the label associated to the edge endpoints where the covering will go next.

For instance, the edge endpoint, which is in bold in the previous figure, has the following attributes:

- VERTEX:4, INDEX:1, VSUCC1:2, ISUCC1:4, VSUCC2:4, ISUCC2:2.

The next figure gives the primal graph associated to the solution shown in part (B). Each node of the primal graph is the endpoint of an edge of the initial graph of part (A). In each node we indicate the first 2 attributes of the corresponding edge: the vertex and the index attributes.
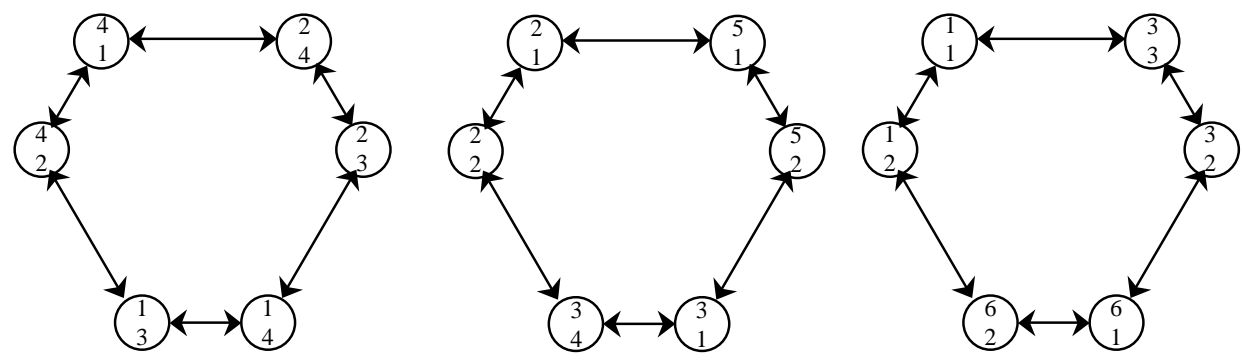

Figure 34. Primal graph associated to the cycle_cover constraint.

: The cycle_cover constraint corresponds to the constraint that was described in [PESANT \& SORIANO 98] for handling the cycle cover problem. We use the same model of the cycle cover problem: to each edge correspond 2 "edge-nodes". However here, we make explicit all attributes of an edge node.

: The motivation given for this constraint in [PESANT \& SORIANO 98] was for telecommunications network design. 


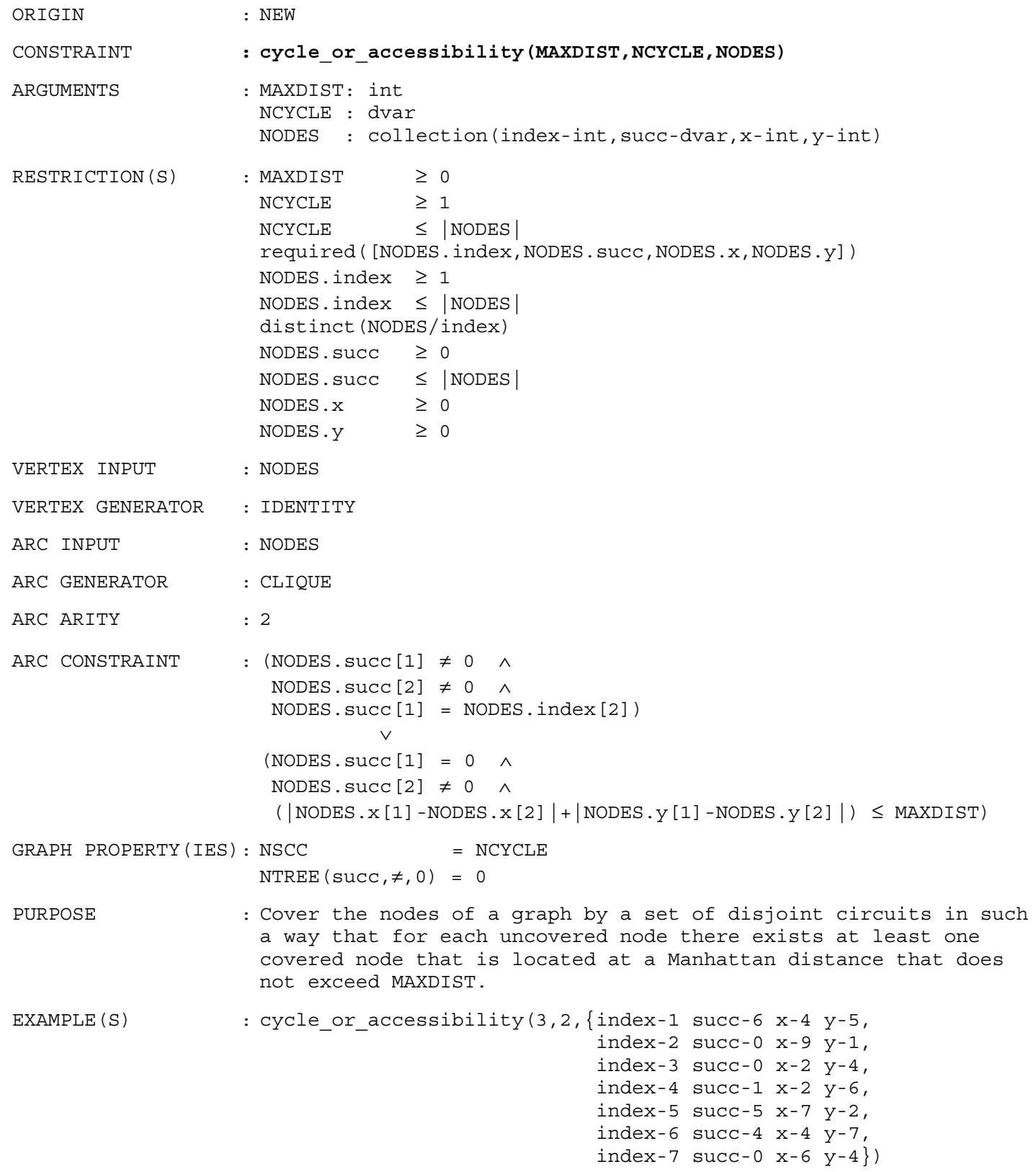

EXAMPLE (S)

: cycle or accessibility $(3,2,\{$ index-1 succ- $6 x-4$ y-5, index-2 succ-0 $x-9 y-1$, index-3 succ-0 $x-2$ y-4, index-4 succ-1 $x-2 \quad y-6$, index-5 succ-5 $x-7 \quad y-2$, index-6 succ- $4 x-4 \quad y-7$ index-7 succ-0 $x-6 \quad y-4\}$ )

The next figure represents the solution associated to the previous example. The covered nodes are colored in gray while the links starting from the uncovered nodes are dashed. In the solution we have 2 circuits and 3 uncovered nodes. All the uncovered nodes are located at a distance that does not exceed 3 from at least one covered node.

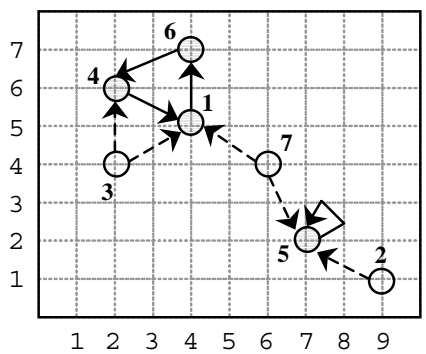

Figure 35. Primal graph associated to the facilities location problem. 
- INDEX: the label associated to the node,

- SUCC : if the node is not directly covered by a circuit then 0 ; if the node is directly covered by a circuit then index of the successor of the node.

- $\mathrm{X}$ : the $\mathrm{x}$-coordinate of the node,

- Y : the y-coordinate of the node.

REMARK

: This kind of facilities location problem is described in [LABBÉ, LAPORTE \& RODRÍGUEZ-MARTÍN 98, pages 187-189]. In addition to our example they also mention the cost problem that is usually a tradeoff between the nodes that are directly covered by circuits and the others. 


\subsection{Scheduling constraints}

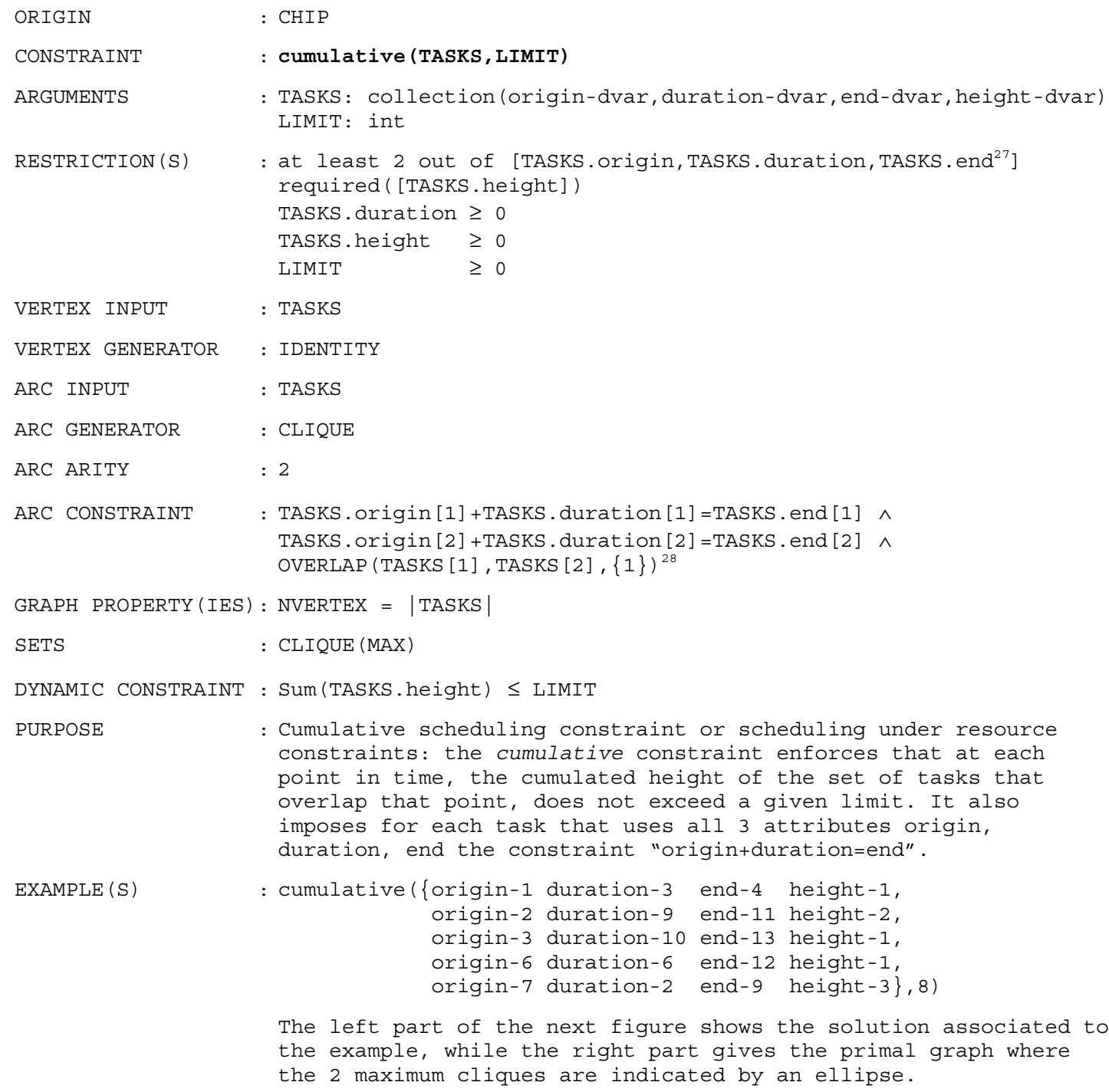

: Cumulative scheduling constraint or scheduling under resource constraints: the cumulative constraint enforces that at each point in time, the cumulated height of the set of tasks that overlap that point, does not exceed a given limit. It also imposes for each task that uses all 3 attributes origin, duration, end the constraint "origin+duration=end".

$\operatorname{EXAMPLE}(\mathrm{S})$

The left part of the next figure shows the solution associated to the example, while the right part gives the primal graph where the 2 maximum cliques are indicated by an ellipse.

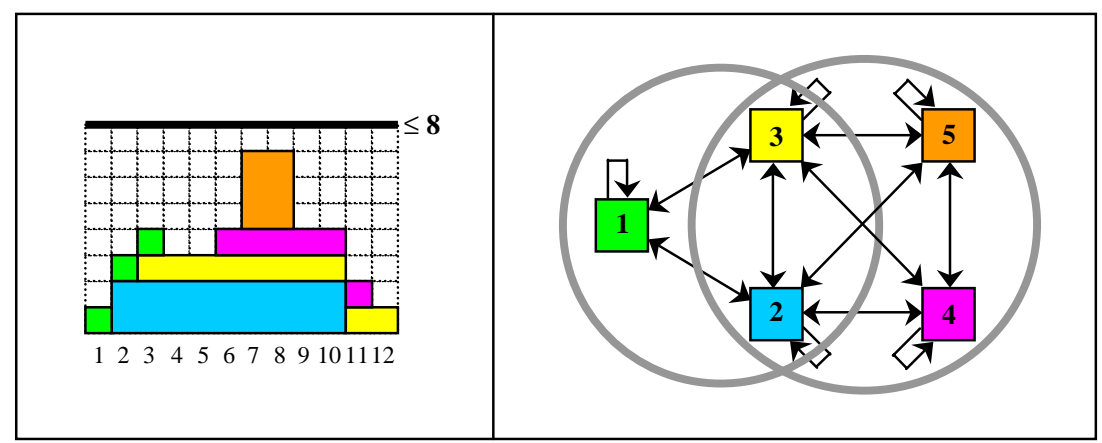

Figure 36. Cumulative solution and primal graph with its 2 maximum cliques.

COMMENT
Note that we integrate the "local" constraint that relates the origin, the duration and the end of each task within the arc constraint. In order to enforce the "local" constraints we use

\footnotetext{
${ }^{27}$ If the duration is not fixed and the end is more constrained than the origin then the end variable contains some information that you don't get when you consider only the origin and the duration variables.

${ }^{28}$ Refers to the overlapping constraint that was introduced in paragraph 2.2.1. . In our case we have only one single dimension.
} 
the graph property "NVERTEX = |TASKS $\mid$ " which states that the number of vertices of the primal graph should be equal to the number of tasks. If for a given task its "local" constraint does not hold then this makes false all the arc constraints attach to this task; as a consequence the vertex associated to that task would not occur any more in the primal graph and the graph property would not hold. 


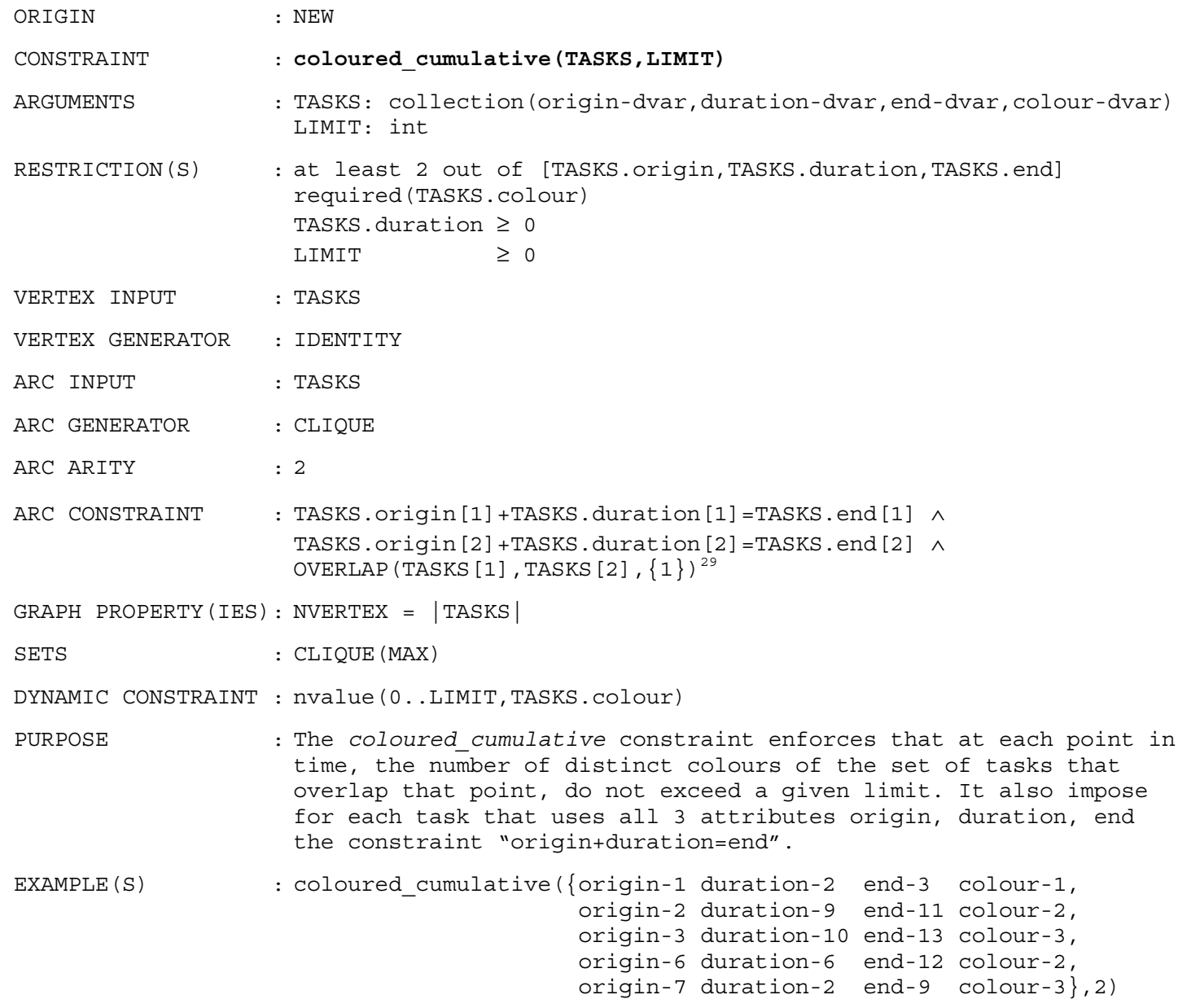

EXAMPLE (S)

: coloured_cumulative(\{origin-1 duration-2 end-3 colour-1, origin-2 duration-9 end-11 colour-2, origin-3 duration-10 end-13 colour-3, origin- 6 duration-6 end-12 colour-2, origin-7 duration-2 end-9 colour-3\},2)

The left part of the next figure shows the solution associated to the example, while the right part gives the primal graph where the 2 maximum cliques are enclosed by an ellipse.

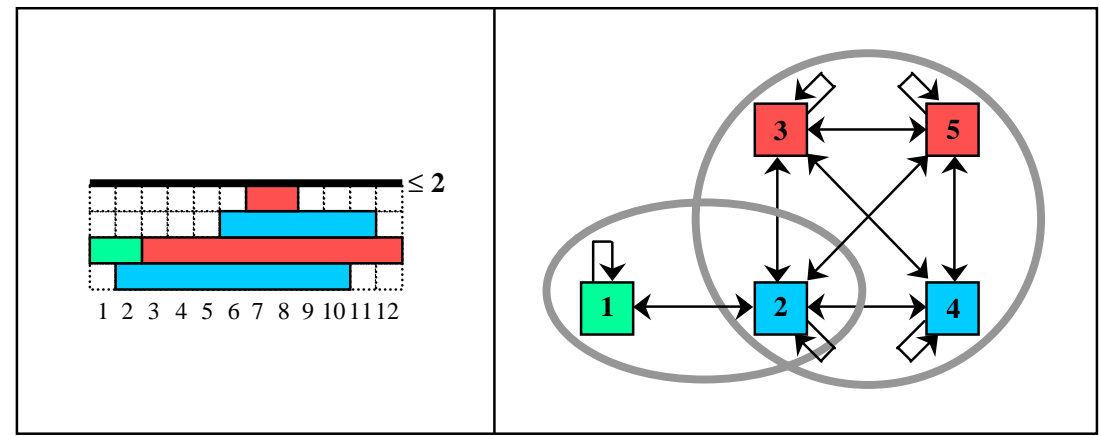

Figure 37. A coloured cumulative solution with at most 2 distinct colours in parallel and the related primal graph with its maximum cliques.

COMMENT

\begin{abstract}
: Note that we integrate the "local" constraint that relates the origin, the duration and the end of each task within the arc constraint. In order to enforce the "local" constraints we use the graph property "NVERTEX = |TASKS|" which states that the number of vertices of the primal graph should be equal to the number of tasks. If for a given task its "local" constraint does not hold then this makes false all the arc constraints attach to this task; as a consequence the vertex associated to that task
\end{abstract}

\footnotetext{
${ }^{29}$ Refers to the overlapping constraint that was introduced in paragraph 2.2.1. . In our case we have only one single dimension.
} 
would not occur any more in the primal graph and the graph property would not hold any more.

: Useful for scheduling problems where a machine can only proceed in parallel a maximum number of tasks of distinct type. 


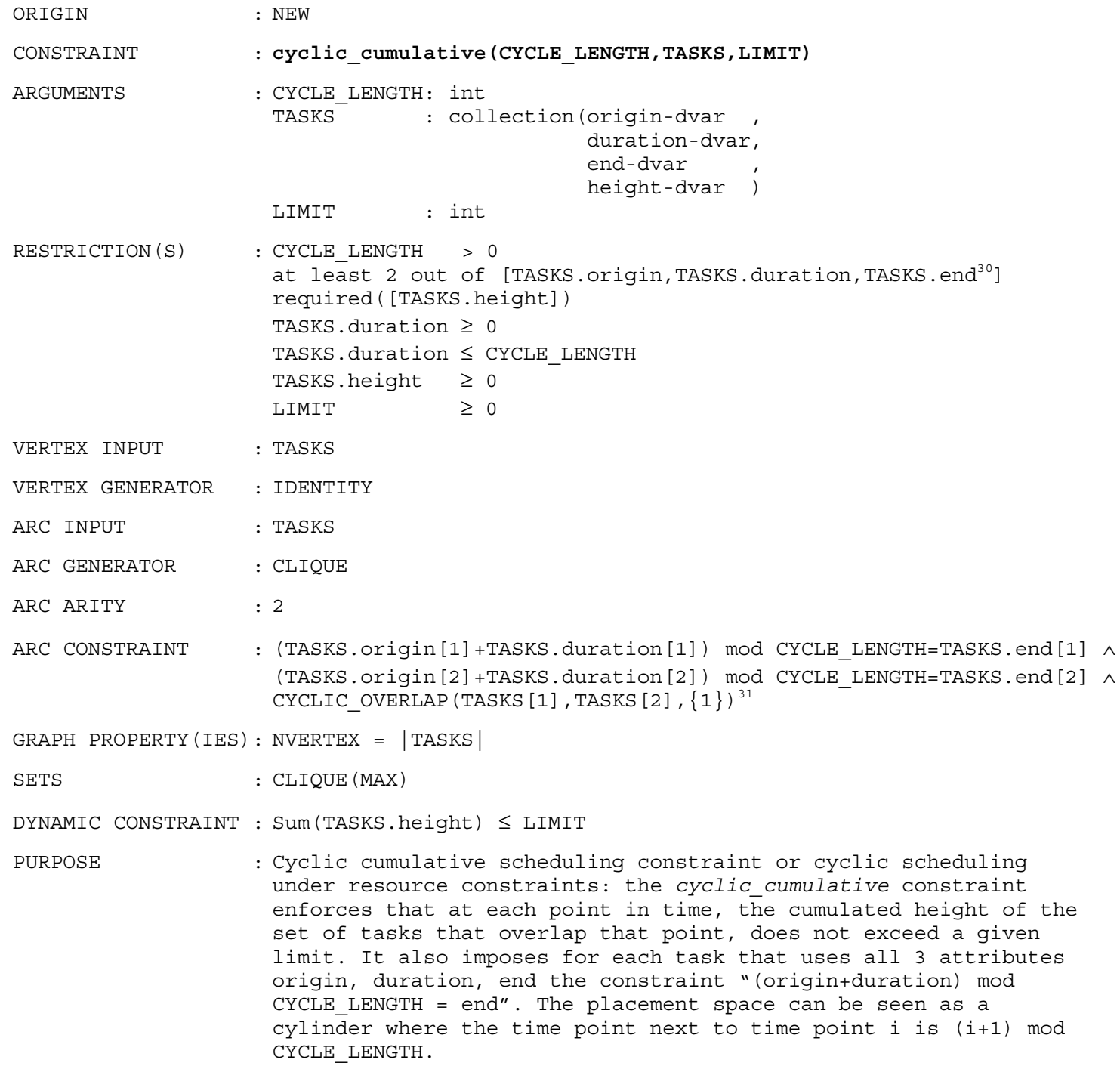

\footnotetext{
${ }^{30}$ If the duration is not fixed and the end is more constrained than the origin then the end variable contains some information that you don't get when you consider only the origin and the duration variables.

${ }^{31}$ Refers to the cyclic overlapping constraint that was introduced in paragraph 2.2.1. . In our case we have only one single dimension.
} 

origin-1 duration-9 end-10 height-2, origin-5 duration-10 end-3 height-1, origin-5 duration-7 end-0 height-1, origin-6 duration-2 end-8 height-4\},8)

The left part of the next figure shows the solution associated to the example, while the right part gives the primal graph where the 2 maximum cliques are indicated by an ellipse.

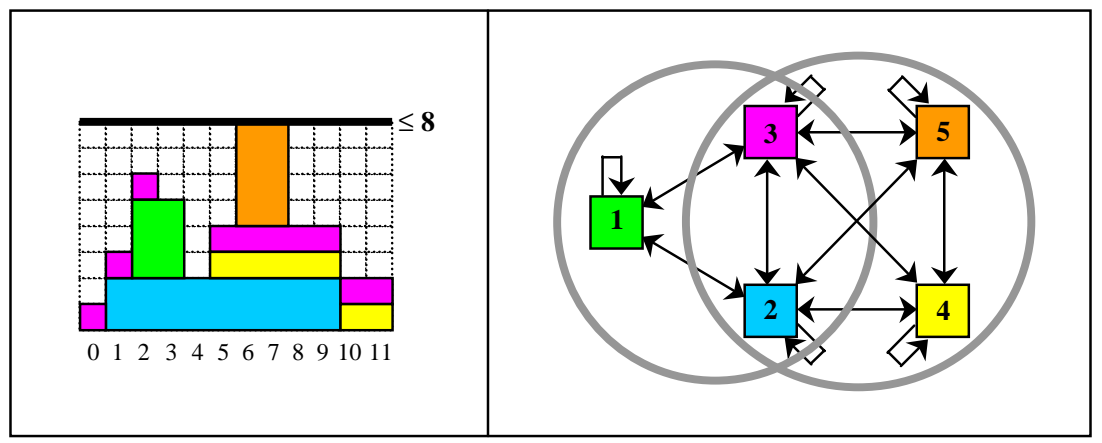

Figure 38. Cyclic cumulative solution and primal graph with its 2 maximum cliques.

: Note that we integrate the "local" constraint that relates the origin, the duration and the end of each task within the arc constraint. In order to enforce the "local" constraints we use the graph property "NVERTEX = |TASKS $\mid$ " which states that the number of vertices of the primal graph should be equal to the number of tasks. If for a given task its "local" constraint does not hold then this makes false all the arc constraints attach to this task; as a consequence the vertex associated to that task would not occur any more in the primal graph and the graph property would not hold. 


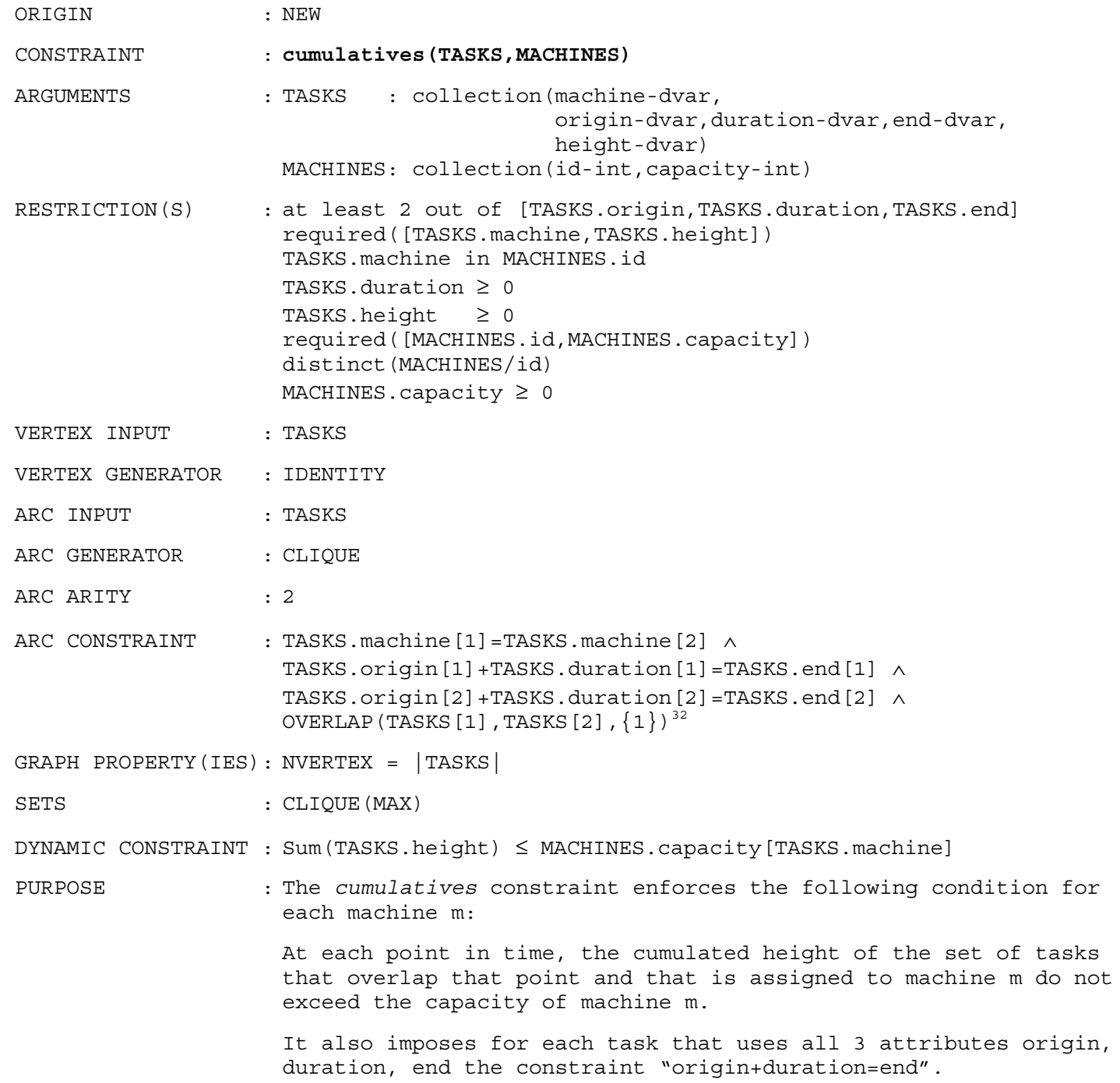
${ }^{32}$ Refers to the overlapping constraint that was introduced in paragraph 2.2.1. . In our case we have only one
single dimension. 
The left part of the next figure shows the solution associated to the example, while the right part gives the primal graph where the 3 maximum cliques are enclosed by an ellipse.

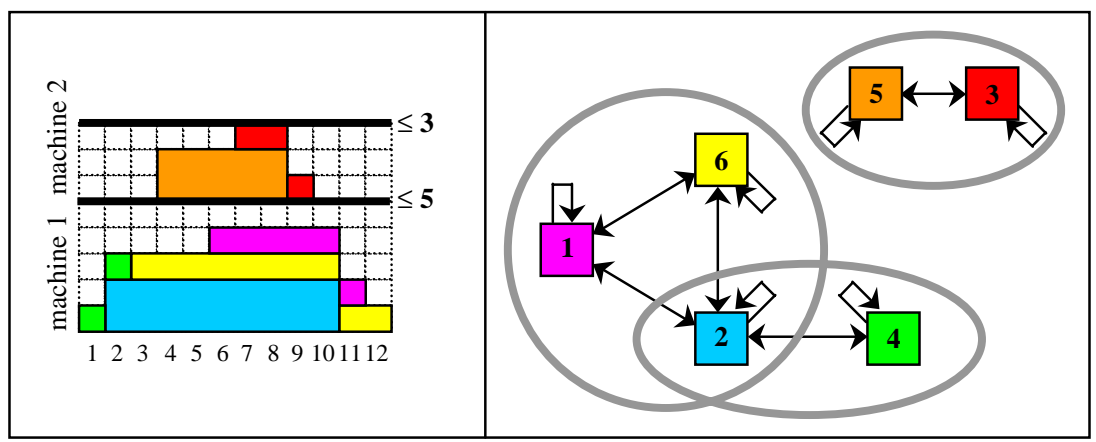

Figure 39. A cumulative with machine-assignment solution and the related primal graph with its 3 maximum cliques.

: Note that we integrate the "local" constraint that relates the origin, the duration and the end of each task within the arc constraint. In order to enforce the "local" constraints we use the graph property "NVERTEX = |TASKS|" which states that the number of vertices of the primal graph should be equal to the number of tasks. If for a given task its "local" constraint does not hold then this makes false all the arc constraints attach to this task; as a consequence the vertex associated to that task would not occur any more in the primal graph and the graph property would not hold any more.

: Useful for scheduling problems where several "cumulative" machines are available and where you have to assign each task on a specific machine. 


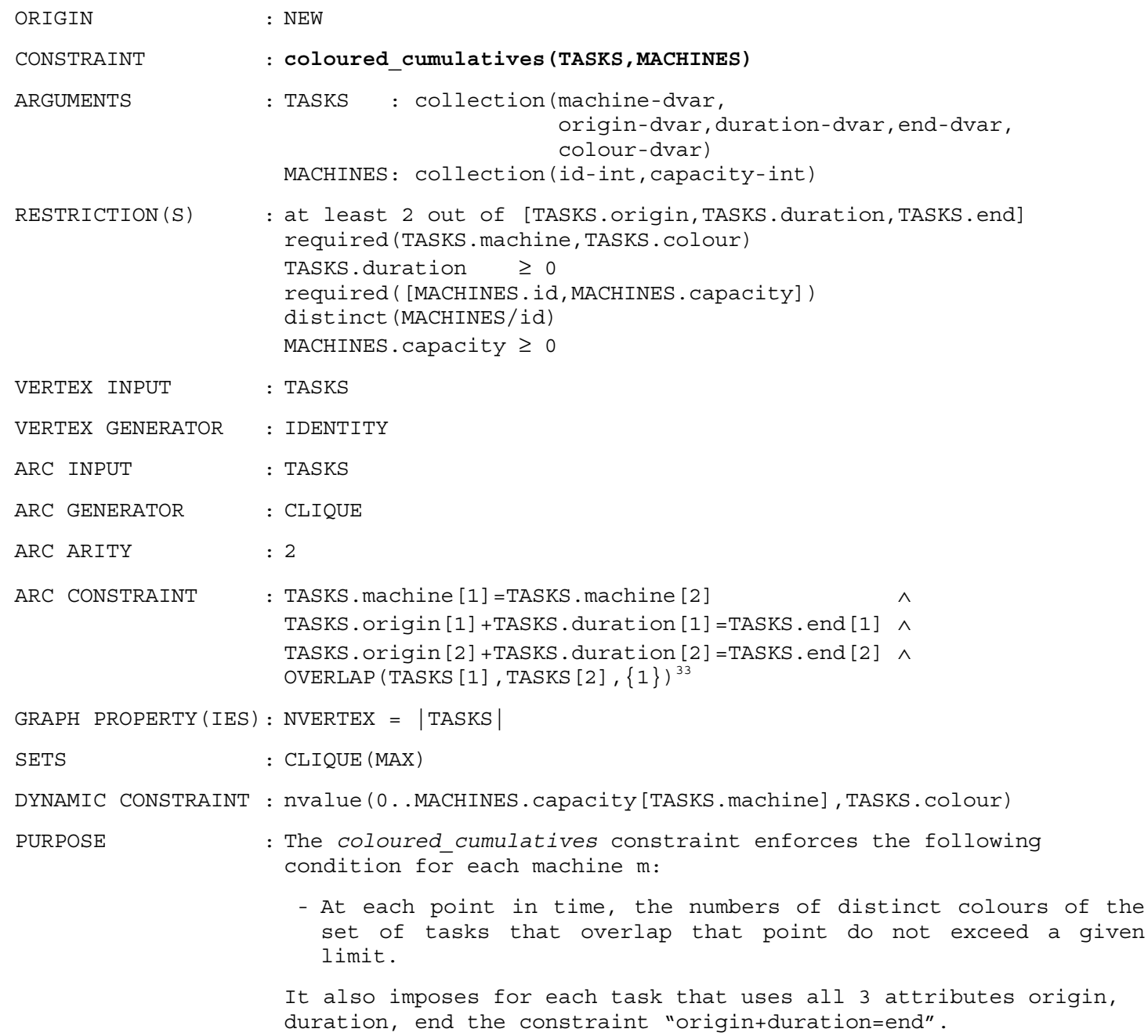

\footnotetext{
${ }^{33}$ Refers to the overlapping constraint that was introduced in paragraph 2.2.1. . In our case we have only one
} single dimension. 


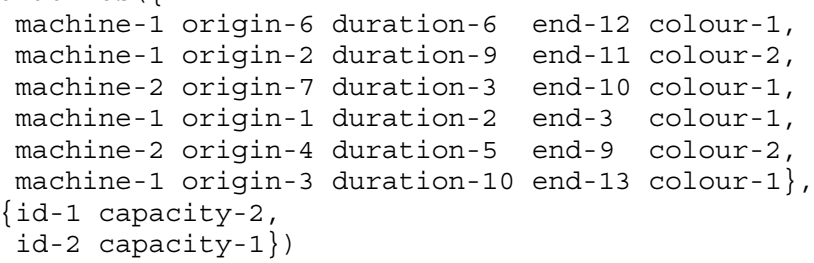

The left part of the next figure shows the solution associated to the example, while the right part gives the primal graph where the 3 maximum cliques are enclosed by an ellipse. For each of the 2 cliques that are associated to machine 1 we have only 2 distinct colours, while for the unique clique of machine 2 we have one single colour. Since this match the respective capacities of the 2 machines, the constraint hold.

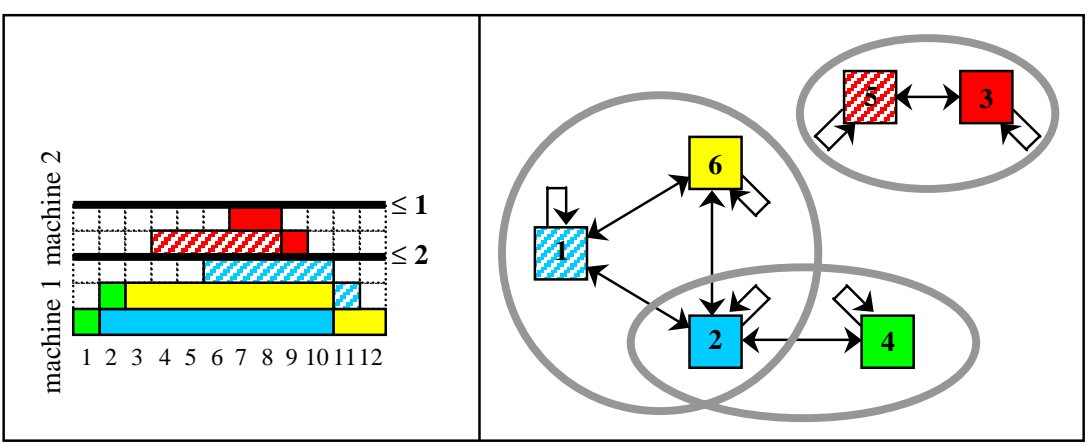

Figure 40. A coloured cumulative with machine-assignment solution and the related primal graph with its 3 maximum cliques.

: Note that we integrate the "local" constraint that relates the origin, the duration and the end of each task within the arc constraint. In order to enforce the "local" constraints we use the graph property "NVERTEX = |TASKS|" which states that the number of vertices of the primal graph should be equal to the number of tasks. If for a given task its "local" constraint does not hold then this makes false all the arc constraints attach to this task; as a consequence the vertex associated to that task would not occur any more in the primal graph and the graph property would not hold any more.

: Useful for scheduling problems where several "coloured cumulative" machines are available and where you have to assign each task on a specific machine. 


\subsection{Geometrical constraints}

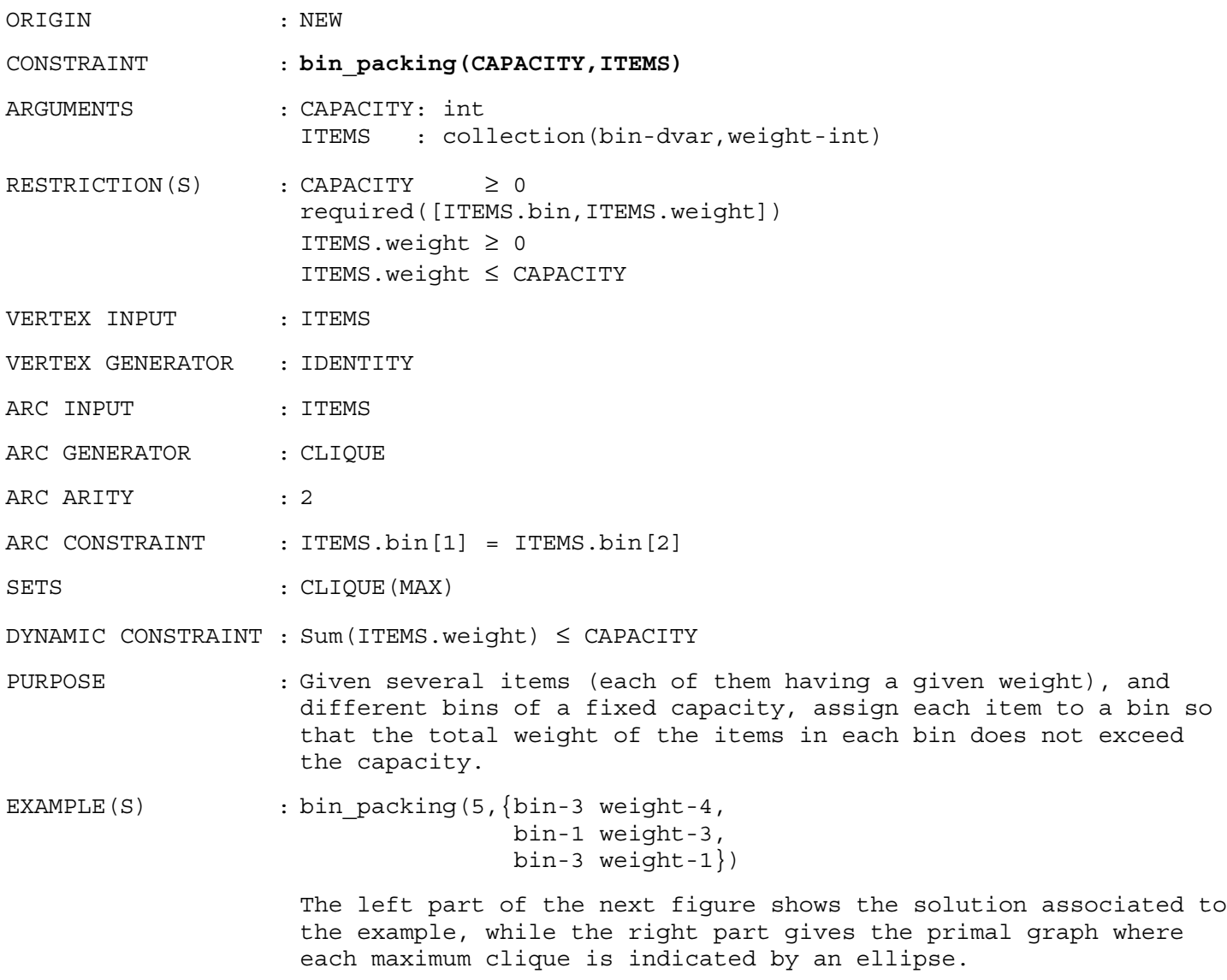

EXAMPLE (S)

: bin_packing $(5,\{$ bin-3 weight -4 ,

bin-1 weight-3,

bin-3 weight-1\})

The left part of the next figure shows the solution associated to the example, while the right part gives the primal graph where each maximum clique is indicated by an ellipse.

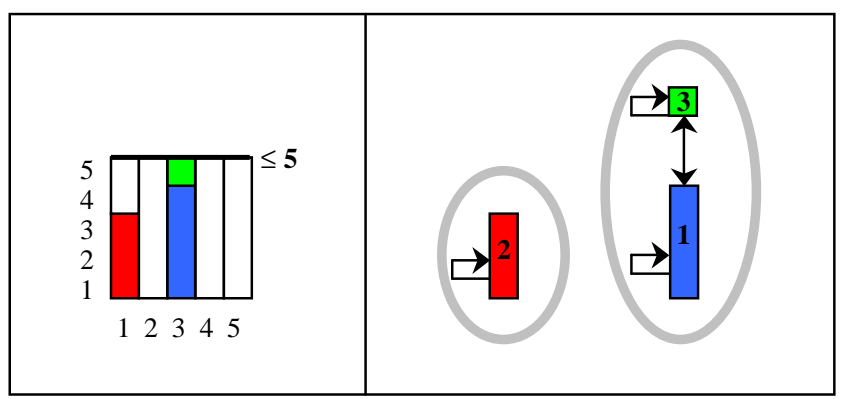

Figure 41. Bin-packing solution and related primal graph with its 2 maximum cliques.

COMMENT

: We enforce the n-ary sum constraint ${ }^{34}$ on the weight of the items that belong to a same maximum clique. Since we use the equality constraint, the maximum cliques correspond to all items that are assigned to the same bin.

REMARK

: Note the difference with the "classical" bin-packing problem [MARTELLO \& TOTH 90, page 221] where one wants to find solutions that minimize the number of bins. In our case each item may be assigned only to specific bins (i.e. the different values of the bin variable) and the goal is to find a feasible solution.

This constraint can be seen as a special case of the cumulative constraint [AGGOUN \& BELDICEANU 93], where all tasks durations are equal to 1 .

\footnotetext{
${ }^{34}$ Refers to the Sum constraint that was introduced in the paragraph 2.2.1. . It enforces the sum of variables to be between 2 given limits.
} 


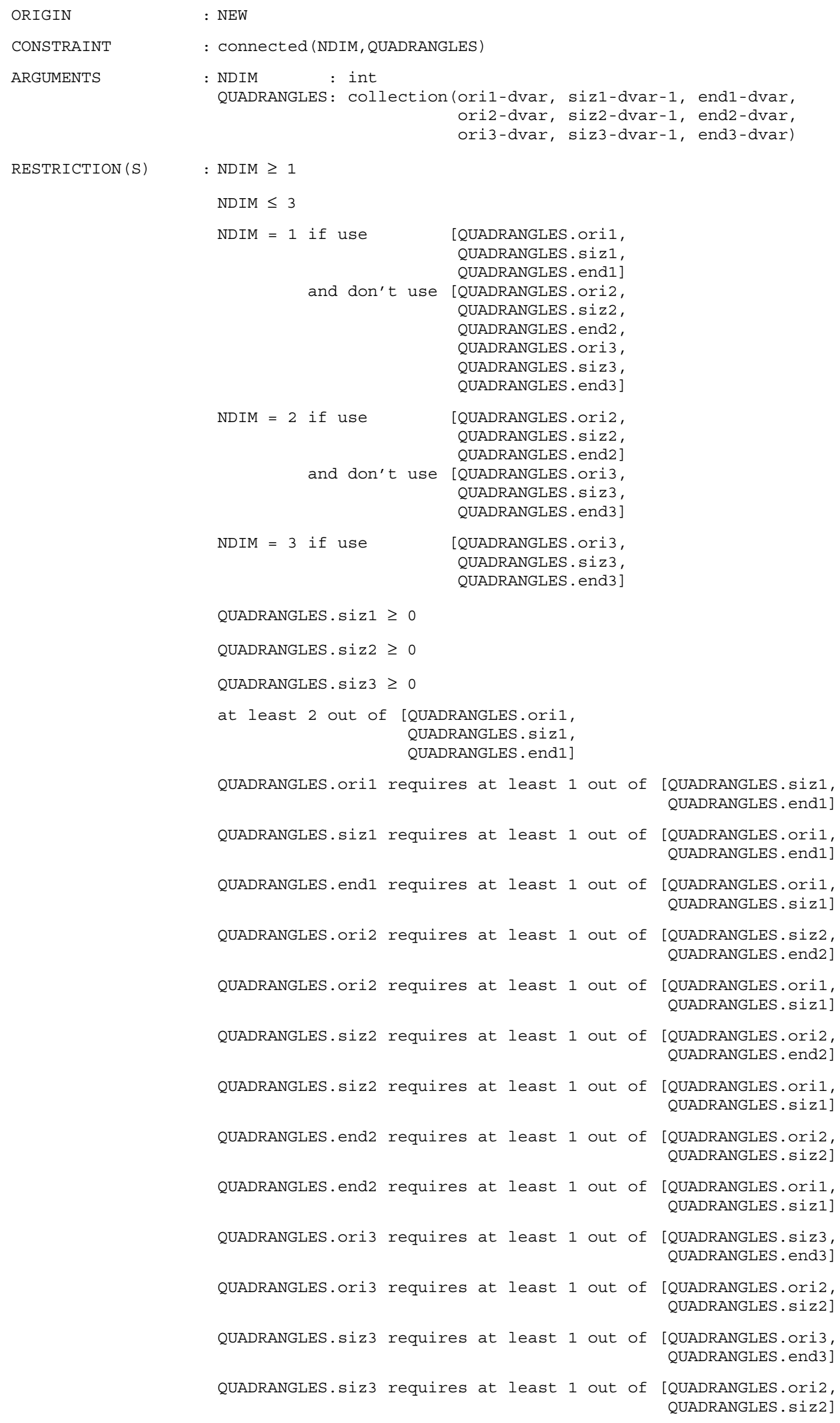


QUADRANGLES.end3 requires at least 1 out of [QUADRANGLES.ori3, QUADRANGLES.Siz3]

QUADRANGLES.end3 requires at least 1 out of [QUADRANGLES.ori2, QUADRANGLES . siz2]

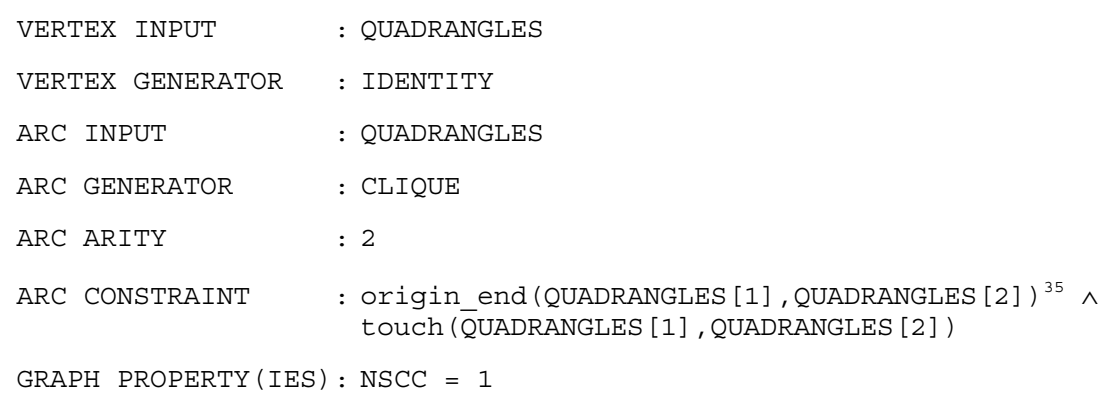

$\operatorname{EXAMPLE}(\mathrm{S})$

: There should be one single group of connected quadrangles. Two quadrangles touch each other if they overlap in all dimensions except one, and if for the dimension where they do not overlap the distance between the 2 quadrangles is equal to 0 .

connected(2, \{oril-2 siz1-4 end1-6 ori2-2 siz2-2 end2-4, oril-1 siz1-2 end1-3 ori2-4 siz2-3 end2-7, oril-7 siz1-4 end1-11 ori2-1 siz2-2 end2-3, oril-6 sizl-2 end1-8 ori2-3 siz2-2 end2-5\})

The next figure shows the rectangles associated to the example. One can observe that:

- Rectangle 2 touch rectangle 1 ,

- Rectangle 1 touch rectangle 2 and rectangle 4,

- Rectangle 4 touch rectangle 1 and rectangle 3 ,

- Rectangle 3 touch rectangle 4 .

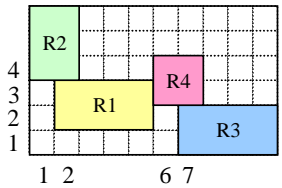

Figure 42. Four connected rectangles.

USAGE

: In floor planning problem there is a constraint, which states that one should be able to access every room from any room.

\footnotetext{
${ }^{35}$ This elementary constraint was defined in paragraph 2.2.2. . Its purpose is to enforce all the constraints of the
} form "origin+size=end" that link the attributes of the 2 quadrangles. 


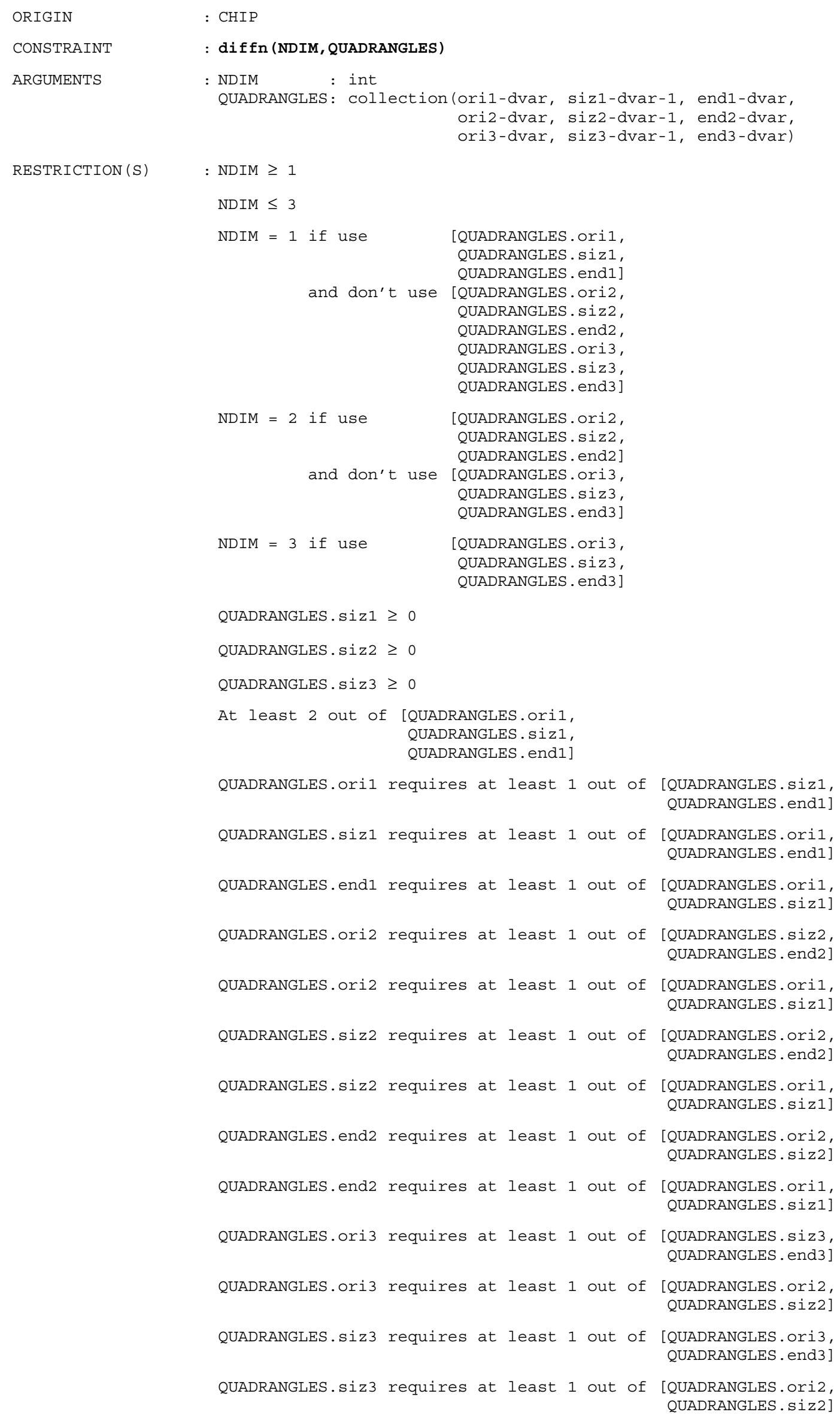


QUADRANGLES.end3 requires at least 1 out of [QUADRANGLES.ori3, QUADRANGLES. siz3]

QUADRANGLES.end3 requires at least 1 out of [QUADRANGLES.ori2, QUADRANGLES . siz2]

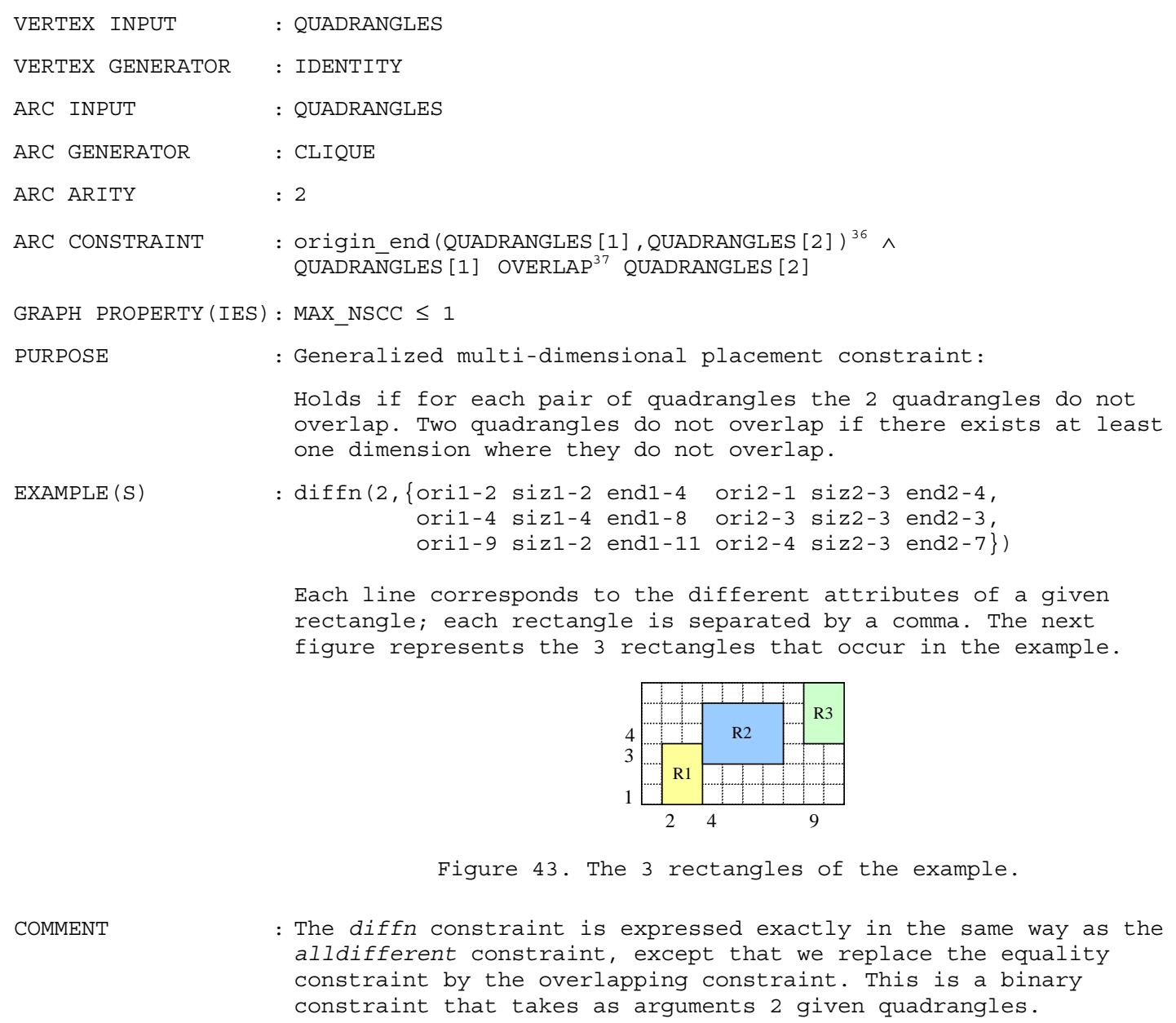

$\operatorname{EXAMPLE~(S)~}$

: diffn(2,\{oril-2 siz1-2 end1-4 ori2-1 siz2-3 end2-4, oril-4 siz1-4 end1-8 ori2-3 siz2-3 end2-3, oril-9 sizl-2 end1-11 ori2-4 siz2-3 end2-7\})

Each line corresponds to the different attributes of a given rectangle; each rectangle is separated by a comma. The next figure represents the 3 rectangles that occur in the example.

Figure 43. The 3 rectangles of the example.

COMMENT

\begin{abstract}
: The diffn constraint is expressed exactly in the same way as the alldifferent constraint, except that we replace the equality constraint by the overlapping constraint. This is a binary constraint that takes as arguments 2 given quadrangles.
\end{abstract}

\footnotetext{
${ }^{36}$ This elementary constraint was defined in paragraph 2.2 .2 . . Its purpose is to enforce all the constraints of the form "origin+size=end" that link the attributes of the 2 quadrangles.

${ }^{37}$ The binary constraint OVERLAP between $2 n$-dimensional quadrangles holds if the 2 quadrangles overlap in all dimensions.
} 


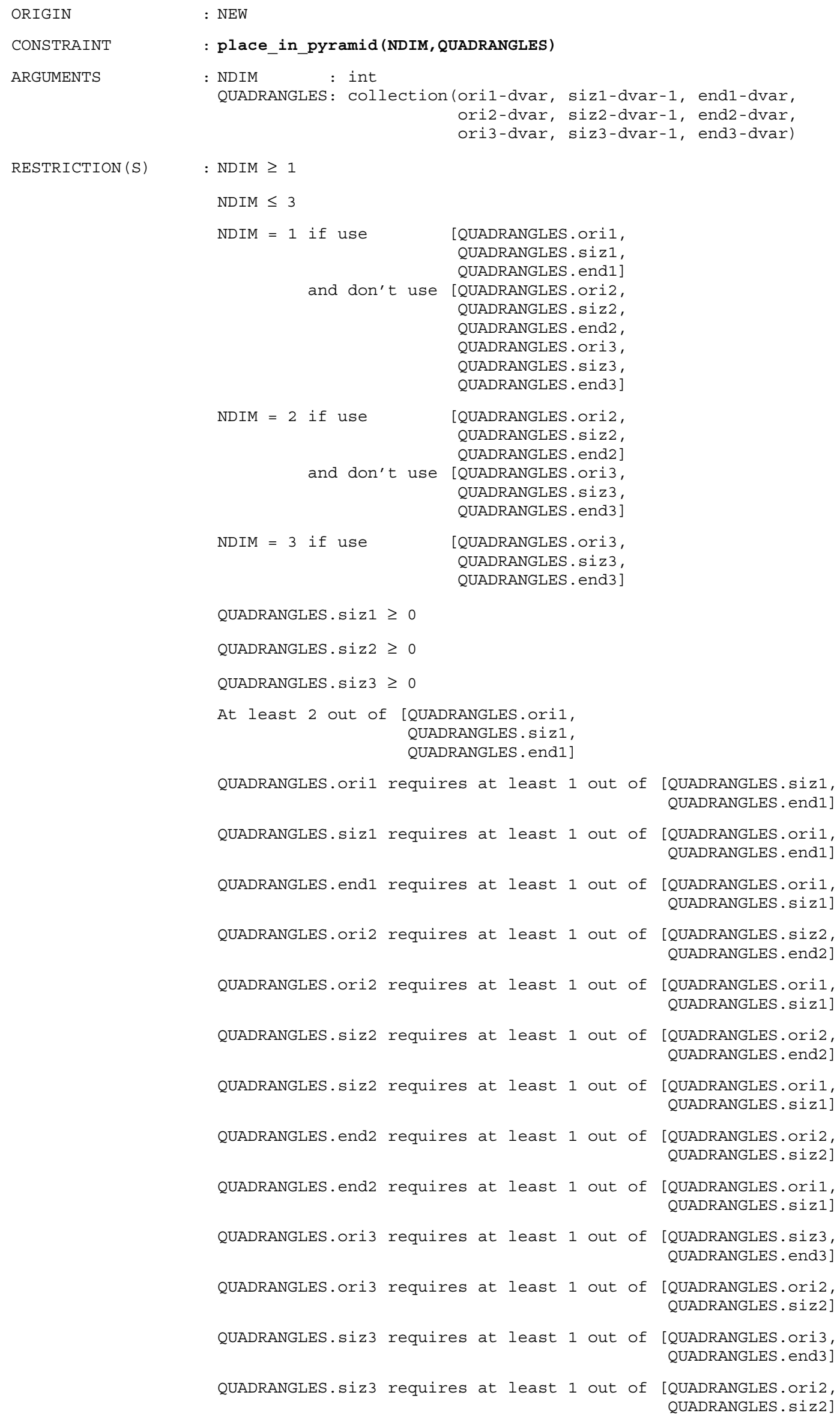


QUADRANGLES.end3 requires at least 1 out of [QUADRANGLES.ori3, QUADRANGLES. siz3]

QUADRANGLES.end3 requires at least 1 out of [QUADRANGLES.ori2, QUADRANGLES . siz2]

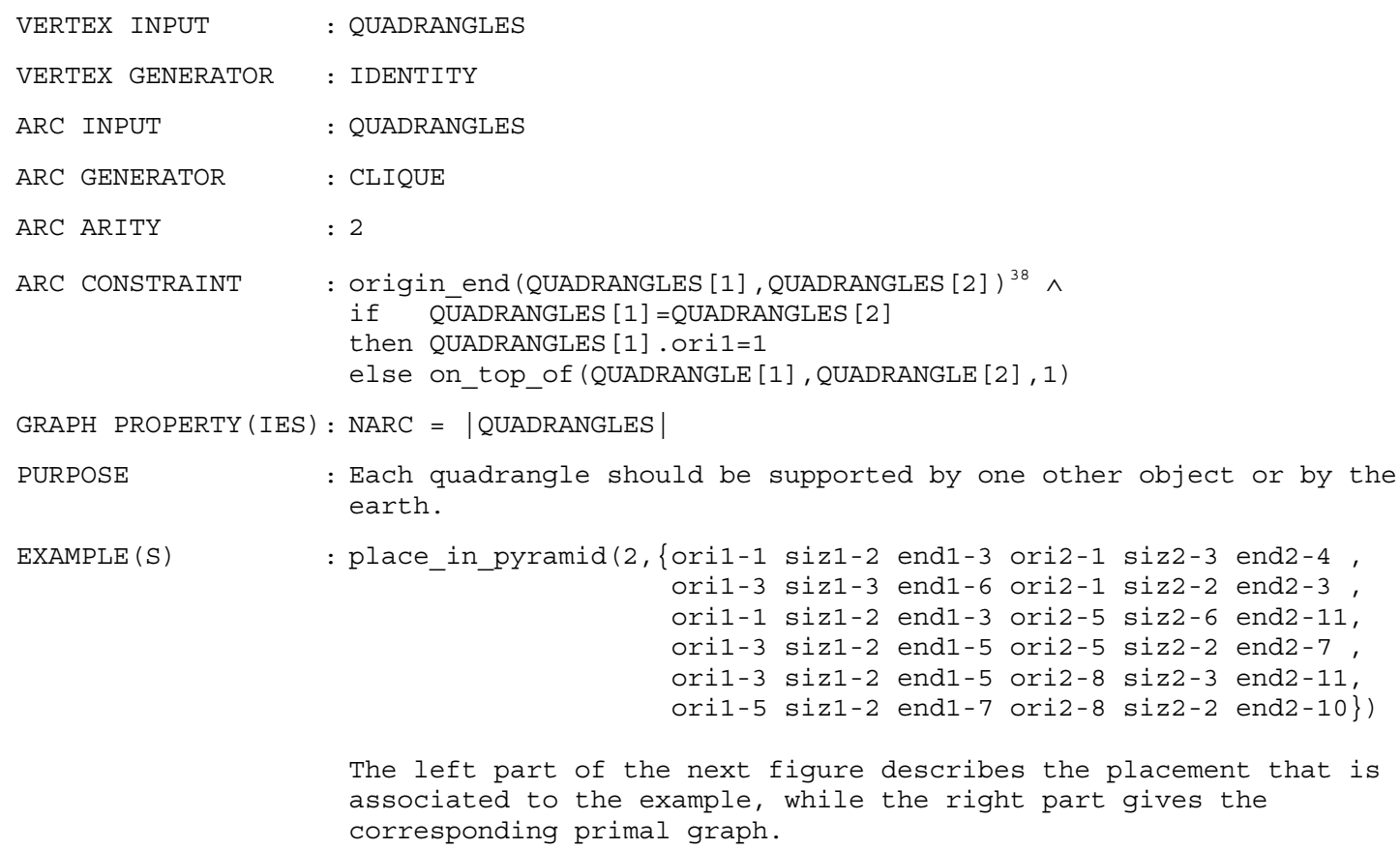

EXAMPLE ( S)

:place_in_pyramid(2,\{ori1-1 siz1-2 end1-3 ori2-1 siz2-3 end2-4, oril-3 siz1-3 end1-6 ori2-1 siz2-2 end2-3, ori1-1 siz1-2 end1-3 ori2-5 siz2-6 end2-11, oril-3 siz1-2 end1-5 ori2-5 siz2-2 end2-7, ori1-3 siz1-2 end1-5 ori2-8 siz2-3 end2-11, ori1-5 siz1-2 end1-7 ori2-8 siz2-2 end2-10\})

The left part of the next figure describes the placement that is associated to the example, while the right part gives the corresponding primal graph.
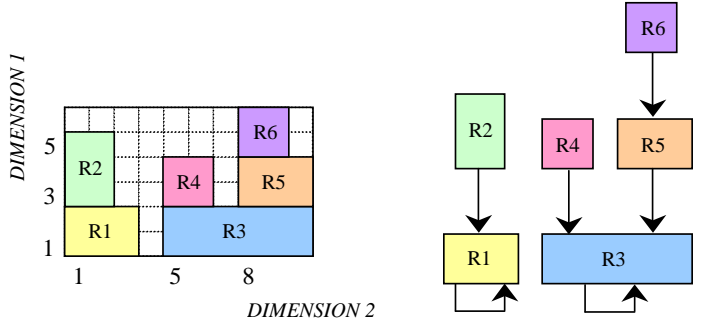

Figure 44. Rectangles and primal graph.

COMMENT

: The arc constraint enforces one of the following constraints:

- If the arc connects the same object then the constraint corresponds to the fact that the earth directly supports the object,

- If the arc connects 2 distinct objects then the constraint is: one object is on top of the other object (i.e. in all dimensions except dimension 2 it is include, in dimension 2 it is after).

USAGE

: The diffn ${ }^{39}$ constraint is not enough if one wants to produce a placement where no object "floats" in the air. This constraint is usually handled with a heuristic during the enumeration.

\footnotetext{
${ }^{38}$ This elementary constraint was defined in paragraph 2.2.2. . Its purpose is to enforce all the constraints of the form "origin+size=end" that link the attributes of the 2 quadrangles.

${ }^{39}$ The diffn constraint enforces that all the quadrangles from a collection of quadrangles to not pairwise overlap.
} 


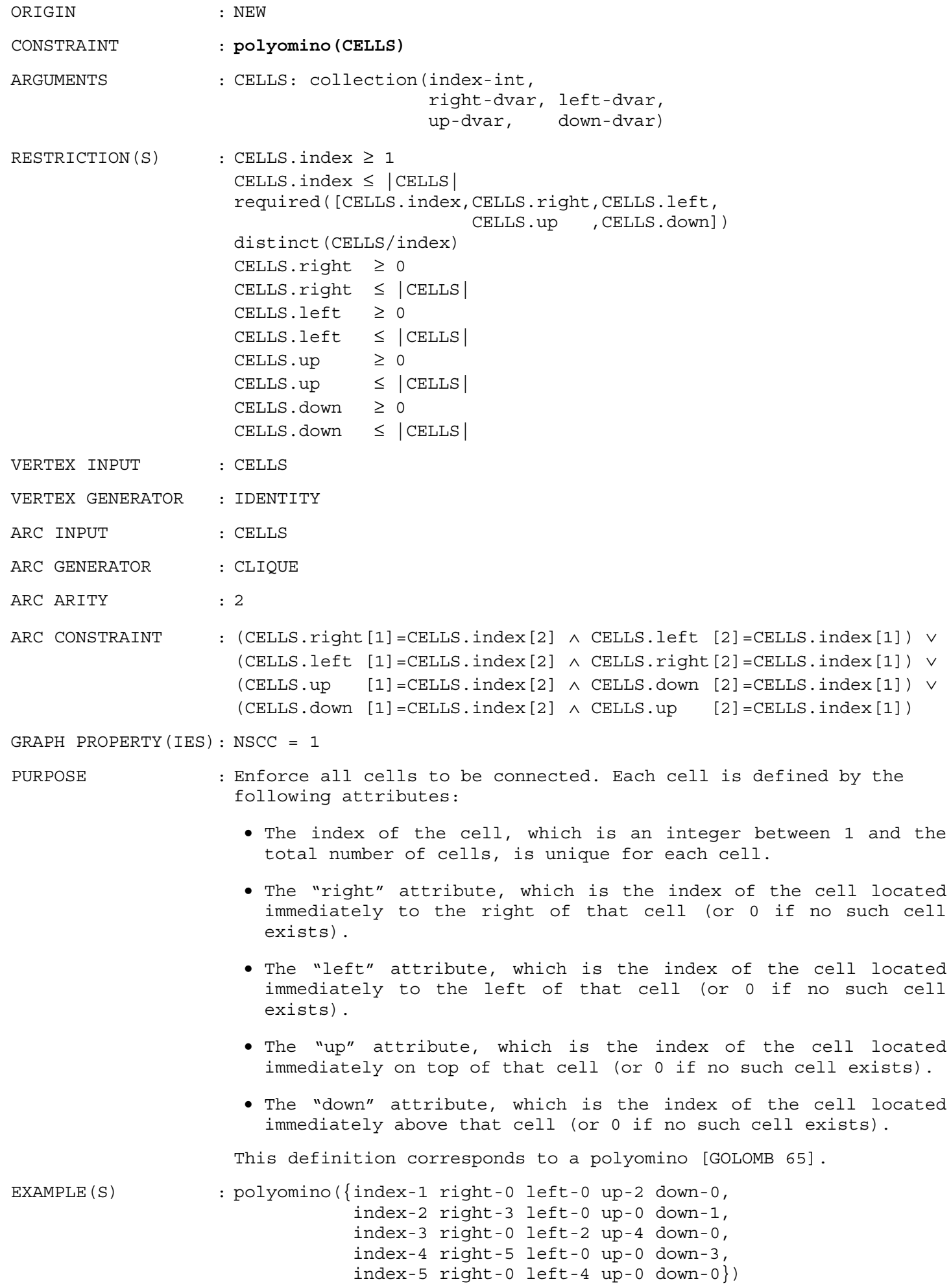

- The index of the cell, which is an integer between 1 and the total number of cells, is unique for each cell.

- The "right" attribute, which is the index of the cell located immediately to the right of that cell (or 0 if no such cell exists).

- The "left" attribute, which is the index of the cell located immediately to the left of that cell (or 0 if no such cell exists).

- The "up" attribute, which is the index of the cell located immediately on top of that cell (or 0 if no such cell exists).

- The "down" attribute, which is the index of the cell located immediately above that cell (or 0 if no such cell exists).

This definition corresponds to a polyomino [GOLOMB 65].

EXAMPLE (S)

: polyomino( $\{$ index-1 right-0 left-0 up-2 down-0, index-2 right-3 left-0 up-0 down-1, index-3 right-0 left-2 up-4 down-0, index-4 right-5 left-0 up-0 down-3, index-5 right-0 left-4 up-0 down-0\}) 
The next figure shows the polyomino associated to the example.

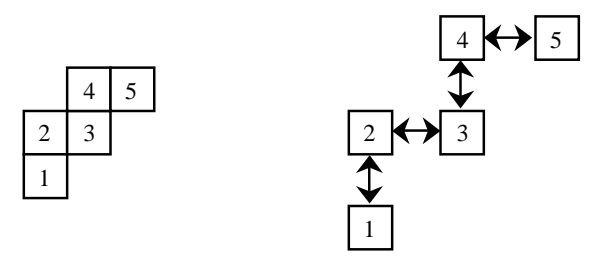

Figure 45. A polyomino and its primal graph. 


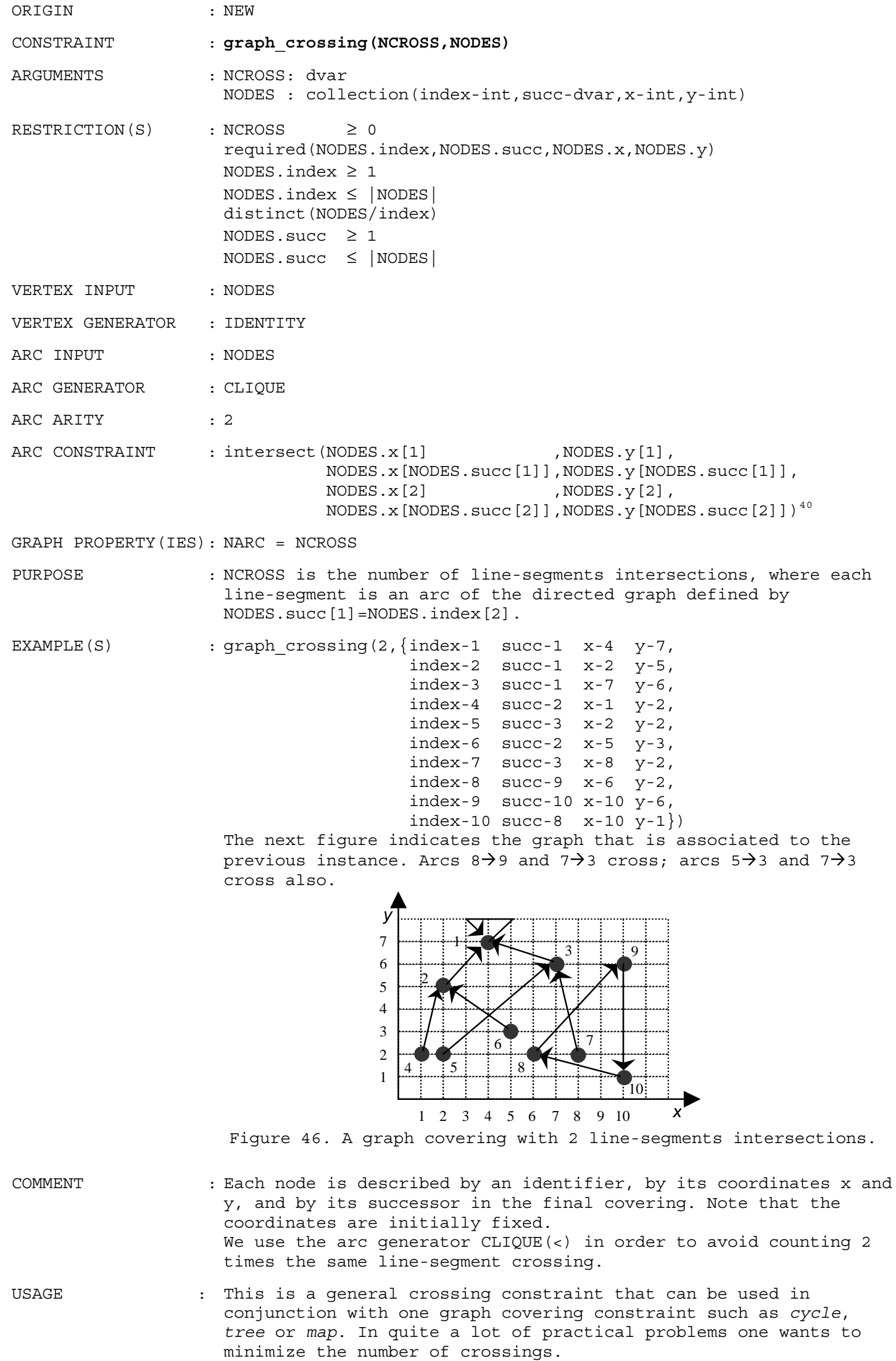

EXAMPLE (S)

: graph_crossing $(2,\{$ index-1 succ-1 $x-4 \quad y-7$,

index-2 succ-1 $x-2 \quad y-5$,

index-3 succ-1 $x-7 \quad y-6$,

index-4 succ-2 $\mathrm{x}-1$ y-2,

index-5 succ-3 $x-2 \quad y-2$,

index-6 succ-2 $x-5 \quad y-3$,

index-7 succ-3 $x-8 \quad y-2$,

index-8 succ-9 $x-6 \quad y-2$,

index-9 succ-10 $x-10 \quad y-6$

index-10 succ-8 $\mathrm{x}-10 \mathrm{y}-1\}$ )

The next figure indicates the graph that is associated to the previous instance. Arcs $8 \rightarrow 9$ and $7 \rightarrow 3$ cross; arcs $5 \rightarrow 3$ and $7 \rightarrow 3$ cross also.

Figure 46. A graph covering with 2 line-segments intersections.

COMMENT

: Each node is described by an identifier, by its coordinates $x$ and $y$, and by its successor in the final covering. Note that the coordinates are initially fixed.

We use the arc generator CLIQUE $(<)$ in order to avoid counting 2 times the same line-segment crossing.

USAGE

: This is a general crossing constraint that can be used in conjunction with one graph covering constraint such as cycle, tree or map. In quite a lot of practical problems one wants to minimize the number of crossings.

\footnotetext{
40 intersect(x_start1,y_start1,x_end1,y_end1,x_start2,y_start2,x_end2,y_end2) holds if the 2 line-segments intersect. One can find an implementation of the ground case in [SEDGEWICK 88 pages 350-351].
} 
: We could have also give one crossing constraint for each graph covering constraint, but we feel that it is better to start first with a more general constraint before going in the specificity of the pattern that is used for covering the graph. 


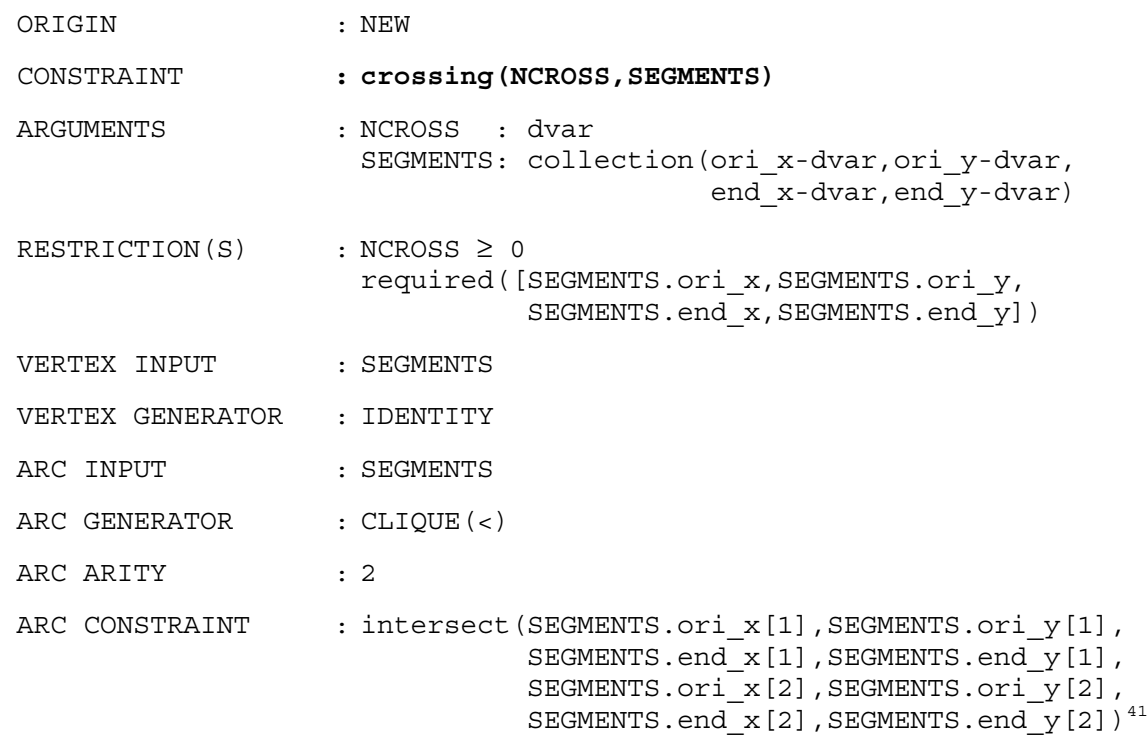

GRAPH PROPERTY (IES) : NARC = NCROSS

PURPOSE

: NCROSS is the number of line-segments intersections.

EXAMPLE (S)

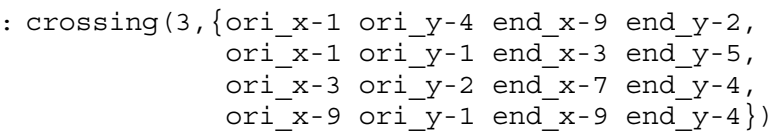

The next figure give a picture of the previous example, where one can observe the 3 line-segments intersections.

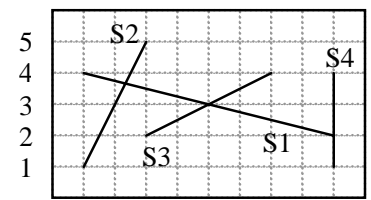

$\begin{array}{lllllllll}1 & 2 & 3 & 4 & 5 & 6 & 7 & 8 & 9\end{array}$

Figure 47. Intersection between line-segments.

COMMENT

\footnotetext{
: Each line-segment is described by the $\mathrm{x}$ and $\mathrm{y}$ coordinates of its 2 extremities.

In the arc generator we use the restriction < in order to generate one single arc for each pair of segments. This is required, since otherwise we would count more than once a linesegments intersection.
}

\footnotetext{
${ }^{41}$ intersect(x_start1,y_start1,x_end1,y_end1,x_start2,y_start2,x_end2,y_end2) holds if the 2 line-segments intersect.
} 


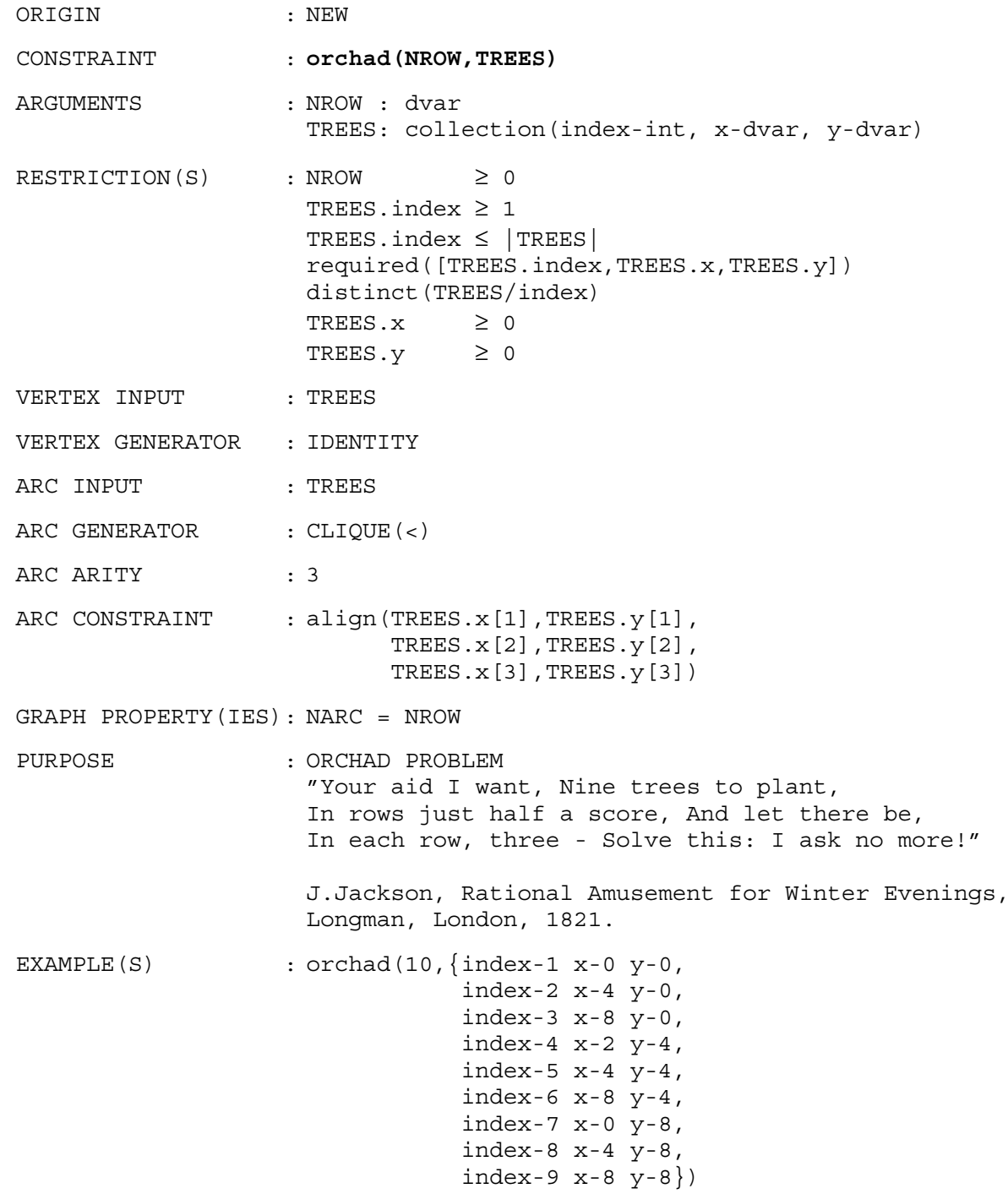

EXAMPLE (S)

The 10 alignments of 3 trees correspond to the following triples of trees: $(1,2,3),(1,4,8),(1,5,9),(2,4,7),(2,5,8),(2,6,9)$, $(3,5,7),(3,6,8),(4,5,6),(7,8,9)$. The figure below shows the 9 trees and the 10 alignments.

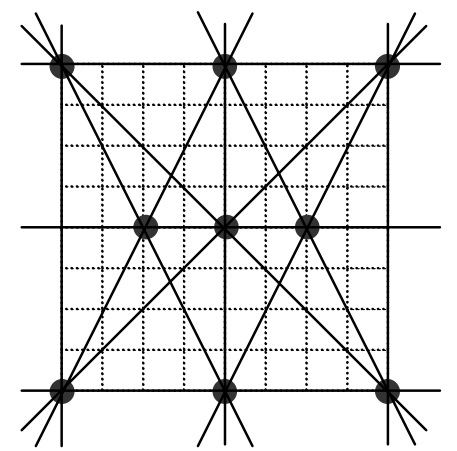

Figure 48. Nine trees with 10 alignments of 3 trees. to generate all the arcs of the directed hypergraph. Each arc is an ordered triple of trees. We use the restriction < in order to generate one single arc for each set of 3 trees. This is required, since otherwise we would count more than once a given alignment of 3 trees. 


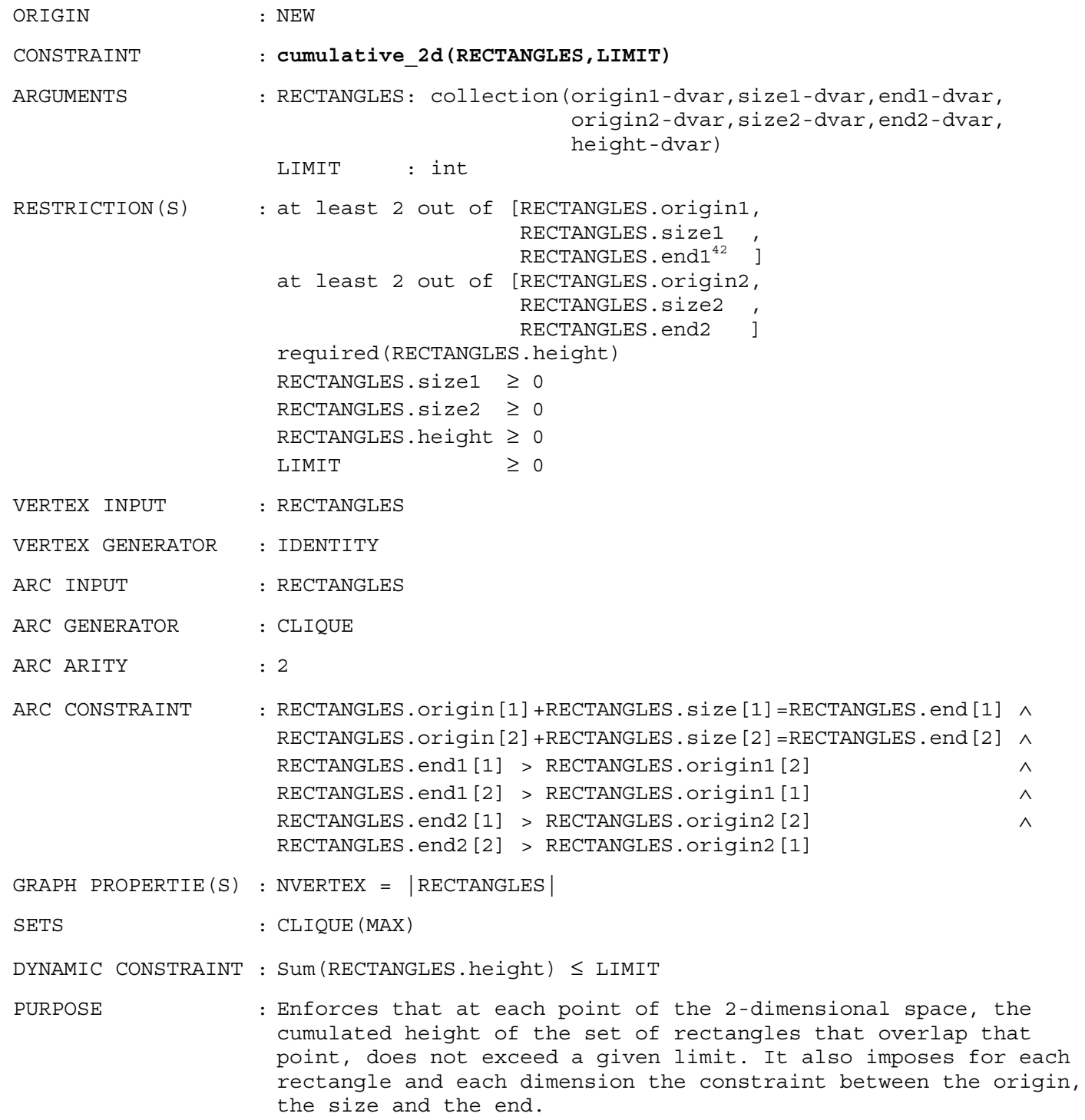
${ }^{42}$ If the size is not fixed and the end is more constrained than the origin then the end variable contains some
information that you don't get when you consider only the origin and the size variables. 
- cumulative $2 \mathrm{~d}($

\{origin1-1 size1-4 end1-5 origin2-3 size2-3 end2-6 height-4, origin1-3 size1-2 end1-5 origin2-1 size2-2 end2-3 height-2, origin1-1 size1-2 end1-3 origin2-1 size2-2 end2-3 height-3, origin1-4 size1-1 end1-5 origin2-1 size2-1 end2-2 height-1\}, 4)

Part (A) of next figure shows 4 rectangles of height $4,2,3$ and 1. Part (B) gives the corresponding cumulated 2-dimensional profile, where each number is the cumulated height of all the rectangles that contain the corresponding region.

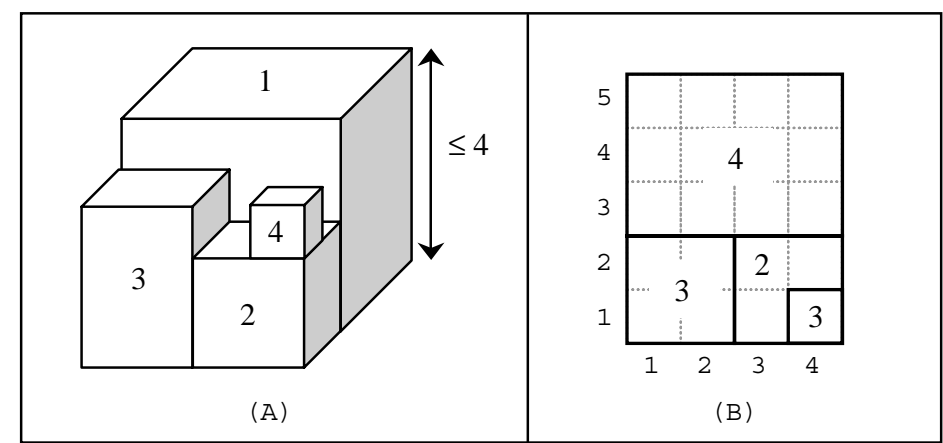

Figure 49. Two representations of a 2-dimensional cumulated profile.

: The cumulative_2d constraint is a necessary condition for the diffn constraint in 3 dimensions (i.e. the placement of parallelepipeds in such a way that they do not pairwise overlap and that each parallelepiped has his sides parallel to the sides of the placement space).

A first natural way to handle this constraint would be to accumulate the compulsory parts [LAHRICHI 82] of the rectangles in a quadtree [SAMET 89] for which each sub region has the cumulated height of the rectangles that contain that region. 


\subsection{Miscellaneous constraints}

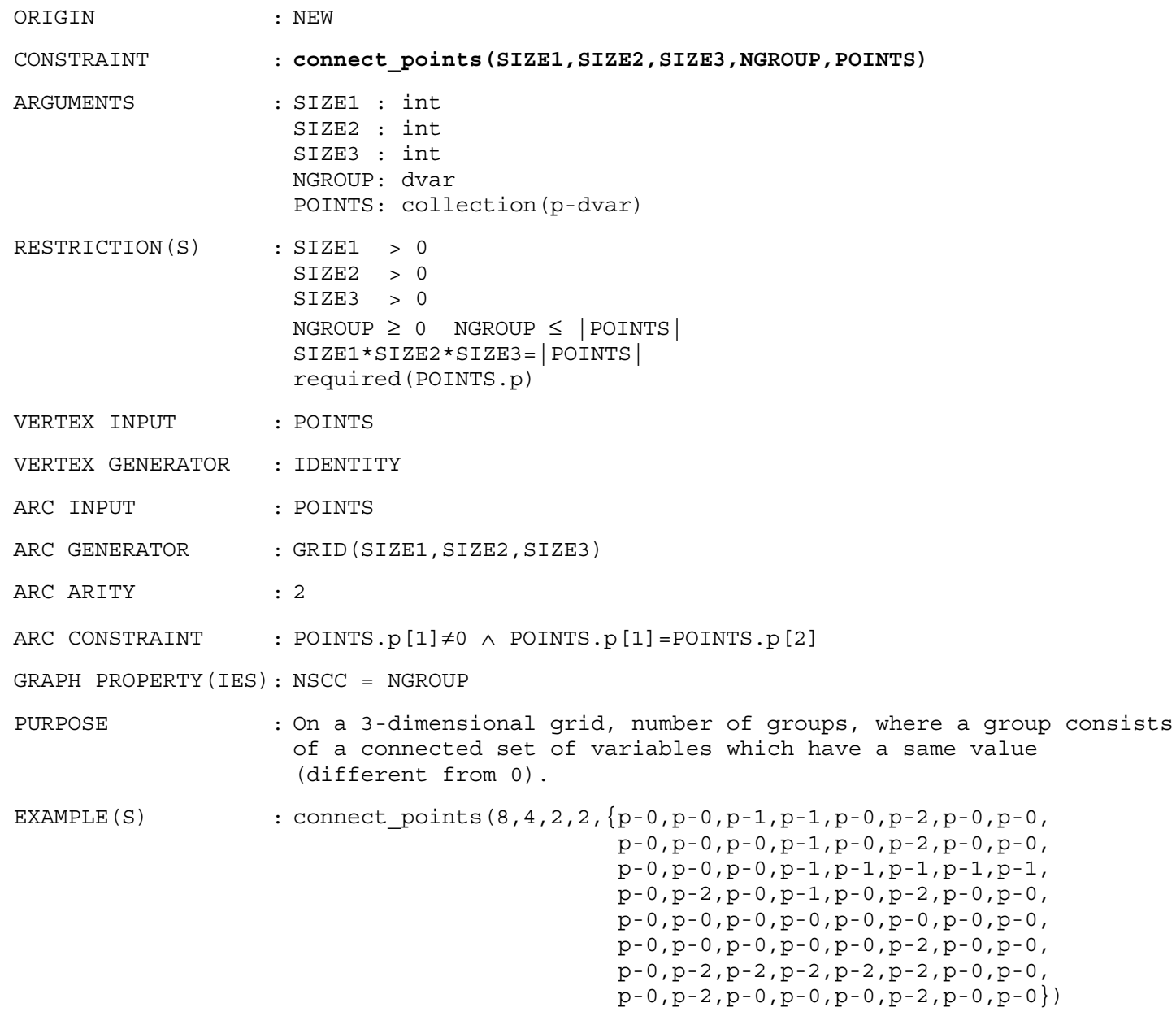

$\operatorname{EXAMPLE}(\mathrm{S})$

: connect_points $(8,4,2,2,\{\mathrm{p}-0, \mathrm{p}-0, \mathrm{p}-1, \mathrm{p}-1, \mathrm{p}-0, \mathrm{p}-2, \mathrm{p}-0, \mathrm{p}-0$, $\mathrm{p}-0, \mathrm{p}-0, \mathrm{p}-0, \mathrm{p}-1, \mathrm{p}-0, \mathrm{p}-2, \mathrm{p}-0, \mathrm{p}-0$, $\mathrm{p}-0, \mathrm{p}-0, \mathrm{p}-0, \mathrm{p}-1, \mathrm{p}-1, \mathrm{p}-1, \mathrm{p}-1, \mathrm{p}-1$, $\mathrm{p}-0, \mathrm{p}-2, \mathrm{p}-0, \mathrm{p}-1, \mathrm{p}-0, \mathrm{p}-2, \mathrm{p}-0, \mathrm{p}-0$ $\mathrm{p}-0, \mathrm{p}-0, \mathrm{p}-0, \mathrm{p}-0, \mathrm{p}-0, \mathrm{p}-0, \mathrm{p}-0, \mathrm{p}-0$, $\mathrm{p}-0, \mathrm{p}-0, \mathrm{p}-0, \mathrm{p}-0, \mathrm{p}-0, \mathrm{p}-2, \mathrm{p}-0, \mathrm{p}-0$ $\mathrm{p}-0, \mathrm{p}-2, \mathrm{p}-2, \mathrm{p}-2, \mathrm{p}-2, \mathrm{p}-2, \mathrm{p}-0, \mathrm{p}-0$, $\mathrm{p}-0, \mathrm{p}-2, \mathrm{p}-0, \mathrm{p}-0, \mathrm{p}-0, \mathrm{p}-2, \mathrm{p}-0, \mathrm{p}-0\})$

The next figure gives the initial graph that is generated by the arc generator.

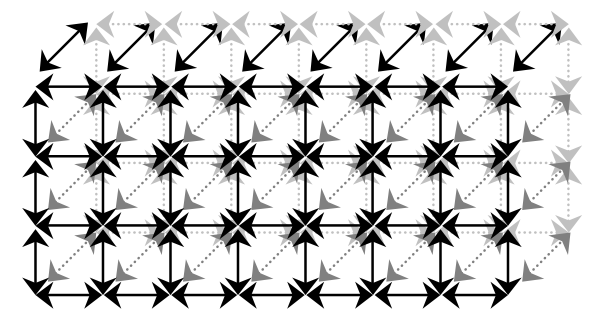

Figure 50. Graph generated by $\operatorname{grid}(8,4,2)$.

The next figure corresponds to the solution where we describe separately each layer of the grid. We have 2 groups, one for the variables that take value 1 , and one for the variables that take value 2 .

\begin{tabular}{|l|l|l|l|l|l|l|l|}
\hline 0 & 0 & $\mathbf{1}$ & $\mathbf{1}$ & 0 & $\mathbf{2}$ & 0 & 0 \\
\hline 0 & 0 & 0 & $\mathbf{1}$ & 0 & $\mathbf{2}$ & 0 & 0 \\
\hline 0 & 0 & 0 & $\mathbf{1}$ & $\mathbf{1}$ & $\mathbf{1}$ & $\mathbf{1}$ & $\mathbf{1}$ \\
\hline 0 & $\mathbf{2}$ & 0 & $\mathbf{1}$ & 0 & $\mathbf{2}$ & 0 & 0 \\
\hline
\end{tabular}

\begin{tabular}{|c|c|c|c|c|c|c|c|}
\hline 0 & 0 & 0 & 0 & 0 & 0 & 0 & 0 \\
\hline 0 & 0 & 0 & 0 & 0 & 2 & 0 & 0 \\
\hline 0 & 2 & 2 & 2 & 2 & 2 & 0 & 0 \\
\hline 0 & 2 & 0 & 0 & 0 & 2 & 0 & 0 \\
\hline
\end{tabular}

Figure 51. The 2 layers of the solution. 


\section{Algorithmic issues}

At a first glance, the global constraint description that we presented in the previous section sounds nice since it allows expressing in a very compact form a wide range of existing and new global constraints. However, a practical question that rises out immediately is how can one ever hope to implement such a huge number of global constraints. In this section we try to answer partially this question. However, one should keep in mind that these are very preliminary comments, since algorithmic issues were not the main topic of this report.

\subsection{Generic programs for performing basic tasks}

One of the advantages of our explicit and general description of global constraints is that one can come out with generic programs, which can be applied on any constraint that can be described with our formalism, in order to perform the following tasks:

- Parse and check the parameters of the global constraints,

- Generate automatically test cases for the global constraints,

- Generate automatically tests to capture missing propagation,

- Check if a global constraint holds for a given instantiated solution.

If we consider one single constraint, the previous tasks are quite obvious. However if we take a big number of global constraints then these tasks turns out to require too much energy in order to be done in a systematic way. If we want to allow users to define their own new global constraints then the first and last tasks are also a prerequisite. We now explain how we can come out with a generic checker for all global constraints:

- For each elementary constraint introduced in section 2.2.1., we have to supply an algorithm that evaluates if the constraint holds or not for a given instantiation of the variables of the elementary constraint.

- For each graph characteristic we have introduced in section 2.4., we have to provide a graph algorithm that takes a graph and that evaluates the value of the given characteristic. It would be more efficient to integrate directly within the previous algorithms the fact that we want to check that a particular characteristic is greater, equal or less than a given integer. Let us illustrate this point on a small example. Assume you want to check that the number of strongly connected component of a graph is less than 3. It would be better to integrate this test within the computation of the number of strongly connected component in order to return a failure as soon as we reach 3 components.

Now the structure of a general global constraint checker would be as following.

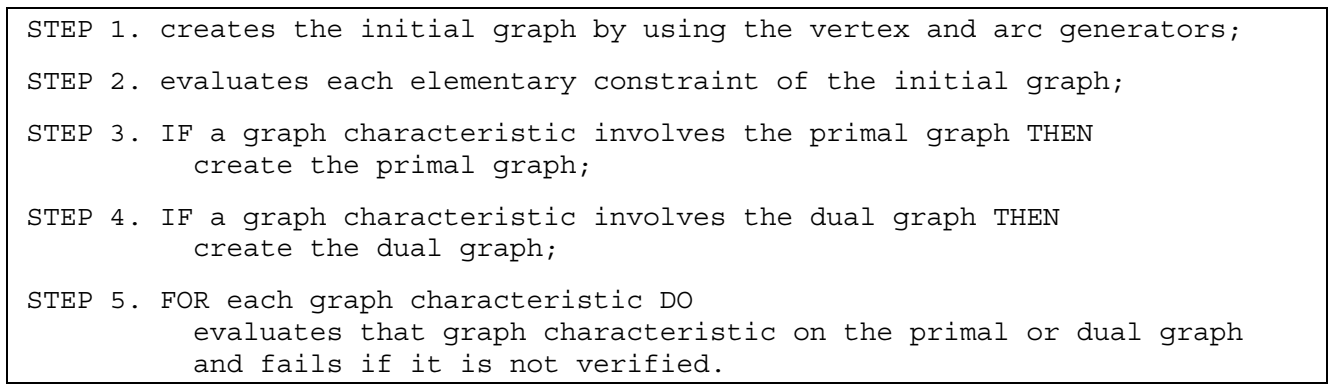

Algorithm 1. Generic checker for any global constraint.

Note that this generic checker could be refined according to the properties of the arcs generator and of the elementary constraints.

\subsection{Searching for general relations that links different graph characteristics}

In the list of examples of possible global constraints one can notice that quite often for a given global constraint, we put restrictions on different graph characteristics. Since these different characteristics are somehow strongly related we should systematically search these relations according to different additional hypothesis. Let us take a concrete example. If we consider the following graph characteristics of a graph $G$ that we introduce in section 2.4.:

- NVERTEX:
- NARC:
- NSCC:
- MIN_NSCC:
- MAX_NSCC:

cardinality of the set $V(G)$, cardinality of the set $E(G)$, number of strongly connected component of graph $G$, number of vertices of the smallest strongly connected component of graph $G$, number of vertices of the largest strongly connected component of graph $G$.

In a first step, we should look for the relations between theses 5 quantities that hold for any graph. In a second step we should try to refine the previous relations according the specific regular structure we consider (i.e. clique, line, chain, grid, ...), and according to the properties of the elementary constraints (i.e. symmetric, anti-symmetric, ...) 
associated to the arcs. By proceeding this way we would come up with a set of formulas that can be applied on any global constraints as soon as some properties hold on the arcs generator and on the elementary constraint associated to each arc. These formulas would be used in order to perform propagation between variables that are associated to different graph characteristics.

\subsection{Creation of propagation algorithms specific to a given family of global constraints}

Instead of designing one propagation algorithm for each global constraint, we should first try to regroup the different global constraint in families, each family sharing some common characteristics. The propagation algorithms should be designed for each family of constraints. The following table gives a preliminary attempt of classification of some of the constraints we encountered so far.

\begin{tabular}{|c|c|c|c|c|}
\hline Family & Common & characteristics & $\begin{array}{l}\text { Arcs } \\
\text { properties }\end{array}$ & Examples \\
\hline Order & $\begin{array}{l}\text { Vertex } \\
\text { Arc } \\
\text { Arc } \\
\text { Graph }\end{array}$ & $\begin{array}{l}\text { generator: IDENTITY } \\
\text { generator: CLIQUE } \\
\text { arity : } \\
\text { property }: \text { ORDER }\end{array}$ & $\begin{array}{l}\text { Anti- } \\
\text { symmetric }\end{array}$ & $\begin{array}{l}\text { Minimum } \\
\text { Maximum } \\
\text { Minimum modulo } \\
\text { Min_n } \\
\text { Max_n }\end{array}$ \\
\hline CardPath & $\begin{array}{l}\text { Vertex } \\
\text { Arc } \\
\text { Arc } \\
\text { Graph }\end{array}$ & $\begin{array}{l}\text { generator: IDENTITY } \\
\text { generator: PATH } \\
\text { arity }: \\
\text { property }: \text { NARC }\end{array}$ & & $\begin{array}{l}\text { Among } \\
\text { Count } \\
\text { Change } \\
\text { Cyclic_change } \\
\text { Cyclic_change_joker } \\
\text { Smooth } \\
\text { Change_partition } \\
\text { Among_seq } \\
\text { Sliding_sum } \\
\text { Relaxed_sliding sum }\end{array}$ \\
\hline Alldifferent & $\begin{array}{l}\text { Vertex } \\
\text { Arc } \\
\text { Arc } \\
\text { Graph }\end{array}$ & $\begin{array}{l}\text { generator: } \\
\text { generator: } \\
\text { arity } \quad: 2 \\
\text { property }: \text { MAX_NSCC } \leq 1\end{array}$ & $\begin{array}{l}\text { Symmetric } \\
\text { Transitive }\end{array}$ & $\begin{array}{l}\text { Alldifferent } \\
\text { Alldifferent_except_0 } \\
\text { Golomb }\end{array}$ \\
\hline Nequivalence & $\begin{array}{l}\text { Vertex } \\
\text { Arc } \\
\text { Arc } \\
\text { Graph }\end{array}$ & $\begin{array}{l}\text { generator: IDENTITY } \\
\text { generator: CLIQUE } \\
\text { arity : } \\
\text { property }: \text { NSCC }\end{array}$ & $\begin{array}{l}\text { Reflexive } \\
\text { Symmetric } \\
\text { Transitive }\end{array}$ & $\begin{array}{l}\text { Nvalue } \\
\text { Nequivalence } \\
\text { Nclass }\end{array}$ \\
\hline Ngroup (c) & $\begin{array}{l}\text { Vertex } \\
\text { Arc } \\
\text { Arc } \\
\text { Graph }\end{array}$ & $\begin{array}{ll}\text { generator: } & \text { IDENTITY } \\
\text { generator: } & - \\
\text { arity }: & 2 \\
\text { property }: & \text { NSCC }\end{array}$ & $\begin{array}{l}\text { Reflexive } \\
\text { Symmetric }\end{array}$ & $\begin{array}{l}\text { Connect_points (grid) } \\
\text { Connected (clique) }\end{array}$ \\
\hline
\end{tabular}

Table 4. Different families of constraints and their characteristics.

\section{Uncovered topics}

This section discusses various uncovered issues that were not addressed in sections 2 and 3 . We first present some existing and new global constraints that do not fit in the previous framework. We then refer to some existing combination of global constraints that were already addressed in the literature. Finally, we mention the problems of preemptive scheduling and of optimisation criteria.

\subsection{Exotic global constraints}

\subsubsection{The sort constraint of arity 3}

An already existing global constraint that does not fit in our framework is the sort constraint that was introduced in [ZHOU 97]. This constraint has the form $\operatorname{sort}\left(x_{1}, \ldots, x_{n}, s_{1}, \ldots, s_{n}, p_{1}, \ldots, p_{n}\right)$ where $\left(s_{1}, \ldots, s_{n}\right)$ is a $n$-tuple obtained by sorting in increasing order the domain variables of the $n$-tuple $\left(x_{1}, . ., x_{n}\right)$ and where $\left(p_{1}, . ., p_{n}\right)$ is the permutation that relates $\left(s_{1}, \ldots, s_{n}\right)$ to $\left(x_{1}, \ldots, x_{n}\right)$. The main reason that is does not fit is that the sort constraint has 3 parameters and that all the nodes of the graph of our global constraints are only build from one or 2 collections of objects.

\subsubsection{The case constraint}

The element constraint was originally introduced in [VAN HENTENRYCK \& CARILLON 88]. It has the form element(Index,List,Value), where Index and Value are domain variables and List is a list of integers. The constraint imposes that Value is equal to the Index th element of the list List. It was extended in different ways [COSYTEC 97], [CARLSSON, OTTOSSON \& CARLSON 97] in order to provide more compact forms of the list or in order to handle the 2-dimensional case. From our experience, we also notice that quite often, one states a set of element constraints with exactly the same list of values. In this last case, it appears also that sometimes all the 
Index variables have to take distinct values. From all the facts exposed before, we propose to generalize the element constraint and to introduce the case constraint

$$
\text { case(Tuples, Tree) }
$$

where Tuples corresponds to a list of tuples of domain variables, all tuples having the same arity. Tree corresponds to a tree, each node of the tree referring to a restriction of the variable associated to a specific position within the tuples of domain variables and to a list of binary constraints. The restriction is defined by a given set of nonnegative values and corresponds to the fact that the variable takes a value in the corresponding list of values. Each variable that occurs in a binary constraint refer also to a specific position within the tuples. In order to be valid, a tree has to verify the following properties:

(1) On each path from a given leaf of the tree to the root of the tree a variable can occurs at most once,

(2) At a given level of the tree the set of restrictions are pairwise incompatible.

The case constraint holds if every tuple can be assigned to a leaf of the tree in such a way that all restrictions and binary constraints that occur on the path between the root of the tree and the given leaf hold. These restrictions and constraints are applied on the variables of the tuple. Note that, since at any level of the tree the restrictions are pairwise incompatible, a tuple can only be assigned to a unique leaf of the tree.

Finally we propose to associate to each leaf of the tree one non-negative integer that corresponds to the maximum number of tuples that can be assigned to that specific leaf. We now give several examples of specific constraints that can be build using the case constraint. For each example we have chosen to give an ad-hoc syntax that is tailored to the specific example we are dealing with.

The first example shows how to represent a 2-dimensional element constraint using the case constraint. The constraint element $(I, J$, Table,$V)$ holds if $V$ is equal to Table $[I, J]$. On the first half of the next figure we show a matrix and the corresponding tree that should be generated for the case constraint. On the second half, we show how to represent a calendar constraint that links the machine $M$ to which a task is assigned, the real origin $R$ when the task starts and the virtual origin $V$ which corresponds to the fact that we have remove all unavailability periods on the different machines. This constraint is quite useful for some scheduling problems where one has resources on which unavailability periods are predefined and where tasks can be interrupted by these unavailabilities. In this kind of problem one associates a real and a virtual origin to each task. All the resource constraints are stated on the virtual origins, while all the temporal constraints are stated between the real origins. The calendar constraint makes the link between the virtual and the real origin according to the resource that is selected for a given task.

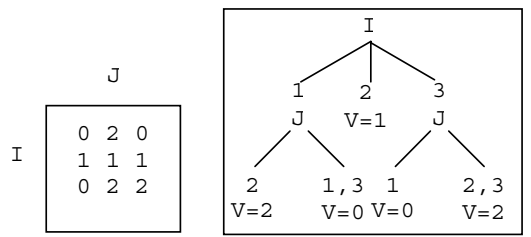

element (I, J, $[[0,2,0],[1,1,1],[0,2,2]], \mathrm{V})$

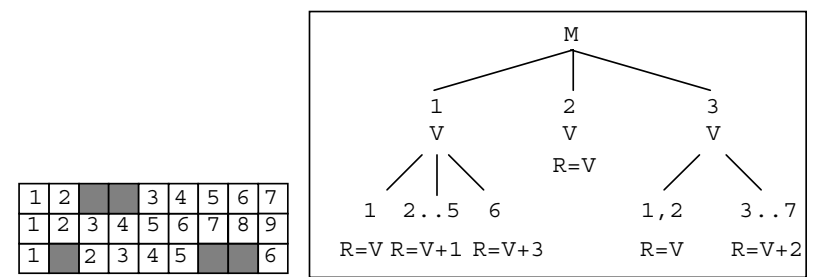

Calendar $([M, R, V],[1-[], 2-[1-1,7-8], 3-[3-4])$

Figure 52. 2-dimensional element constraint and calendar constraint

We now give an informal description of the principle of the 2 basic algorithms that should be used in order to handle the case constraint. The first algorithm enforces each tuple to be assigned on a leaf of the tree. For this purpose it scans the tree from the root to the leaves and performs propagation according to the restrictions and to the constraints encountered so far on the current path. When it reaches a leaf of the tree it records the current state of the variables of the tuple. It then makes a generalisation of all the states by computing the union of all these states recorded at the leaves of the tree. Finally it enforces the variables of the tuple it currently considers to belong to the previous sets. The second algorithm handles the capacity constraint associated to each leaf of the tree. For this purpose we create a bipartite graph where the first class of nodes corresponds to the tuples and the second class of nodes corresponds to the leaves of the tree where each leaf is implicitly duplicated according to its maximum capacity. An arc is created between a given tuple and a given leaf if the tuple can be assigned to that leaf (i.e. if all the restrictions and the constraints that are on the path that starts at the root of the tree and ends to the leaf do not lead to a contradiction when they are applied to the variables of the tuple). A bipartite maximum cardinality matching algorithm [ALT, BLUM, MEHLHORN \& PAUL 91] and a pruning algorithm [COSTA 94] are then applied. The result of the pruning algorithm consist of 2 actions. The first action corresponds to enforce a given arc by stating all the constraints and restrictions that are on the path that lead to the leaf associated to the arc. The second action remove one arc; this is performed by enforcing that at least one restriction or one constraint associated to the leaf fails. 


\subsubsection{The periodic constraint}

The periodic constraint is a new global constraint that is useful for periodic timetabling problems where one does not know in advance the length of the period of the schedule. It has the form periodic $\left(P,\left[X_{1}, . ., X_{n}\right], C t r\right)$, where $P$ and $\left[X_{1}, . ., X_{n}\right]$ are domain variables, and $C t r$ is a binary constraint. In typical applications one would like to try to minimize the value of variable $P$. The periodic constraint holds if $P$ is the smallest value such that for all integer values $i$ in interval $[1, n-P+1]$ the binary constraint $X_{i} C \operatorname{tr} X_{n-P+1}$ holds. Since the period $P$ is not initially fixed we cannot generate an initial graph and this is why this constraint does not fit in our classification.

\subsubsection{The inflexion constraint}

The inflexion constraint is a new global constraint that is useful for constraining the number of "peaks" or "valleys" of a sequence of domain variables. It has the form inflexion $\left(I,\left[X_{1}, \ldots, X_{n}\right], C t r, E q\right)$, where $I$ and $\left[X_{1}, \ldots, X_{n}\right]$ are domain variables, and $C t r$ and $E q$ are binary constraints such that $E q$ is an equivalence relation. The inflexion constraint holds if $I$ is the number of times that the following conjunctions of constraints hold:

$$
\left\{\begin{array}{c}
X_{i} \operatorname{Ctr} X_{i+1} \wedge X_{i} \neg E q X_{i+1} \\
X_{i+1} E q X_{i+2} \wedge \ldots \wedge X_{j-2} E q X_{j-1} \quad(1 \leq i, i+2 \leq j, j \leq n) . \\
X_{j-1} \neg E q X_{j} \wedge X_{j-1} \neg C t r X_{j}
\end{array}\right.
$$

When $C t r$ is equal to the constraint < (respectively >) and when $E q$ is the equality constraint then $I$ corresponds to the number of peaks (respectively valleys) of the sequence of variables $\left[X_{1}, . ., X_{n}\right]$. Because of the equivalence relation, the previous conjunction of constraints does not correspond to a ternary constraint as one could first think. Since the arity of our elementary constraint is not fixed, the inflexion constraint cannot be put into our framework. However, it would fit if we were introducing a new field that specifies how to merge adjacent nodes of our primal graph.

\subsection{Combination of global constraints}

One can find in the existing constraint field, a few papers that present some global constraints that combine different constraints in order to get a stronger propagation. This is for example the case for [BELDICEANU 90] where different alldifferent constraints are group together with several disequality constraints on pair of variables and in [BELDICEANU, BOURREAU, RIVREAU \& SIMONIS 96] where several cumulative constraints were combined with a precedence graph. This is also the case in [RÉGIN \& RUEHER 99] where a sum constraint is combined with binary inequalities. Since all the previous examples combine 2 different types of constraints we cannot express this in our framework. However one could think to extend it in order to allow combining 2 global constraints if their graphs have the same set of vertices.

\subsection{Preemptive scheduling}

In the catalog of global constraints we have come up with a big variety of resource scheduling constraints. However, since we do not have sets but just domain variables, our framework does not allows to express preemptive scheduling [BAPTISTE \& LEPAPE 97].

\subsection{Optimisation criteria}

For problems which deal with optimisation, it would be quite useful to introduce an optimisation field [GRAHAM, LAWLER, LENSTRA \& RINNOOY KAN 79, page 290], [HERROELEN, DEMEULEMEESTER \& DE REYCK 98, pages 14-16] which allows to express that a specific variable of a global constraint is equal to the minimum, maximum or sum of some attribute of the nodes of our primal graph. This would allow trying to get bounds for that cost variable according to the specificities of the global constraint and to propagate backward from the cost variable to the variables of the global constraint. One can note that this was already done on a case-by-case basis for some existing global constraints like:

- The maximum end of the tasks of the cumulative constraint [AGGOUN \& BELDICEANU 93],

- The maximum end in a specific dimension for the objects of the diffn constraint [BELDICEANU \& CONTEJEAN 94],

- The total cost of the assignment problem [FOCCACI, LODI \& MILANO 99].

However we did not yet introduce an optimisation field since we don't see how to come up with general algorithms for performing in an efficient way the corresponding propagation. 


\section{Communication between global constraints}

In most ${ }^{43}$ of the current constraint systems, constraints communicate through variables that are present in more than one constraint. This has been advocated as a strong point of the constraint approach over classical algorithms since one can add new constraint without considering the existing constraints. However this has also a severe drawback: sets of constraints are not handled in a global way, which sometimes lead to poor propagation [BOURGINE 86]. Quite often, if someone is looking carefully to the domain of the variables, it happen that obvious propagation steps that do not require any sophisticated algorithms are missing, due to the lack of communication between 2 given constraints. To get around this problem one way is to use shaving in order to enforce propagation, one other way is to regroup different constraints into a single global constraint in order that this new global constraint has all the relevant information to perform as much propagation as possible. However this has the severe drawback to create arbitrary combination of basic constraints. In order to partially avoid this problem we propose to add the following basic primitive into the constraint engine: ask_what_if(Info, Expression, Restrictions). This primitive allows asking for the minimum (Info=min) or maximum (Info=max) value of a given expression that contains domain variables according to a list of restrictions. Each restriction corresponds to an elementary constraint that involves only one single domain variable. This elementary constraint can take one of the following form: $X=v a l, X \neq v a l, X>v a l, X \leq v a l, X<v a l$ and $X \geq v$ al where $X$ is a domain variable and val a non negative integer. The ask_what_if primitive should be systematically used in the following cases:

- Each time that in an algorithm associated to a constraint you compute the minimum or maximum value of a variable according to a restriction you should make a query to the outside world in order to try to get a better estimation of the minimum or maximum value of that variable.

- Each time that in an algorithm associated to a constraint you compute the minimum or maximum value of an expression you should also make a query to the outside world for the same reason than above. In this case, it may be worthwhile to make a query even if no restriction applies.

- Each time that in an algorithm associated to a constraint you do some pruning only if some conditions that involve domain variables have to hold you should make queries to the outside world in order to get sharper bound for the variables in order to maximize the chance that the test conditions for the pruning are evaluated to true.

We now give 3 examples where the proposed mechanism would perform more propagation than the one obtained by considering the constraints independently.

\section{Example 1 interaction of non-linear arithmetic constraints}

Let us assume we want to handle the following arithmetic constraints:

(1) Low $\leq \sum_{i=1}^{n} X_{i} \cdot Y_{i} \leq U p$

(2) $\forall i \in 1 . . n:$ Low $_{i} \leq X_{i} \cdot Y_{i} \leq U p_{i}$

When we implement the algorithm that handle the first constraint we evaluate the minimum and maximum value of the $\mathrm{i}^{\text {th }} \mathrm{X}$ variable by isolating the term that contains the corresponding variable and obtain the following formulas:

$$
\begin{aligned}
& \text { if } \max \left(Y_{i}\right)>0 \text { then } \min \left(X_{i}\right) \geq\left[\frac{\text { Low }-\sum_{j=1, j \neq i}^{n} \max \left(X_{j} \cdot Y_{j}\right)}{\max \left(Y_{i}\right)}\right], \\
& \text { if } \min \left(Y_{i}\right)>0 \text { then } \max \left(X_{i}\right) \leq\left\lceil\frac{U p-\sum_{j=1, j \neq i}^{n} \min \left(X_{j} \cdot Y_{j}\right)}{\min \left(Y_{i}\right)}\right]
\end{aligned}
$$

In the previous formulas we have to evaluate the quantities $\min \left(X_{j} \cdot Y_{j}\right)$ and $\max \left(X_{j} \cdot Y_{j}\right)$. The obvious way would be to use following 2 identities:

$$
\begin{aligned}
& \min \left(X_{j} \cdot Y_{j}\right) \geq \min \left(X_{j}\right) \cdot \min \left(Y_{j}\right), \\
& \max \left(X_{j} \cdot Y_{j}\right) \leq \max \left(X_{j}\right) \cdot \max \left(Y_{j}\right) .
\end{aligned}
$$

\footnotetext{
${ }^{43}$ This is not true for Alice [LAURIÈRE 76 pages 53-61] where specific class of constraints were communicating in an ad-hoc way in order to derive stronger constraints. This was for example the case for the arithmetic constraints.
} 
However if we have the second type of constraints, the previous identities could underestimate the quantities we want to evaluate and thus lead to less propagation. In order to avoid this problem we should replace the 2 identities by the 2 following queries:

$$
\begin{aligned}
& \text { ask_what_if(min, } \left.X_{j} \cdot Y_{j},[]\right) \text {, } \\
& \text { ask_what_if(max, } \left.X_{j} \cdot Y_{j},[]\right) .
\end{aligned}
$$

\section{Example 2 interaction of cumulative and element constraints}

Let us assume we have to schedule a set of tasks under a given resource limitation Limit. Suppose that the height of each task depends of the starting time of the task. In this problem, the resource constraint can be modeled by a cumulative constraint while the fact that the height of a task depends of the origin can be represented by an element constraint. Assume that we handle the cumulative constraint by computing the cumulated profile, which consist of the different obligatory part [LAHRICHI 82], [LAHRICHI \& GONDRAN 84], [CASEAU \& LABURTHE 96b] of the different tasks. A basic pruning operation has to be performed each time the cumulated profile increase on a given interval inf..sup of height $h$. Usually for a task of origin $\mathrm{O}$, duration $\mathrm{D}$ and height $\mathrm{R}$ that do not have any obligatory part already registered on the interval inf..sup, this pruning can be implemented by the following peace of code:

$$
\text { if } \min (\mathrm{R})>\max (\text { Limit) }-\mathrm{h} \text { then remove interval inf-min(D) }+1 \ldots \text { sup from } \mathrm{O}
$$

However, if the height of the task depends of the starting point of the task the test $\min (\mathrm{R})>\max ($ Limit $)$-h may not be evaluated to true because we underestimate the value of $\min (\mathrm{R})$. If the height of the task depends of the starting point of the task then we should also try to have a better estimation of the minimum height of the task. For these reasons the previous code should be rewrite in the following way:

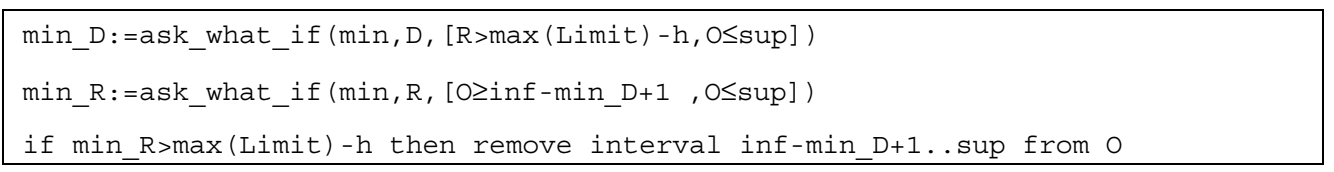

The reason of that is that when we remove an interval of values from a domain variable we implicitly make the assumption that the variable will take one of the values of that interval. This is why we should systematically revisit the conditions that we evaluate in order to check if we can prune or not.

\section{Example 3 interaction of cycle and element constraints}

Let us assume we use the cycle constraint [BELDICEANU \& CONTEJEAN 94] and the element constraint in order to solve a tour planning where one wants to impose a strong limitation of the maximum distance that is done by each vehicle. In order to model this problem we could use the following constraints:

$$
\begin{aligned}
& \operatorname{cycle}\left(\mathrm{Nb} \_c y c l e,\left[\mathrm{Next}_{1}, \ldots, \mathrm{Next}_{\mathrm{n}}\right],\left[\mathrm{Dist}_{1}, \ldots, \mathrm{Dist}_{\mathrm{n}}\right], 0, \mathrm{Max} \mathrm{Dist}\right) \\
& \text { element }\left(\mathrm{Next}_{1}, \text { Distance_table_from_location_1,Dist }{ }_{1}\right) \ldots \text { element }\left(\mathrm{Next}_{\mathrm{n}}, \text { Distance_table_from_location_n,Dist }{ }_{\mathrm{n}}\right)
\end{aligned}
$$

In these constraints, $\mathrm{Nb}$ _cycle corresponds to the number of tours that will be proceed, Next $\mathrm{i}_{\mathrm{i}}$ to the location which is located immediately after location $i$, and Dist ${ }_{i}$ to the distance between location i and location Next $\mathrm{i}_{\mathrm{i}}$.

Lets assume that we handle each path and it corresponding minimum length (the sum of the minimum distance variables associated to the nodes of the path). A path corresponds to a succession of fixed nodes and is denoted by its first fixed node and by the successor of its last fixed node. If we want to try to prune the successor variable according to the maximum distance constraint we have to check if the merging of 2 paths will produce a distance overflow or not. For this purpose we could write this line of code:

$$
\begin{aligned}
& \text { if minimum length of path } i+\text { minimum length of path } j>\text { MaxDist } \\
& \text { then remove start of path } j \text { from the successor variable of the end of path } i
\end{aligned}
$$

However the previous test may not be evaluated to true because we assume the fact that connecting path $i$ to path $j$ will cost us a distance which is equal to the minimum value of the distance variable associated to the last node of path i. In order to get a more accurate test we should count the real distance between the node that corresponds to the end of line $i$ and the node that corresponds to the start of line $\mathrm{j}$. Since this information is not directly available within the cycle constraint, we should rewrite the previous line of code in the following way in order to get the correct distance from the element constraint.

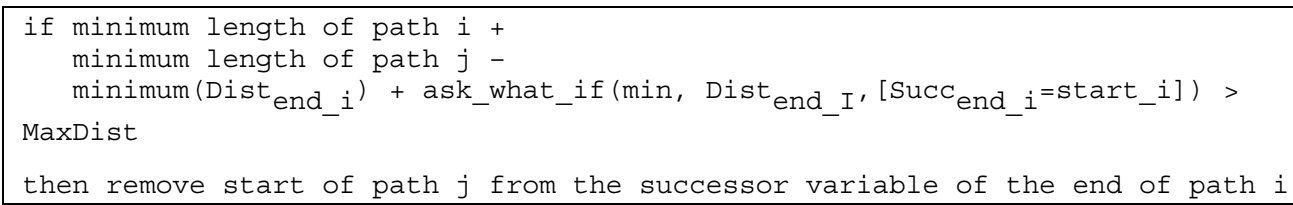




\section{Conclusion}

In this report we have introduced a classification of global constraints that allows to reconstruct and to generalize almost all existing global constraints and to generate new interesting global constraints. A first advantage of this classification scheme is that it is not domain specific: it regroups constraints from different areas such as scheduling, placement, logistics, geometry and timetabling. A second benefit of this classification is that, since it is related to the internal structure of the constraint, it gives also an indication of the algorithms that will be associated to the constraints. It enlights the strong relationship between some global constraints that use a same graph property and binary constraints with the same structural properties. This will allow regrouping apparently differing global constraints in families for which one can hope to come up with similar algorithms. These algorithms will have to reuse and adapt some of the existing work that was done on efficient data structures and on graph algorithms. Finally a last benefit of this unified view is that, it should facilitate to perform in a systematic way such tasks as designing visualisation interfaces, generating linear relaxations and expressing heuristics for global constraints.

\section{Acknowledgement}

I express thanks to Mats Carlsson, Per Kreuger and Helmut Simonis for their helpful comments on this report.

\section{Appendix}

We shortly recall the definition of the cumulative, the among, the diffn and the cycle constraints. The cumulative constraint was introduced in [AGGOUN \& BELDICEANU 93] in order to model scheduling problems where one has to deal with a resource of limited capacity. It has the following definition:

$$
\text { cumulative }\left(\left[O_{1}, \ldots, O_{n}\right],\left[D_{1}, . ., D_{n}\right],\left[R_{1}, . ., R_{n}\right], L\right),
$$

where $\left[O_{1}, \ldots, O_{n}\right],\left[D_{1}, . ., D_{n}\right]$ and $\left[R_{1}, \ldots, R_{n}\right]$ are nonempty lists of domain variables, and where $L$ is a non negative-integer. The constraint holds if the following condition is true:

$$
\forall i \in \mathrm{N} \sum_{j \mid O_{j} \leq i \leq O_{j}+D_{j}-1} R_{j} \leq L .
$$

From an interpretation point of view, the cumulative constraint matches the single resource-scheduling problem, where $O_{1}, \ldots, O_{n}$ correspond to the start of the tasks, $D_{1}, \ldots, D_{n}$ to the duration of the tasks, and $R_{1}, \ldots, R_{n}$ to the amount of resource used by each task. The cumulative constraint specifies that, at any instant $i$, the summation of the amount of resource of the tasks that overlap $i$, does not exceed the limit $L$.

The among constraint was introduced in [BELDICEANU \& CONTEJEAN 94] and can be seen a special case of the cardinality combinators [VAN HENTENRYCK \& DEVILLE 91]. It has the following definition:

$$
\operatorname{among}\left(N,\left[X_{1}, \ldots, X_{n}\right],\left[V_{1}, \ldots, V_{m}\right]\right) \text {, }
$$

where $N$ is a domain variable, $\left[X_{1}, \ldots, X_{n}\right]$ is a list of domain variables and $\left[V_{1}, \ldots, V_{m}\right]$ is a list of integers. The constraint holds if exactly $N$ variables among $\left[X_{1}, \ldots, X_{n}\right]$ take their value in the list of values $\left[V_{1}, \ldots, V_{m}\right]$.

The diffn constraint was introduced in [BELDICEANU \& CONTEJEAN 94] in order to handle multidimensional placement problems that occur in scheduling and placement problems and has the following definition:

$$
\operatorname{diffn}\left(\left[O_{11}, \ldots, O_{1 n}, L_{11}, \ldots, L_{1 n}\right], \ldots \llbracket\left[O_{m 1}, \ldots, O_{m n}, L_{m 1}, \ldots, L_{m n}\right] \rrbracket\right),
$$

where for all $i \in[1, m]$ and for all $j \in[1, n], O_{i j}$ and $L_{i j}$ are domain variables. The constraint holds if the following condition is true:

$$
\forall i \in[1, m], \forall j \in[1, n] j \neq i, \exists k \in[1, n] \mid O_{i k} \geq O_{j k}+L_{j k} \vee O_{j k} \geq O_{i k}+L_{i k} .
$$

From an interpretation point of view, this condition is that for each pair $i, j(i \neq j)$ of $n$-dimensional rectangle there exists at least one dimension $k$ where $i$ is after $j$ or $j$ is after $i$.

The cycle constraint was introduced in [BELDICEANU \& CONTEJEAN 94] in order to tackle vehicle routing problems. It has the following definition:

$$
\operatorname{cycle}\left(N,\left[S_{1}, \ldots, S_{m}\right]\right),
$$

where $N$ is a domain variable, and $\left[S_{1}, \ldots, S_{m}\right]$ is a nonempty list of domain variables. The constraint holds if the following conditions are both true:

$$
\begin{gathered}
\forall i \in[1, m]: 1 \leq S_{i} \leq m, \\
\forall i \in[1, m], \forall j \neq i \in[1, m]: S_{i} \neq S_{j},
\end{gathered}
$$


$\forall i \in[1, m]$, let $C_{i}$ be the set such that: $i \in C_{i}$, if $j \in C_{i}$ then $S_{j} \in C_{i}$; then we have exactly $N$ distincts sets.

\section{References}

[AGGOUN \& BELDICEANU 93]

A. Aggoun and N. Beldiceanu. Extending CHIP in order to solve complex scheduling and placement problems, Mathl. Comput. Modelling 17 (7), 57-73 (1993).

[ALCOM 97]

http://www.dis.uniroma1.it/ alcom-it/

[ALT, BLUM, MEHLHORN \& PAUL 91]

H. Alt, N. Blum, K. Mehlhorn and M. Paul. Computing a maximum cardinality matching in a bipartite graph in time $\mathbf{O}\left(n^{1.5} \sqrt{m / \log n}\right)$. Information Processing Letters 37, 237-240, (1991).

\section{[BAPTISTE \& LEPAPE 97]}

P. Baptiste and C. Le Pape. An Experimental Comparison of Constraint-Based Algorithms for the Preemptive JobShop Scheduling Problem. In Proceedings of the CP'97 workshop on Industrial Constraint-directed Scheduling, (1997).

[BELDICEANU 90]

N. Beldiceanu. An Example of Introduction of Global Constraints in CHIP: Application to Block Theory Problems. ECRC Technical Report TR-LP-49, (15 May 1990).

[BELDICEANU, BOURREAU, RIVREAU \& SIMONIS 96]

N. Beldiceanu, E. Bourreau, D. Rivreau and H. Simonis. Solving Resource-constrained Project Scheduling Problems with CHIP. In proc. of Fifth International Workshop on Project Management and Scheduling (PMS'96), Poznan, 35-38, (1996).

[BELDICEANU \& CONTEJEAN 94]

N. Beldiceanu and E. Contejean. Introducing global constraint in CHIP. Mathl. Comput. Modelling Vol. 20, No. 12, 97-123 (1994).

[BERGE 70]

C. Berge. Graphes. Dunod, 1970; Graphs, Second Revised Edition. North-Holland, New York (1985).

\section{[BERGE 87]}

C. Berge. Hypergraphes, Combinatoire des ensembles finis. Dunod, (1987).

\section{[BESSIÈRE 99]}

C. Bessière. Non-Binary Constraints. Principles and Practice of Constraint Programming. In Principles and Practice of Constraint Programming - CP'99, 5th International Conference, Alexandria, Virginia, USA, (October 11-14, 1999), Proceedings. Lecture Notes in Computer Science, Vol. 1713, Springer, (1999).

\section{[BOURGINE 86]}

P. Bourgine. Propagation et extraction en résolution de problèmes. Colloque Intelligence Artificielle, Strasbourg, 271-287, (1986).

\section{[BOURREAU 99]}

E. Bourreau. Traitement de contraintes sur les graphes en programmation par contraintes. $\mathrm{PhD}$ thesis of University Paris 13, (March 30, 1999).

\section{[CARLSSON, OTTOSSON \& CARLSON 97]}

M. Carlsson, G. Ottosson, B. Carlson. An Open-Ended Finite Domain Constraint Solver. Proc. Programming Languages: Implementations, Logics, and Programs, (1997).

[CASEAU \& LABURTHE 96a]

Y. Caseau and F. Laburthe. Introduction to the Claire Programming Language. LIENS Report 96-15, École Normale Supérieure, (1996).

[CASEAU \& LABURTHE 96b]

Y. Caseau and F. Laburthe. Cumulative Scheduling with Task Intervals, Proceedings of the Joint International Conference and Symposium on Logic Programming, MIT Press, (1996). 
[CHEMLA, DIAZ, KERLIRSIN \& MANCHON 94]

D. Chemla, D. Diaz, P. Kerlirzin, S. Manchon. Using CLP(FD) to Suport Ait Traffic Flow Management.

Workshop on Constraint Languages and their Use in Problem Modelling, ILPS, Ithaca, NY, (November 1994).

[GUERNALEC \& COLMERAUER 97]

N. Bleuzen Guernalec and A. Colmerauer. Narrowing a $2 n$-Block of Sortings in $O(n \log n)$. In Principles and Practice of Constraint Programming - CP97, Third International Conference, Linz, Austria, (October 29 -

November 1, 1997), Proceedings. Lecture Notes in Computer Science, 2-16, Vol. 1330, Springer, (1997).

[COSTA 94]

M-C. Costa. Persistency in maximum cardinality bipartite matchings. Operation Research Letters 15, 143-149, (1994).

[COSYTEC 97]

COSYTEC. CHIP 5.1 Documentation, Addendum. Chip Reference Manual, Volume 5, (1997).

[COUSIN 93]

X. Cousin. Application of Constraint Logic Programming on Timetable Problem. PhD thesis, IRISA, (June 2, 1993).

[DECHTER \& PEARL 87]

R. Dechter, J. Pearl. Network-Based Heuristics for Constraint-Satisfaction Problems. Artificial Intelligence 34, $1-38,(1987)$.

[DECHTER, MEIRI \& PEARL 91]

R. Dechter, I. Meiri and J. Pearl. Temporal constraint networks. Artificial Intelligence 49, 61-95, (1991).

[DINCBAS, VAN HENTENRYCK, SIMONIS, AGGOUN, GRAF \& BERTHIER 88]

M. Dincbas, P. Van Hentenryck, H. Simonis, A. Aggoun, T. Graf and F. Berthier. The constraint logic programming language CHIP, In Proc. Int. Conf. On Fifth Generation Computer Systems FGCS-88, Tokyo, 693-702, (1988).

[FOCCACI, LODI \& MILANO 99]

F. Focacci, A. Lodi and M. Milano. Cost-Based Domain Filtering. In Principles and Practice of Constraint Programming - CP'99, 5th International Conference, Alexandria, Virginia, USA, (October 11-14, 1999), Proceedings. Lecture Notes in Computer Science, Vol. 1713, Springer, 189-203, (1999).

[GAEDE, BRODSKY, GÜNTHER, SRIVASTAVA, VIANU \& WALLACE 97]

V. Gaede, A. Brodsky, O. Günther, D. Srivastava, V. Vianu and M. Wallace (Eds). Constraint Databases and Applications. Lecture Notes in Computer Science 1191. Second International Workshop on Constraint Database Systems, CDB'97, Delphi, Greece, (January 1997).

[GAREY \& JOHNSON 79]

M. R. Garey and D. S. Johnson. Computers and intractability. A Guide to the Theory of NP-Completeness. W.H.Freeman and Company, San Francisco, (1979).

[GENDREAU 99]

M. Gendreau. Constraint Programming and Operations Research: Comments from an Operations Researcher. CRT Pub. No 99-41, (10 pages), (October 1999).

[GONDRAN \& MINOUX 79]

M. Gondran and M. Minoux. Graphes et algorithmes. Eyrolles, (1979); Graphs and Algorithms, Second Revised Edition, New York, Wiley, (1984).

[GOLOMB 65]

S. W. Golomb. Polyominoes, Scribners, NY, 1965; second edition (Polyominoes: Puzzles, Patterns, Problems, and Packings) Princeton Univ. Press, (1994).

[GRAHAM, LAWLER, LENSTRA \& RINNOOY KAN 79]

R. L. Graham, E. L. Lawler, J. K. Lenstra and A. H. G. Rinnooy Kan. 'Optimization and approximation in deterministic sequencing and scheduling: a survey’, Ann. Discrete Math., 5, 287-326 (1979).

[HARIDI, VAN ROY, BRAND \& SCHULTE 98]

S. Haridi, P. Van Roy, P. Brand, and C. Schulte. Programming Languages for Distributed Applications. New Generation Computing, n3 v16, (1998), Omsha, Ltd. and Springer-Verlag. 
[HERROELEN, DEMEULEMEESTER \& DE REYCK 98]

W. Herroelen, E. Demeulemeester, and B. De Reyck. A Classification Scheme for Project Scheduling Problems. in: Weglarz J. (Ed.), Handbook on Recent advances in Project Scheduling, Kluwer Academic Publishers, (1998).

[ILOG 99]

ILOG Solver 4.4 User's manual. ILOG S.A., (1999).

\section{[LAHRICHI 82]}

A. Lahrichi. Scheduling: the Notions of Hump, Compulsory Parts and their Use in Cumulative Problems. In C. R. Acad. Sc. Paris, t. 294, 209-211, (February 8 1982).

[LAHRICHI \& GONDRAN 84]

A. Lahrichi and M. Gondran. Théorie des parties obligatoires et découpes à deux dimensions. Research report HI/4762-02 from EDF (Électricité de France), (23 pages), (January 1984).

[LAPORTE, ASEF-VAZIRI \& SRISKANDARAJAH 96]

G. Laporte, A. Asef-Vaziri and C. Sriskandarajah. Some Applications of the Generalized Travelling Salesman Problem. Journal of the Operational Research Society 47, 1461-1467, (1996).

\section{[LAURIÈRE 76]}

J-L. Laurière. Un langage et un programme pour énoncer et résoudre des problèmes combinatoires. Thèse de Doctorat d'État de l'Université Paris 6, (May 1976).

[LAURIÈRE 78]

J-L. Laurière. A Language and a Program for Stating and Solving Combinatorial Problems. Artificial Intelligence 10, 29-127, (1978).

[LAURIÈRE 96]

J-L. Laurière. Constraint propagation or automatic programming ? IBP-Laforia research report Number 19, (1996).

[LABBÉ, LAPORTE \& RODRÍGUEZ-MARTÍN 98]

M. Labbé, G. Laporte and I. Rodríguez-Martín. Path, tree and cycle location. In Fleet Management and Logistics, Kluwer Academic Publishers, 187-204, (1998).

[MARTELLO \& TOTH 90]

S. Martello and P. Toth. Knapsack problems. Algorithms and Computer Implementations. Wiley-Interscience Series in Discrete Mathematics and Optimization, (1990).

[McKEE \& McMORRIS 99]

T. A. McKee and F. R. McMorris. Topics in Intersection Graph Theory. Siam Monographs on Discrete Mathematics and Applications, (February 1999).

[MEHLHORN \& NÄHER 99]

K. Mehlhorn and S. Näher. LEDA A Platform for Combinatorial and Geometric Computing, Cambridge University Press, (1999).

[NENG-FA ZHOU 96]

Neng-Fa Zhou. Channel Routing with Constraint Logic Programming and Delay. In 9th International Conference on Industrial Applications of AI, Gordon and Breach Science Publishers, pp.217-231, (1996).

[PACHET \& ROY 99]

F. Pachet and P. Roy. Automatic Generation of Music Programs. In Principles and Practice of Constraint Programming - CP'99, 5th International Conference, Alexandria, Virginia, USA, (October 11-14, 1999), Proceedings. Lecture Notes in Computer Science, Vol. 1713, Springer, (1999).

[PESANT \& SORIANO 98]

G. Pesant and P. Soriano. An Optimal Strategy for the Constrained Cycle Cover Problem. CRT Pub. no 98-65, (14 pages), (December 1998).

\section{[PITRAT 93]}

J. Pitrat. Penser autrement l'informatique. Edition Hermès, (1993).

[RÉGIN 94]

J-C. Régin. A filtering algorithm for constraints of difference in CSP. In Proc. of the Twelfth National Conference on Artificial Intelligence (AAAI-94), 362-367, (1994). 
[RÉGIN 96]

J-C. Régin. Generalized Arc Consistency for Global Cardinality Constraint. In Proc. of the Fourteenth National

Conference on Artificial Intelligence (AAAI-96), (1996).

[RÉGIN 99]

J-C. Régin. The symmetric alldiff constraint. In Proceedings IJCAI'99, Stockholm, Sweden, 420-425, (1999).

[RÉGIN \& RUEHER 99]

J-C. Régin and M. Rueher. A global constraint combining a sum constraint and binary inequalities. In Sixteenth International Joint Conference on Artificial Intelligence (IJCAI 99), Stockholm, Workshop on Non Binary

Constraints, (August 2 1999).

[ROY, LIRET \& PACHET 99]

P. Roy, A. Liret and F. Pachet. A Framework for Constraint Satisfaction. Research Report LIP6 1999/001, (1999).

[SAMET 89]

H. Samet. The design and analysis of spatial data structures. Addison-Wesley, (1989).

[SARASWAT 87]

V. A. Saraswat. Concurrent Logic Programming Languages. PhD thesis, Carnegie-Mellon University, (1987).

[SEDGEWICK 88]

R. Sedgewick. Algorithms. Addison-Wesley, (1988).

[SEDGEWICK \& FLAJOLET 96]

R. Sedgewick and P. Flajolet. An introduction to the analysis of algorithms. Addison-Wesley, (1996).

[SHEARER 96]

J. B. Shearer. http://www.research.ibm.com/people/s/shearer/grule.html.

[SKIENA 90]

S. Skiena. Implementing Discrete Mathematics. Combinatoric and Graph Theory with Mathematica. AddisonWesley, (1990).

[SIMONIS 90]

H. Simonis. Channel Routing Seen as a Constraint Problem. ECRC Technical Report TR-LP-51, 8 (June 1990).

[SIMONIS 96]

H. Simonis. A Problem Classification Scheme for Finite Domain Constraint Solving. In Proc. Workshop on constraint applications, CP96, Boston, (August 1996).

[SIMONIS 98]

H. Simonis. Visual CHIP - A Visual Language for Defining Constraint Programs. CCL II Working Group Meeting Dagstuhl, Germany, (September 1998).

[SIMONIS, AGGOUN, BELDICEANU \& BOURREAU 00]

H. Simonis, A. Aggoun, N. Beldiceanu and E. Bourreau. Global Constraint Visualization. To appear in chapter 12 in the Discipl book, (2000).

[SMITH, STERGIOU \& WALSH 99]

B. M. Smith, K. Stergiou and T. Walsh. Modelling the Golomb Ruler Problem. In Sixteenth International Joint Conference on Artificial Intelligence (IJCAI 99), Stockholm, Workshop on Non Binary Constraints, (August 2 1999).

[SMOLKA 96]

G. Smolka. Constraints in Oz. In ACM Computing Surveys, Vol 28, No 4, (December 1996).

[VAN HENTENRYCK \& CARILLON 88]

P. Van Hentenryck and J-P. Carillon. Generality versus Specificity: an Experience with AI and OR Techniques. In American Association for Artificial Intelligence (AAAI-88), St. Paul, Mi, (August 1988).

[VAN HENTENRYCK 89]

P. Van Hentenryck. Constraint Satisfaction in Logic Programming. The MIT Press, (1989).

[VAN HENTENRYCK \& DEVILLE 91]

P. Van Hentenryck and Y. Deville. The Cardinality Operator: A New Logical Connective for Constraint Logic Programming. ICLP 1991, 745-759, (1991). 
[VAN HENTENRYCK 99]

P. Van Hentenryck. The OPL Optimization Programming Language. The MIT Press, (1999).

[ZHOU 97]

J. Zhou. A permutation-based approach for solving the job-shop problem. Constraints, 2(2), 185-213, (1997). 
Index of the global constraints discussed

alldifferent, 35

alldifferent_except_0, 35

alldifferent_same_value, 62

among, 27

among_seq, 40

assign_and_count, 33

assign_and_nvalue, 34

balance, 28

bin_packing, 91

binary_tree, 75

calendar, 109

case, 108

change, 43

change_continuity, 53

change_partition, 47

circuit_cluster, 70

coloured_cumulative, 83

coloured_cumulatives, 89

common, 30

connect_points, 106

connected, 92

count, 27

crossing, 102

cumulative, 81

cumulative_2d, 104

cumulatives, 87

cycle, 67

cycle_card_on_path, 72

cycle_cover, 77

cycle_or_accessibility, 79

cyclic_change, 44

cyclic_change_joker, 45

cyclic_cumulative, 85

derangement, 64

diffn, 94

distance_change, 61

distance_less, 60 element, 108

golomb, 36

graph_crossing, 100

group, 49

group_skip_isolated_item, 51

inflexion, 110

interval_and_count, 58

interval_and_sum, 57

inverse, 68

longest_changes, 48

map, 76

max_n, 26

max_nvalue, 29

maximum, 23

maximum_modulo, 24

min_n, 25

min_nvalue, 29

minimum, 23

minimum_modulo, 24

nclass, 39

nequivalence, 38

notallequal, 37

nvalue, 37

orchad, 103

periodic, 110

place_in_pyramid, 96

polyomino, 98

relaxed_sliding_sum, 42

same, 31

sliding_card_skip0, 55

sliding_sum, 41

smooth, 46

sort, 108

symmetric_alldiff, 69

temporal_path, 65

tree, 74

used_by, 32 